Aus der Klinik für Psychiatrie und Psychotherapie

(Prof. Dr. med. J. Wiltfang)

der Medizinischen Fakultät der Universität Göttingen

\title{
Quantitative analysis of neuropathological alterations in two transgenic mouse models of Alzheimer's disease
}

\author{
INAUGURAL - DISSERTATION \\ zur Erlangung des Doktorgrades \\ der Medizinischen Fakultät der \\ Georg-August-Universität zu Göttingen
}

vorgelegt von

\section{Anastasiia Kurdakova \\ aus Kiew}

Göttingen 2016 
Dekan:

Referent/in:

Ko-Referent/in:
Prof. Dr. rer. nat. H. K. Kroemer

PD. Dr. rer. nat. O. Wirths

Prof. Dr. Inga Zerr

Tag der mündlichen Prüfung: 23.11.2016 


\section{Affidavit:}

I hereby declare that my doctoral thesis entitled "Quantitative analysis of neuropathological alterations in two transgenic mouse models of Alzheimer's disease" has been written independently with no other sources and aids than quoted.

Anastasiia Kurdakova

Göttingen, 04.05.2016 
Parts of this thesis have been published:

\section{Original articles:}

Richard BC*, Kurdakova A*, Baches S, Bayer TA, Weggen S, Wirths O (2015): Gene Dosage Dependent Aggravation of the Neurological Phenotype in the 5XFAD Mouse Model of Alzheimer's Disease. J Alzheimers Dis JAD $\underline{45}, 1223-1236$

*These authors contributed equally to this work.

Hüttenrauch M, Brauß A, Kurdakova A, Borgers H, Klinker F, Liebetanz D, Salinas-Riester G, Wiltfang J, Klafki H, Wirths O (2016): Physical activity delays hippocampal neurodegeneration and rescues memory deficits in an Alzheimer disease mouse model. Transl Psychiatry $\underline{6}$ e e800; doi: 10.1038/tp.2016.65

\section{Abstract:}

Wirths O, Brauß A, Kurdakova A, Hüttenrauch M, Bayer TA (2015): Physical activity ameliorates neuron loss and memory deficits in $\mathrm{Tg} 4-42$ mice. Poster presentation, The International Conference on Alzheimer's and Parkinson's Diseases. Neurodegener Dis 15(suppl 1): 352-1969, 1022 


\section{TABLE OF CONTENTS}

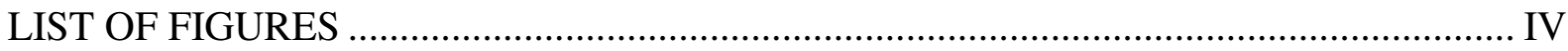

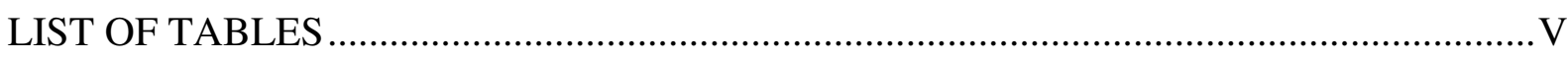

LIST OF ABBREVIATIONS...................................................................................... VI

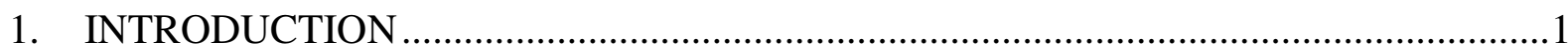

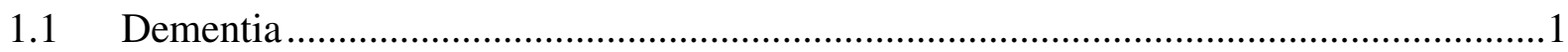

1.1.1 Epidemiology of dementia .....................................................................................

1.1.2 The topicality of a problem of dementia and the importance of the ongoing research in this field ....................................................................................................

$1.2 \quad$ Alzheimer's disease: history and epidemiology ……………………………………....

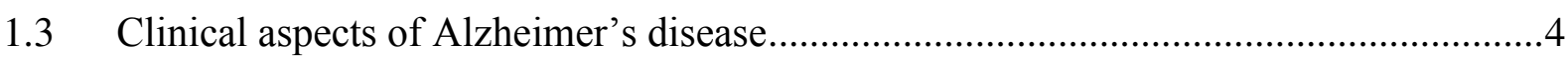

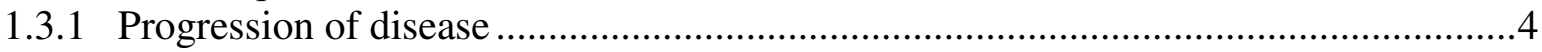

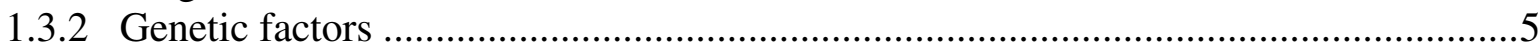

1.3.3 Risk factors for Alzheimer's disease........................................................................

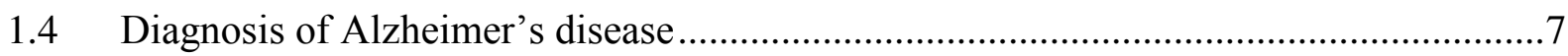

$1.5 \quad$ Neuropathological hallmarks of Alzheimer's disease ...................................................

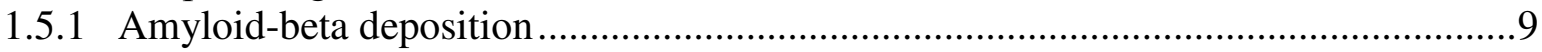

1.5.2 Neurofibrillary tangles ..................................................................................11

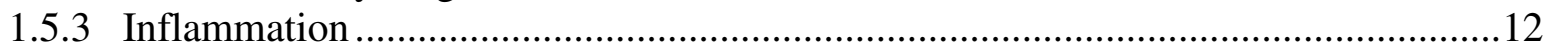

1.5.4 Brain atrophy and neuron loss........................................................................13

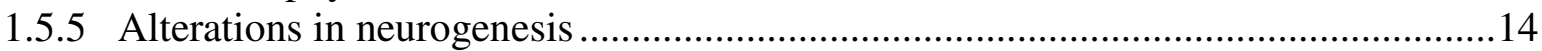

1.5.6 Motor impairment. Axonopathy ……………………………………………….....17

$1.6 \quad$ Pathomechanisms of Alzheimer's disease ………….................................................19

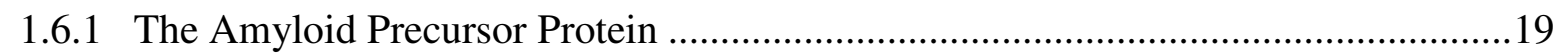

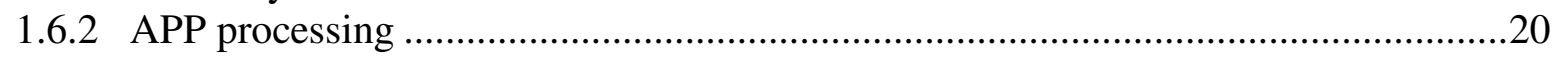

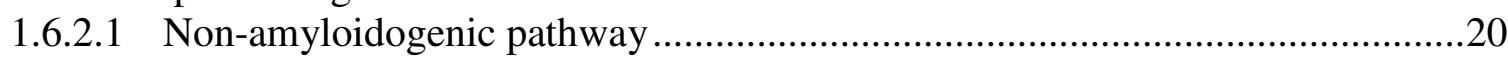

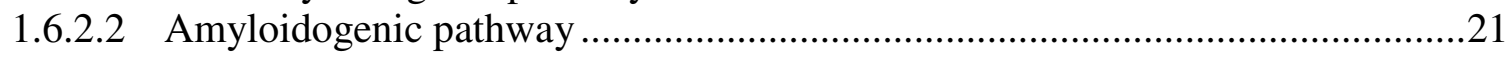

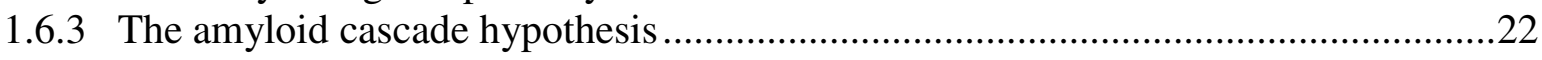

1.6.3.1 The classic amyloid cascade hypothesis ............................................................22

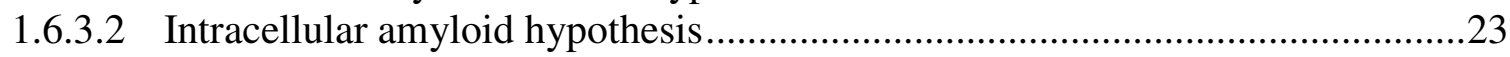

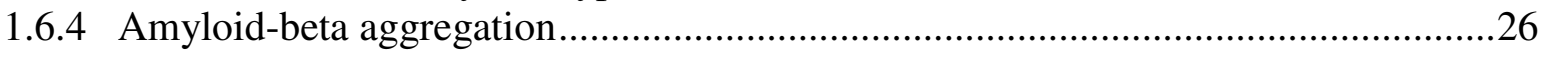

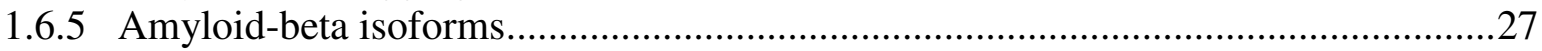

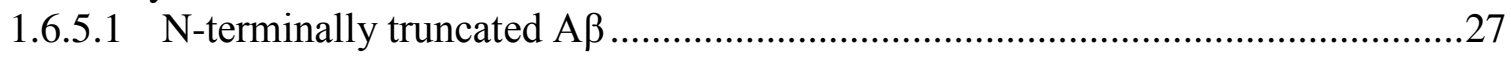

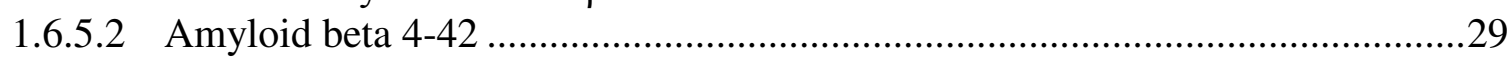

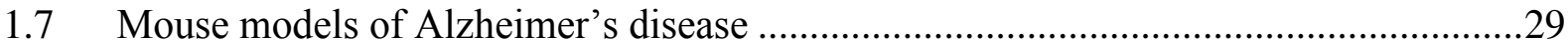

1.7.1 Mouse models and their relevance in AD research.................................................29

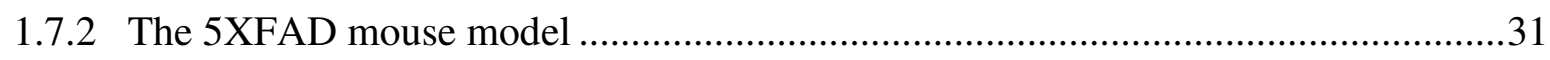

1.7.3 The Tg4-42 mouse model ...............................................................................3 


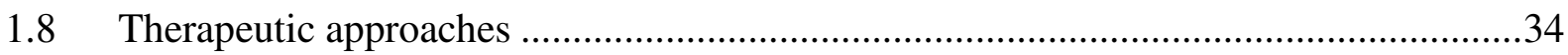

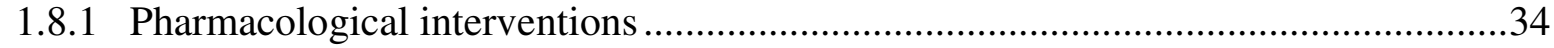

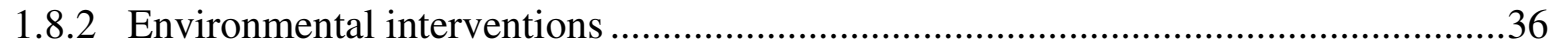

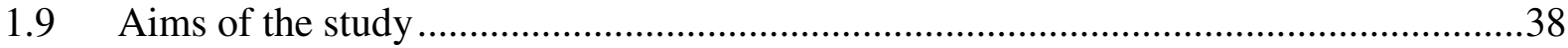

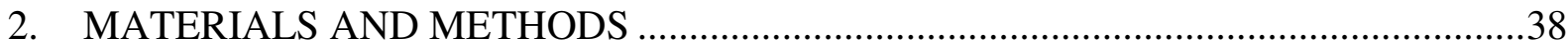

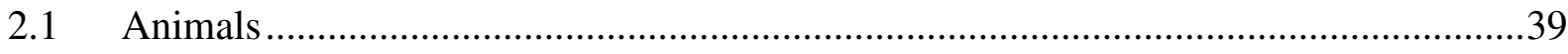

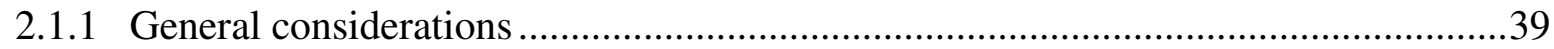

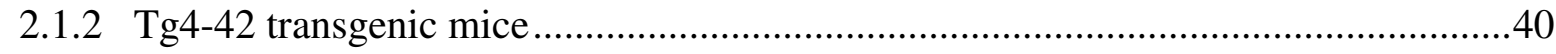

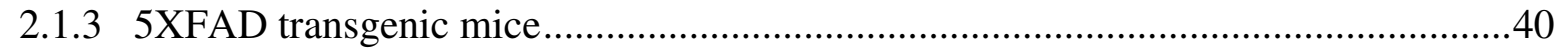

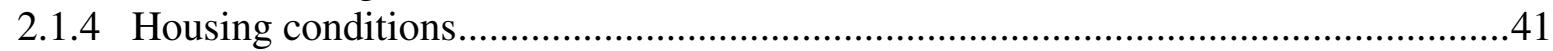

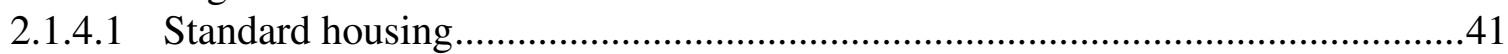

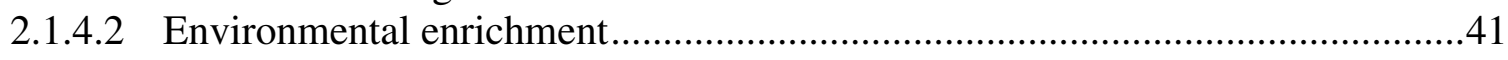

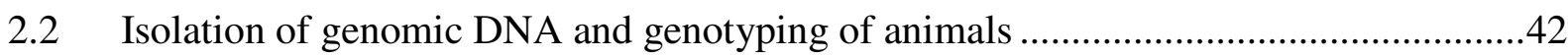

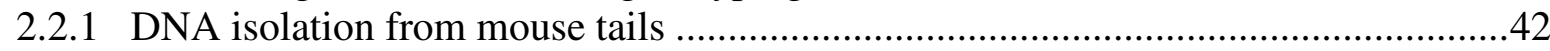

2.2.2 Determination of DNA concentration and purity ...............................................42

2.2.3 Genotyping of animals using polymerase chain reaction (PCR) ...........................42

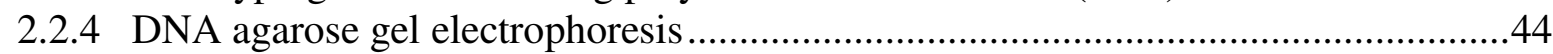

2.2.5 Quantitative Real-Time PCR Genotyping of 5XFAD mice …................................44

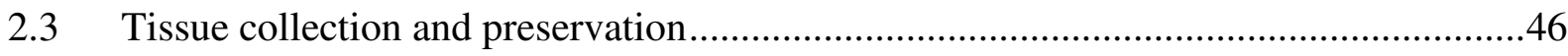

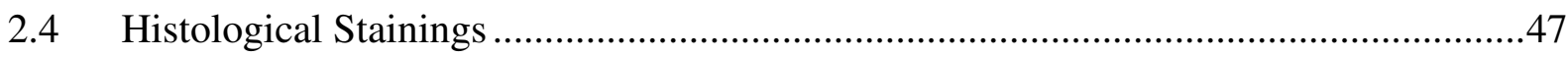

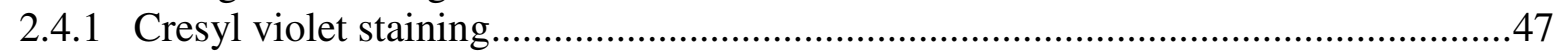

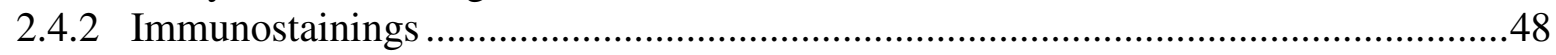

2.4.2.1 DAB immunohistochemistry of paraffin embedded section ............................48

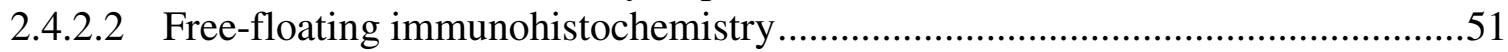

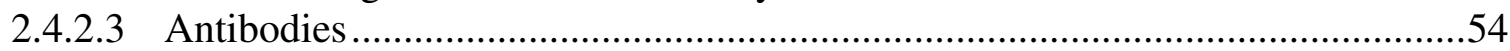

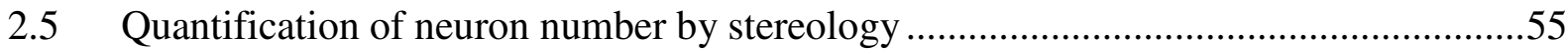

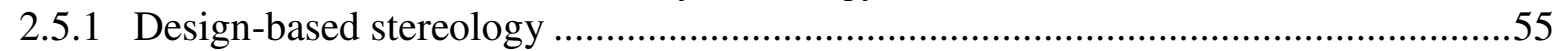

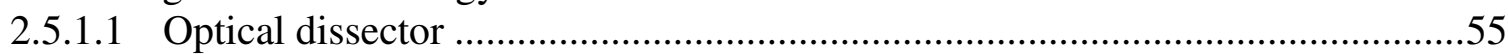

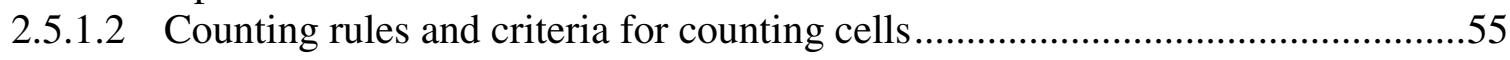

2.5.2 Quantification of the total neuron number in the granular cell layer of the dentate gyrus 57

2.6 Estimation of the volume of the dentate gyrus .........................................................59

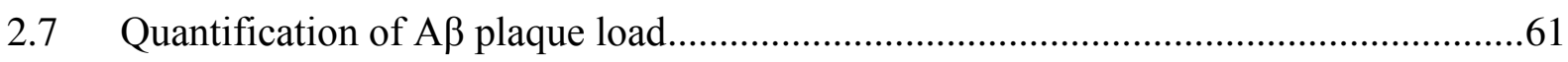

2.8 Quantification of doublecortin-positive neurons and plaque independent

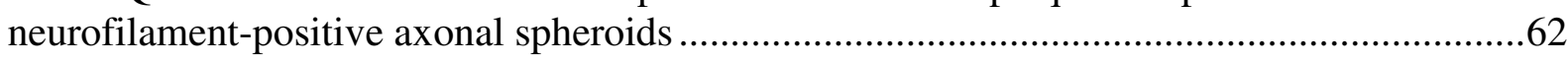

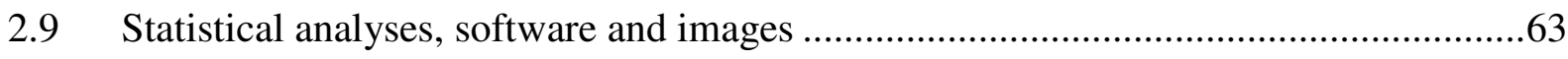

2.10 Lists of chemicals, reagents, kits and technical devices .........................................64 
3.1 Project I: Physical activity ameliorates neuron loss and increases neurogenesis in 6month-old Tg4-42 $2_{\text {hom }}$ mice.

3.1.1 Total neuron number: enriched environment and physical exercise attenuate neuron loss in Tg4-42 ${ }_{\text {hom }}$ mice.

3.1.2 Volume of the dentate gyrus: no significant difference between EE Tg4- $42_{\text {hom }}, \mathrm{SH}$ Tg4-42 $2_{\text {hom }}$, and WT mice.

3.1.3 Effects of environmental enrichment on adult subgranular neurogenesis: enriched environment triggers neurogenesis in $\mathrm{Tg} 4-42_{\text {hom }}$ mice

3.2 Project II: Gene dosage-dependent increase of amyloid pathology and axonal degeneration in $5 X_{\text {KAD }}$ hom mice.

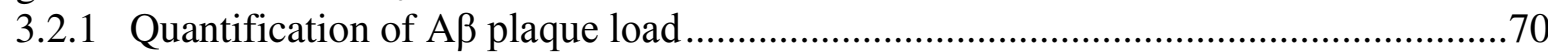

3.2.2 Quantification of neurofilament-positive axonal spheroids ...................................72

4. DISCUSSION.

4.1 Project I: Physical activity ameliorates neuron loss and increases neurogenesis in 6month-old Tg4-42 hom mice.

4.2 Project II: Gene dosage-dependent increase of amyloid pathology and axonal degeneration in $5 \mathrm{XFAD}_{\text {hom }}$ mice 


\section{List of figures}

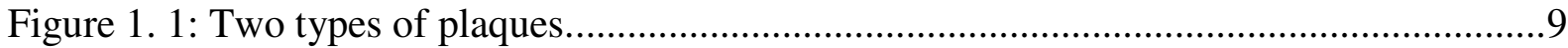

Figure 1. 2: Schematic overview about terms used to describe amyloid depositions ..............10

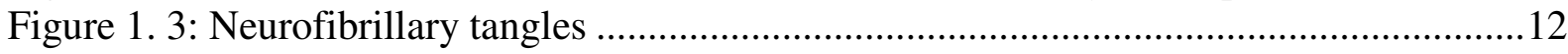

Figure 1. 4: Sagittal sections of brains: normal adult brain vs Alzheimer brain......................13

Figure 1. 5: Schematic sagittal view of a rodent brain pointing out the two neurogenic

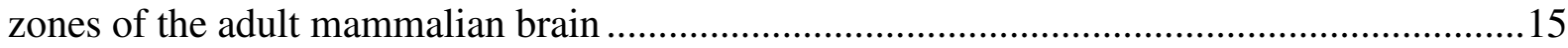

Figure 1. 6: Developmental stages in the course of adult hippocampal neurogenesis ............16

Figure 1. 7: Overview of physiological and environmental modulation of adult

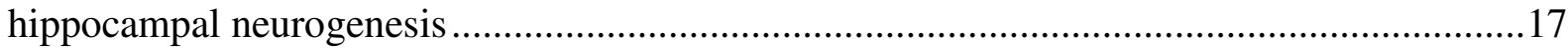

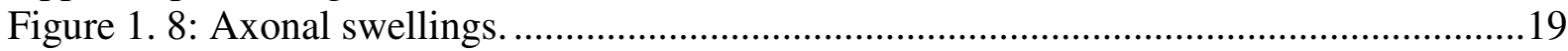

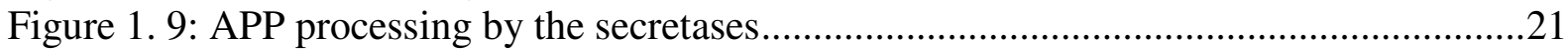

Figure 1. 10: Representation of the classic and the modified amyloid cascade hypothesis.. ...25

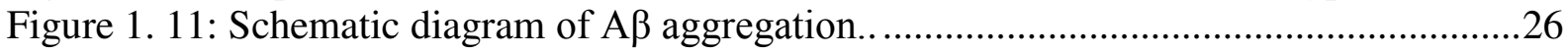

Figure 1. 12: The cleavage sites of enzymes involved in the degradation of full-length $A \beta$

and potential generation of $\mathrm{N}$-truncated $\mathrm{A} \beta$ isoforms. ......................................................28

Figure 1. 13: 5XFAD transgenes. Schematic diagram of 5XFAD APP and PS1 transgenes...32

Figure 1. 14: Genetic construct of the Tg4-42 transgene mouse...........................................33

Figure 2. 1: An example for Real-Time-PCR genotyping of 5XFAD mice...........................46

Figure 2. 2: Hippocampal granule cells expressing different markers.. ................................52

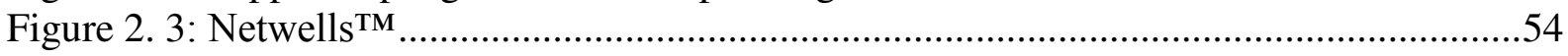

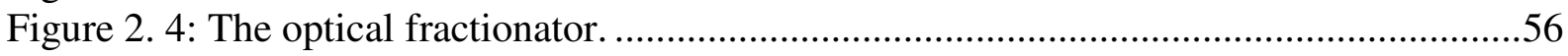

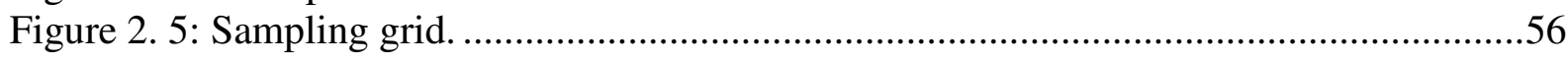

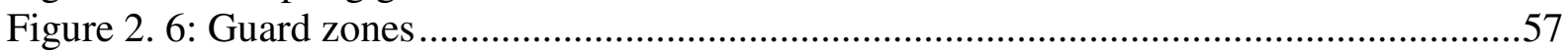

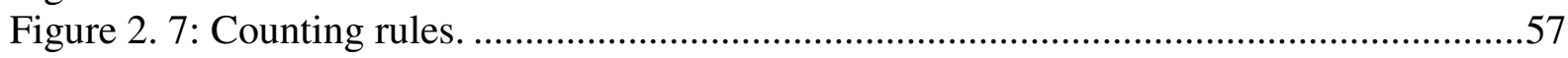

Figure 2. 8: Coronal slices of mouse brain highlighting dentate gyrus .................................59

Figure 2. 9: Cavalieri's principle in the brain region volume estimation. .............................60

Figure 2. 10: Brain regions, in which extracellular $A \beta$ load was evaluated. ...........................61

Figure 2. 11: An example of the application of the ellipsoid frame in the plaque load quantification.

Figure 3. 1: Quantification of neurons in GCL of the dentate gyrus using unbiased

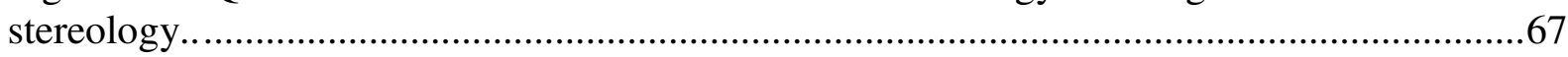

Figure 3. 2 Volume of the GCL of the dentate gyrus. .......................................................68

Figure 3. 3: Number of doublecortin-positive neurons in the SGZ of the dentate gyrus. .......69

Figure 3. 4: Illustration of extracellular $A \beta$ plaque load in cortex.. ...................................... 70

Figure 3. 5: Illustration of extracellular $A \beta$ plaque load in hippocampus, subiculum and

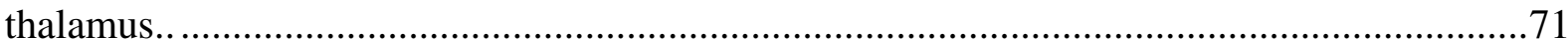

Figure 3. 6: Quantification of extracellular $A \beta$ plaque load in cortex, hippocampus,

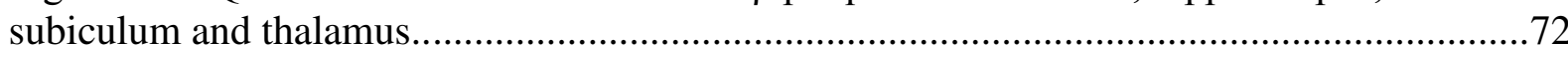

Figure 3. 7: Quantification of neurofilament-positive axonal spheroids in the pons and spinal cord. 


\section{List of tables}

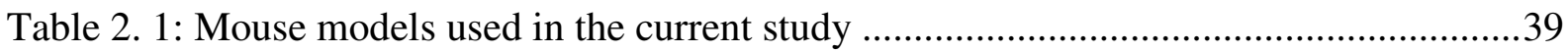

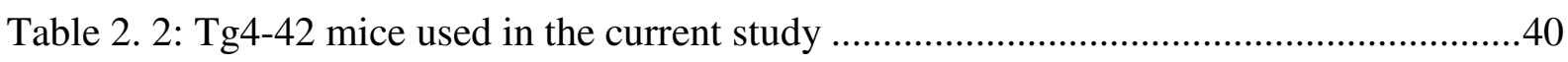

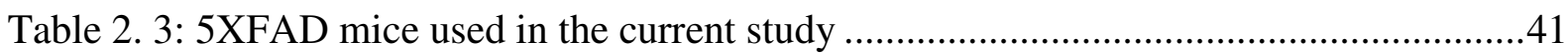

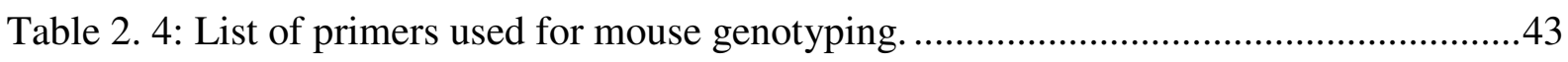

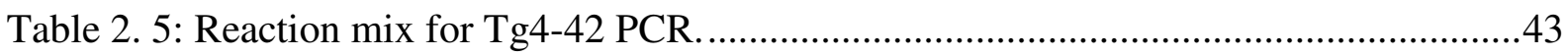

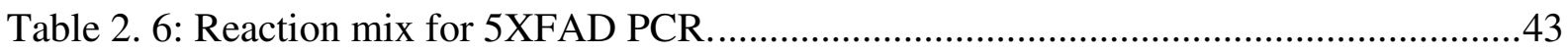

Table 2. 7: PCR cycling program for genotyping Tg4-42 and 5XFAD mice. .........................44

Table 2. 8: List of primers (Eurofins, Ebersberg, Germany) used for qRT-PCR .....................45

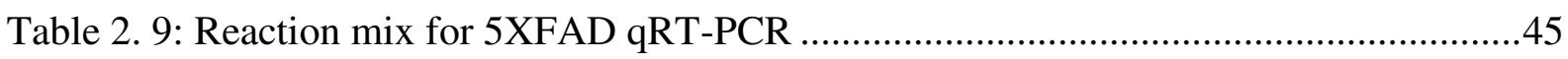

Table 2. 10: qRT-PCR cycling program for detecting $5 X_{F A D}{ }_{\text {hom }}$ mice ..................................45

Table 2. 11: Solutions required for cresyl violet staining ......................................................48

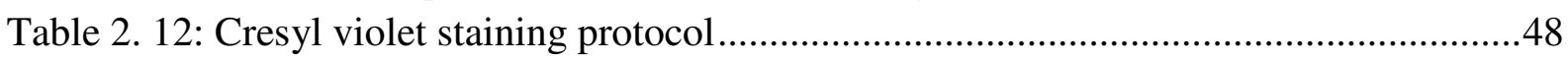

Table 2. 13: Solutions required for DAB immunohistochemistry .......................................50

Table 2. 14: DAB-Immunostaining protocol for paraffin-embedded sections on glass ...........50

Table 2. 15: DAB-Immunostaining protocol for free-floating frozen sections ........................53

Table 2. 16: Primary antibodies used for immunohistochemical stainings .............................54

Table 2. 17: Secondary antibodies applied for immunohistochemistry....................................54

Table 2. 18: Parameters for stereological analysis of the granular cell layer of the dentate

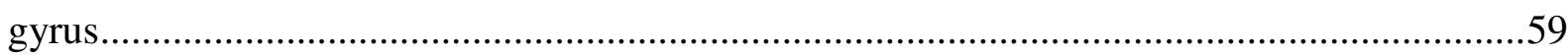

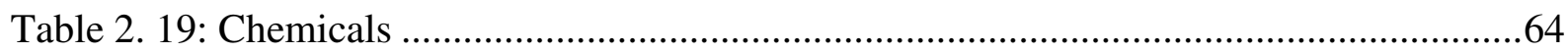

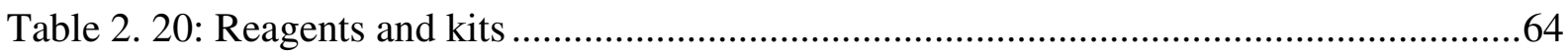

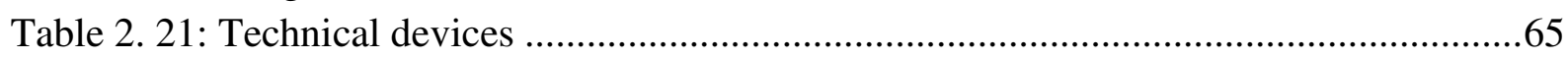




\section{List of abbreviations}

Please note that metric prefixes and units described in the International System of Units (SI) are not listed.

\begin{tabular}{|c|c|}
\hline 5XFAD & $\begin{array}{l}\text { transgenic mice (Tg6799) expressing five fa- } \\
\text { milial AD mutations }\end{array}$ \\
\hline $5 \mathrm{XFAD}_{\mathrm{hem}}$ & hemizygous 5XFAD \\
\hline 5 XFAD $_{\text {hom }}$ & homozygous 5XFAD \\
\hline $\mathrm{ABC}$ & avidin-biotin complex \\
\hline $\mathrm{AD}$ & Alzheimer's disease \\
\hline AICD & APP intracellular domain \\
\hline ANOVA & analysis of variance \\
\hline APP & amyloid precursor protein \\
\hline asf & area sampling fraction \\
\hline $\mathrm{A} \beta$ & amyloid beta \\
\hline $\mathrm{bp}$ & base pair(s) \\
\hline $\mathrm{CA}$ & cornu ammonis \\
\hline $\mathrm{CNS}$ & central nervous system \\
\hline $\mathrm{CR}$ & cognitive reserve \\
\hline DAB & 3,3'-Diaminobenzidin \\
\hline $\mathrm{DCX}$ & doublecortin \\
\hline $\mathrm{ddH}_{2} \mathrm{O}$ & distilled, deionized water \\
\hline $\mathrm{DG}$ & dentate gyrus \\
\hline DNA & deoxyribonucleic acid \\
\hline EDTA & ethylenediaminetetraacetic acid \\
\hline $\mathrm{EE}$ & enriched environment \\
\hline EOAD & early-onset Alzheimer's disease \\
\hline FAD & familial Alzheimer's disease \\
\hline FCS & fetal calf serum \\
\hline GCL & granular cell layer \\
\hline $\mathrm{H}_{2} \mathrm{O}_{2}$ & hydrogen peroxide \\
\hline hAPP & human APP \\
\hline $\mathrm{HCl}$ & hydrochloric acid \\
\hline hem & hemizygous \\
\hline hom & homozygous \\
\hline IVC & individually ventilated cages \\
\hline LOAD & late-onset Alzheimer's disease \\
\hline mAPP & murine APP \\
\hline MCI & mild cognitive impairment \\
\hline $\mathrm{MgCl}_{2}$ & magnesium chloride \\
\hline
\end{tabular}




\begin{tabular}{|l|l|}
\hline $\mathrm{m}$ & month \\
\hline min & minute \\
\hline $\mathrm{n}$ & number of animals \\
\hline NaCl & sodium chloride \\
\hline NFT & neuro-fibrillary tangles \\
\hline NIA & National Institute of Aging \\
\hline NIH & National Institutes of Health \\
\hline PBS & phosphate buffered saline \\
\hline PCR & polymerase chain reaction \\
\hline PFA & paraformaldehyde \\
\hline pGlu & pyroglutamate \\
\hline PSEN & presenilin \\
\hline qRT-PCR & real-time quantitative PCR \\
\hline rpm & revolutions per minute \\
\hline RT & room temperature \\
\hline SAD & sporadic Alzheimer's disease \\
\hline SDS & Sodium dodecyl sulfate \\
\hline SGZ & subgranular zone \\
\hline SH & standard housing \\
\hline ssf & section sampling fraction \\
\hline T & mean section thickness \\
\hline Tg4-42 & transgenic mice overexpressing A $\beta 4-42$ \\
\hline Tg4-42 hom & homozygous Tg4-42 \\
\hline TRH & thyreotropin releasing hormon \\
\hline Tris & Tris (hydroxymethyl) aminomethane \\
\hline tsf & thickness sampling fraction \\
\hline WHO & The World Health Organization \\
\hline WT & wild-type C57B6/J \\
\hline Z & dissector height \\
\hline & \\
\hline
\end{tabular}




\section{INTRODUCTION}

\subsection{Dementia}

Dementia is defined as "a progressive organic mental disorder characterized by chronic personality disintegration, confusion, disorientation, stupor, deterioration of intellectual capacity and function, and impairment of control of memory, judgment, and impulses" (Mosby's medical dictionary 2009, p. 501). Consciousness is usually not affected. Although dementia mainly affects older people, it is not considered to be a normal part of ageing. Dementia is characterized by "inexorably progressive deterioration in cognitive ability and capacity for independent living" (Prince et al. 2013, p. 64).

There are many causes of dementia. Some of them are drug or alcohol intoxication, pernicious anaemia, hyperthyroidism, paresis, subdural hematoma, benign brain tumour, hydrocephalus, insulin shock, or tumour of islet cells of the pancreas. These dementias can be reversed by treating the underlying condition. Quite the contrary, Alzheimer's disease, Pick's disease, vascular dementia, Lewy body dementia and other organic forms of dementia are generally considered irreversible, progressive, and incurable (Mosby's medical dictionary 2009).

Alzheimer's disease, vascular dementia, Lewy body dementia, and frontotemporal dementia are the most common underlying pathologies of dementia (Prince et al. 2013). Among these four pathologies, Alzheimer's disease is the most common cause of dementia and may contribute to 60-70\% of cases (World Health Organization 2015).

\subsubsection{Epidemiology of dementia}

Dementia is a very burdensome condition and is a top cause of disability and dependency among older people worldwide. It has an enormous physical, psychological, social and economic impact on caregivers, families and the society.

According to The World Health Organization (March 2015), 47.5 million people worldwide have dementia and there are 7.7 million new cases every year. The World Alzheimer Report 2015 states that these numbers will almost double every 20 years, reaching 74.7 million in 2030 and 131.5 million in 2050. These new estimates are 12-13\% higher comparing to the estimates from the World Alzheimer Report 2009. The prevalence of dementia in people aged 60 years and older ranges from $4.6 \%$ in Central Europe to $8.7 \%$ in North Africa and the Middle East. Rapidly increased prevalence of chronic diseases like dementia is associated with increasing life expectancy. Over the last century life expectan- 
cy increased from an average of 47 and 49 to 75 and 80 years of age for men and women respectively (Anderson and DeTurk 2002). Nowadays, there are almost 900 million people aged 60 years and older living worldwide (World Alzheimer Report 2015). In most countries, the rise in life expectancy is accompanied by declining fertility rates, so that older people also constitute an increasing proportion of total population $(\mathrm{NIH}$, National Institute of Aging 2011).

\subsubsection{The topicality of a problem of dementia and the importance of the ongoing research in this field}

The global cost of Alzheimer's disease and dementia is estimated to be US\$ 818 billion in 2015, which is equivalent to $1.09 \%$ of the entire world's gross domestic product. It is an increase of $35.4 \%$ comparing to 2010 (US\$ 604 billion in 2010) (World Alzheimer Report 2015).

In the context of the rapidly unfolding demographic changes, dementia becomes a health- and social-care priority for many countries. Governments in such high-income countries as the UK, France, Norway, USA, and South Korea are developing and implementing specific plans or strategies (Prince et al. 2013). However, in the World Alzheimer Report 2015 it was estimated that $58 \%$ of all people with dementia live in countries currently classified by the World Bank as low- or middle-income countries. These countries have fewer economic and professional resources to face the health and social care needs of their aging populations (World Alzheimer Report 2015). Particularly rapid increases in the numbers and proportion of older people are forecast for China, India, and Latin America (Prince et al. 2013).

The topic "Dementia" is also very present in mass media and arts. People search for the information about dementia, because they are afraid of it. They forced to deal with this problem, because they themselves, their close relatives or friends are affected (Peel 2014). Besides numerous articles in mass media, reports of the WHO, international and local Alzheimer's societies, many books were written and movies produced that deal with this problem. Among them are the American drama film „Still Alice“ (2014) based on Lisa Genova's 2007 novel of the same name, the German tragicomedy film "Head Full of Honey" (2014), the American science fiction film "Rise of the Planet of the Apes" (2011).

For all these reasons, it became important to intensify the research in the field of dementia and Alzheimer's disease in particular. In the last few decades, numerous substantial results in the field of Alzheimer's disease were achieved. The dominant familial Alzheimer (FAD) mutations, genetic and environmental risk factors for sporadic AD were identified, different transgenic animal models were created (Woodruff-Pak 2008), the drugs that can slow down the evolution of symptoms were developed (Rafii and Aisen 2009), the endogenous neurotoxic species were characterised and the subsequent clinical trials of immunization against them were performed (Kayed and Jackson 2009; Wisniewski and Konietzko 2008). It was also shown that a modulation of environmental factors and improving physical conditions has been efficient in the prevention of AD. Understanding the effects of lifestyle factors on the disease course might help directing therapeutic approaches (Arenaza- 
Urquijo et al. 2015). Moreover, some epidemiological studies have shown that higher education levels could postpone the onset of the dementia syndrome (Paradise et al. 2009) and act as protective factors against the cognitive symptoms of AD (Roe et al. 2007). The underlying mechanism of this phenomenon is supposed to be a so called cognitive reserve (Arenaza-Urquijo et al. 2015). The cognitive reserve implies that persons with more cognitive capacities can cope longer and more effective with the pathological manifestations of AD due to a higher variability at the level of brain networks (Stern 2002; Stern 2006; Stern 2009).

The lifestyle-based interventions and increasing intellectual capacities by cognitive and physical exercise are potential low-cost preventive measures that could delay the onset of $\mathrm{AD}$ and help maintaining cognitive performance of the patients.

\subsection{Alzheimer's disease: history and epidemiology}

In 1906, at the $37^{\text {th }}$ meeting of the Society of Southwest German Psychiatrists in Tübingen, the German psychiatrist Alois Alzheimer reported about one peculiar case of a patient, the 51 years-old Auguste Deter, from the Frankfurt Asylum. He observed this patient from 1901 till her death in 1906 (Goedert and Spillantini 2006). Auguste Deter suffered from cognitive and language deficits like memory disturbance, loss of orientation, delusions, auditory hallucinations as well as aggressive behaviour (Alzheimer 1907). After her death, Alzheimer took the patient's brain to Munich and investigated it using staining technics in Emil Kraepelin's lab. Together with other scientist of Kraepelin's lab, Alois Alzheimer identified macro- and microscopic alterations such as brain atrophy, amyloid plaques and neurofibrillary tangles. The case of Auguste Deter reported on the $3^{\text {rd }}$ November 1906 in Tübingen was the first reported case of the disease with this characteristic combination of symptoms, and the first time that the pathology and the clinical symptoms of presenile dementia were presented together (Maurer and Maurer 2003). In his textbook in 1910, Emil Kraepelin coined the term “Alzheimer's disease” (Kraepelin 1910) and thereby established the name, which is still used today.

Alois Alzheimer died only 9 years after he described the case of Auguste Deter (Maurer and Maurer 2003). He might have hardly imagine that 100 years later the most common neurodegenerative disease would be called after him, and speaking about dementia we would mostly speak about Alzheimer's disease.

Alzheimer's disease is the most common cause of dementia with nearly 30 million people worldwide suffering from it (according to the WHO 2015). Currently, 1.5 million people suffer from dementia in Germany. According to German Alzheimer's Association (Deutsche Alzheimer Gesellschaft), almost two-thirds of them are suffering from Alzheimer's disease. Due to the ageing population, the number of dementia patients increases continuously, with around 300000 new patients every year. Unless there will be a decisive breakthrough in prevention and therapy, the number of people affected by dementia in Germany will increase, reaching 3.0 million in 2050 (Deutsche Alzheimer Gesellschaft 2014). 


\subsection{Clinical aspects of Alzheimer's disease}

\subsubsection{Progression of disease}

The progression of Alzheimer's disease (AD) is slow and results in a progressive decline of memory functions in combination with non-cognitive symptoms like personality and mood changes finally leading to inability to perform everyday activities (Alzheimer's Association 2012). The average development from mild and moderate AD to severe AD occurs in the average time of 7 to 10 years (Alzheimer's Association 2012; Holtzman et al. 2011). Early-onset AD happens to people who are younger than age 65 . Often, they are in their 40s or 50s when AD is diagnosed in them. Most patients suffering from the earlyonset $\mathrm{AD}$ have familial $\mathrm{AD}$ (an inherited form, see 1.3.2). However, the majority of $\mathrm{AD}$ cases are referring to late-onset $\mathrm{AD}$, which occurs sporadically in people being 65 years and older. Late-onset AD arises from a complex of brain changes and impairments that occur over decades (NIH, National Institute of Aging 2015).

In 2011, the Alzheimer's Association and the National Institute on Aging (NIA) proposed new guidelines for the classification and diagnosis of AD. The course of AD was divided in three stages: preclinical AD, mild cognitive impairment (MCI) due to $\mathrm{AD}$ and dementia due to AD (Albert et al. 2011; Jack et al. 2011; McKhann et al. 2011; Sperling et al. 2011). Preclinical AD is a newly defined stage of the disease. It reflects the evidence that measureable biomarker changes in the brain may occur years before symptoms affecting memory, behaviour or thinking can be detected. It was estimated that neurodegeneration and changes in brain start already 20 years before AD can be diagnosed (Blennow et al. 2006). During the preclinical phase, patients do not have any measurable clinical symptoms, whereas individuals with mild cognitive impairment show a visible decline compared to same-aged healthy individuals (Alzheimer's Association, 2012). The conversion of MCI cases into dementia due to AD occurs each year with a rate of 10-15\%. In these cases MCI is considered to be an early stage of AD (Petersen 2004; Visser et al. 2005). Later, patients develop dementia due to $\mathrm{AD}$. This stage is characterized by severe memory decline, language dysfunction, personality and behaviour changes, motor impairments and loss of visuospatial abilities (Alzheimer's Association 2012; Holtzman et al. 2011).

$\mathrm{AD}$ affects each person in a different way, also depending upon the person's personality before acquiring the disease. The progression of disease can be divided in three stages. The onset of the early stage of AD is gradual and can often be overlooked. The common symptoms are forgetfulness, losing track of time and becoming lost in formerly familiar places. At the middle stage, the symptoms are clearer and more restricting. These can include regularly forgetting recent events, names and faces, becoming increasingly repetitive, getting lost at home, and having problems finding the right words. Mood and behaviour changes such as apathy and irritability occur. Patients lose confidence and need help with personal care. At the late stage of AD, patients are inactive and totally dependent on caregivers. Memory disturbances are severe and physical symptoms are obvious. At this stage, symptoms include being unaware of the time and place, having difficulty recognizing relatives and friends, having an increased need for assisted self-care, having difficulty 
walking, becoming unsteady on the feet and falling down, showing behaviour changes that may escalate and include aggression. Moreover, patients can become sad or depressed. At the late stage, people may also experience hallucinations. Anxieties or phobias are also common (World Health Organization 2015).

At the terminal stage of the disease patients are even unable to perform basic motor functions like swallowing. This accumulation and progressive severity of symptoms is ultimately fatal and finally leads to death. Although dementia is a dramatically lifeshortening illness, other conditions or illnesses may actually cause a person's death. For example, pneumonia is listed as the ultimate cause of death in up to two-thirds of people with dementia (Holtzman et al. 2011; Wada et al. 2001).

\subsubsection{Genetic factors}

As mentioned above, there are two forms of AD described: a familial $\mathrm{AD}$ and a sporadic $\mathrm{AD}$. A familial $\mathrm{AD}(\mathrm{FAD})$ is an inherited form and constitutes approximately $1 \%$ of the disease cases (Zetterberg and Mattsson 2014). The majority of cases (99\%) is classified as sporadic $\mathrm{AD}(\mathrm{SAD})$.

Persons originating from families, members of which have had early-onset AD (EOAD) over at least two generations, have a high risk of developing FAD (Selkoe 2001). Familial AD typically develops before the age of 65 years (early-onset) and is caused in the first place by overproduction of Abeta-protein $(A \beta)$ due to mutations either in the amyloid precursor protein (APP) gene or genes encoding presenilin 1 (PSEN1) or presenilin 2 (PSEN2). Approximately 50\% of the FAD patients carry mutations in one of the three genes PSEN1, PSEN2, or APP (Shea et al. 2015). PSEN1, PSEN2 and APP are located on the chromosome 14, 1 and 21, respectively (Masuhr and Neumann 2007; Rogaev et al. 1995; Sherrington et al. 1995).

PSEN1 and PSEN2 are essential components of the $\gamma$-secretase complexes. $\gamma$-secretase is responsible for cleavage and release of $A \beta$ peptides of various lengths (see 1.6.2.2). In their non-mutated forms, presenilins play a variety of physiological roles such as cell proliferation (Wines-Samuelson and Shen 2005), cellular differentiation (Baumeister 1999), and neurotransmitter release (Zhang et al. 2009a).

The first described causes of FAD were mutations in the APP gene (Goate et al. 1991). APP mutations are located close to the secretase cleavage sites. For example, the first described APP mutation, London mutation $\mathrm{APP}_{\mathrm{V} 717 \mathrm{I}}$, is located on the $\gamma$-secretase cleavage site. This mutation replaces valine with isoleucine at protein position 717 . This mutation increases the $A \beta 42 / A \beta 40$ ratio by increasing $A \beta 42$ levels, while $A \beta 40$ levels are not significantly affected (Eckman et al. 1997; Jonghe et al. 2001; Theuns et al. 2006; Herl et al. 2009).

The Swedish mutation $\mathrm{APP}_{\mathrm{K} 670 \mathrm{~N}, \mathrm{M} 671 \mathrm{~L}}$ is the only known mutation near the $\beta$-secretase cleavage site. It is a double mutation, resulting in a substitution of two amino acids, lysine and methionine to asparagine and leucine, respectively. This well-known mutation was found in two large Swedish families, which were connected genealogically. Affected indi- 
viduals presented first with memory loss and all met diagnostic criteria for AD (Mullan et al. 1992). The Swedish mutation increases the total $A \beta$ levels (Haass et al. 1995).

Another APP mutation, APP ${ }_{1716 \mathrm{~V}}$ or so-called Florida mutation was first reported in an American patient with suspected Alzheimer's disease. She had a family history of dementia with a mean age of onset of about 53 years (Eckman et al. 1997).

Another form of the APP-associated AD has been observed in patients with Down's syndrome. An increased $A \beta$ peptide production is caused by an increased dosage of chromosome 21 genes. The plasma levels of amyloid beta proteins $A \beta_{1-40}$ and $A \beta_{1-42(43)}$ are both elevated in Down's syndrome (Tokuda et al. 1997).

Even though, the autosomal dominant forms of $\mathrm{AD}$ are responsible only for less than 1 $\%$ of the total number of AD cases, the discovery of mutated genes, which trigger the development of the disease, is crucial for understanding the pathological processes. In particular this concerns research based on animal models.

The majority of AD cases occur in elderly ( $>65$ years) and are commonly referred to as late-onset $\mathrm{AD}$ (LOAD). There are multiple environmental and genetic risk factors contributing to the developing of the LOAD (see 1.3.4), but impairment in $\mathrm{A} \beta$ clearance is probably a major contributor to disease pathogenesis (Liu et al. 2013).

\subsubsection{Risk factors for Alzheimer's disease}

The most significant risk factor for sporadic Alzheimer's disease is aging (Blennow et al. 2006). 12,5\% people older than 65 years and $45 \%$ of the people older than 85 suffer from AD. Despite its high prevalence among older people, the disease is not considered to be a normal aging process (Alzheimer's Association, 2012).

Apart from aging, gender is an important risk factor for AD. The incidence of the disease is higher in women than in men (Alzheimer's Association, 2015), and this is not only due to higher life expectancy. Interestingly, mitochondria in young females are protected against amyloid-beta toxicity. They generate less reactive oxygen species and release less apoptogenic signals than mitochondria in males (Viña and Lloret 2010). However, this protective effect is lost in mitochondria in old post-menopausal females.

Thus, it is held that estrogenic compounds may protect against mitochondrial toxicity of amyloid-beta. This protective effect has been observed in a number of laboratories worldwide and suggests a method for treating or preventing AD by estrogenic compounds (Viña and Lloret 2010).

Epidemiological studies have suggested further risk factors for sporadic AD: vascular diseases like atherosclerosis, hypercholesterinaemia, coronary heart disease and heart failure (Kivipelto et al. 2001; Kivipelto et al. 2005; Qiu et al. 2006). In addition, several studies showed that obesity, smoking and type II diabetes also increase the risk of developing AD (Kivipelto et al. 2005; Prince et al. 1994). Head injury and traumatic brain injuries are also regarded as risk factors for AD (McCullagh et al. 2001; Plassman et al. 2000; Sivanandam and Thakur 2012). Severe head injury leads to beta-amyloid protein deposition in the brain (Roberts et al. 1994), and increases a number of beta APP-immunoreactive neurons in the entorchial cortex (McKenzie et al. 1994). 
Moreover, carrying at least one copy of the ApoE4 allele increases the risk for sporadic AD (Corder et al. 1993). The Apolipoprotein E has a variety of functions. It is involved in microtubule stability, cell survival, cholesterol transport and amyloid deposition (Herz and Beffert 2000). The Apoprotein E gene is mapped to chromosome 19q. This gene is polymorphic and has three major alleles: ApoE2, ApoE3 and ApoE4. The presence of $t \varepsilon 4$ allele is associated with increased risk for both early- and late-onset AD. Compared with individuals with an $\varepsilon 3 / \varepsilon 3$ genotype, individuals with one copy of the $\varepsilon 4$ allele developed AD much more frequently. Genome-wide association studies have verified that the $\varepsilon 4$ allele of ApoE is the most significant genetic risk factor for sporadic AD (Liu et al. 2013). Furthermore, there are indications that Apolipoprotein E $\varepsilon 2$ allele protect against late-onset AD (Corder et al. 1994).

Concearning protective factors, there are a variety of studies confirming that a healthy and physically active lifestyle together with cognitive stimulating activities and a Mediterranean diet reduce risk for Alzheimer's disease and protects against it (Fratiglioni et al. 2004; Gu et al. 2010; Hall et al. 2009) (see 1.8.2).

\subsection{Diagnosis of Alzheimer's disease}

Currently, a definite diagnosis of $\mathrm{AD}$ can only be confirmed by examining the brain post mortem. In 2011, the National Institute on Aging (NIA) provided guidance for documenting and reporting Alzheimer's-related brain changes observed during autopsy. According to this guideline, there are several characteristic lesions, which can be attributed to AD. For a neuropathological diagnosis of $\mathrm{AD}$, neuropathological change has to be ranked along three essential parameters: Aß plaques, Neurofibrillary tangles (NFTs) and Neuritic plaques (Braak and Braak 1991; Hyman et al. 2012; McKhann et al. 1984).

Prior to the patient's death, cognitive and behaviour changes as well as the family history of a patient are used to diagnose possible AD (Alzheimer's Association, 2015).

In 1983, a group was convened by the National Institute of Neurological and Communicative Disorders and Stroke (NINCDS) and the Alzheimer's Disease and Related Disorders Association (ADRDA) for the purpose of establishing criteria for the clinical diagnosis of AD. The report of the NINCDS-ADRDA work group (McKhann et al. 1984) contains issues of AD medical history, clinical examination, neuropsychological testing, and laboratory assessments. The criteria in this report (NINCDS-ADRDA criteria) have been quite successful during the quarter of the century. However, these criteria also required revision. Therefore, in 2011, the National Institute on Aging and the Alzheimer's Association convened a workgroup to revise the 1984 criteria for AD dementia. The NINDS-ADRDA criteria had to be reviewed and then updated reflecting modern innovations in clinical diagnosis, imaging, and laboratory assessment (McKhann et al. 2011). The National Institute on Aging and the Alzheimer's Association workgroup proposed the following terminology for classifying individuals with dementia caused by AD: (1) Probable AD dementia (dementia may be due to another cause), (2) Possible AD dementia (no other cause for dementia can be found), and (3) Probable or possible AD dementia with evi- 
dence of the $A D$ pathophysiological process. (1) and (2) can be used in all clinical settings, (3) is intended for research purposes.

A detailed description of core clinical criteria for all-cause dementia, probable AD and possible $\mathrm{AD}$ is highlighted in "The diagnosis of dementia due to Alzheimer's disease: Recommendations from the National Institute on Aging and the Alzheimer's Association workgroup" (McKhann et al. 2011). In this chapter, I would like to mention only some exclusion criteria for AD. For example, the diagnosis of probable AD dementia should not be applied when there is evidence for substantial concurrent diseases of the following types (McKhann et al. 2011):

(1) cerebrovascular disease (a history of a stroke, which is temporally related to the onset of cognitive decline, or the presence of multiple or extensive infarcts or severe white matter hyperintensity burden);

(2) dementia with Lewy bodies;

(3) behavioural variant frontotemporal dementia;

(4) specified aphasia;

(5) other concominant active neurological disease;

(6) use of medication that could have a strong effect on cognition.

A variety of tests has been developed to diagnose AD. Among them, there are testing procedures such as the Mini-Mental Stage Examination (Folstein et al. 1975), the ClockDrawing Test (Aprahamian et al. 2010; Sunderland et al. 1989) or the Cambridge Cognitive Examination (Schmand et al. 2000; Martinelli et al. 2014). For a probable diagnosis of $\mathrm{AD}$, severe deficits that affect the patient in carrying out daily activities are essential (American Psychiatric Association 1995). Besides memory impairment, patients must show such symptoms as agnosia, aphasia, apraxia or impairments in executive functions (Waldemar et al. 2007). The diagnosis can be supported by neuroimaging (Magnetic Resonance Tomography, Computer Tomography, or Positron-Emission Tomography with radiolabeled glucose (FDG-PET ${ }^{1}$ ) or an amyloid-binding dye (PiB-PET ${ }^{2}$ ) (Ballard et al. 2011; Blennow et al. 2006; Perrin et al. 2009; Schroeter et al. 2009). With these methods, alternative causes of dementia like brain tumours, hydrocephalus or subdural hematomas can be excluded. In addition, analysis of biomarkers in the cerebrospinal fluid has been established for diagnosis of MCI and AD. Detection of reduced levels of $A \beta_{x-42}$ and increased levels of Tau and phosphorylated Tau can support a diagnosis (Fiandaca et al. 2014; Mattsson et al. 2009; Perrin et al. 2009).

An early diagnosis of AD is very important. It gives people opportunity to participate in clinical trials and research studies to test possible new treatments. If the disease is treated at the early stage, important capabilities can be preserved for some time. An early diagnosis also helps patients and their families to plan for the future.

\footnotetext{
${ }^{1}$ Fluordesoxyglucose positron emission tomography

${ }^{2}$ Pittsburgh compound B-positron emission tomography. PiB is a radioactive analog of thioflavin $\mathrm{T}$.
} 


\subsection{Neuropathological hallmarks of Alzheimer's disease}

\subsubsection{Amyloid-beta deposition}

Amyloid-beta deposition is one of the major neuropathological hallmarks of AD. A $\beta$ depositions are specific formations composed of extracellular accumulation of the Amyloid-beta peptide $(A \beta)$, which is derived from cleavage of Amyloid-Precursor-Protein (APP) (Holtzman et al. 2011; Serrano-Pozo et al. 2011). The particular A $\beta$ species are classified with respect to their corresponding amino acid sequence e.g. $\mathrm{A} \beta_{\mathrm{X} 1-\mathrm{X} 2}$ is an $\mathrm{A} \beta$ peptide, which starts from the $\mathrm{N}$-terminal amino acid $\mathrm{X} 1$ and ends with the $\mathrm{C}$-terminal amino acid X2.

The term "senile plaque" used for decades for description of $A \beta$ accumulation is very unprecise. Duyckaerts et al. (2009) suggested in the review "Classification and basic pathology of Alzheimer disease" limit the use of this term. Duyckaerts et al. (2009) distinguish between mature $A \beta$ deposition, neuritic plaque (especially when there is no referring to the technique that has been used to reveal the lesion), and other extracellular accumulation of $\mathrm{A} \beta$, called deposits. For clarity, speaking about $\mathrm{A} \beta$ deposits, it should be mentioned, how they have been revealed e.g. A $\beta$ deposits can be revealed by anti-A $\beta$ antibodies, and high density amyloid deposits can be revealed by Congo red or Thioflavin-S staining. A second possible qualifying term for deposits may be the description of their shape: diffuse, focal or stellate $A \beta$ deposits (Delaère et al. 1991; Duyckaerts et al. 2009) (Figure 1.2).

In this thesis, I would like to distinguish between diffuse $\mathrm{A} \beta$ deposits, calling them diffuse plaques, and neuritic plaques.
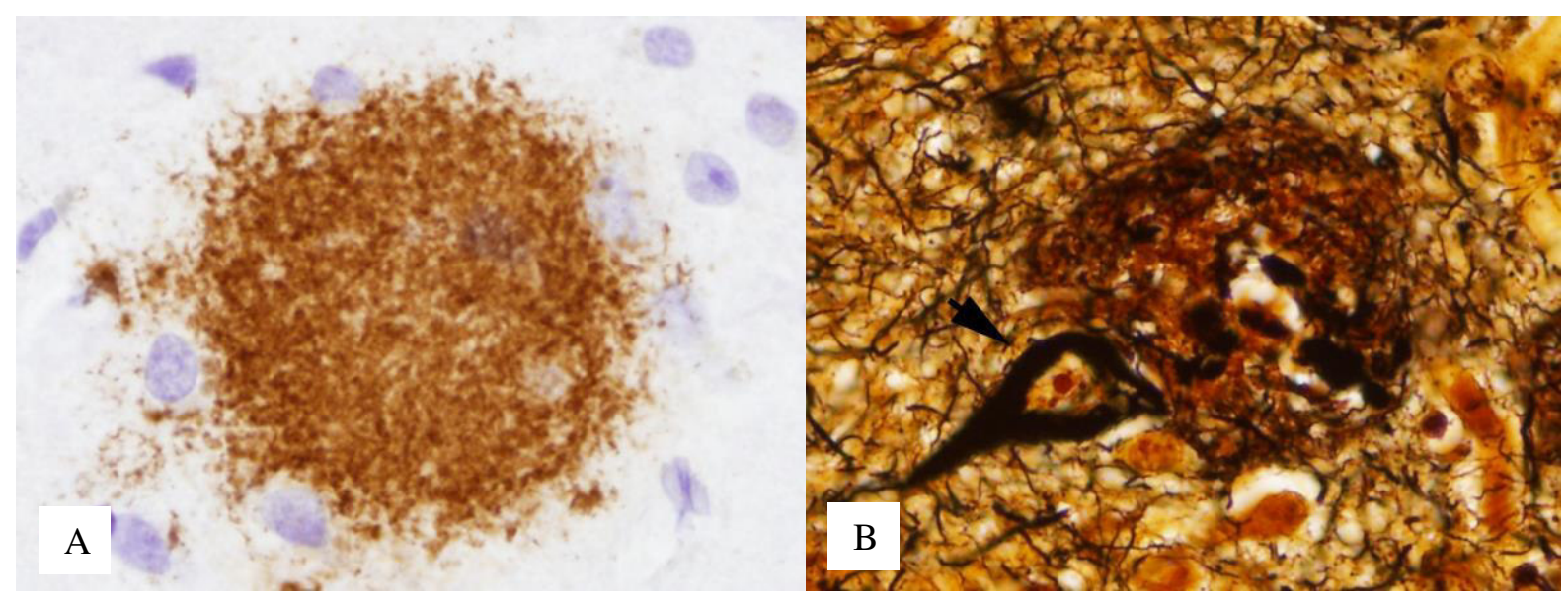

Figure 1. 1: Two types of amyloid plaques. (A) Diffuse plaques (brownish cloud). A $\beta$ immunohistochemistry. (B) Neuritic plaque (big dark clew in the middle of the image) with neurofibrillary tangle (arrow). Neuritic plaques can contain reactive astrocytes and microglia, and are surrounded by swollen, degenerating axons and dendrites. Figures reproduced from www.neuropathology-web.org 
Diffuse plaques (Figure 1.1A) are composed of non-fibrillary depositions of $A \beta$ with almost no detectable neuritic dystrophy. They can be revealed using A $\beta$ immunohistochemistry. Their diameter can vary from $50 \mu \mathrm{m}$ to several hundred micrometres (Duyckaerts et al. 2009; Yamaguchi et al. 1988). Diffuse plaques are also found in healthy older individuals. This led to the hypothesis that an increase of the plaque load is associated with preclinical AD (Dickson et al. 1992; Knopman et al. 2003; Vlassenko et al. 2011).

Neuritic plaques (Figure 1.1B) are extracellular A $\beta$ plaques which consist of highly aggregated fibrillary $A \beta$. These plaques are surrounded by swollen, degenerating axons and dendrites (Nelson et al. 2009). Plaque density and size can differ (with diameter from 10 to $120 \mu \mathrm{m}$ ) (Thomas and Fenech 2007). Neuritic plaques can be stained with $\beta$-sheet staining dyes such as Thioflavin-S and Congo red indicating their fibrillary structure (Serrano-Pozo et al. 2011). In the immediate vicinity to neuritic plaques, a variety of neuropathological changes is observed: neuritic dystrophies, degenerated neurons, as well as astroand microgliosis (see 1.5.3) (Holtzman et al. 2011; Lenders et al. 1989; Masliah et al. 1990; Pike et al. 1995b; Selkoe 2011; Urbanc et al. 2002). Besides A $\beta$, other proteins such as APP, Tau and Ubiquitin are found in neuritic plaques ( $\mathrm{Su}$ et al. 1998; Duyckaerts et al. 2009).

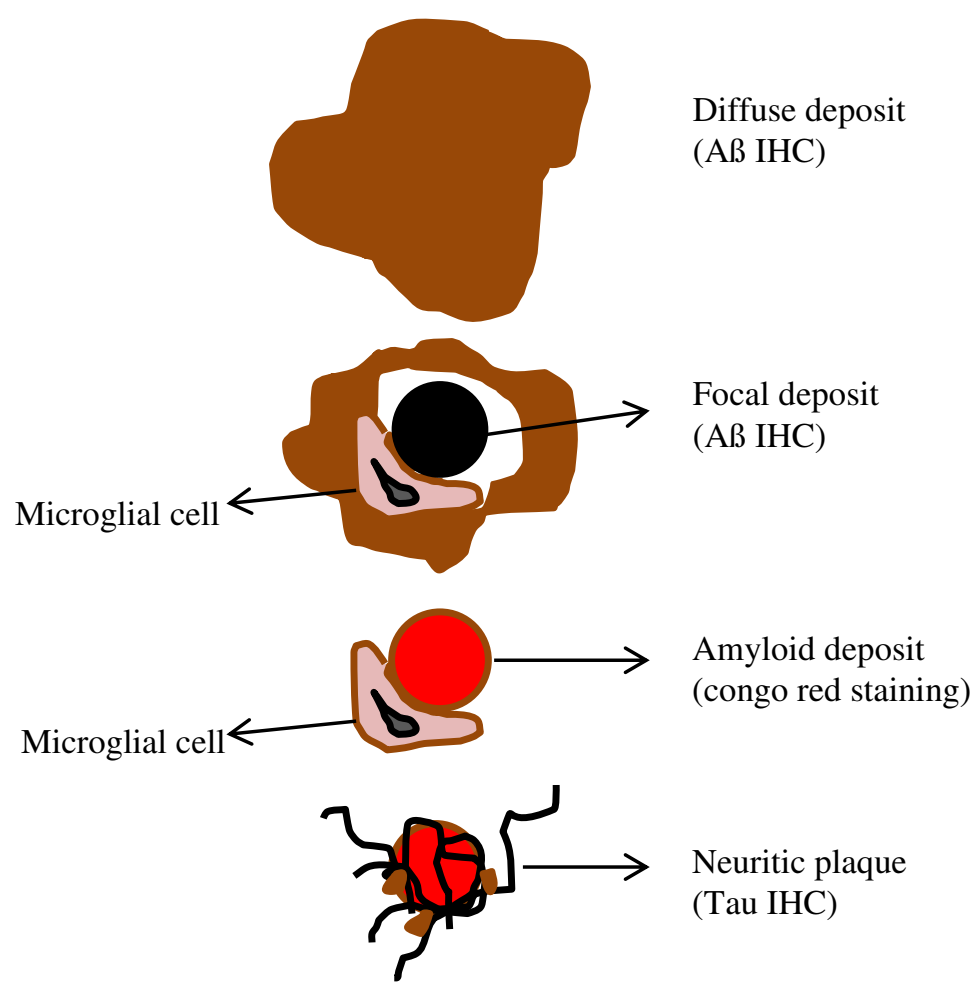

Figure 1. 2: Schematic overview over terms used to describe amyloid depositions. Central aspects of A $\beta$ deposition revealed by different techniques are illustrated. The terms applied to qualify the focal deposits depend on the technique used to reveal them, for instance $A \beta$ - and Tau-immunohistochemistry (IHC), Congo red or Thioflavin-S. Tau-immunohistochemistry only reveals neuritic plaques. The vertical position of the figures should represent the sequence of events leading to the neuritic plaque. Stellate deposits are not shown. Figure created after Duyckaerts et al. 2009, p. 7.

The amyloid deposition typically starts in the neocortex, and then affects the hippocampus, basal ganglia and cerebellum (Serrano-Pozo et al. 2011). In the final stage of AD, 
neuritic plaques are also found in subcortical structures such as the brainstem (Aldwin and Gilmer 2013; Arnold et al. 1991; Thal et al. 2002).

In spite of the fact that plaques are a key feature in the diagnostic of $\mathrm{AD}$, the absolute plaque load correlates poorly with cognitive impairment and disease stage (Arriagada et al. 1992; Billings et al. 2005; Giannakopoulos et al. 2003; Schaeffer et al. 2011; Villemagne et al. 2011). Approximately $80 \%$ of the AD patients also show the symptom of amyloid deposition in blood vessels, called Cerebral Amyloid Angiopathy (CAA) (Glenner and Wong 1984).

\subsubsection{Neurofibrillary tangles}

Neurofibrillary tangles (NFT) are intracellular formations consisting of hyperphosphorylated Tau protein organized in paired helical filaments (Kidd 1963; Grundke-Iqbal et al. 1986; Lee et al. 1991) (Figure 1.3). Tau is a phosphoprotein expressed in all nucleated cells and is abundant in neurons (Duyckaerts et al. 2009; Galimberti and Scarpini 2012). Already Alois Alzheimer mentioned NFTs in his report as "intraneuronal filamentous inclusions" (Alzheimer 1907). Tau is a microtubule-associated protein and considered to be a significant factor in the axonal growth, development of neuronal polarity, and the maintenance of microtubule dynamics (Higuchi et al. 2002). In its normal function, Tau is involved in the organization of cytoskeleton (Drechsel et al. 1992; Gustke et al. 1994; Weingarten et al. 1975; Witman et al. 1976), whereby it binds to tubulin assembling and stabilizing microtubules (Goedert and Spillantini 2006). These functions of tau are primarily regulated by the activities of protein kinases and phosphatases (Higuchi et al. 2002). Hyperphosphorylation of Tau reduces tubulin's binding activity and results in a higher disposition to form paired helical filaments (Alonso et al. 1996; Holtzman et al. 2011). This leads to the breakdown of the microtubule network and consequently to neurofibrillary degeneration (Iqbal and Grundke-Iqbal 2008).

Tau is essential for diagnosing and staging of AD (Arnold et al. 1991; Braak and Braak 1991). During the course of AD, Tau aggregation and NFT formation appear later than amyloid deposition (Galimberti and Scarpini 2012). In contrast to amyloid deposition, the NFT formation correlates with progression of the cognitive deficits (Braak and Braak 1991; Holtzman et al. 2011). In some cases the formation of fibrillary bundles in the entorhinal cortex starts many years before the first symptoms of dementia appear. Subsequently, they spread to the hippocampus and to the neo-cortex (Braak and Braak, 1991). It has been shown that the regional number of NFTs highly correlates with the loss of synapses and the stage of cognitive impairment in AD patients (Masliah et al. 1992).

Moreover, there is a variety of so called "tauopathies" - diseases that characterised by neurodegeneration directly induced by pathological aggregates of tau in different filamentous structures such as paired helical filaments, straight filaments, and neuropil threads in the axons and dendrites. For example, familial missense mutations in the Tau gene cause another form of dementia called frontotemporal dementia with parkinsonism (FTDP-17) (Hutton et al. 1998; Spillantini et al. 1998). Pick's Disease (Probst et al. 1996), cortico- 
basal degeneration (Matsumoto et al. 1996), and dementia pugilistica (Hof et al. 1992) also belong to the group of "tauopathies".

However, in the case of $\mathrm{AD}$, there is evidence from in vitro and in vivo models showing that accumulation of amyloid beta peptides is a starting point of the pathology, and deposition of $A \beta$ consequently initiates the fibrillization of tau (Guo et al. 2006).

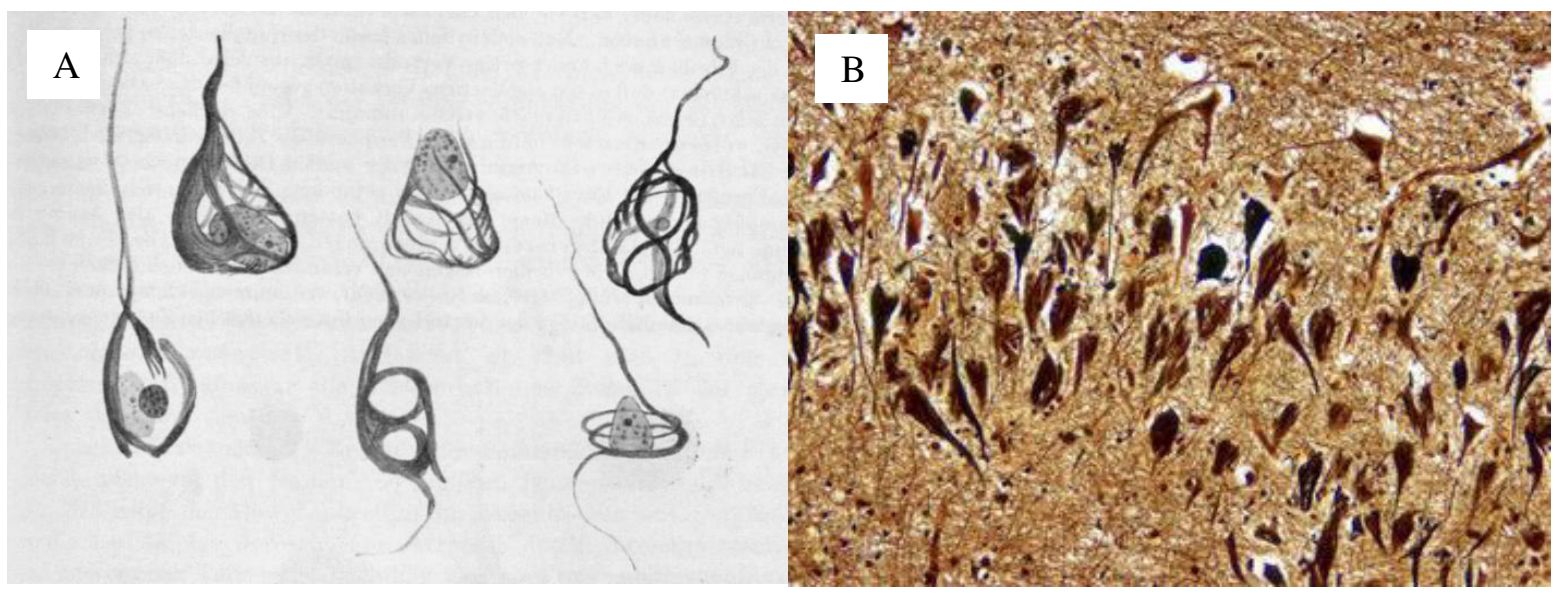

Figure 1. 3: Neurofibrillary tangles. (A) A diagram of the tangles from a 1911 research paper by Alois Alzheimer (Alzheimer 1911). Figure reproduced from http://nyamcenterforhistory.org/. (B) Image of neurofibrillary tangles in cerebral cortex of a patient with Alzheimer's disease visualized with Bielschowsky silver stain. Figure reproduced from www.neuropathology-web.org

\subsubsection{Inflammation}

The role of inflammation in AD brain is not fully understood. Neuroinflammation is consistently found in AD patients (Hoozemans et al. 2006) and also occurs in transgenic models of AD (Benzing et al. 1999; Matsuoka et al. 2001; Dudal et al. 2004). Activated astrocytes and microglia, the complement system as well as cytokines and chemokines are found in the proximity of neuritic plaques. This leads to the conclusion that $\mathrm{A} \beta$ triggers an inflammation process via glia activation (Itagaki et al. 1989; Krause and Müller 2010; Pike et al. 1995b). Following activation, microglia and astrocytes release proinflammatory signal molecules including complement factors, chemokins and cytokines (Rubio-Perez and Morillas-Ruiz 2012; Tuppo and Arias 2005). Several studies suggest that glial cells take up and process A $\beta$ (Koenigsknecht-Talboo et al. 2008; Meyer-Luehmann et al. 2008). However, it remains still unclear whether inflammatory processes are generally harmful and induce neurodegeneration (Hauss-Wegrzyniak et al. 1998). This is just as plausible as understanding them as a protective reaction in an attempt to rescue neurons (Weninger and Yankner 2001). It was suggested that glial cells contribute to the elimination of toxic A $\beta$ species (A $\beta$ clearance), thus, it might be profitable for the treatment of AD (Bard et al. 2000; DeMattos et al. 2012). 


\subsubsection{Brain atrophy and neuron loss}

Atrophic changes and neuron loss are one of the major hallmarks of AD brain, still they have been also found in other dementias such as frontotemporal dementia or vascular dementia (Blennow et al. 2006). The main structures that undergo massive neuronal loss are the entorhinal cortex, the hippocampus (Mizutani et al. 1990), the posterior cingulate cortex, the amygdala, and the temporo-parieto-occipital association cortices (Brun and Englund 1981) but not the inferior frontal and orbitofrontal gyri (Blennow et al. 2006; Duyckaerts et al. 2009; Halliday et al. 2003). These anatomical observations correlate with the psychiatric disorders of patients suffering from AD. In addition, a strong correlation of neuron number and hippocampal/brain volume has been reported (Kril et al. 2004). Furthermore, it has been shown that magnetic-resonance brain imaging can be used to trace the progression from mild cognitive impairment to AD by estimation of hippocampal/brain atrophy (Jack et al. 2005; Jagust 2006). Brain atrophy mainly caused by neuron loss is the most evident macroscopic characteristic of AD (Figure 1.4).

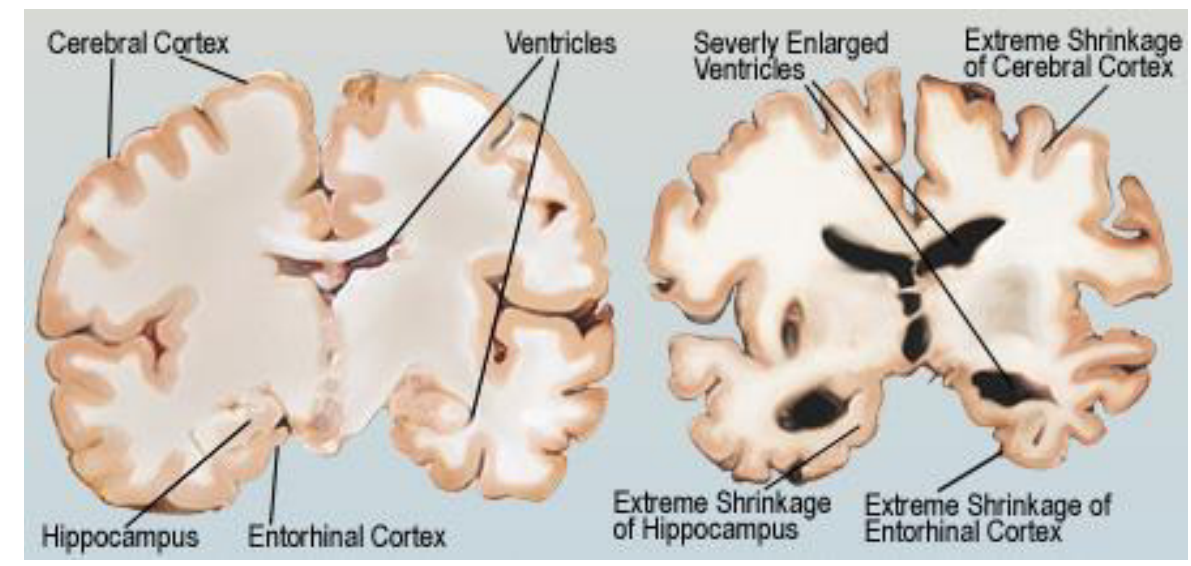

Figure 1. 4: Sagittal sections of brains. Normal adult brain (on the left side) compared with Alzheimer brain (on the right side) showing marked diffuse atrophy in the cortical regions and ventricular enlargement. Figure reproduced from http://memory.ucsf.edu/education/diseases/alzheimer

However, the cause of brain atrophy/neuronal loss remains a subject of ongoing dispute. Most recent reports suggest that intracellular accumulation of aggregated $A \beta$ is crucial for cell death and neuron loss (Bayer and Wirths 2010; Haass and Selkoe 2007; Larson and Lesné 2012). Others reported a correlation of brain region atrophy with the number of NFTs within the same brain region (Cras et al. 1995; Gómez-Isla et al. 1997).

Design based stereology showed a significant neuron loss in the entorhinal cortex of patients with very mild AD (Gómez-Isla et al. 1996). More than $50 \%$ of the neurons in the superior temporal sulcus are lost in patients with AD (Gomez-Ila et al. 1997). A massive neuron loss in the CA1 was reported in AD patients (West et al. 2004), whereas no neuron loss was observed in preclinical AD. Furthermore, brain neuronal loss is observed in various murine models of Alzheimer's disease (Bouter et al. 2014; Casas et al. 2004; Christen- 
sen et al. 2008; Christensen et al. 2010; Jawhar et al. 2012; Meißner et al. 2015; Oakley et al. 2006; Saul et al. 2013; Schmitz et al. 2004; Wirths and Bayer 2010).

Synapse loss is also an early sign of the pathological processes in AD. It has been noticed that the number of lost synapses surpasses the neuron loss in the cortex. It allows assuming that synapse loss starts before neuron loss and contributes to the cortical atrophy (Serrano-Pozo et al. 2011). In addition, decreased synaptic density correlates directly with the cognitive decline in AD patients. According to some studies, synaptic density is considered to be a better correlate of severity of AD compared to NFTs or neuron loss (DeKosky and Scheff 1990; Ingelsson et al. 2004; Scheff and Price 1993; Scheff et al. 1990).

\subsubsection{Alterations in neurogenesis}

Even the adult brain continually generates new neurons. Animal studies showed that the synthetic nucleoside bromodeoxyuridin (BrdU) was incorporated in cells of the subventricular zone (SVZ) and the subgranular zone (SGZ) of the dentate gyrus of the mammalian brain (Kaplan and Hinds 1977). These cells were confirmed to be new-born neurons giving evidence about existence of a mitotic activity in those regions.

Newly generated cells from the SVZ migrate to the olfactory bulb where they differentiate into functional neurons, while new-born cells issued from the SGZ of the dentate gyrus migrate and become integrated in the granule cell layer of the dentate gyrus (Cayre et al. 2009; Crews and Nixon 2003) (Figure 1.5).

In the context of Alzheimer's disease, adult hippocampal neurogenesis attracts huge attention, because it is involved in higher cognitive function, especially memory processes, and certain affective behaviours. Adult hippocampal neurogenesis appears to contribute significantly to hippocampal plasticity during lifetime, because it is regulated by activity. New excitatory granule cells are generated in the dentate gyrus, whose axons form the mossy fibre tract that connects the dentate gyrus to the CA3 region. Neurogenesis starts from a population of radial glia-like precursor cells (type 1 cells) that have astrocytic properties. They express markers of neural stem cells and divide rarely. Type 1 cells give rise to intermediate progenitor cells with first glial (type 2a) and then neuronal (type 2b) phenotype. Going through a migratory neuroblast-like stage (type 3), the new-born cells exit the cell cycle and enter a maturation stage. During maturation stage, they extend their dendrites into the molecular layer and their axon to the CA3 region. During a period of several weeks, the new cells show increased synaptic plasticity, before finally being integrated and becoming indistinguishable from the older granule cells (Kempermann et al. 2015) (Figure 1.6).

It was discovered that endogenous and environmental factors, as well as drugs can modulate the neurogenesis in a positive or negative way (see also 1.8.2). For example, physical activity (van Praag et al. 1999), as well as hormonal levels (Cameron and Gould 1994), diet (Stangl and Thuret 2009), or cannabinoids (Jiang et al. 2005) and antidepressant drugs (Boldrini et al. 2009) can affect the number of new-born neurons (Figure 1.7). 


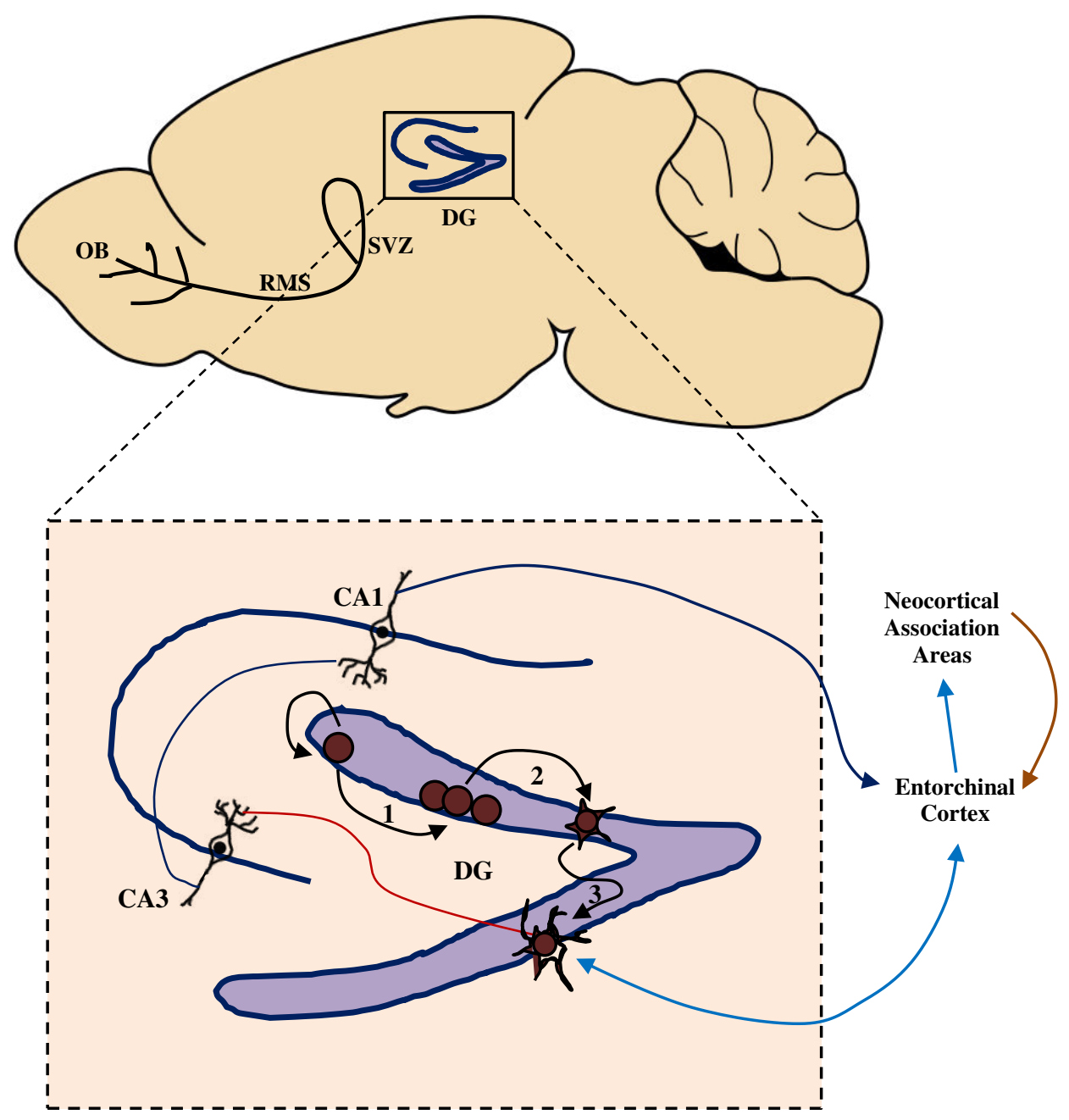

Figure 1. 5: Schematic sagittal view of a rodent brain pointing out the two neurogenic zones of the adult mammalian brain: the subventricular zone (SVZ) of the anterior lateral ventricles and the subgranular zone (SGZ) of the dentate gyrus (DG) in the hippocampus. New-born neurons generated in the SVZ of the anterior lateral ventricles migrate through the rostral migratory stream toward the olfactory bulb where they become functional neurons. Cells dividing in the SGZ of the dentate gyrus become incorporated into the granular cell layer before completely differentiate into granule cells. The enlarged hippocampal region (black square) shows (1) neural progenitor cells in the SGZ of the DG proliferating, (2) migrating into the granule cell layer and (3) maturing into new granule neurons. These integrate into the hippocampal circuitry by receiving inputs from the entorhinal cortex and extend projections into the CA3. Figure created after Stangl and Thuret 2009, p. 273.

However, the role of neurogenesis in memory and learning is still not well understood as a wide range of studies show contradictory results. It was shown that blocking hippocampal neurogenesis results in better performances in hippocampus-dependent memory tasks (Saxe et al. 2007). Other studies showed the opposite effect: the inhibition of neurogenesis decreased learning abilities (Hu et al. 2009; Jessberger et al. 2009). Neurogenesis is also a subject of discussion in the field of Alzheimer research due to controversial findings about the neurogenic behaviour of diseased brains. Indeed, neurogenesis was found to be either increased or decreased both in rodents and humans (Chevallier et al. 2005; Haughey et al. 2002; Taupin 2009; Ziabreva et al. 2006). 


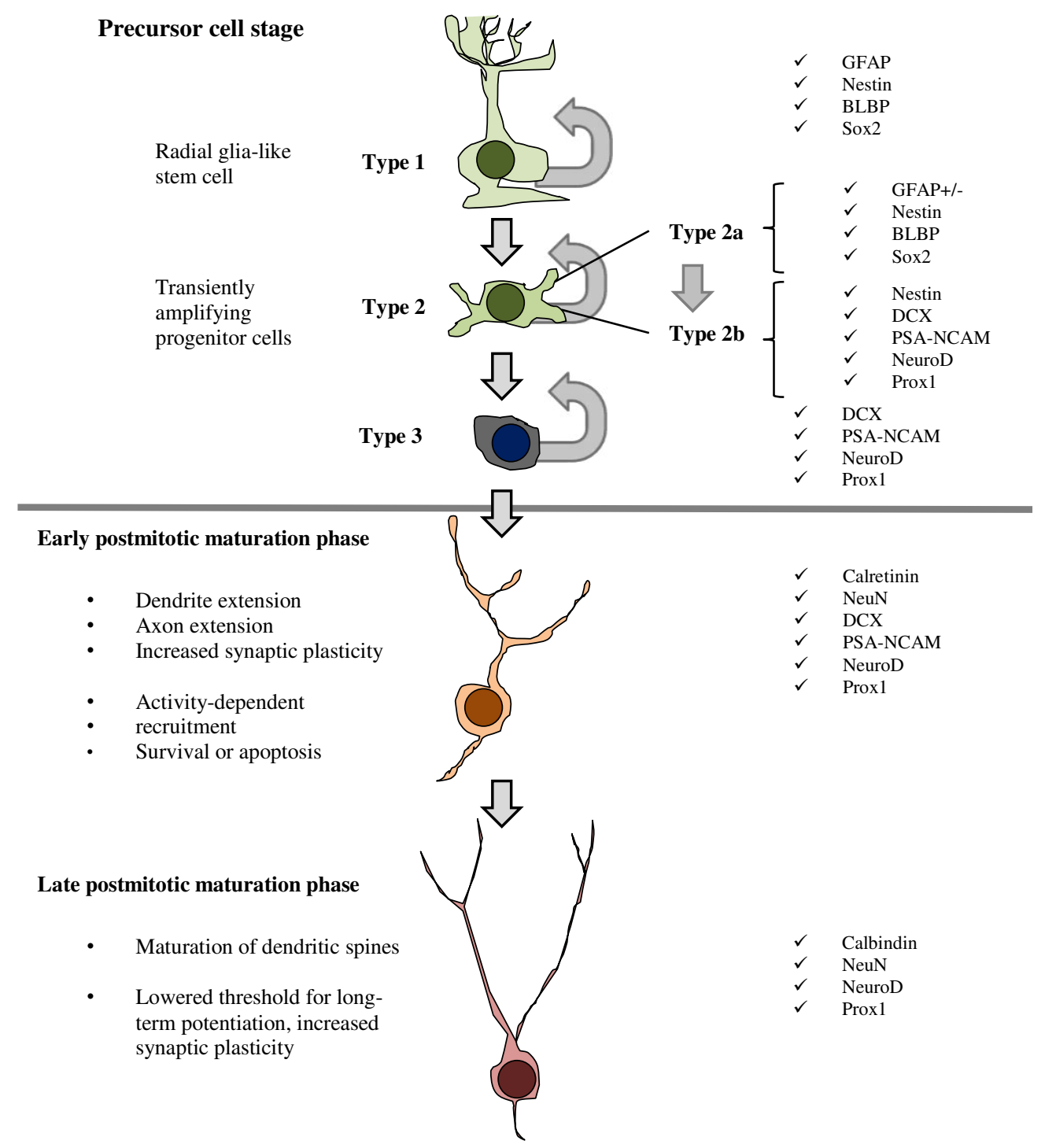

Figure 1. 6: Developmental stages in the course of adult hippocampal neurogenesis (GFAP, Glial fibrillary acidic protein; BLBP, brain lipid-binding protein; DCX, doublecortin; PSA-NCAM, polysialilated neuralcell-adhesion molecule). Figure created after Kempermann et al. 2015, p. 4.

Further facts about adult neurogenesis under other pathological conditions are very interesting as well. Following brain trauma or ischaemia, proliferation is increased in the dentate gyrus and the SVZ (Yagita et al. 2002; Kokaia and Lindvall 2003; Parent 2003). In addition, new-born neurons can also be found in the striatum and neocortex. These latter effects have been ascribed to the atypical migration of SVZ neuronal precursors towards damaged areas. Therefore, pathological conditions might induce endogenous neurogenesis in regions where adult neurogenesis is usually non-existent (Dash et al. 2001; Zhang et al. 2004).

Despite controversial discussion, adult neurogenesis is considered to be a serious therapeutic target in AD treatment, because of the multiple possibilities of its modulation. This topic will be discussed in section 1.8.1. 


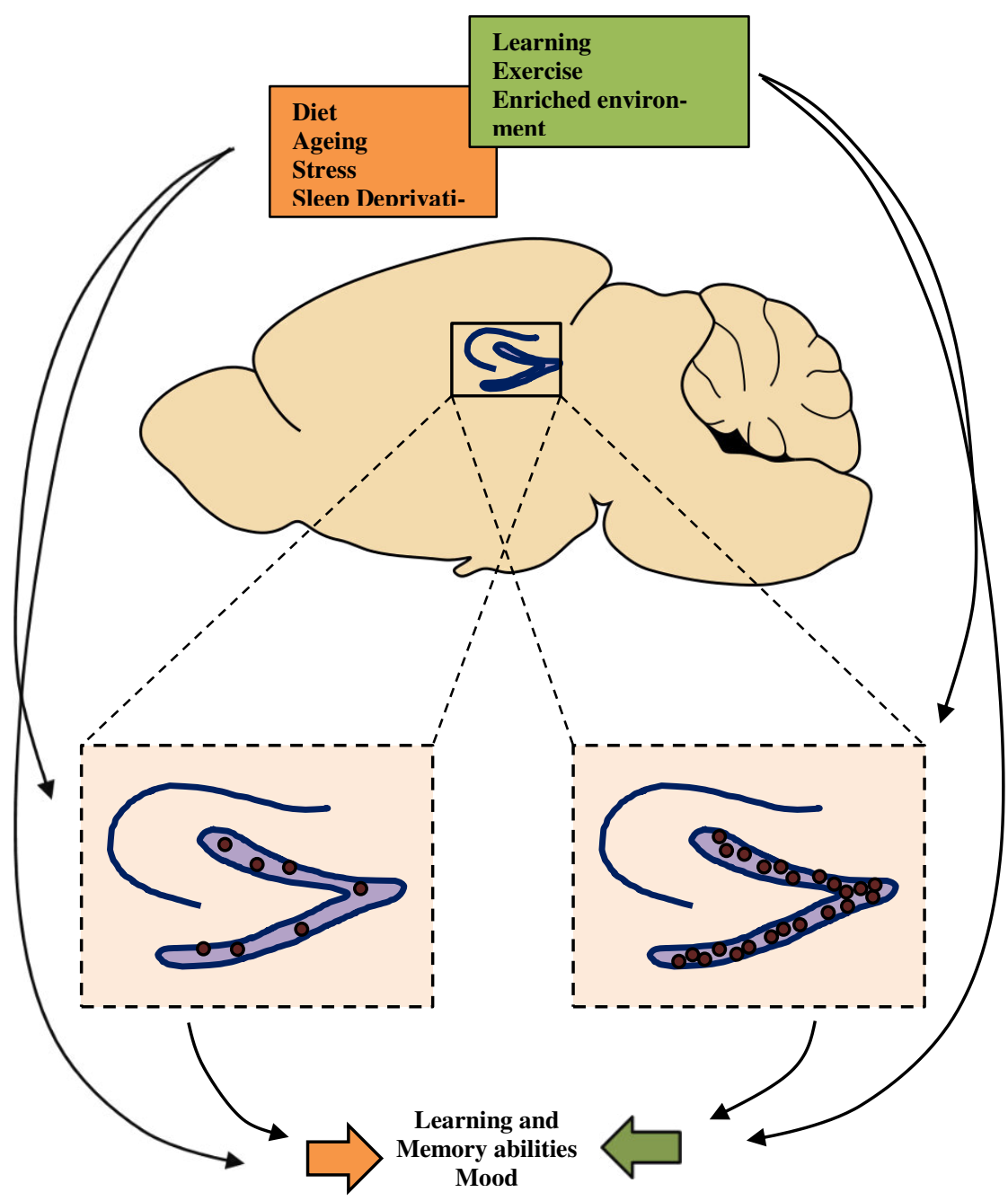

Figure 1. 7: Overview of the physiological and environmental modulation of adult hippocampal neurogenesis and its influence on learning, memory abilities, and mood. In the enlarged hippocampus (dotted squares), the red dots symbolize newborn neurons in the dentate gyrus (DG). Figure created after Strangl and Thuret 2009, p. 276.

\subsubsection{Motor impairment. Axonopathy}

One of the most relevant symptoms at the later stages of $\mathrm{AD}$ is the progressive decline of motor functions.

Along with cognitive decline, motor deficits make patients helpless and dependent even in performing the simplest motor tasks, and burden their caregivers (Flannery 2002). Motor deficits can be so severe that patients are not able to swallow properly and can choke with meal. That can lead to aspiration pneumonia and death (Wada et al. 2001). At the early stages of $\mathrm{AD}$, this symptom manifests mainly in early deficits in balance (Rolland et al. 2009) and motor coordination (Persad et al. 2008; Yan et al. 2008) increasing the risk of falls (Allan et al. 2009). Decline in motor performances is regarded as a potential predictor of AD progression and severity (Hebert et al. 2008) and can help distinguishing between mild cognitive impairment and early stages of AD (Pettersson et al. 2005). Paying attention to the early motor impairments may enable earlier pharmacological or environ- 
mental interventions, which could slow down the course of AD. A range of transgenic mouse models of AD also showed age-dependant motor impairments (Jawhar et al. 2012; Wirths and Bayer 2008; Wirths et al. 2010b). It has been hypothesised that motor deficits can be explained by insufficient cerebral blood flow in the visual area (Nakano et al. 1999) or hyperexcitability of neuronal networks of the motor cortex (Ferreri et al. 2003).

It was also noticed that prominent defects in axonal transport correlate with impairment of motor performance and degenerative processes (Chevalier-Larsen and Holzbaur 2006; Wirths et al. 2007). Thus, impaired axonal transport also may be the cause of motor deficits.

Axonal defects can manifest itself as axonal swellings or spheroids, which correspond to axonal enlargements and aberrant accumulation of axonal cargoes, cytoskeletal proteins and lipids (Wirths et al. 2007) (Figure 1.8). Swollen axons and dendrites with these accumulations are often called dystrophic neurites, and the range of all axonal abnormalities and deficits is called axonopathy.

The observation of abnormal filaments within the cytoskeleton of dystrophic neurites in pre and post mortem brain tissue of $\mathrm{AD}$ patients led to the hypothesis that dysfunctional fast axonal transport could be part of the AD pathology (Praprotnik et al. 1996). Cortical neurons extracted post mortem from temporal cortex of AD patients showed significantly impaired axonal transport in comparison to healthy controls (Dai et al. 2002). Further animal studies showed for instance that in vivo application of $A \beta_{1-42}$ blocks fast axonal transport in rat sciatic nerve (Kasa et al. 2000).

The production of neurotoxic $A \beta$ and the formation of hyperphosphorylated Tau are supposed to be crucial steps contributing to the neuropathological mechanisms in Alzheimer's disease (AD). However, the exact relationship between axonopathy and the origin and development of classic neuropathological changes such as amyloid plaques and NFTs remains unclear (Xiao et al. 2011).

Axonal deficits and impairment of motor functions are common pathological alterations in mouse models expressing different isoforms of human mutant tau protein (Wirths and Bayer 2008).

However, in recent years, similar phenotypes and indications for disorders in axonal transport and axonopathy have been also described in different APP-based transgenic AD mouse models (Adalbert et al. 2009; Jawhar et al. 2012; Salehi et al. 2006; Stokin et al. 2005; Wirths et al. 2006; Wirths et al. 2007) and in APP-based models of Down syndrome (Salehi et al. 2003).

One of the suggested mechanisms of fast axonal transport disruption in AD implied that mutant presenilins induce phosphorylation of the kinesin light chain by GSK3 $\beta$ that disrupts the attachment of kinesin to vesicles. Therefore, the vesicles are not being transported. GSK $3 \beta$ also plays a role in the hyperphosphorylation of Tau. In its hyperphosphorylated form, Tau disorganizes the microtubule structure and interrupts the traffic of vesicles (De Vos et al. 2008). A block of axonal transport can also cause "dying back" axon degeneration (Coleman 2005; Ferri et al. 2003) and cell death (Hafezparast et al. 2003; LaMonte et al. 2002).

Moreover, a scientific debate has raged on the issue whether $A \beta$ acts as a trigger for impaired axonal transport in the pathophysiology of AD (Christensen et al. 2014; Wirths et 
al. 2007). Evidence that axonopathy happens in vivo without Tau hyperphosphorylation (Wirths et al. 2007) indicates that intraneuronal $A \beta$ can also be a potent factor for disrupting axonal transport (Bayer and Wirths 2008; Pigino et al. 2009). However, it is still unclear, whether axons become so severely dystrophic because of their proximity to amyloid plaques, or whether amyloid plaques, which are situated closely to the damaged axons, are partly composed from proteins leaking from the dystrophic neurites. Direct leakage of intra-axonal components, including structural and functional proteins, to extra-axonal space may be involved in the formation of plaques. For instance, the leakage of overproduced intra-axonal $A \beta$ or extra-axonal proteolysis of the leaked APP may result in A $\beta$ plaques (Xiao et al. 2011).

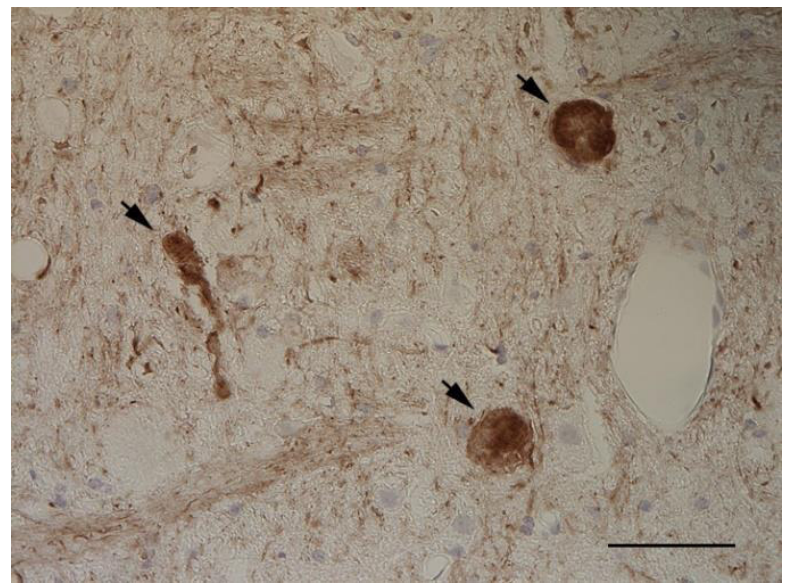

Figure 1. 8: Axonal swellings. Neurofilament-L stained brain section of 5XFAD mouse showing axonopathy (arrows $=$ swollen axons); scale bar: $33 \mu \mathrm{m}$. Source: own research.

\subsection{Pathomechanisms of Alzheimer's disease}

\subsubsection{The Amyloid Precursor Protein}

The amyloid precursor protein (APP) is a type-1 transmembrane glycoprotein, which is highly conserved and is ubiquitously expressed. APP belongs to the amyloid-precursorlike protein family (Puzzo et al. 2014; Selkoe et al. 1988). APP as transmembrane glycoprotein consists of three parts: the intracellular region, the extracellular region, which is much larger than the intracellular one, and the transmembrane domain (Castellani et al. 2009; Kandel et al. 2000).

In humans, the APP gene is located on chromosome 21. A $\beta$ peptides, which are the main components of plaques and which are responsible for $\mathrm{AD}$ and other amyloidosis, are derived from sequential cleavage of APP (Castellani et al. 2009; Korenberg et al. 1989).

Though the members of the amyloid-precursor-like protein family are structurally highly conserved, they show large heterogeneity in the A $\beta$ region (Selkoe 2001). Through alternative splicing and different post-translational modifications, a variety of isoforms of 
APP are expressed in different types of tissues. These isoforms are categorised by the number of amino acid they contain. Among them, $\mathrm{APP}_{695}, \mathrm{APP}_{751}$ and $\mathrm{APP}_{770}$ are the three major forms expressed in neuronal cells (Sandbrink et al. 1996). $\mathrm{APP}_{695}$, is mainly expressed in neurons of the central nervous system. The highest levels of its expression have been detected in the cortex, hippocampus and cerebellum (Solà et al. 1993). APP ${ }_{751}$ and $\mathrm{APP}_{770}$ are also widely expressed in other tissues (O'Brien and Wong 2011; Selkoe 2001).

Several physiological functions have been attributed to APP: synaptogenesis, neurite outgrowth, protein transport along the axons, cell adhesion and calcium metabolism (Zheng and Koo 2006). APP is also thought to be involved in neuronal migration (YoungPearse et al. 2007) and gene transcription involved in cell death (Kögel et al. 2005). In spite of this, the role of APP still remains not well understood. For instance, APP knockout mice are not affected in their vital functions and bear viable offspring. However, APP knockout mice show cerebral gliosis, defects in learning and long term potentiation, and reduced body and brain size (Dawson et al. 1999; Ring et al. 2007; Zheng et al. 1995). It has been suggested that the homologous APP-like proteins may partly perform the functions of APP in knockout mice so that the lethal phenotype can be avoided (Heber et al. 2000; Slunt et al. 1994).

\subsubsection{APP processing}

\subsubsection{Non-amyloidogenic pathway}

APP can be processed via two alternative pathways: the non-amyloidogenic and the amyloidogenic pathway (De-Paula et al. 2012) (Figure 1.9). In the non-amyloidogenic pathway, APP is cleaved within the A $\beta$ region by an $\alpha$-secretase releasing the $\operatorname{sAPP} \alpha$ (a large soluble N-terminal fragment) and a smaller C-terminal fragment C83 (Esch et al. 1990; Sisodia et al. 1990), which is further cleaved by $\boldsymbol{\gamma}$-secretase releasing the soluble Cterminal p3 fragment and the APP intracellular domain (AICD) (Carrillo-Mora et al. 2014; Querfurth and LaFerla 2010). Various enzymes have been suggested to act as $\alpha$-secretase. Among them are three members from the ADAM family (a disintigrin and metalloprotease family): $\mathrm{ADAM}_{9}, \mathrm{ADAM}_{10}$ and $\mathrm{ADAM}_{17}$ (Haass 2004; Haass et al. 2012). Recent studies indicate that $\mathrm{ADAM}_{10}$ functions as the main $\alpha$-secretase in neurons (Kuhn et al. 2010).

After cleavage of $\mathrm{C} 83$ by $\gamma$-secretase, the p3 fragment rapidly degenerates (De-Paula et al. 2012), while AICD is supposed to act as transcriptional regulator (Chow et al. 2010). Because the cleavage of APP by $\alpha$-secretase occurs within the A $\beta$ region, the generation of amyloid peptides is prevented (Sisodia 1992). In contrast to A $\beta$ species, sAPP $\alpha$ has been suggested to have neuroprotective activity (Chow et al. 2010; Furukawa et al. 1996; Mattson 1997). 


\subsubsection{Amyloidogenic pathway}

The amyloidogenic pathway (Figure 1.9) is a mechanism of APP cleavage, which results in release of A $\beta$ (De-Paula et al. 2012). In contrast to the non-amyloidogenic pathway, here APP is first cleaved by a $\beta$-secretase releasing a soluble $\mathrm{N}$-terminal fragment (sAPP $\beta$ ) and a longer $\mathrm{C}$-terminal fragment C99. $\beta$-secretase cleaves the amyloid precursor protein 16 amino acids upwards from the $\alpha$-secretase cleavage site. The membrane-bound C99 fragment is subsequently processed by $\gamma$-secretase. $\gamma$-secretase cleaves C99 within the transmembrane domain at different sites producing $A \beta$ peptides of various lengths and AICD. The length of $A \beta$ peptides range from 37 to 43 aminoacids (Annaert and Strooper 2002; Citron et al. 1995; De-Paula et al. 2012; Vassar et al. 1999; Wolfe 2007; Zhang et al. 2011). Physiologically, $A \beta$ mainly consists of 40 amino acids. Under normal physiological conditions, the 42 amino-acid long $A \beta$, which constitutes amyloid plaques, is only a minor portion of the total $A \beta$ (Zhang et al. 2011).

\section{A Non-amyloidogenic pathway}

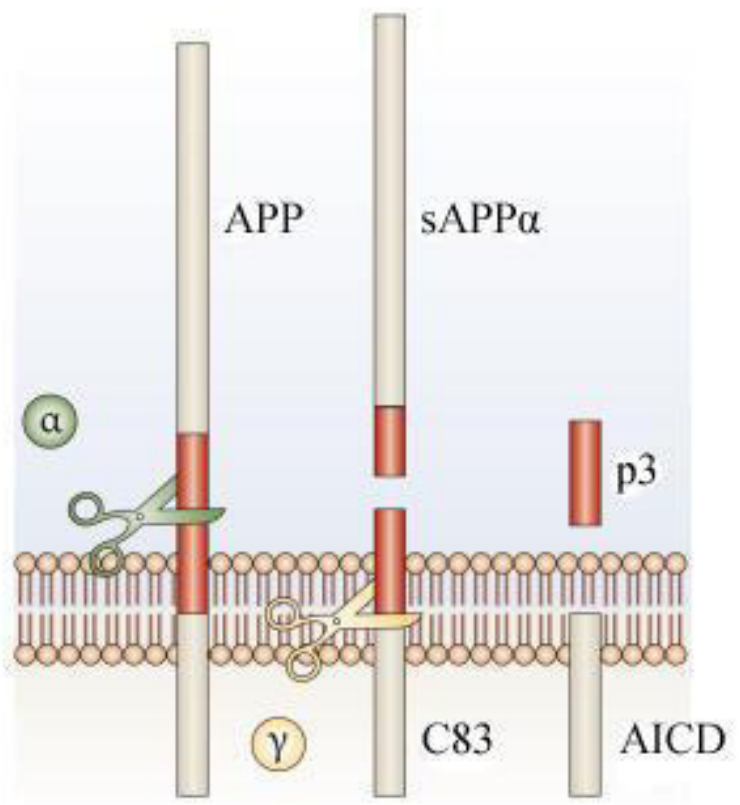

B Amyloidogenic pathway

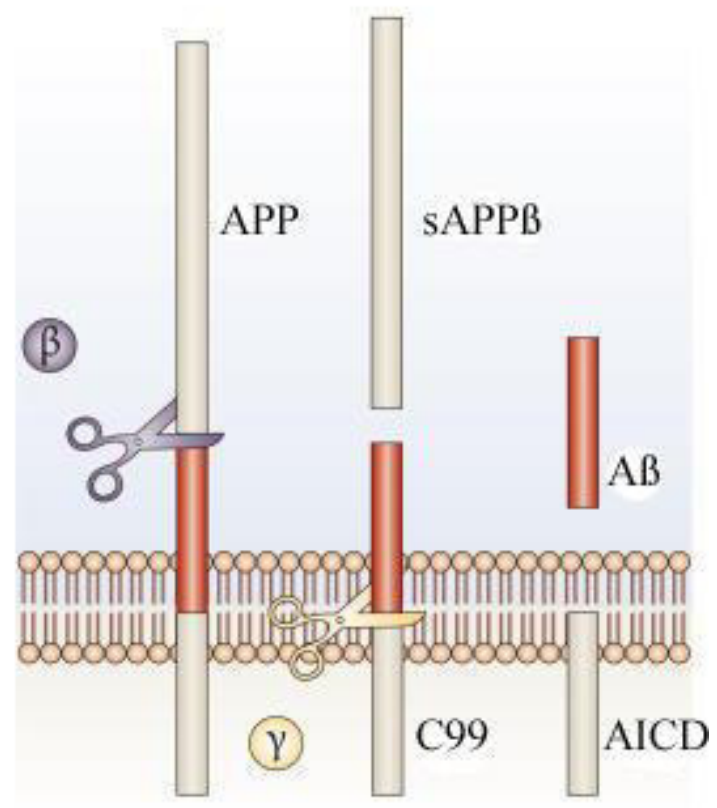

Figure 1. 9: APP processing by secretases. Schematic diagram showing non-amyloidogenic (A) and amyloidogenic pathways (B). (A) In the non-amyloidogenic processing, APP is first cleaved by $\alpha$-secretase within the A $\beta$ sequence releasing sAPP $\alpha$. Then, the resulting carboxy-terminal fragment is processed by $\gamma$-secretase releasing the $\mathrm{p} 3$ fragment and the APP intracellular domain (AICD). No A $\beta$ is produced. (B) The amyloidogenic pathway is initiated when $\beta$-secretase (BACE1) cleaves APP at the amino terminus of the A $\beta$ peptide and releases SAPP $\beta$. Further cleavage of the resulting carboxyl terminal by $\gamma$-secretase results in the release of A $\beta$ and AICD. Abbreviations: A $\beta$, amyloid- $\beta$; APP, amyloid precursor protein; sAPP $\alpha$, soluble amyloid precursor protein- $\alpha$; sAPP $\beta$, soluble amyloid precursor protein- $\beta$; AICD, APP intracellular domain; C83, carboxy-terminal fragment 83; C99, carboxy-terminal fragment 99. Figure modified from De Strooper et al. 2010, p. 101.

Various enzymes are acting as $\boldsymbol{\beta}$-secretase. The $\beta$-site cleaving enzyme 1 (BACE1) was identified as the primary $\beta$-secretase (Hussain et al. 1999; Sinha et al. 1999; Vassar et 
al. 1999; Yan et al. 1999). BACE1 is a membrane-bound aspartyl protease and can cleave $\mathrm{A} \beta$ at two known positions: the aspartate at position 1 and the glutamate at position 11 (Sinha et al. 1999; Vassar et al. 1999). BACE1 is highly expressed in the brain, particularly in regions affected by AD (Yang et al. 2003; Vassar et al. 1999; Johnston et al. 2005). BACE2 and cathepsin B have been suggested as additional $\beta$-secretases (Hook et al. 2005; Hook et al. 2009). The physiological contribution of these enzymes to APP processing requires further investigation

The $\boldsymbol{\gamma}$-secretase involved in the amyloidogenic pathway, is a complex protease composed of several components: the membrane-bound proteins presenilin 1 or presenilin 2 , nicastrin, anterior pharynx defective-1, presenilin enhancer protein-2, and the cluster of differentiation 147 (Kaether et al. 2006; O'Brien and Wong 2011; Zhou et al. 2006).

Several studies have indicated that upregulation of $\alpha$-secretase activity leads to a lower production of A $\beta$ in subcellular compartments (Nitsch et al. 1992; Postina et al. 2004).

\subsubsection{The amyloid cascade hypothesis}

\subsubsection{The classic amyloid cascade hypothesis}

In the early 90s, the amyloid cascade hypothesis has been proposed by Hardy and Higgins (1992) to explain the etiology and pathogenesis of Alzheimer's disease (Hardy and Higgins 1992). According to this hypothesis, the deposition of $A \beta$ peptides is the cause of all the neurodegenerative processes found in SAD and FAD such as neurofibrillary tangles, synaptic deficits, neuron loss, inflammation, vascular damage, and cognitive decline (Duyckaerts et al. 2009; Hardy and Allsop 1991; Selkoe 1991) (Figure 1.10). The hypothesis states that increased $A \beta$ production or decreased $A \beta$ clearance result in the accumulation of hydrophobic $A \beta_{40}$ and $A \beta_{42}$ and subsequent formation of insoluble extracellular $A \beta$ plaques. Further, $A \beta$ plaques trigger a cascade of changes resulting in dementia and all of neuropathological alterations mentioned above (Hardy and Allsop 1991; Pimplikar 2009).

The amyloid cascade hypothesis has been supported by the following findings:

(1) Numerous familial forms of $\mathrm{AD}$ were characterized, in which the inherited mutations in APP, PSEN1 and PSEN2 alter the A $\beta$ levels with a significant increase in the $A \beta_{1-42} / A \beta_{1-40}$ ratio and invariably lead to increased depositions of $A \beta$ in plaques (Bertram et al. 2010; Murayama et al. 1999; Pimplikar 2009; Rossor et al. 1993).

(2) Many AD mouse models with familial AD mutations also develop similar agedependant pathologies such as $\mathrm{A} \beta$ plaques, gliosis and cognitive deficits (Duyckaerts et al. 2008; Pimplikar 2009). 
(3) Down syndrome patients, which are carriers of an additional chromosome 21 and consequently an additional copy of the APP gene, also develop AD pathology with amyloid plaques and NFTs (Rumble et al. 1989; Schupf and Sergievsky 2002).

(4) $\varepsilon 4$ allele of the Apolipoprotein E gene, which are considered to be the most important risk factor for late-onset $\mathrm{AD}$, is associated with increased $\mathrm{A} \beta$ deposition and reduced A $\beta$ clearance (see 1.3.3) (Bickeböller et al. 1997; Castellano et al. 2011).

Several other observations strengthened the theory of Hardy and Higgins. For example, mutations in the Tau gene lead to other tauopathies with clinical phenotypes different from AD (e.g. frontotemporal dementia). Moreover, it has been shown that formation of NFTs in a transgenic mouse model with P301L Tau mutation was directly triggered after the injection of $A \beta$ fibrils (Götz et al. 2001). Therefore, it has been suggested that NFTs cannot be the initial event in AD course but are likely to occur subsequent to $\mathrm{A} \beta$ deposition (Goedert and Jakes 2005; Hutton et al. 1998; Iqbal et al. 2005).

However, it remains unclear, if the amyloid deposition is the crucial event in the development of $\mathrm{AD}$, why it does not correlate with the severity of dementia neither in humans (Price and Morris 1999) nor in transgenic animal models (Moechars et al. 1999; Schmitz et al. 2004). Robust plaque pathology could be observed in otherwise cognitively normal individuals with no signs of dementia (Pimplikar 2009; Snowdon 2003; Villemagne et al. 2008). It has been shown that plaques in human patients are present years before memory deficits appear and that plaque deposition is almost at maximal levels by the time of diagnosis (Jack et al. 2010; Morris and Price 2001; Price et al. 2009). This discovery indicates that further plaque deposition is not connected to the progression of the disease. Moreover, in contrast to human $\mathrm{AD}$ patients, in many $\mathrm{AD}$ mouse models memory deficits and other pathological alterations occur long before plaques can be detected (Lesné et al. 2008; Walsh and Selkoe 2007).

\subsubsection{Intracellular amyloid hypothesis}

The first report on intracellular $A \beta$ was published in Masters et al. (1985). Later it was shown, that $A \beta$ can be found intracellularly in pyramidal neurons of the hippocampus, entorhinal cortex prior to formation of plaques and NFTs (Fernández-Vizarra et al. 2004; Gouras et al. 2000). Among these discoveries, intraneuronal A $\beta$ has been also found in young patients with Down syndrome without showing amyloid plaques (Gyure et al. 2001; Mori et al. 1992). Further, it has been shown that decreased intracellular A $\beta$ is accompanied by increased plaque pathology and may therefore contribute to the development of extracellular plaques (Gyure et al. 2001; Gouras et al. 2010). The fact that intracellular A $\beta$ aggregation precedes the formation of plaques and NFTs indicates that it could be one of the earliest pathological events in the AD brain (Wirths and Bayer 2012; Wirths et al. 2004).

With the growing evidence that intraneuronal $A \beta$ may be a key contributor in the development of $\mathrm{AD}$, the amyloid cascade hypothesis was modified and intracellular $\boldsymbol{A} \boldsymbol{\beta} \boldsymbol{a c}$ cumulation was suggested as the triggering event of neurodegenerative processes in the 
brain (Haass and Selkoe 2007; Wirths et al. 2004) (Figure 1.10). The authors suggest that intraneuronal $A \beta$ can originate from intra- and extracellular space. Besides intracellular cleavage of APP, the reuptake of A $\beta$ from the extracellular space may be the second mechanism. In this case, amyloid plaques might serve as a reservoir for neurotoxic A $\beta$ oligomers, which can affect synaptic structure and plasticity (Wirths et al. 2004).

Several receptors, which may enable the reuptake of $A \beta$, have been suggested. Among them, there are Apolipoprotein E receptors, nicotinic acetylcholine (nAChR) receptors, integrins and N-methyl-D-aspartate (NMDA) receptors as well as advanced glycation end product receptors.

Accumulation of $A \beta_{42}$ inside neurons has a variety of severe consequences (Mohamed and Posse de Chaves 2011). Intraneuronal A $\beta$ is believed to be responsible for the cell functional failure through mitochondrial arrest (Rhein et al. 2009), following by neuroinflammation and oxidative stress (Mohandas et al. 2009). In vitro experiments showed that in neuronal cell culture intracellular $\mathrm{A} \beta$ can be localized in endosomes, lysosomes and multivesicular bodies almeida(Almeida et al. 2006; Mohamed and Posse de Chaves 2011; Runz et al. 2002; Takahashi et al. 2004). Accumulation of A $\beta$ in lysosomes causes the loss of lysosomal impermeability and leads to leakage of lysosomal contents such as proteases and cathepsins resulting in apoptosis and necrosis (Ditaranto et al. 2001; Liu et al. 2010; Nixon 2005). It was also shown that intraneuronal oligomeric A $\beta$ species are involved in the disruption of fast axonal transport (Pigino et al. 2009).

The important role of intracellular $\mathrm{A} \beta$ in $\mathrm{AD}$ was confirmed in a variety of animal models (Bayer and Wirths 2008; Casas et al. 2004; Christensen et al. 2008; Jawhar et al. 2012; Oddo et al. 2003; Shie et al. 2003; Wirths et al. 2001). The toxicity of intracellular $\mathrm{A} \beta$ was demonstrated in in vitro studies: in cultured rat cortical neurons, apoptosis was triggered by the presence of $A \beta_{1-42}$ inside of the cells (Kienlen-Campard et al. 2002). The correlation between intraneuronal $A \beta$ and neuron loss reinforced the modified amyloid hypothesis (Casas et al. 2004; Christensen et al. 2008; Jawhar et al. 2012). The relevance of intracellular $\mathrm{A} \beta$ has been demonstrated in mouse models of $\mathrm{AD}$ that show very little or no extracellular amyloid deposition but behavioral deficits and neuron loss in hippocampus (Bouter et al. 2013a; Bouter et al. 2014; Wittnam et al. 2012). Other mouse models, which develop plaques, showed also strong neuron loss in regions where $\mathrm{A} \beta$ accumulates intracellularly (Christensen et al. 2010; Jawhar et al. 2012; Oakley et al. 2006).

Taken together, all studies mentioned above indicate a key role of intracellular $A \beta$ rather than extracellular $\mathrm{A} \beta$ depositions in the etiology and pathogenesis of AD. 


\section{The classic ß-Amyloid cascade}

\section{The modified ß-Amyloid cascade}

Risk factors: aging, Trisomy 21, APP-, PSEN1-, PSEN2-mutations

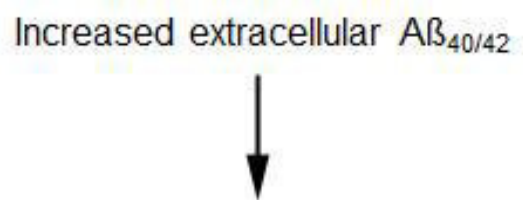

Extracellular $A B_{40 / 42}$ deposits (plaques)
Increased levels of intraneuronal $A B_{40 / 42}$

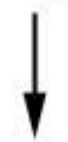

Accumulation of intraneuronal

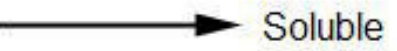
extracellular

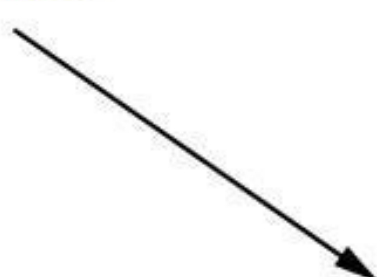

$A B_{40 / 42}$
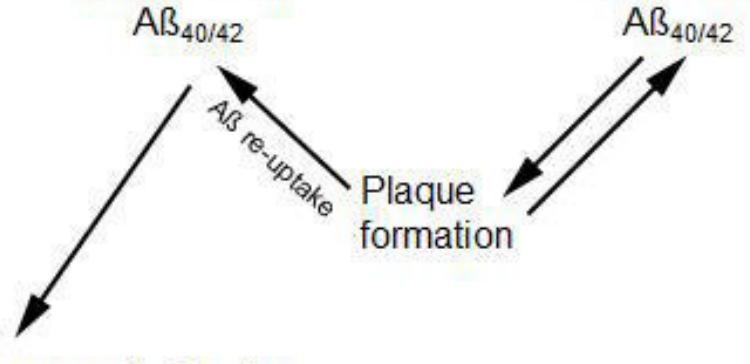

Synapse and neuron dysfunction<smiles>[13CH3]</smiles>

Synapse and neuron loss<smiles>[3H][13CH3]</smiles>

Atrophy of distinct brain areas

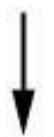

Dementia and other clinical symptoms

Figure 1. 10: Representation of the classic (on the left side) and the modified (on the right side) amyloid cascade hypothesis. The classic amyloid cascade hypothesis states that extracellular deposition of $A \beta$ is the main event in the pathogenesis of $A D$. In contrast, according to the modified intracellular amyloid hypothesis, the intraneuronal accumulation of $\mathrm{A} \beta$ is the crucial event triggering pathological events in Alzheimer's disease. Figure modified after Wirths et al. 2004 , p. 515 , p. 517. 


\subsubsection{Amyloid-beta aggregation}

Soluble $\mathrm{A} \beta$ monomers produced via amyloidogenic pathway have a high propensity to polymerize and aggregate, forming insoluble $A \beta$ fibrils and later plaques. Monomeric A $\beta$ can form dimers, trimers and higher level homo-oligomers (Benilova et al. 2012). The majority of $A \beta$ appears in forms from dimers to octamers. Soluble oligomers possess a nonfibrillar structure that remains stable in aqueous buffers even after high speed centrifugation (Walsh and Selkoe, 2007). At some point, when a certain threshold is reached, oligomers start to polymerise. They self-associate into protofibrils and later to mature fibrils, which are the major component of plaques. Protofibrils can also dissociate back into lower level species (Roychaudhuri et al. 2009; Walsh and Selkoe 2007) (Figure 1.11). Not all A $\beta$ species show the same biochemical properties and therefore have different tendencies to form fibrils. For instance, $A \beta_{1-42}$ has a very high propensity to aggregate in vitro and in vivo (Jarrett et al. 1993). Increased formation and aggregation of neurotoxic $\mathrm{A} \beta$ oligomers have been reported to be a critical step in the initiation and development of AD (Haass and Selkoe 2007; Kayed et al. 2003; Li et al. 2009).

The main neurotoxic properties were first attributed to the insoluble deposits (Walsh and Selkoe 2007). However, the toxicity of extracellular insoluble $A \beta$ forms has been questioned since it has been discovered that brains of cognitively and physically intact older people also can display loads of amyloid plaques (Snowdon 1997). It was demonstrated that soluble oligomeric $A \beta_{42}$ correlates better than plaques with the synaptic loss and cognitive decline in AD patients (Bayer and Wirths 2010; McLean et al. 1999). The higher level of $A \beta$ oligomers has been also found to correlate with synaptic dysfunction (Lue et al. 1999; McLean et al. 1999) and with impairment of long-term potentiation (Walsh et al. 2002). $A \beta$ can aggregate and form oligomers in vitro due to its amphiphilic structure (Pimplikar 2009). Also it is possible to isolate $A \beta$ oligomers from $A D$ brain extracts and from mice brains of transgenic mouse models (Lesné et al. 2008; Shankar et al. 2008).

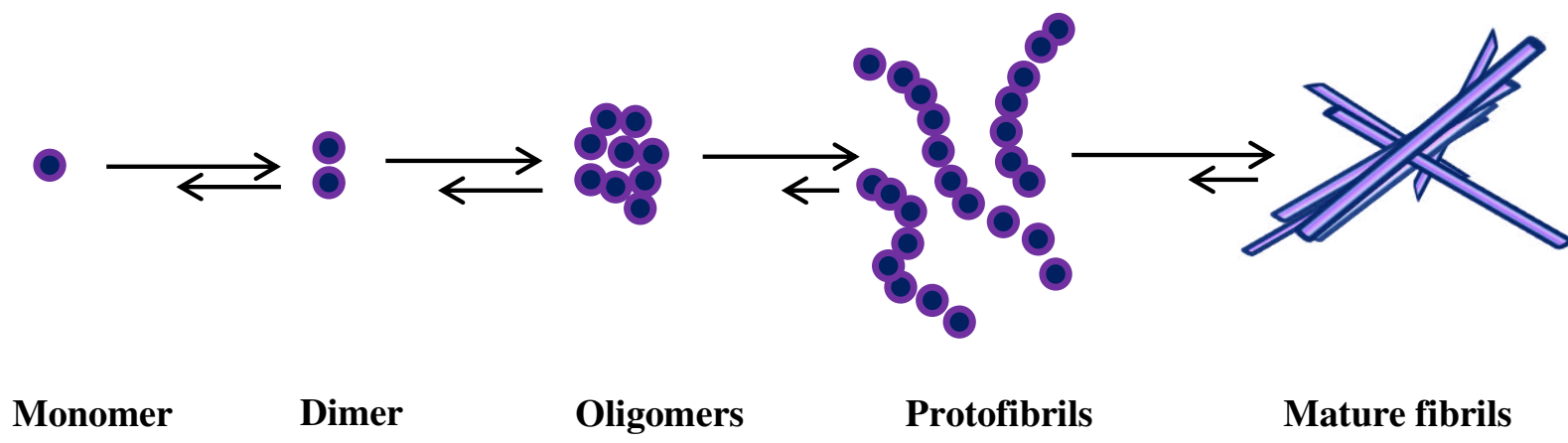

Figure 1. 11: Schematic diagram of $A \beta$ aggregation. Soluble $A \beta$ monomers first form dimers, then oligomers, which subsequently aggregate to protofibrils. Protofibrils assemble to mature amyloid fibrils, which are the main component of plaques. Figure created after Kumar et al. 2011, p. 2263. 


\subsubsection{Amyloid-beta isoforms}

$\mathrm{A} \beta$ peptides observed in the human brain are a heterogeneous set of peptides. A $\beta$ isoforms with varying terminal end lengths differ in aggregation propensity, oligomer stability and structure, resistance to proteolytic degradation, and neurotoxicity (Bouter et al. 2013a; Jan et al. 2008; Jarrett et al. 1993; Pike et al. 1995a; Russo et al. 2002; Wirths et al. 2010a).

The two major $A \beta$ forms are $A \beta_{1-40}$ and $A \beta_{1-42}$. It was shown that $A \beta_{1-40}$ is abundant in cerebral vascular amyloid deposits, while $A \beta_{1-42}$ is the major component of neuritic plaques (Iwatsubo et al. 1994; Suzuki et al. 1994). $A \beta_{1-42}$ is more hydrophobic and has a higher propensity to aggregate than $A \beta_{1-40}$. A $\beta_{1-42}$ is considered more neurotoxic and essential for plaque formation (Jarrett et al. 1993; Jan et al. 2008).

Besides $A \beta_{1-40}$ and $A \beta_{1-42}$, there is a variety of $C$ - and N-terminally truncated $A \beta$ forms. These are for instance $A \beta_{1-15 / 16}$ and $A_{\beta 1-37 / 38 / 39}$ (Portelius et al. 2012; Wiltfang et al. 2002) or a range of longer $A \beta$ variants such as $A \beta_{43}, A \beta_{45}, A \beta_{46}$ and $A \beta_{48}$, that have been identified in cell lines and have also been found in AD mouse models (Esh et al. 2005; Van Vickle et al. 2008; Welander et al. 2009). A $\beta_{43}$ was detected in plaques of human AD patients in at least the same high amount as $A \beta_{40}$ (Welander et al. 2009).

Furthermore, there are various post-translational modifications of $\mathrm{A} \beta$ (Kummer and Heneka 2014): pyroglutamylation, phosphorylation and oxidation, as well as nitration, racemization, isomerization and glycosylation. The most important modification of $A \beta$ is probably the formation of pyroglutamate (pGlu)-modified A $\beta$ species. pGlu modifications of $\mathrm{A} \beta$ lead to its increased aggregation tendency, disturbed proteolytic degradation and exerts high neurotoxicity (Kummer and Heneka 2014; Kuo et al. 1997; Pike et al. 1995a; Russo et al. 2002; Wirths et al. 2010c). Mori et al. (1992) reported that approximately 15$20 \%$ of all $\mathrm{A} \beta$ peptides are N-terminally pyroglutamate-modified. Oxidation and nitration of $\mathrm{A} \beta$ happen as a response to inflammation in $\mathrm{AD}$ (Heneka et al. 2010) and is supposed to prevent the formation of protofibrils (Hou et al. 2002).

\subsubsection{N-terminally truncated $A \beta$}

A variety of $\mathrm{N}$-terminally truncated $\mathrm{A} \beta$ isoforms has been described (Bayer and Wirths 2014; Masters et al. 1985; Mori et al. 1992; Selkoe et al. 1986; Sergeant et al. 2003). Their ragged N-termini result from different cleavage. It has been shown in in vitro experiments that shortening of the $\mathrm{N}$-terminus increases $\mathrm{A} \beta$ aggregation (Pike et al. 1995a) which probably promotes plaque formation in vivo (Soto et al. 1995). Usually, A $\beta$ is cleaved by BASE1 resulting in a peptide with the aspartate at the first position (Asp-1). BASE1 is also able to cleave A $\beta$ between Tyr-10 and Glu-11 leading to production of $A \beta_{11-x}$ species (Vassar et al. 1999). Several proteases have been suggested to be involved in the production of $\mathrm{N}$-truncated $\mathrm{A} \beta$ isoforms (Figure 1.12). However, the exact enzymatic activities mostly remain not well understood (Bayer and Wirths 2014). 
It was demonstrated that the metalloprotease Meprin- $\beta$ is involved in the $A \beta$ cleavage, generating $A \beta$ starting at residue 2 (Bien et al. 2012). A zinc-metalloprotease Neprilysin (neutral endopeptidase or NEP) cleaves A $\beta$ between Arg-2 and Glu-3, Glu-3 and Phe-4, Arg-5 and His-6 (Howell et al. 1995; Iwata et al. 2001; Bayer and Wirths 2014). In addition, it has been reported that myelin basic protein is also able to cleave $A \beta$ between Phe- 4 and Arg-5 at the N-terminus of the A $\beta$-sequence (Liao et al. 2009) as well as plasmin, which produces A $\beta$ species starting at His-6 (Van Nostrand and Porter 1999; Tucker et al. 2000).

$\mathrm{A} \beta$ isoforms with ragged $\mathrm{N}$-termini are highly abundant in the human AD brain (Kawarabayashi et al. 2001; Portelius et al. 2010; Saido et al. 1995). For instance, $A \beta_{2-\mathrm{x}}$ variants are elevated in brains and reduced in the cerebral spinal fluid of AD patients. It is likely that $A \beta_{2-x}$ are a result of cleavage first by BASE1 and then by aminopeptidase A (Bibl et al. 2012; Wiltfang et al. 2001).

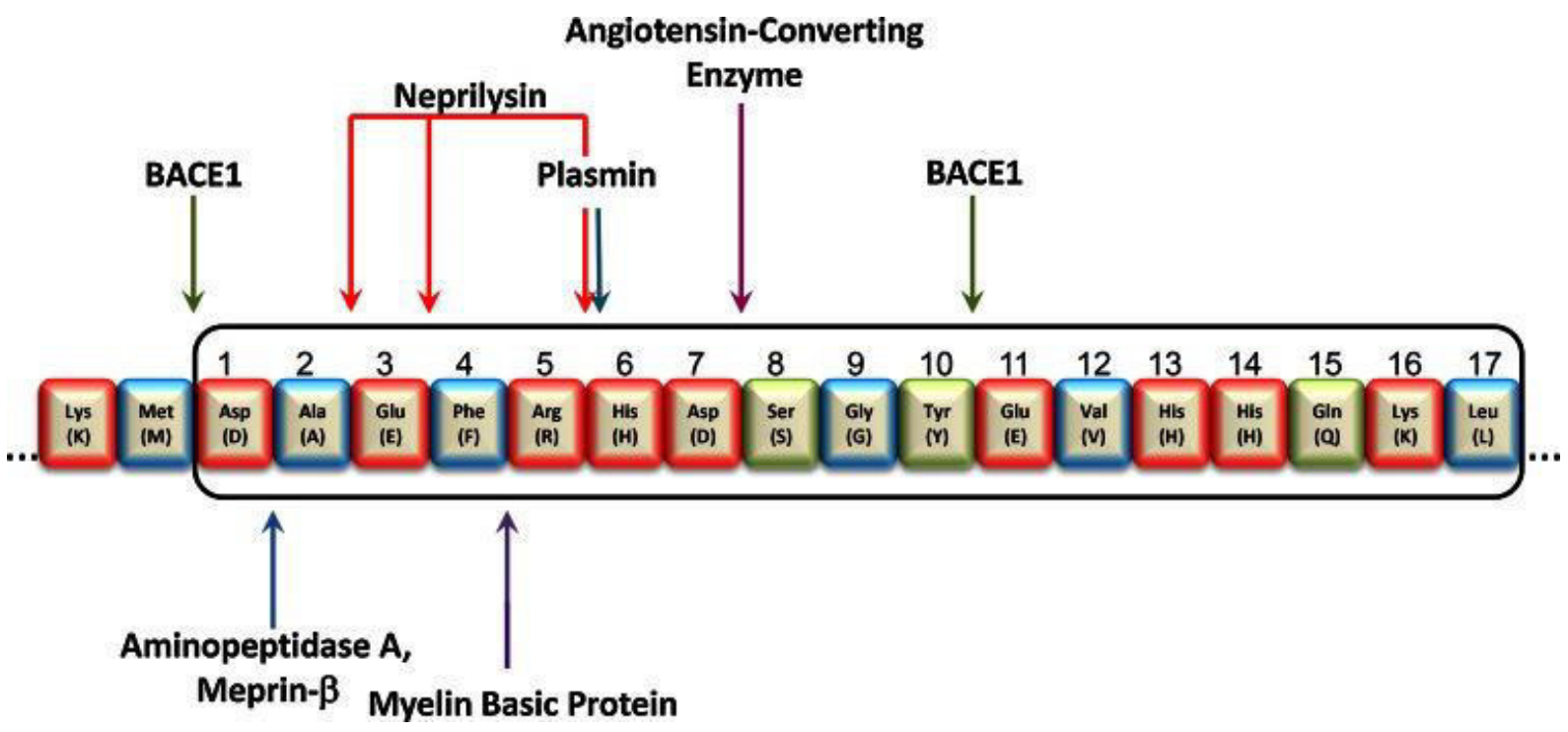

Figure 1. 12: The cleavage sites of enzymes involved in the degradation of full-length $A \beta$ and potential generation of N-truncated $A \beta$ isoforms. Sequence of the first 17 amino acids of the N-terminus of human A $\beta$ is displayed in three-letter and one-letter-code. Colour code for amino acids: red indicates amino acids with charged polar side-chains; green - with uncharged polar side-chains; blue - hydrophobic non-polar amino acids. Figure from Bayer and Wirths 2014, p.789.

$\mathrm{A} \beta_{3-\mathrm{x}}$ has been observed in plaques in late-onset $\mathrm{AD}$ patients and $\mathrm{AD}$ mouse models (Casas et al. 2004; Güntert et al. 2006). It has been shown in vitro that $A \beta$ starting with a pyroglutamate-modified residue three (Glu-3), $\mathrm{A} \beta_{\mathrm{pE} 3-\mathrm{x}}$, oligomerizes more quickly and forms fibrillar, $\beta$-sheet structures more rapidly than $A \beta_{1-x}$ (Harigaya et al. 2000; He and Barrow 1999; Schlenzig et al. 2009). $\mathrm{A} \beta_{\mathrm{pE} 3}$ was found in amyloid plaques in the same amounts as $A \beta_{1-\mathrm{x}}$ or even greater. It has been suggested that $\mathrm{A} \beta_{\mathrm{pE} 3}$ accumulation precedes that of other $A \beta$ species (Saido et al. 1995).

$A \beta_{4-x}$ species are one of the first $A \beta$ discovered. They are abundant in $\mathrm{AD}$ and vascular dementia (Masters et al. 1985; Lewis et al. 2006) (see 1.6.5.2). 
Comparing to $A \beta_{4-x}$ variants, the knowledge about $A \beta_{5-x}$ is very poor. It was detected both in AD patients and non-demented controls (Murayama et al. 2007; Sergeant et al. 2003) The role of $A \beta_{5-x}$ remains still unclear (Bayer and Wirths, 2014).

Apart from $A \beta_{11-x}$, the largest known amino-truncations are cleavages at Asp-7, Ser-8 and Gly-9. Angiotensin-converting enzyme has been suggested as an enzyme leading to the generation of $A \beta_{8-x}$ (Kalback et al. 2002; Kummer and Heneka 2014; Sergeant et al. 2003).

$\mathrm{N}$-truncated $\mathrm{A} \beta$ species are the main components of amyloid plaques in $\mathrm{AD}$ and can also be found intracellularly (Bayer and Wirths 2014; Gouras et al. 2000).

\subsubsection{Amyloid beta 4-42}

$\mathrm{A} \beta$ starting at residue four (Phe-4) is abundant in AD. It was discovered in 1985 as a component of brain amyloid deposits (Masters et al. 1985). However, it is still a not well investigated $A \beta$ isoform. There is not enough knowledge about the contribution of $A \beta_{4-42}$ to the development and progression of AD. After investigation of amyloid depositions in two sporadic AD cases, Masters et al. (1985) reported that $64 \%$ of A $\beta$ peptides from amyloid plaques started with a Phe-4, while, according to (Miller et al. 1993) and (Näslund et al. 1994), $A \beta_{4-42}$ is less abundant in $A D$ brains. Another finding supports the results from Masters et al. (1985) showing that $A \beta_{4-42}$ is one of the major fractions in hippocampus and cortex of human AD patients (Portelius et al. 2010). A $\beta 4-42$ was found in cotton wool plaques in FAD patients with a V261I PSEN1 mutation (Miravalle et al. 2005) as well as in the amyloid deposits in patients with familial Danish dementia and vascular dementia (Tomidokoro et al. 2005; Lewis et al. 2006).

In a recent study from our group, it was shown that $A \beta_{4-42}$ is as toxic as $A \beta_{\mathrm{pE} 3-42}$ and $A \beta_{1-42}$. Moreover, treatment of wild-type mice with intraventricular $A \beta_{4-42}$ injection caused significant working memory deficits. These deficits were similar to the memory impairments induced through $A \beta_{\mathrm{pE} 3-42}$ and $A \beta_{1-42}$ species (Bouter et al. 2013a). It was also demonstrated that $A \beta_{4-42}$ aggregates to oligomers more rapidly than longer $N$-truncated $A \beta$ variants (Pike et al. 1995a) and shows a strong fibrillization propensity (Haupt et al. 2012).

All these studies indicate that $A \beta_{4-42}$ plays an important role in the development and progression of $\mathrm{AD}$.

\subsection{Mouse models of Alzheimer's disease}

\subsubsection{Mouse models and their relevance in AD research}

After the discovery of mutations that lead to familial Alzheimer's disease, a variety of transgenic mouse models has been generated. The majority of these models are based on the overexpression of human APP and/or PSEN1/2 with at least one familial AD mutation. 
The neuropathological alterations, cognitive and motor impairments in these models resemble main characteristics of human AD to a greater or lesser extent. Transgenic animal models differ from each other in their phenotype. That can be attributed to the genetic background, different mutations, different promotors and the transgene doses (Elder et al. 2010). The first successful AD transgenic mouse model based on mutant APP was the PDAPP model. It was reported by Games et al. (1995) and contained human APP with the Indiana mutation $(\mathrm{V} 717 \mathrm{~F})$ under the control of the platelet derived growth factor promotor. PDAPP mice exhibit age-dependant extracellular plaque pathology, dystrophic neurites, gliosis, synapse loss and age-related learning impairment without neuron loss (Chen et al. 2000; Dodart et al. 2000; Games et al. 1995; Irizarry et al. 1997; Reilly et al. 2003)

Another murine model based on the APP mutation is the Tg2576 model. These transgenic mice overexpress human APP with the Swedish mutation (K670N/M671L) under the control of a hamster prion protein promotor (Hsiao et al. 1996). It is one of the most widely studied mouse models of $\mathrm{AD}$, which displays age-dependant plaque pathology together with axonal impairments and gliosis similar to PDAPP mice. Significant plaque pathology in Tg2576 mice can be observed at the age between 11 and 13 months. Behavioural impairments can be observed prior to the massive plaque depositions (Elder et al. 2010; Holcomb et al. 1998; Hsiao et al. 1996).

Several other APP-based models, which contain one or more FAD mutations have been developed and characterized. In summary, they show elevated A $\beta$ levels, extracellular plaque pathology, gliosis, dystrophic neurites and signs of inflammation. Furthermore, behavioural and cognitive impairment have been also frequently observed (Chishti et al. 2001; Elder et al. 2010; Games et al. 2006; Sturchler-Pierrat et al. 1997). Recently, a human APP knockin mouse model has been described. It develops amyloid pathology and memory deficits expressing the mutant APP at endogenous levels (Nilsson et al. 2014).

The mutations in presenilins 1 and 2 also have been taken to develop transgenic mouse models. Mouse models harbouring these transgenes exhibit elevated levels of $A \beta_{\mathrm{X}-42}$ but do not develop plaque pathology (Cavanaugh et al. 2014). The absence of plaques can be explained through the lower levels of $A \beta_{\mathrm{X}-42}$ in the PSEN-mutated lines comparing to APP models, or through the different solubility of human and murine A $\beta$ (Jankowsky et al. 2007).

In order to intensify the pathological phenotype, mouse models with a combination of multiple APP mutations or APP and PSEN mutations were created. The APP/PS1 $\triangle E$ 9 model (Borchelt et al. 1997), the APP/PS1KI model (Casas et al. 2004) and the 5XFAD model (see 1.7.1) are well-characterized. They display an earlier onset of pathology and more abundant plaque deposition (Elder et al. 2010). Research on these mouse models significantly contributed to the better understanding of neuron loss, behavioural phenotype and the role of intracellular A $\beta$ (Casas et al. 2004; Christensen et al. 2010; Jawhar et al. 2012; Oakley et al. 2006; Oddo et al. 2003; Wirths and Bayer 2012; Wirths et al. 2009). Intraneuronal $A \beta$ has been reported in a variety of mouse models including $\mathrm{Tg} 2576,3 \mathrm{xTg}$, APP/PS1K1 and 5XFAD (Billings et al. 2005; Casas et al. 2004; Oakley et al. 2006; Oddo et al. 2003; Takahashi et al. 2013). Several mouse models also showed high correlation between intraneuronal $A \beta$ accumulation and neuron loss (Wirths and Bayer, 2012). 
The relevance of mutant APP/PS transgenic models is sometimes called in question, because the mutations are clearly associated only with FAD, which makes up barely $1 \%$ of entire AD cases. Regarding this fact, it is reasonable to create a model for sporadic AD. However, until now there has been nearly no success in these endeavours. Unfortunately, rodents do not develop AD pathology spontaneously during the aging process (Sarasa and Pesini 2009). Nevertheless, one non-transgenic mouse model with some important pathological characteristics of sporadic AD including memory impairment has been described. The mice were subjected to intracerebroventricular (icv) injection of streptozotocin (STZ) in order to induce insulin resistant state in the brain. Diabetes mellitus type II as a probable risk factor for AD could be observed in this icv-STZ mouse model. Indeed, the icv-STZ mice show cognitive deficits, decreased energy metabolism, altered synaptic proteins, increased hyperphosphorylated Tau and signs of neuroinflammation in the brain (Chen et al. 2012; Chen et al. 2013; de la Monte and Wands 2008; Salkovic-Petrisic et al. 2006).

Besides the icv-STZ, a novel model of sporadic AD, Tg4-42, was recently generated in our group (Bouter et al. 2013a) (see 1.7.2). This model supports the hypothesis that intracellular soluble aggregates of $\mathrm{A} \beta$ play a crucial role in $\mathrm{AD}$.

Numerous species such as rats, rabbits, dogs and primates have been proposed for research purposes in the field of $\mathrm{AD}$, but mice still remain the most commonly used vertebrates in this sphere. Mice have many advantages such as a relatively short reproductive cycle, large litters and easy handling (Sarasa and Pesini, 2009). Mouse models of AD are very important for the research and contribute a lot to the understanding of etiology and pathology of Alzheimer's disease. Unfortunately, the entire clinical and neuropathological pictures of familial or sporadic AD in humans cannot be fully reconstructed in any of the available models. Anyway, showing pathological features of AD such as amyloid deposition, neuron loss, aggregation of phosphorylated Tau and behavioural and/or memory deficits, mice models reflect various aspects of the disease and therefore help us to get more insights in its pathogenesis and enable making next steps towards developing a therapy against it.

\subsubsection{The 5XFAD mouse model}

The 5XFAD mouse model was first described by Oakley et al. (2006). This model is a double transgenic APP/PS1 mouse model expressing in total five familial AD mutations (5XFAD): human APP (isoform $\mathrm{APP}_{695}$ ) carrying the Florida (I716V), London (V717I) and Swedish (K670N/M671L) mutations, and mutant PSEN1 (M146L; L286V), all under the control of a murine Thy-1 promoter (Vidal et al. 1990; Moechars et al. 1996; Oakley et al. 2006) (Figure 1.13). These mice display stable germline transmission and coinheritance of the APP and PSEN-1 transgenes over multiple generations, so these mice can be bred as if they are a single transgenic mouse model (Eimer and Vassar 2013; Oakley et al. 2006).

In the 5XFAD mouse model, the Florida, London and the two PSEN mutations lead to increased production of $A \beta_{x-42}$ species, whereas the Swedish mutation leads to elevated levels of total $A \beta$. 

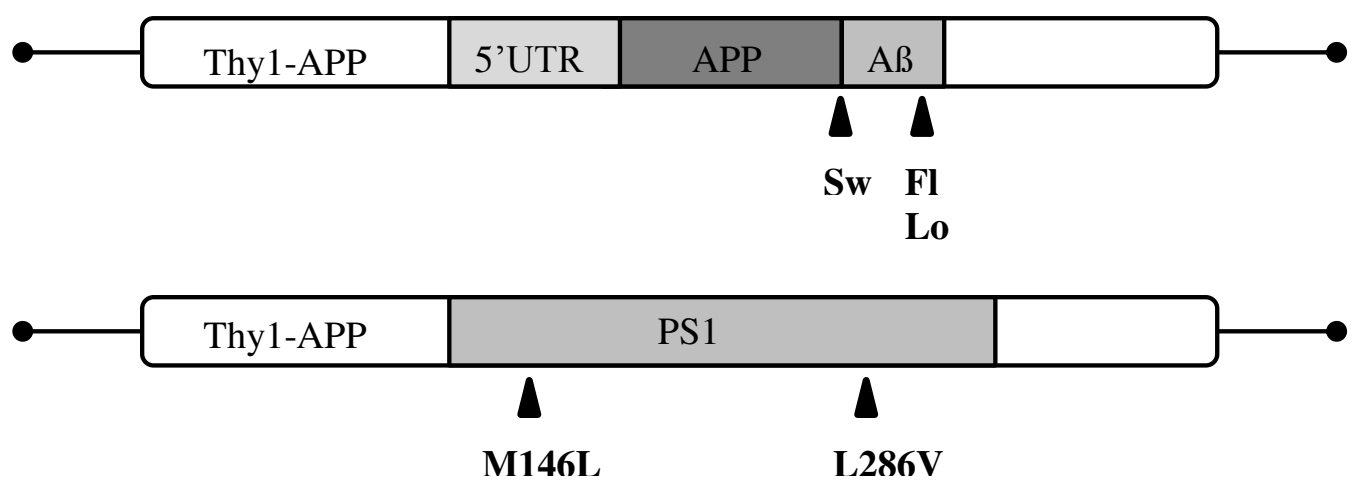

Figure 1. 13: 5XFAD transgenes. Schematic diagram of 5XFAD APP and PS1 transgenes. FAD mutations are indicated by arrowheads. Abbreviations: Sw, Swedish mutation; Lon, London mutation; Fl, FL mutation; 5'UTR, 5' untranslated region. Small grey rectangles represent Thy1 exons. Coding regions of APP and PS1 are displayed by larger rectangles inserted into Thy 1 exon 2 of the mouse Thy 1 transgene expression cassette. Figure created after Oakley et al. 2006, p. 10131.

5XFAD mice develop their pathological phenotype rapidly and display key features of AD. Plaque pathology starts at the age between two and three months and is accompanied by gliosis. These characteristics massively increase with age (Oakley et al. 2006). 5XFAD mice also display extracellular amyloid depositions in spinal cords at three months of age (Jawhar et al. 2012). Besides extracellular plaques, intracellular A $\beta$ can be observed in the cortical Layer 5 and the subiculum starting at the age of six weeks (Eimer and Vassar 2013) (Oakley et al. 2006). Intraneuronal A $\beta$ appears prior to plaque formation and correlates well with neuron loss in these brain regions (Oakley et al. 2006; Jawhar et al. 2012). Furthermore, the 5XFAD mouse shows significant neuron loss. Design based stereology revealed a significant neuron loss in the pyramidal neurons of the Layer 5 cortex and the subiculum starting at the age of 9 months (Eimer and Vassar 2013). Interestingly, neither intraneuronal $A \beta$ nor neuron loss could be found in the CA1 region of the hippocampus, despite presence of amyloid plaques in this region (Jawhar et al. 2012). The 5XFAD model also develops age-dependant synaptic degeneration and dystrophic neurites (Oakley et al. 2006). Dystrophic changes in these structures are thought to be associated with the deposition of extracellular A $\beta$ plaques (Zhang et al. 2009b).

Moreover, 5XFAD mice develop age-dependant progressive behaviour deficits. At the age of roughly 6 months, 5XFAD mice show behavioural impairment and working memory deficits. Reference memory impairments are found the age of 12 month (Oakley et al. 2006; Jawhar et al. 2012). The mice also demonstrate decreased anxiety. Impairments in the Y-maze, Morris water maze, the conditioned taste aversion task and contextual fear conditioning were observed in the 5XFAD model (Devi and Ohno 2010; Devi and Ohno 2013; Jawhar et al. 2012; Ohno et al. 2006). Besides cognitive impairments, 5XFAD mice also exhibit motor deficits, which can be linked to extensive spinal cord pathology, including dystrophic neurites and intracellular A $\beta$ accumulation (Jawhar et al. 2012).

5XFAD is a widely used model in Alzheimer's research. Several groups used this model in preclinical studies investigating possible treatments and their effects on the progression of cognitive impairment (Aytan et al. 2013; Bhattacharya et al. 2014; Cho et al. 2014; Fiol-deRoque et al. 2013; Hillmann et al. 2012; Wirths et al. 2010a). 


\subsubsection{The Tg4-42 mouse model}

The Tg4-42 model is a transgenic mouse model for sporadic Alzheimer's disease, which was generated in our group (Bouter et al. 2013a; Bouter et al. 2013b). This model is the first model, which expresses $\mathrm{N}$-truncated human $A \beta_{4-42}$ without any other mutation and still develops age-dependant neuronal loss and behavioural deficits (Bouter et al. 2013a). $\mathrm{A} \beta_{4-42}$, as mentioned in 1.6.5, is one of a variety of $\mathrm{N}$-terminally truncated $\mathrm{A} \beta$ species identified in the AD brain and may be a particularly abundant $\mathrm{A} \beta$ isoform in the human brain (Masters et al. 1985; Lewis et al. 2006; Portelius et al. 2010).

The genetic construct of $\mathrm{Tg} 4-42$ contains the $\mathrm{A} \beta_{4-42}$ sequence fused to the murine thyrotropin-releasing hormone (TRH) signal peptide under the control of the neuronal Thy-1 promoter (Figure 1.14). This allows the $A \beta$ to be secreted into the extracellular space in a neuron-specific manner (Bouter 2014).

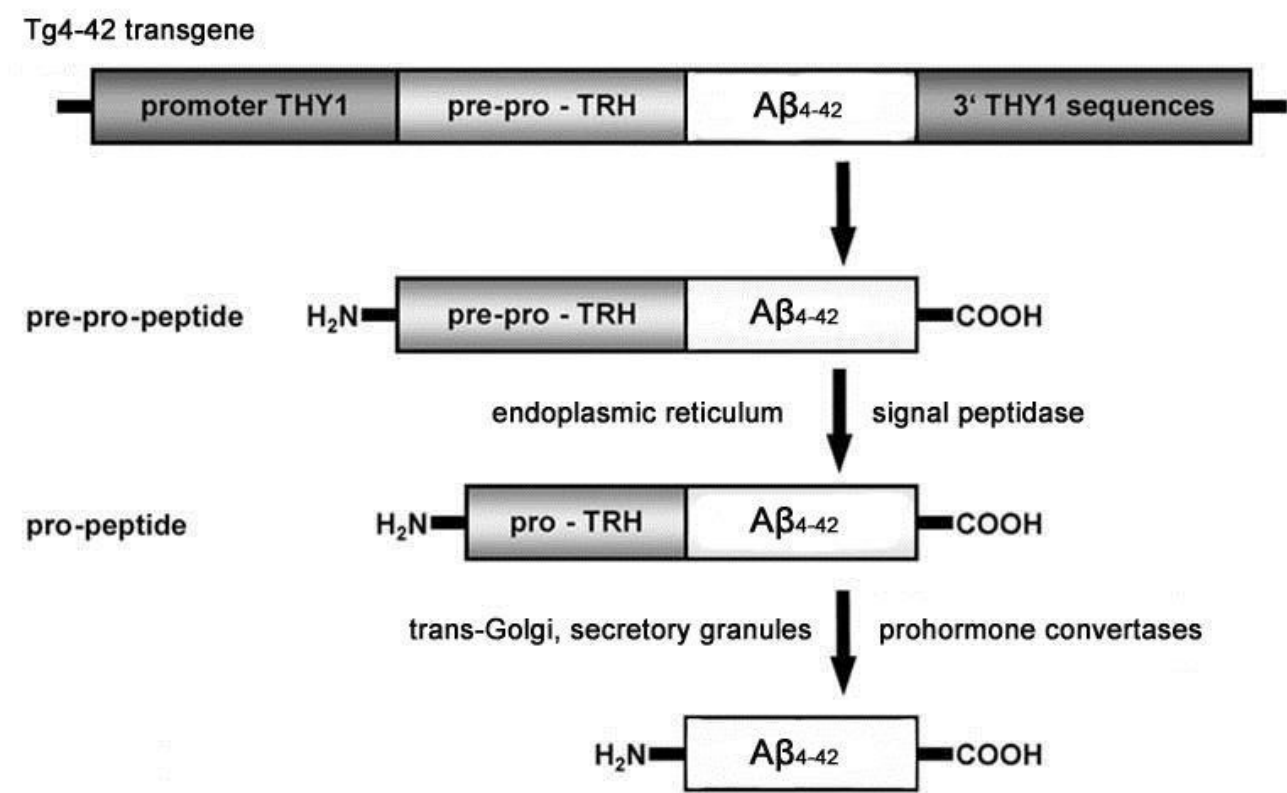

Figure 1. 14: Genetic construct of the Tg4-42 transgene mouse. The murine Thy 1 promoter drives the neuronal expression of the pre-pro-TRH-A $\beta_{4-42}$ fusion peptide. In the construct of $\mathrm{Tg} 4-42, \mathrm{~A} \beta 4-42$ is fused to pre-pro-TRH for product liberation within the secretory pathway. An $\mathrm{N}$-terminal signal sequence guides the pre-pro-TRH-A $\beta_{4-42}$ fusion peptide into the endoplasmic reticulum, where signal peptidases cleave it and release the pro-TRH-A $\beta 4-42$ peptide. Prohormone convertases in the trans-Golgi and secretory vesicles cleave the rest of the TRH signal peptide to release $A \beta_{4-42}$. Figure modified from Alexandru et al. 2011, p. 12792 and Bouter 2014, p. 31.

The transgene expression is highest in the hippocampus, mainly in the CA1 pyramidal cell layer, and is detectable starting at the age of two months. $A \beta_{4-42}$ could be also found in occipital cortex, piriform cortex, striatum, and superior colliculus. The Tg4-42 model does not express human APP, so the effects of the $A \beta_{4-42}$ peptide are not disturbed by the influence of APP or other cleavage products.

The secreted $A \beta_{4-42}$ is a highly toxic peptide. It has strong propensity to forms soluble, neurotoxic aggregates triggering neuron death in primary cortical neurons. The $\mathrm{Tg} 4-42$ 
mice do not develop any extracellular amyloid plaques; NTFs are also absent. However, the Tg4-42 mice develop age- and dose-dependent neuronal loss in the hippocampus CA1 region of hemizygous and homozygous mice, as determined by unbiased stereology. In addition, reactive microglia and astrocytes can be found in the hippocampus starting at the age of two months. Behaviourally, the Tg4-42 mice show strong spatial reference memory deficits as observed in the Morris water maze test. Memory deficits are age-dependent and are not detectable at the age of three months. The impaired spatial reference memory in Tg4-42 is compatible with AD-typical age-dependent cognitive decline (Bouter et al. 2013a; Bouter et al. 2013b; Bouter et al. 2014).

Taken together, the Tg4-42 model is a unique mouse model for sporadic AD. All other transgenic models that develop all pathologies mentioned above have at least one mutation. The Tg4-42 model has no mutations and represents the first mouse model expressing exclusively $\mathrm{N}$-truncated $A \beta_{4-42}$. Therefore, the effects of this peptide can be investigated without interference from other human APP products. Another advantage of this model is the absence of amyloid plaques, which do not correlate well with cognitive decline in $\mathrm{AD}$ patients.

\section{$1.8 \quad$ Therapeutic approaches}

\subsubsection{Pharmacological interventions}

At the present time, there is no cure for Alzheimer's disease. All current medications aim at mitigating the symptoms. Available drugs such as memantine and different acetylcholinesterase inhibitors do not target the underlying cause, they only bring some relief.

The first class of drugs used in the treatment of AD became available in the late 90s. It was acetylcholinesterase inhibitors, which aim at the effects of degeneration of cholinergic neurons and subsequent exhaustion of the neurotransmitter acethylcholine. It is known that the cholinergic system is involved in memory and behavior processes (Bartus et al. 1982). Thus, it is held that impairments in cholinergic neurons in AD lead to memory and cognitive deficits (Terry and Buccafusco 2003).

There are different drugs that inhibit the catalytic function of acetylcholinesterase enzyme that degrades acetylcholine in the synaptic cleft, but the opinions are controversial about their efficacy (Pepeu and Giovannini 2009). Among them, donepezil (Rogers and Friedhoff 1996), rivastigmine (Polinsky 1998), and galantamine (Raskind et al. 2000) are routinely prescribed. There is some evidence that the acetylcholinesterase inhibitors can temporarily delay the progression of the disease or moderate the symptoms. The result of the treatment depends on how early the treatment is started and on the responsiveness of the patients. However, none of these three drugs can cure or prevent the progression of the clinical symptoms (Bullock et al. 2005; Bullock and Dengiz 2005; Rogers et al. 1998; Wallin et al. 2011). 
Another type of drugs used in AD treatment is memantine. Memantine is a noncompetitive $N$-methyl-D-aspartate (NMDA) receptor antagonist that is prescribed to patients with moderate to severe AD (Tariot et al. 2004). It protects the nerve cells against glutamate, which is released in excess by AD-damaged brain cells (Parsons et al. 2007). Memantine has some beneficial effects in patients with $\mathrm{AD}$ and is used in combination with cholinesterase inhibitors (McShane et al. 2006; Orgogozo et al. 2002; Parsons et al. 2013; Peskind et al. 2006). All pharmacological interventions mentioned above showed similar limited effects in humans and mice (Yuede et al. 2007), supporting the validity of animal models for AD research. Furthermore, a variety of clinical trials focused on the reduction of neuroinflammation were performed (Meinert et al. 2009), as well as immunization against toxic $A \beta$ species (Wisniewski 2009).

The intracellular amyloid cascade hypothesis suggests that modulating the production or increasing the clearance of $A \beta$ could open new possibilities in treatment of AD. It was tried to inhibit or modulate the enzymes that are involved in the amyloidogenic pathway. However, up to date substrates specific but non-toxic $\gamma$-secretase inhibitors have not been developed. Inhibitors of $\beta$-secretase are also at a very early developmental stage.

Therefore, the research in the field of active and passive immunization gains a higher importance (Lannfelt et al. 2014).

Most of the immunization approaches against $A \beta$ have substantial disadvantages such as negative side effects or a lack of efficacy in humans. The remarkable effects of active immunization against A $\beta$ in the PDAPP mouse model led to the initiation of clinical trials of active immunization (Dodart et al. 2002; Janus et al. 2000; Kotilinek et al. 2002; Morgan et al. 2000). Except some patients in the first clinical phase of study failed to develop detectable antibody titers, no adverse effects were observed (Schenk 2002). Unfortunately in the clinical phase $2 \mathrm{a}$, severe side effects of active immunization such as CNS inflammation and pulmonary embolism (Ferrer et al. 2004; Nicoll et al. 2003) occurred and the study was halted. The side effects have been attributed to an autoimmune response (Orgogozo et al. 2003).

Many researchers see the future in passive immunization strategies (Hock et al. 2002). Several antibodies have been proposed for passive immunization. For clinical trials, they have been

humanized to avoid immune-responses in human patients. Passive immunization against $\mathrm{A} \beta$ has been successfully tested in murine models during the past years. It was shown that peripherally administered antibodies are able to clear $A \beta$ from murine brain (Bard et al. 2003; DeMattos et al. 2001; Frost et al. 2012; Wilcock et al. 2003; Wilcock et al. 2004a; Wilcock et al. 2004b; Wilcock et al. 2006).

However, passive immunization also caused side effects such as microhemorhages (DeMattos et al. 2012; Lannfelt et al. 2014; Pfeifer 2002; Racke et al. 2005; Schroeter et al. 2008; Wilcock et al. 2004b; Wilcock et al. 2006). Some antibodies showed an effect in the reduction of the cognitive decline but without statistical significance. However, none of these antibodies have been proven to substantially ameliorate the deficits in AD or influent progression of symptoms (Lannfelt et al. 2014).

In the recent years, the role of soluble toxic $A \beta$ aggregations and some of its $\mathrm{N}$ truncated isoforms in the pathogenesis of $\mathrm{AD}$ have attracted a lot of attention. Now it is 
widely accepted that $A \beta_{\mathrm{PE} 3-\mathrm{X}}$ may play an important role in $\mathrm{AD}$. Another abundant $\mathrm{N}$ truncated species in human AD brain is $A \beta_{4-X}$ (Bouter et al. 2013a; Bouter et al. 2014). The first studies of anti-A $\beta_{\mathrm{pE} 3-\mathrm{X}}$ immunotherapy in mice had promising outcomes (DeMattos et al. 2012; Frost et al. 2012; Wirths et al. 2010a). Therefore, N-truncated A $\beta$ isoforms became of a great interest as possible therapeutic targets. Passive immunotherapy against $A \beta$ species is an important topic of ongoing research and remains a promising option to develop an efficient treatment of AD.

However, considering the fact that there are still no drugs that can cure AD, the importance of preventing approaches grows immensely. There are a variety of studies confirming that a healthy and physically active lifestyle together with cognitive stimulating activities and a Mediterranean diet reduce risk for Alzheimer's disease.

\subsubsection{Environmental interventions}

A variety of epidemiological studies underline the beneficial effects of different environmental factors, such as physical activity (Hillman et al. 2008; Lautenschlager et al. 2008), participation in leisure activities (Crowe et al. 2003; Akbaraly et al. 2009), intellectual and cultural activities (Cummings et al. 1998) and a healthy balanced diet (Feart et al. 2015) (Willett 2006) show indisputable benefits in older adults at risk for AD (Knopman 2009).

Feart et al. (2015) reviewed recent epidemiolocal studies concerning the Mediterranean diet. The Mediterranean diet consists of vegetables, fruits, low fat meats such as fish and poultry, polyunsaturated fats such as olive oil, and unrefined cereals. The Mediterranean diet has protective effect on cognitive health, including a reduced risk of Alzheimer's disease and cognitive impairment. Overall, it was suggested that the Mediterranean diet might exert a long-term beneficial effect on brain functioning (Willett 2006).

One of the widely accepted explanations of beneficial effects of environmental interventions is the cognitive reserve (CR) hypothesis. According to Steffener and Stern (2012), cognitive reserve is an ability to make flexible and efficient use of available brain reserve when performing tasks. CR has been most often estimated using IQ and education. Other variables have also been used (e.g. literacy, occupational complexity, participation in leisure activities, cohesion of social networks, personality variables, socioeconomic status, and aerobic fitness). Individuals with higher cognitive reserve tend to have better clinical outcomes for any level of pathology and brain reserve ${ }^{3}$ (Steffener and Stern 2012; Stern 2002; Stern 2006; Stern 2009; Hillman et al. 2008). It was suggested that environmental interventions promote a better CR that helps coping with pathological changes in brain during the Alzheimer's disease (Akbaraly et al. 2009; Albert et al. 1995).

\footnotetext{
${ }^{3}$ Steffener and Stern (2012) have suggested that two types of reserve contribute to maintaining functioning in the presence of brain changes or insult: brain reserve and cognitive reserve. Brain reserve is the reserve, which derives from brain size or neuronal cell number (e.g. larger brains can sustain more insult before clinical deficit emerges, because sufficient neural substrate remains to support normal function).
} 
A growing body of evidence suggests that also lifelong bilingualism may delay the onset of AD by several years (Gold 2015). It was shown in epidemiological studies that individuals who speak two languages on a regular basis since childhood tend to develop clinical AD symptoms at an older age than monolinguals. Thus, bilingualism is very interesting as a potential form of $\mathrm{CR}$, primary because it appears to be a primarily environmental factor for which neither special education nor intelligence is needed (Bialystok et al. 2007; Alladi et al. 2013; Gold 2015; Lawton et al. 2015).

Physical exercises promote reconfiguration of brain networks, which is essential in learning, memory, and executive functions (Foster 2015). In wild-type rodents, it was shown that physical activity increases cell proliferation and adult hippocampal neurogenesis (Blackmore et al. 2009; Couillard-Despres et al. 2005; van Praag et al. 1999), and improves learning (Stranahan and Mattson 2008). In spite of evidence for the importance of physical activity, $74 \%$ of adults in the United States have sedentary lifestyle and do not meet the recommended guideline of at least 30 minutes of moderate-intensity physical daily activity. Recent epidemiological studies indicate that children are also growing increasingly unfit, and that these lifestyle factors are related to an earlier onset of a variety of chronic diseases. Numerous studies also showed that participation in physical activity has been associated also with the decrease of a number of physical (e.g. cardiovascular disease, cancer diseases, obesity) and mental (e.g. dementia, depression and anxiety) disorders during the life. (Hillman et al. 2008).

The environmental enrichment (EE) is an experimental setting in which the animals are housed in cognitively and physically stimulating conditions. It is used in mouse models to simulate stimulating human living conditions (e.g. social interactions, sensory and motor stimulation).

The pathological phenotype of several transgenic mouse models of AD exposed to environmental enrichment was significantly improved. Decreased A $\beta$ levels and inflammation markers were found in the environmentally enriched Tg2576 mice (Nichol et al. 2007, S. 2; Nichol et al. 2008; Stranahan et al. 2012). Spatial memory was improved and Alzheimer's disease-like pathology was alleviated in senescence-accelerated prone-8 (SAMP8) mice (Dong et al. 2012). Enriched environment also ameliorated performance in cognitive tasks in the PS1/PDAPP (Costa et al. 2007). In the TgCRND8 mice, a decrease of amyloid deposition has been observed after EE (Adlard et al. 2005). It was also shown in TgCRND8 mice that cognitive and physical stimulation increases neurogenesis in hippocampus to wild type levels. Furthermore, EE prevents astrogliosis in hippocampus of adult PDAPP-J20 and 3xTg transgenic mice (Beauquis et al. 2013; Rodríguez et al. 2013). EE induced also beneficial effects on working memory in 3xTg mice (Blázquez et al. 2014). In addition, EE rescues adult neurogenesis in the APP23 mouse model of AD (Mirochnic et al. 2009). However, EE failed to ameliorate SGZ neurogenesis in APP/PS1K1 as well as neuropathological alterations and memory deficits (Cotel et al. 2012). Positive effects due to EE have also been reported in sporadic models of Alzheimer such as APOE $\varepsilon 4$ mice (Nichol et al. 2009). It was suggested that EE improves diminished cellular plasticity in AD brain via enhancing the brain capacity to better compensate for neurodegeneration (Herring et al. 2009). Due to its neurogenic effect, EE was considered to be a potential therapeutic approach in AD (Schaeffer et al. 2009; Um et al. 2008). 


\subsection{Aims of the study}

The experimental work presented in the following pages aimed to further investigate two mouse models of Alzheimer's disease: Tg4-42 and 5XFAD. Another goal of my doctoral study was to determine the effects of environmental intervention on histopathological features of the $\mathrm{Tg} 4-42$ mice.

The present study consists of two projects. The aim of the first project was to further characterize the new model for sporadic $\mathrm{AD}, \boldsymbol{T g}$ 4-42 expressing $\mathrm{A} \beta_{4-42}$, which was recently developed in our group. Because of the variety of typical for AD symptoms displayed by this unique model, it is of great interest to evaluate the effects of physical, social and sensorial stimulation.

Therefore, groups of $\mathrm{Tg} 4-42$ transgenic females were exposed to $\mathrm{EE}$ at an early age and in a continuous manner. They were subsequently tested for hippocampus-dependent memory, motor abilities, anxiety levels (projects of my colleagues), and their cerebral tissue was thoroughly analysed using unbiased stereology for neurogenesis and neuronal loss in the dentate gyrus (present study). Groups of Tg4-42 mice housed under enriched environmental conditions were compared to the age-matched $\mathrm{Tg} 4-42$ and wild-type mice from the standard housing. The dentate gyrus region was chosen, because it is located in the centre of the hippocampal formation, which is severely affected in AD. The hippocampus is thought to be involved in memory and learning processes. In addition, the dentate gyrus is a unique region, in which adult neurogenesis takes place. Adult hippocampal neurogenesis is a process involving the continuous generation of newborn neurons in in the subgranular zone of the dentate gyrus. Mounting evidence has suggested that hippocampal neurogenesis contributes to some forms of hippocampus-dependent learning and memory and therefore may be a potential therapeutic target in AD treatment.

The second project aimed at investigation the gene dosage dependence of the neuropathological features in the widely used mouse model for familial Alzheimer's disease 5XFAD.

Therefore a homozygous 5XFAD strain was created to elevate transgene expression and $\mathrm{A} \beta$ production. In order to evaluate the gene dosage-dependent effect on the extent of amyloidosis and axonal impairments, hemizygous and homozygous mice were compared. Sagittal brain sections of hemizygous and homozygous 5XFAD mice at 2, 5 and 9 months of age were stained with an $A \beta[N]$ antibody, which detects an $\mathrm{N}$-terminal $\mathrm{A} \beta$ epitope, and the amyloid burden was quantified in the cortex, hippocampus (hippocampal region (CA and DG), retrohippocampal region (subiculum)) and thalamus. In addition, axonal swellings located in the pons and spinal cord were counted in order to evaluate the extent of axonal pathology. 


\section{MATERIALS AND METHODS}

\subsection{Animals}

\subsubsection{General considerations}

All animals used in this study were of the species Mus musculus. Wild-type mice were of the inbred strain C57Bl/6J (Jackson Laboratories, Bar Harbor, ME, USA). The mice were housed under specific-pathogen-free conditions at the central animal facility of the Universitätsmedizin Göttingen (the Göttingen University Medical Centre) in standardized laboratory cages (described in 2.1.4). Food pellets and fresh tap water have been provided ad libitum. All animals were handled according to the guidelines of the "Society for Laboratory Animals Science" (GV-SOLAS) and the guidelines of the "Federation of European Laboratory Animal Science Association" (FELASA). The experiments were approved by the "Landesamt für Verbraucherschutz und Lebensmittelsicherheit" (LAVES) Niedersachsen (Lower Saxony State Office for Consumer Protection and Food Safety). All efforts were made to reduce the number of animals used for this study and to minimize their suffering. Individuals that suffered from massive loss of weight $(>20 \%)$ or other severe conditions such as blind eyes were sacrificed immediately and excluded from analyses.

Unless otherwise specified, males and females were used for analyses. The mouse models used in this study are listed in table 2.1.

Table 2. 1: Mouse models used in the current study

\begin{tabular}{|l|l|l|l|l|}
\hline Mouse model & Gene & Protein & Mutations & Promotor \\
\hline Tg 4-42 & APP & $\begin{array}{l}\text { N-truncated } \\
\text { A } \beta 4-42\end{array}$ & absent & murine Thy-1 \\
\hline $5 x F A D$ & APP & APP695 & $\begin{array}{l}\text { Swedish (K670N, M671L), } \\
\text { Florida (I716V), London } \\
\text { (V717I) }\end{array}$ & murine Thy-1 \\
& PSEN1 & PS1 & M146L, L286V & murine Thy-1 \\
\cline { 2 - 6 } & & & & \\
\hline
\end{tabular}




\subsubsection{Tg4-42 transgenic mice}

The Tg4-42 model is a transgenic mouse model, which reflects the sporadic form of Alzheimer's disease. This model was previously generated in our lab. Tg4-42 mice do not have any mutations but show neurological deficits due to transgenic overexpression of human N-truncated-A $\beta_{4-42}$ fused to the murine TRH signal peptide under the control of the neuronal Thy-1 promoter (Bouter et al. 2014; Wittnam et al. 2012) (see 1.7.2). The homozygous Tg4-42 mouse line ( $\mathrm{Tg} 4-42_{\text {hom }}$ ) was also generated in our lab and has been used in the current study (see table 2.2). In order to produce potential homozygous mice, hemizygous Tg4-42 mice were crossed. In order to validate homozygosity, quantitative Real-Time PCR was performed on DNA extracted from mouse tails followed by backcrossing of presumptive homozygous mice with wild-type mice to verify the true homozygosity. After homozygosity of the males and females was confirmed, they were re-mated to generate Tg4-42 $2_{\text {hom }}$ mice.

Table 2. 2: Tg4-42 and wild-type mice used in the current study

\begin{tabular}{|l|l|l|l|l|l|}
\hline \multicolumn{1}{|c|}{ Genotype } & $\begin{array}{c}\text { Living } \\
\text { conditions }\end{array}$ & \multicolumn{1}{c|}{$\begin{array}{c}\text { Age } \\
\text { (months) }\end{array}$} & \multicolumn{1}{|c|}{ Sex } & $\begin{array}{c}\text { Number of } \\
\text { animals used }\end{array}$ & $\begin{array}{c}\text { Left hemi- } \\
\text { sphere }\end{array}$ \\
\hline $\mathrm{Tg} 4-42_{\mathrm{hom}}$ & $\mathrm{EE}$ & 6 & female & 11 & + \\
\hline $\mathrm{Tg} 4-42_{\mathrm{hom}}$ & SH & 6 & female & 4 & + \\
\hline $\mathrm{Tg} 4-42_{\mathrm{hom}}$ & SH & 6 & male & 4 & + \\
\hline Wild-type $(\mathrm{C} 57 \mathrm{~B} 1 / 6 \mathrm{~J})$ & SH & 6 & female & 8 & + \\
\hline
\end{tabular}

\subsubsection{XFAD transgenic mice}

The 5XFAD model was initially generated and described by (Oakley et al. 2006) (see 1.7.1). 5XFAD mice used in this study were maintained on a C57B16/J genetic background (Jackson Laboratories, Bar-Harbor, ME, USA) (Jawhar et al. 2012). 5XFAD transgenic mice express five familial AD mutations (5XFAD): human amyloid precursor protein (APP695) carrying the Swedish (K670N, M671L), London (V717I) and Florida (I716V) mutations and human presenilin-1 (PSEN1) carrying the M146L/L286V mutations, all under the control of the murine Thy1-promoter (Moechars et al. 1996; Oakley et al. 2006; Vidal et al. 1990). These five mutations are known to cause familial AD in humans.

To generate a homozygous 5XFAD line, heterozygous 5XFAD mice were crossed. The offspring was analysed using quantitative Real-Time PCR of genomic DNA obtained from the mouse tails for human APP with murine APP as a calibrator. 5XFAD mice, which showed an approximately doubled human APP gene dosage compared to $5 \mathrm{XFAD}_{\text {hem }}$ mice, were identified as potential $5 \mathrm{XFAD}_{\text {hom }}$ mice. Homozygosity of potential $5 \mathrm{XFAD}_{\text {hom }}$ mice was confirmed by backcrossing with wild-type mice before they were used for further breeding (Richard et al. 2015). 
Only female hemizygous and homozygous 5XFAD mice were used for the neuropathological analyses at 2 and 5 months of age. For the analysis at 9 months of age, only male mice were used (table 2.3).

Table 2. 3: 5XFAD mice used in the current study

\begin{tabular}{|c|l|l|l|l|l|}
\hline Genotype & $\begin{array}{c}\text { Age } \\
\text { (months) }\end{array}$ & \multicolumn{1}{|c|}{ Sex } & $\begin{array}{c}\text { Number of ani- } \\
\text { mals used }\end{array}$ & $\begin{array}{c}\text { Right hemi- } \\
\text { sphere }\end{array}$ & Spinal cord \\
\hline \multirow{3}{*}{$5 \mathrm{XFAD}_{\text {hom }}$} & 2 & female & 4 & + & - \\
\cline { 2 - 7 } & 5 & female & 7 & + & - \\
\cline { 2 - 7 } & 9 & male & 4 & + & + \\
\hline \multirow{5}{*}{$5 \mathrm{XFAD}_{\text {hem }}$} & 2 & female & 6 & + & - \\
\cline { 2 - 7 } & 5 & female & 8 & + & - \\
\cline { 2 - 7 } & 9 & male & 3 & + & - \\
\cline { 2 - 7 } & 9 & male & 3 & + & - \\
\hline
\end{tabular}

\subsubsection{Housing conditions}

\subsubsection{Standard housing}

From weaning until the age of 8 weeks, all mice were housed in standardized laboratory cages (individually ventilated cages (IVC), $32 \times 16 \times 14 \mathrm{~cm}$ ) in groups of up to five individuals. The mice assigned to the standard housing ( $\mathrm{SH}$ ) living condition were kept in these cages in groups of four to five females till the date of sacrificing. Males were kept alone in separate cages in order to avoid hierarchy-related conflicts and possibly resulting injuries. This way of keeping promotes social interactions without hierarchy-related aggressive behaviour (Fitchett et al. 2005). Sawdust bedding and some nesting material were provided.

\subsubsection{Environmental enrichment}

The 8 week-old Tg4-42 mice that were assigned to the environmental enrichment (EE) living condition were transferred to rat cages $(55 \mathrm{~cm} \mathrm{x} 34 \mathrm{~cm} \mathrm{x} 20 \mathrm{~cm})$ in groups of four to five females. In contrast to SH-cages, in the larger EE-cages mice had access to a variety of objects such as running wheels, plastic toys, tunnels and igloos, which were changed and spatially rearranged every week in order to stimulate spatial cognition (O'Keefe and Nadel 1978). Running wheels were accessible all the time to promote physical activity (van Praag et al. 1999). 


\subsection{Isolation of genomic DNA and genotyping of animals}

\subsubsection{DNA isolation from mouse tails}

All mice used for the neuropathological analyses in this study were genotyped. For genotyping, genomic DNA was isolated from mouse tail biopsies. Per sample, $500 \mu \mathrm{l}$ of lysis buffer [100 mM Tris/ $\mathrm{HCl}(\mathrm{pH} 8.5), 5 \mathrm{mM}$ EDTA, 0.2\% (w/v), sodium dodecyl sulfate (SDS), and $200 \mathrm{mM} \mathrm{NaCl}$ ] and $5 \mu 1$ Proteinase K $(20 \mathrm{mg} / \mathrm{ml}$ stock) were added. Samples were incubated for 20 hours at $55^{\circ} \mathrm{C}$ in a Thermomixer Compact under gentle agitation (350-450 rpm). Afterwards, samples were centrifuged for 20 minutes at $4^{\circ} \mathrm{C}$ and 17,000 rpm. The supernatant was transferred into a new $1.5 \mathrm{ml}$ Eppendorf tube containing $500 \mu \mathrm{l}$ cold isopropanol and the pellet was discarded. Samples were vortexed then until the DNA precipitate was visible. This was followed by another 10 minutes of centrifugation at $13,000 \mathrm{rpm}$ at room temperature (RT). After centrifugation, the supernatant was discarded and the DNA containing pellet was washed in $500 \mu 170 \%(\mathrm{v} / \mathrm{v})$ ice-cold ethanol. The solutions were centrifuged one more time at 13,000 rpm for 10 minutes at RT. After the supernatant was discarded, the pellet was left to dry at $37^{\circ} \mathrm{C}$ in a Thermomixer Compact for 45 minutes. The pellet was then dissolved in $30 \mu \mathrm{l}$ molecular grade water and left overnight at $56^{\circ} \mathrm{C}$ in a Thermomixer Compact before being stored at $4^{\circ} \mathrm{C}$.

\subsubsection{Determination of DNA concentration and purity}

The DNA concentration of each sample was determined via photometry. As a blank for the photometry settings, $80 \mu \mathrm{l}$ of molecular grade water was used. An amount of $2 \mu \mathrm{l}$ of each sample was diluted with $78 \mu \mathrm{l}$ molecular grade water in a Uvette ${ }^{\circledR} 220-1600 \mathrm{~nm}$. The A260/A230 absorbance ratio and the A260/A280 absorbance ratio were measured in the photometer for each DNA sample. DNA samples with A260/A230 and A260/A280 > 1.8 were considered sufficiently pure (Barbas et al. 2007). Afterwards, samples were diluted to a concentration of $20 \mathrm{ng} / \mu \mathrm{l}$ in molecular grade water.

\subsubsection{Genotyping of animals using polymerase chain reaction (PCR)}

The extracted and diluted genomic DNA from mouse tails, was used for genotyping. 5XFAD and Tg4-42 mice carrying the transgene were identified using conventional polymerase-chain-reaction (PCR).

PCR reactions were performed in a LabCycler using $0.2 \mathrm{ml} \mathrm{PCR}$ tubes. The DNA (2 $\mu \mathrm{l})$ was given into a PCR reaction tube and the prepared master mix $(18 \mu \mathrm{l})$ was added. A master mix consisted of 10x reaction buffer, $25 \mathrm{mM}$ magnesium chloride, Taq polymerase ( $5 \mathrm{U} / \mu \mathrm{l})$, dNTPs, molecular grade water and the respective primers. All primers were used at a concentration of $10 \mathrm{pmol} / \mu \mathrm{l}$ (1:10 dilution of the $100 \mathrm{pmol} / \mu \mathrm{l}$ primer stock prepared in 
$\mathrm{ddH}_{2} \mathrm{O}$ ). All primers were purchased from Eurofins (Ebersberg, Germany) as intronspanning validated primer pairs. The primers used in this study are given in the table 2.4.

Reaction mixes for the PCR and cycling conditions are listed in the table 2.5, table 2.6 and table 2.7 .

Table 2. 4: List of primers used for mouse genotyping.

\begin{tabular}{|l|l|}
\hline Primer & Sequence $\left(\mathbf{5}^{\prime} \rightarrow \mathbf{3}^{\prime}\right)$ \\
\hline A $\beta 3-42$ for & GTGACTCCTGACCTTCCAG \\
\hline A $\beta 3-42$ rev & GTTACGCTATGACAACACC \\
\hline hAPP for & GTAGCAGAGGAAGAAGTG \\
\hline hAPP rev & CATGACCTGGGACATTCTC \\
\hline
\end{tabular}

Table 2. 5: Reaction mix for Tg4-42 PCR.

\begin{tabular}{|l|l|}
\hline Reagent & Volume $[\boldsymbol{\mu l}]$ \\
\hline DNA $(20 \mathrm{ng} / \mu \mathrm{l})$ & 2.0 \\
\hline $\mathrm{A} \beta 3-42$ for2 primer & 1.0 \\
\hline $\mathrm{A} \beta 3-42$ rev2 primer & 1.0 \\
\hline $\mathrm{dNTPs}(2 \mathrm{mM})$ & 2 \\
\hline $\mathrm{MgCl} 2(25 \mathrm{mM})$ & 1.6 \\
\hline $10 \mathrm{x}$ reaction buffer & 2 \\
\hline Molecular grade water & 10.2 \\
\hline Taq polymerase $(5 \mathrm{U} / \mu \mathrm{l})$ & 0.2 \\
\hline Total volume per sample & 20 \\
\hline
\end{tabular}

Table 2. 6: Reaction mix for 5XFAD PCR.

\begin{tabular}{|l|l|}
\hline Reagent & Volume $[\boldsymbol{\mu l}]$ \\
\hline DNA $(20 \mathrm{ng} / \mu \mathrm{l})$ & 2.0 \\
\hline hAPP for primer & 0.5 \\
\hline hAPP rev primer & 0.5 \\
\hline dNTPs $(2 \mathrm{mM})$ & 2 \\
\hline MgCl2 $(25 \mathrm{mM})$ & 3.2 \\
\hline $10 \mathrm{x}$ reaction buffer & 2 \\
\hline Molecular grade water & 9.6 \\
\hline Taq polymerase $(5 \mathrm{U} / \mu \mathrm{l})$ & 0.2 \\
\hline Total volume per sample & 20 \\
\hline
\end{tabular}


Table 2. 7: PCR cycling program for genotyping Tg4-42 and 5XFAD mice.

\begin{tabular}{|l|l|l|}
\hline Step & Temperature $\left.^{\circ}{ }^{\circ} \boldsymbol{C}\right]$ & Duration [s] \\
\hline 1 & 94 & 180 \\
\hline 2 & 94 & 45 \\
\hline 3 & 58 & 60 \\
\hline 4 & 72 & 60 \\
\hline 5 & \multicolumn{2}{|l|}{ Repetition of steps 2-4 (35 times) } \\
\hline 6 & 72 & 300 \\
\hline 7 & 4 & $\propto$ \\
\hline
\end{tabular}

\subsubsection{DNA agarose gel electrophoresis}

The PCR products were loaded on agarose gels to identify transgene animals using gel electrophoresis.

1x TBE buffer ( $89 \mathrm{mM}$ Tris, $89 \mathrm{mM}$ boric acid, $2 \mathrm{mM}$ ethylenediaminetetraacetic acid) was necessary for the gel electrophoresis. At first, 10x TBE buffer was made as fol-

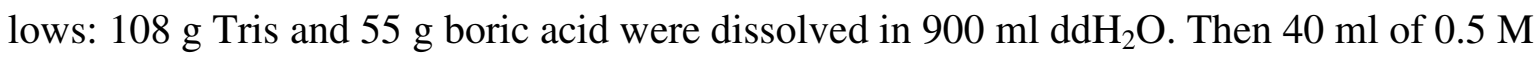
ethylenediaminetetraacetic acid ( $\mathrm{pH}$ 8.0) was added to the solution. The volume was adjusted to 11 with $\mathrm{ddH}_{2} \mathrm{O}$. The solution was diluted 1:10 in $\mathrm{ddH}_{2} \mathrm{O}$ to obtain $1 \mathrm{x}$ TBE buffer.

To prepare the gel, $100 \mathrm{ml}$ of $1 \mathrm{x}$ TBE buffer was added to $2 \mathrm{~g}$ agarose and boiled in a microwave at $560 \mathrm{~W}$ until the agarose was completely dissolved.

Afterwards, $3 \mu$ ethidium bromide $(10 \mathrm{mg} / \mathrm{ml})$ was added to the liquid gel and the gel was casted in a casting tray with a comb to form wells. Any air bubbles were removed. After the gel was cooled down, the comb was carefully removed. The gel was then positioned in an electrophoresis chamber filled with $1 \mathrm{x}$ TBE buffer. The samples were treated as follows: $10 \mu \mathrm{l}$ of the PCR product was mixed with $1 \mu 110 \mathrm{x}$ agarose gel sample buffer and loaded into a well. The gel was run in a horizontal electrophoresis chamber connected to a power. For size indication, one well was filled with $5 \mu \mathrm{l}$ of the $100 \mathrm{bp}$ DNA ladder and run in TBE buffer for approximately 45 minutes at $120 \mathrm{~V}$ constant current. The DNA in the gel was visualized under a UV light $(366 \mathrm{~nm}$ ) using the Gel Doc 2000 (Biorad, Hercules, CA, USA) and analysed with the software program Quantity One (Version 4.30; Bio$\mathrm{rad})$.

\subsubsection{Quantitative Real-Time PCR Genotyping of 5XFAD mice}

The homozygous 5XFAD mice were identified through quantitative Real-Time PCR (qRT-PCR) using a MX3000P Real-Time Cycler with $10 \mathrm{ng}$ of genomic DNA per reaction. For the quantification of the PCR product, the SYBR-green based DyNAmo Flash SYBR Green qPCR Kit (Thermo Fisher Scientific, Waltham, MA, USA) containing ROX as an internal reference dye was used. The DNA dilutions were mixed with the qPCR reaction 
mix in $200 \mu \mathrm{l}$ PCR tubes and briefly centrifuged. The reaction mix and cycling protocol are given in tables 2.8 and 2.9. The primers used for qRT-PCR are given in the table 2.10.

Table 2. 8: List of primers (Eurofins, Ebersberg, Germany) used for qRT-PCR

\begin{tabular}{|l|l|}
\hline Primer & Sequence $\left(\mathbf{5}^{\prime} \rightarrow \mathbf{3}^{\prime}\right)$ \\
\hline hAPP for & GTAGCAGAGGAAGAAGTG \\
\hline hAPP rev & CATGACCTGGGACATTCTC \\
\hline mAPP-for & TCTTGTCTTTCTCGCCACTGGC \\
\hline mAPP-rev & GCAGTCAGAAGTTCCTAGG \\
\hline
\end{tabular}

Table 2. 9: Reaction mix for 5XFAD qRT-PCR

\begin{tabular}{|l|l|}
\hline Reagent & Volume $[\boldsymbol{\mu l}]$ \\
\hline DNA $(20 \mathrm{ng} / \mu \mathrm{l})$ & 2.0 \\
\hline hAPP for or mAPP-for primer & 0.5 \\
\hline hAPP rev or mAPP-rev primer & 0.5 \\
\hline Master Mix & 10 \\
\hline ROX & 0.2 \\
\hline Molecular grade water & 6.3 \\
\hline
\end{tabular}

Table 2. 10: qRT-PCR cycling program for detecting $5 X{ }^{2} A D_{\text {hom }}$ mice

\begin{tabular}{|l|l|l|}
\hline Step & Temperature $\left[{ }^{\circ} \boldsymbol{C}\right]$ & Duration [s] \\
\hline 1 & 95 & 600 \\
\hline 2 & 95 & 15 \\
\hline 3 & 64 & 20 \\
\hline 4 & 72 & 30 \\
\hline 5 & \multicolumn{2}{|l|}{ Repetition of steps 2-4 (40 times) } \\
\hline 6 & 95 & 60 \\
\hline 7 & 55 & 30 \\
\hline & 95 & 30 \\
\hline
\end{tabular}

The reaction was performed in duplicates in separate tubes for each pair of primers. All data were collected using the MxPro Mx3000P software (Stratagene, Santa Clara, CA, USA).

Average $C_{T}$ values were determined from the duplicates. $C_{T}$ value (threshold cycle value) is the cycle value at which a statistically significant increase in fluorescence is first detected. Relative quantification was performed using murine APP as a reference gene for normalization. The transgene levels of human APP (hAPP) were normalized to those of murine APP (mAPP) and calibrated to a selected 5XFAD animal with confirmed genotype using the $2^{-\triangle \Delta C T}$ method (Livak and Schmittgen 2001):

(1) Relative Amount Gene $=2^{-\Delta \Delta C_{T}}$ 
For an animal (q), the level of hAPP gene expression was normalized to the expression of $\mathrm{mAPP}$ as a reference gene and calibrated to an animal with confirmed genotype (cb). $\Delta \Delta \mathrm{C}_{\mathrm{T}}$ is calculated as follows (2) and (3):

$$
\begin{aligned}
\Delta C_{T} & =C_{T, h A P P}-C_{T, m A P P} \\
-\Delta \Delta C_{T} & =-\left(\Delta C_{T, q}-\Delta C_{T, c b}\right)
\end{aligned}
$$

Figure 2.1 gives an example, how animals have being identified as potentially homozygous or heterozygous 5XFAD animals.

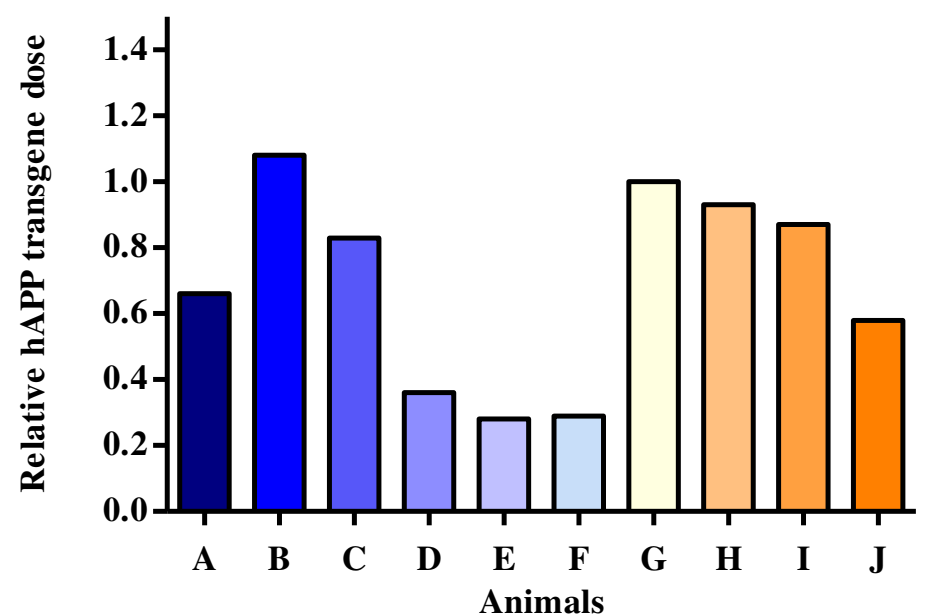

Figure 2. 1: An example for Real-Time-PCR genotyping of 5XFAD mice. Human APP gene doses in the animals A, B, C, G, H, I and J were at least twice as high in comparison to the animals D, E and F. Mice G, $\mathrm{H}$, I and $\mathrm{J}$ were previously confirmed to be homozygous. Mice A, B, C with unknown genotype were identified as homozygous and mice D, E and F were considered hemizygous. All mice were calibrated here to one random chosen homozygous mouse (here mouse G). Source: own research.

\subsection{Tissue collection and preservation}

Tissue was collected and preserved in different ways depending on the following analysis.

For neuronal stereology (cresyl violet staining) and immunostaining on frozen freefloating sections the mice were deeply anesthetized by a mixture of ketamine and xylazine diluted in molecular-grade water. The mice were anesthetized by an intraperitoneal injection at a dosage of $100 \mathrm{mg} / \mathrm{kg}$ ketamine and $10 \mathrm{mg} / \mathrm{kg}$ xylazine body weight.

Thereafter a fixation was done by transcardial perfusion through the left ventricle with $30 \mathrm{ml}$ ice-cold 0.01 M Dulbecco's phosphate buffered salt solution (PBS) in order to clear the body from blood. Afterwards perfusion was continued by $30 \mathrm{ml}$ of $4 \%$ paraformalde- 
hyde (PFA) dissolved in 0.01 M PBS. Discolouring of the liver indicated a successful perfusion.

The brains and spinal cords were carefully removed and brains were divided at the midline. The left hemispheres were post-fixed for at least 2 hours in $4 \%$ phosphatebuffered formalin at $4{ }^{\circ} \mathrm{C}$ and cryoprotected overnight in a $30 \%$ sucrose solution. When the brain was sunken to the bottom it was quickly frozen directly on a block of dry ice and stored deep-frozen at $-80^{\circ} \mathrm{C}$. The right hemispheres were embedded in paraffin as described below.

In order to collect sections for neuronal stereology and for the quantification of doublecortin-positive neurons in free-floating frozen sections, the fixed frozen brains were taken out of the $80^{\circ} \mathrm{C}$-freezer and left at $-20^{\circ} \mathrm{C}$ for at least 1 hour followed by embedding in a soluble glycol and resin compound. Immediately after this each frozen hemisphere was cut in 10 series of $30 \mu \mathrm{m}$-thick coronal sections on a cryostat. Every tenth section throughout the brain was collected in a small glass container. The glass containers with the sections were stored frozen at $-80^{\circ} \mathrm{C}$ until further processing.

For the immunohistochemical staining of the 5XFAD mice, they were deeply anesthetized as described above, then also transcardially perfused with $30 \mathrm{ml}$ ice-cold $0.01 \mathrm{M}$ PBS in order to remove the blood cells and hereby decrease background staining in immunohistochemistry. Some animals were sacrificed without perfusion via $\mathrm{CO}_{2}$ anesthetization followed by cervical dislocation. After the mouse cadavers were decapitated, brains and spinal cords were carefully dissected on ice and brains were divided at the midline. The tissue (right hemispheres and spinal cords) was placed into embedding cassettes and preserved by fixation in $4 \%$ paraformaldehyde in $0.01 \mathrm{M} \mathrm{PBS}$ at $4{ }^{\circ} \mathrm{C}$ for at least 3 days, then dehydrated and immersed in paraffin using a TP1020 Automatic Tissue Processor (Leica). Then the tissues were embedded in paraffin blocks using an EG1140 H Embedding Station (Leica). For immunohistochemistry, $4 \mu \mathrm{m}$ sagittal sections of the right hemispheres and transversal sections of spinal cords were produced by cutting paraffin blocks with a microtome and transferring tissue sections onto glass slides in a $50-54^{\circ} \mathrm{C}$ hot water bath. The slides were dried at $37^{\circ} \mathrm{C}$ for at least 10 hours before they were used for immunohistochemistry.

\subsection{Histological Stainings}

\subsubsection{Cresyl violet staining}

The frozen section series comprising every tenth section throughout the brain (one section series from each animal) were rehydrated in cold 0.01 M PBS solution in order to remove organic glue. Then, they were carefully mounted on glass slides. When dried, the sections were stained with a cresyl violet acetate solution (Powers and Clark 1955).

At first the sections were washed twice for 10 minutes in a $0.04 \mathrm{M}$ natriumacetate trihydrate $/ 0,1 \%$ acetic acid solution (1B). Thereafter the sections were delipidated for 20 
minutes in a $0,025 \%$ Triton $\mathrm{X}-100 / 75 \%$ ethanol solution (3B), washed two times for 10 minutes in $1 \mathrm{~B}$ and stained two times for 8 minutes in $0.01 \%$ cresyl violet in $1 \mathrm{~B}$ solution. Then, the sections were washed three times for 1 minute in 1B solution, followed by 3 minutes in $100 \%$ ethanol, 10 minutes in isopropanol, and twice for 5 minutes in xylene before being embedded in a Roti®Histokit mounting medium. Then a cover slip was added to each slide. The solutions required for cresyl violet staining and the staining protocol are listed in the tables 2.11 and 2.12.

Table 2. 11: Solutions required for cresyl violet staining

\begin{tabular}{|c|c|}
\hline Solution & Description \\
\hline Buffer stock solution (1A) & 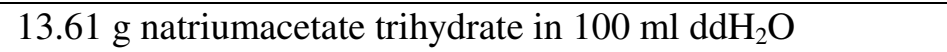 \\
\hline Buffer work solution (1B) & 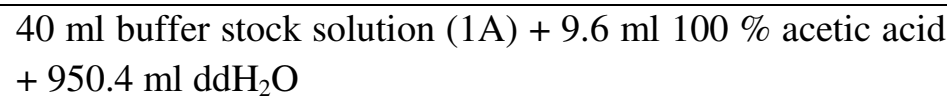 \\
\hline Cresyl violet staining solution (2) & $\begin{array}{l}0.1 \mathrm{~g} \text { cresyl violet in } 11 \mathrm{buffer} \text { working solution (1B). Stir } \\
\text { for } 30 \mathrm{~min} \text {. Let rest overnight. Filter right before use. }\end{array}$ \\
\hline Delipidation stock solution (3A) & 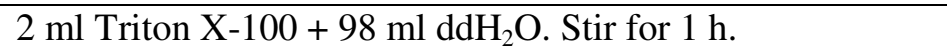 \\
\hline Dilipidation work solution (3B) & 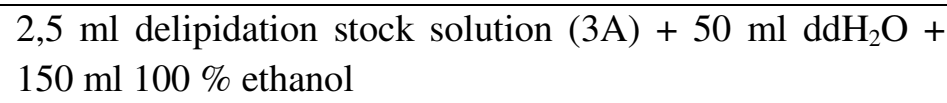 \\
\hline
\end{tabular}

Table 2. 12: Cresyl violet staining protocol

\begin{tabular}{|c|c|}
\hline Step & Description \\
\hline 1. Delipidation & $\begin{array}{l}2 \times 10 \text { min in buffer work solution (1B) } \\
20 \text { min in delipidation work solution (3B) } \\
2 \times 10 \text { min in buffer work solution (1B) }\end{array}$ \\
\hline 2. Staining & $2 \times 8$ min in cresyl violet solution (2) \\
\hline 3. Dehydration & $\begin{array}{l}3 \times 1 \text { min in buffer working solution (1B) } \\
3 \text { min in } 100 \% \text { ethanol } \\
10 \text { min in isopropanol }\end{array}$ \\
\hline 4. Embedding & $\begin{array}{l}2 \text { x } 5 \text { min in xylene } \\
2 \text { drops of xylene-based mounting medium Roti®Histokit. } \\
\text { A cover glass should be firmly pressed on top of glass slide, } \\
\text { carefully pushing away the air bubbles when necessary. }\end{array}$ \\
\hline
\end{tabular}

\subsubsection{Immunostainings}

\subsubsection{DAB immunohistochemistry of paraffin embedded section}

For visualising the $A \beta$ depositions and axonal spheroids, diaminobenzidin (DAB) immunohistochemistry was used.

The glass slides with the dried, paraffin embedded sections were deparaffinized twice for 5 minutes in xylene and rehydrated in baths of decreasing ethanol concentrations (10 
minutes in 100\% ethanol, 5 minutes in 95\% ethanol, 1 minute in $70 \%$ ethanol, 1 minute in $\mathrm{ddH}_{2} \mathrm{O}$ ). Endogenous peroxidases were blocked for 30 minutes in $0,3 \%$ (v/v) hydrogen peroxide in $0.01 \mathrm{M}$ PBS and antigen retrieval was carried out by boiling the sections for 10 min in $0.01 \mathrm{M}$ citrate buffer ( $\mathrm{pH}$ 6.0) in a microwave (to expose the epitopes) after which the sections were left for 15 minutes at RT to cool down. Membrane permeabilization was achieved by washing three times for 5 minutes in $0.01 \mathrm{M}$ PBS containing $0.1 \%$ (v/v) Triton $\mathrm{X}-100$, followed by antigen retrieval in $88 \%$ formic acid for 3 minutes (only for $\mathrm{A} \beta$ staining, to improve the visualization of the intracellular species of $A \beta$ ). Then the sections were circled with a lipid pen. Unspecific binding sites were blocked by one hour treatment with $4 \%(\mathrm{w} / \mathrm{v})$ low-fat dried milk powder and 10\% (v/v) fetal calf serum (FCS) in $0.01 \mathrm{M}$ PBS. Afterwards, the blocking solution was removed (without extra washing) and the sections were incubated overnight in a humid chamber at room temperature with primary antibodies of desired concentration diluted in $0.01 \mathrm{M}$ PBS containing $10 \%(\mathrm{v} / \mathrm{v})$ FCS. The next day the sections were washed three times for 5 minutes in $0.01 \mathrm{M} \mathrm{PBS}$ containing $0.1 \%(\mathrm{v} / \mathrm{v})$ Triton X-100 and were incubated with secondary antibodies at $37^{\circ} \mathrm{C}$ for 1 hour. After washing, the sections were incubated for 1 hour in the respective biotinylated secondary antibody. The secondary antibody was diluted in 0.01 M PBS containing 10\% FCS to the desired concentration (see table 2.16). Then the sections were washed three times for 5 minutes in $0.01 \mathrm{M}$ PBS and incubated 90 minutes at $37^{\circ} \mathrm{C}$ in Avidin-biotin complex (ABC) solution containing 0.01 M PBS, 10\% (v/v) FCS and solutions A (1:100) and B (1:100) from the Vectastain Elite ABC kit (Vector Laboratories, Burlingame, CA, USA) (further "Vectastain Kit") using the ABC method. This solution was prepared according to the instructions of the manufacturer at least 30 minutes before use and stored at $4{ }^{\circ} \mathrm{C}$. After incubation in the $\mathrm{ABC}$-solution the slides were washed three times for 5 minutes with $0.01 \mathrm{M}$ PBS in order to remove the ABC solution.

The staining was finally visualized by 3,3'-Diaminobenzidine (DAB) as chromogen providing reddish-brown colour. The DAB stock solution consisted of $25 \mathrm{mg} / \mathrm{ml} \mathrm{DAB}$ in $50 \mathrm{mM}$ Tris/ $\mathrm{HCl}(\mathrm{pH} 7.5)$. The sections were incubated for up to 3 minutes in the $\mathrm{DAB}$ staining solution, which consisted of $100 \mu \mathrm{DAB}$ stock solution, $5 \mathrm{ml} 50 \mathrm{mM}$ Tris/ $\mathrm{HCl}$, and $2.5 \mu \mathrm{l} 30 \% \mathrm{H}_{2} \mathrm{O}_{2}$ added immediately before use. Afterwards, the sections were washed three times for 5 minutes in $0.01 \mathrm{M}$ PBS. The DAB staining was counterstained with hematoxylin for 40 seconds and put for 5 minutes under running tap water. Thereafter, the sections were dehydrated in baths of increasing ethanol concentrations ( 1 minute in $70 \%$ ethanol, 5 minutes in $95 \%$ ethanol, and 10 minutes in 100\% ethanol) and xylene (two times for 5 minutes). This was followed by embedding in Roti ${ }^{\circledR}$ Histokit mounting medium before a cover glass was firmly pressed on each slide.

For the $\mathrm{A} \beta$ plaque load quantification (see 2.5), the sections were not counterstained in order to avoid prominent background.

Solutions required for DAB immunohistochemistry and the DAB-Immunostaining protocol for paraffin-embedded sections are listed in the tables 2.13 and 2.14.

The antibodies used for this immunostaining are listed in 2.4.2.3. 
Table 2. 13: Solutions required for DAB immunohistochemistry

\begin{tabular}{|l|l|}
\hline Solution & Description \\
\hline 0,01 M PBS solution & Concentrate (PBS 10x Dulbecco's) diluted in $\mathrm{ddH}_{2} \mathrm{O}$ \\
\hline $\begin{array}{l}0,01 \text { M citrate buffer }(\mathrm{pH} \\
6.0)\end{array}$ & $\begin{array}{l}2.1 \mathrm{~g} \text { citric acid monohydrate per liter of } \mathrm{ddH}_{2} \mathrm{O} \text {. The pH was ad- } \\
\text { justed by } \mathrm{NaOH} .\end{array}$ \\
\hline
\end{tabular}

Table 2. 14: DAB-Immunostaining protocol for paraffin-embedded sections on glass

\begin{tabular}{|c|c|}
\hline Step & Description \\
\hline 1. Deparaffinization & $2 \times 5 \mathrm{~min}$ in xylene \\
\hline 2. Hydration & $\begin{array}{l}\text { Baths of decreasing concentrations of ethanol: } \\
10 \min 100 \% \text { ethanol } \\
5 \min 95 \% \text { ethanol } \\
1 \min 70 \% \text { ethanol } \\
1 \min \mathrm{ddH}_{2} \mathrm{O}\end{array}$ \\
\hline 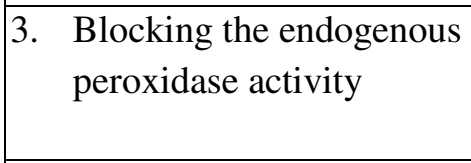 & $\begin{array}{l}30 \text { minutes-incubation in } 0,3 \%(\mathrm{v} / \mathrm{v}) \mathrm{H}_{2} \mathrm{O}_{2} \text { in } 0.01 \mathrm{M} \text { PBS }(200 \mathrm{ml} \\
0.01 \mathrm{M} \mathrm{PBS}+2 \mathrm{ml} 30 \%(\mathrm{v} / \mathrm{v}) \mathrm{H}_{2} \mathrm{O}_{2} \\
1 \mathrm{~min} \mathrm{ddH}_{2} \mathrm{O}\end{array}$ \\
\hline 4. Antigen retrieval & $\begin{array}{l}\text { To expose the epitopes: } \\
\text { Treating sections in the microwave at } 800 \mathrm{~W} \text { in } 0.01 \mathrm{M} \text { citrate buff- } \\
\text { er ( } \mathrm{pH} 6.0 \text { ) for } 2-3 \mathrm{~min} \text { until boiling, then heating at } 80 \mathrm{~W} \text { for } 8 \\
\text { min. } \\
15 \text { min cool down at room temperature }\end{array}$ \\
\hline $\begin{array}{l}\text { 5. Washing and cell mem- } \\
\text { branes permeabilisation }\end{array}$ & $\begin{array}{l}1 \min \mathrm{ddH}_{2} \mathrm{O} \\
15 \min 0.01 \mathrm{M} \mathrm{PBS}+0.1 \%(\mathrm{v} / \mathrm{v}) \text { Triton } \mathrm{X}-100\end{array}$ \\
\hline $\begin{array}{l}\text { 6. Unmasking intracellular } \\
\mathrm{A} \beta \text { epitopes }\end{array}$ & $\begin{array}{l}3 \text { min incubation in } 88 \% \text { formic acid (to improve the visualization } \\
\text { of the intracellular species of } A \beta \text { ) } \\
1 \text { min } 0.01 \mathrm{M} \text { PBS }\end{array}$ \\
\hline 7. Washing & $5 \min 0.01 \mathrm{M}$ PBS \\
\hline $\begin{array}{l}\text { 8. Blocking unspecific } \\
\text { binding }\end{array}$ & $\begin{array}{l}1 \text { hour incubation at RT in solution made of } 0.01 \mathrm{M} \mathrm{PBS}, 10 \%(\mathrm{v} / \mathrm{v}) \\
\text { FCS, and } 4 \%(\mathrm{w} / \mathrm{v}) \text { low-fat dried milk powder. Incubation by cir- } \\
\text { cling sections on glass with lipid pen. }\end{array}$ \\
\hline $\begin{array}{l}\text { 9. Primary antibody incuba- } \\
\text { tion }\end{array}$ & $\begin{array}{l}\text { After unspecific blocking without washing incubation overnight at } \\
\text { room temperature with the antibody in a } 0.01 \mathrm{M} \mathrm{PBS} / 10 \%(\mathrm{v} / \mathrm{v}) \\
\text { FCS solution. }\end{array}$ \\
\hline 10. Washing & $\begin{array}{l}\text { The day after, } 3 \text { x } 5 \text { min washing in } 0.01 \mathrm{M} \mathrm{PBS}+0.1 \%(\mathrm{v} / \mathrm{v}) \text { Tri- } \\
\text { ton X-100 }\end{array}$ \\
\hline $\begin{array}{l}\text { 11. Secondary antibody } \\
\text { incubation }\end{array}$ & $\begin{array}{l}1 \text { hour incubation in the corresponding biotinylated secondary anti- } \\
\text { body diluted in } 0.01 \mathrm{M} \mathrm{PBS} / 10 \%(\mathrm{v} / \mathrm{v}) \mathrm{FCS} \text {, at } 37^{\circ} \mathrm{C} \text {. }\end{array}$ \\
\hline 12. Washing & $3 \times 5 \mathrm{~min}$ in $0.01 \mathrm{M} \mathrm{PBS}$ \\
\hline 13. $\mathrm{ABC}$ incubation & $\begin{array}{l}90 \mathrm{~min} \text { incubation at } 37^{\circ} \mathrm{C} \text { in } 0.01 \mathrm{M} \mathrm{PBS}+10 \%(\mathrm{v} / \mathrm{v}) \mathrm{FCS}+\mathrm{Solu}- \\
\text { tion A }(1: 100)+\text { Solution B }(1: 100) \text { from the Vectastain Kit. Solu- } \\
\text { tion was prepared at least } 30 \mathrm{~min} \text { before use and stored at } 4^{\circ} \mathrm{C} \text {. }\end{array}$ \\
\hline 14. Washing & $3 \times 5 \mathrm{~min}$ in $0.01 \mathrm{M}$ PBS \\
\hline 15. DAB-Staining & $\begin{array}{l}\text { Incubation in } 0.5 \mathrm{mg} / \mathrm{ml} \mathrm{DAB} \text { in } 50 \mathrm{mM} \text { Tris/ } \mathrm{HCl}(\mathrm{pH} 7.5 \text { ) for a few } \\
\text { minutes: } 5 \mathrm{ml} 50 \mathrm{mM} \text { Tris/ } \mathrm{HCl}(\mathrm{pH} 7.5)+100 \mu \mathrm{l} \text { DAB stock solu- } \\
\text { tion }+2.5 \mu \mathrm{l} 30 \% \mathrm{H}_{2} \mathrm{O}_{2} \text { (added just before use). The evolution of }\end{array}$ \\
\hline
\end{tabular}




\begin{tabular}{|l|l|}
\hline Step & Description \\
\hline & $\begin{array}{l}\text { the staining intensity was checked under a microscope and the reac- } \\
\text { tion was stopped by putting the slides in a bath of 0.01 M PBS. }\end{array}$ \\
\hline 16. Washing & $3 \times 5$ min in $0.01 \mathrm{M}$ PBS \\
\hline 17. Counterstaining & $40 \mathrm{~s}$ incubation in hematoxylin \\
& $\begin{array}{l}1 \text { min in in } \mathrm{ddH}_{2} \mathrm{O} \\
5 \text { min under running tap water } \\
1 \text { min } \mathrm{ddH}_{2} \mathrm{O}\end{array}$ \\
\hline 18. Dehydration & Baths of increasing ethanol concentrations. \\
& 1 min $70 \%$ ethanol \\
& 5 min $95 \%$ ethanol \\
& 10 min $100 \%$ ethanol \\
\hline 19. Embedding & $2 \times 5$ min xylene \\
& Embedding in a xylene-based mounting medium Roti®Histokit. A \\
& cover glass should be firmly pressed on top of glass slide, carefully \\
& pushing away the air bubbles when necessary. \\
\hline
\end{tabular}

\subsubsection{Free-floating immunohistochemistry}

The embedding procedure and the harsh treatments (for example high temperatures) adversely affect some epitopes. That is why immunostainings in paraffin sections are inapplicable for detection of antigens with sensitive epitopes. Nevertheless, in most cases those antigens can be visualized by immunostaining in free-floating frozen sections. That was the case for the neurogenesis marker doublecortin (DCX) for which a specific protocol has been applied. The following DAB-Immunostaining protocol for free-floating frozen sections was taken up from (Cotel 2009) and modified.

In this study, DCX was used to visualize new-born neurons and reflect neurogenesis in Tg4-42 $2_{\text {hom }}$ mice (Figure 2.2). DCX is a microtubule-associated protein that is expressed in all migrating neuronal precursors of the developing central nervous system (CouillardDespres et al. 2005). Initially, doublecortin positive neurones were found in the subventricular zone where new neurons start migration to the olfactory bulbs, and in the subgranular zone of the dentate gyrus (Lledo et al. 2006) (see 1.5.5).

The brain treatment was done as described above in 2.3. The frozen section series comprising every tenth section throughout the brain (one section series from each animal) were rehydrated for 10 minutes in $4^{\circ} \mathrm{C} 0.01 \mathrm{M}$ PBS solution in order to remove organic glue. The cold $0.01 \mathrm{M}$ PBS was used to avoid breaks in the integrity of the slices due to heat-shock. Then the sections were transferred into Netwells ${ }^{\mathrm{TM}}$ using cold 0.01 M PBS as transfer liquid (all sections from each glass container were put in separate Netwells ${ }^{\mathrm{TM}}$ ). All of the following incubation and washing steps were carried out in Netwells ${ }^{\mathrm{TM}}$ (Figure 2.3) on a rotating plate. 


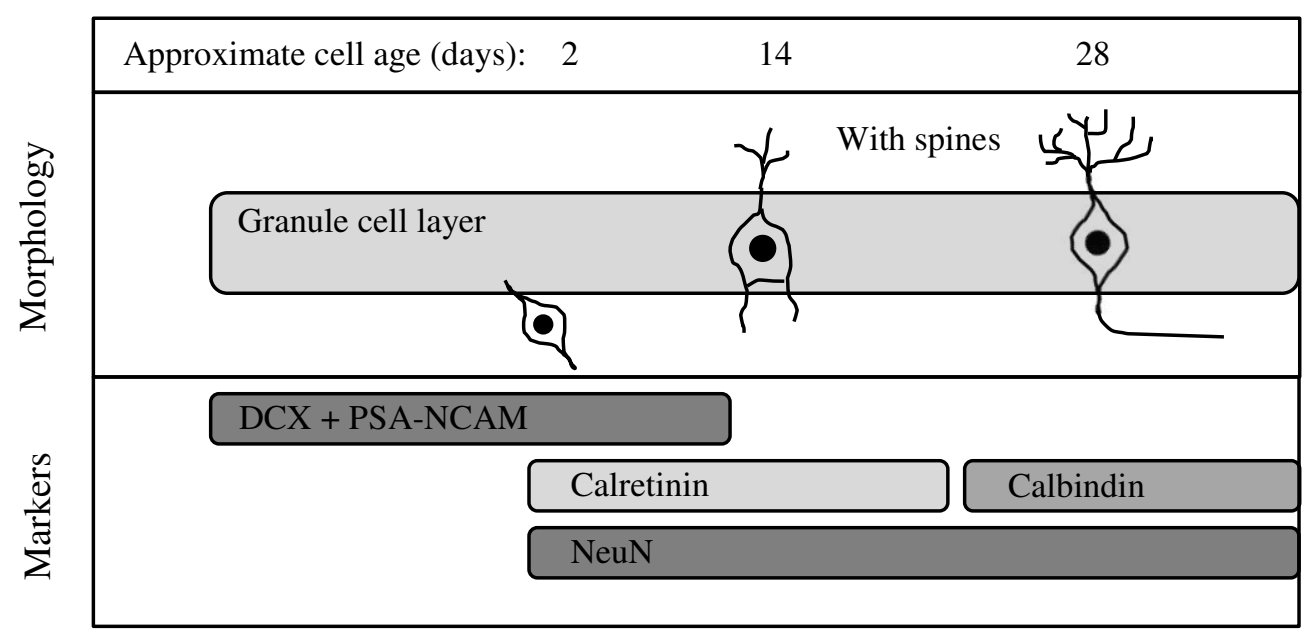

Figure 2. 2: Hippocampal granule cells expressing different markers. Abbreviations: DCX, doublecortin; GAD, glutamate decarboxylase; NeuN, neuronal nuclear antigen; PSA-NCAM, polysialic acid-neuronal cell adhesion molecule. Figure created after Lledo et al. 2006, p. 181.

At first, the sections were quenched for 30 minutes in a solution of $0.01 \mathrm{M}$ PBS with $0,3 \%$ (v/v) $\mathrm{H}_{2} \mathrm{O}_{2}$ for the purpose of blocking endogenous peroxidase activity. The sections were subsequently washed three times for 10 minutes in $0.01 \mathrm{M} \mathrm{PBS} / 0.1 \%(\mathrm{v} / \mathrm{v})$ Triton X100 followed by incubation for one hour at room temperature in a solution of $4 \%(\mathrm{w} / \mathrm{v})$ low-fat dried milk powder and 10\% (v/v) FCS in 0.01 M PBS in order to block unspecific binding sites. Then the blocking solution was removed and the primary antibody (DCX) diluted (1:500) in $0.01 \mathrm{M}$ PBS containing $10 \%$ (v/v) FCS was added into Netwells ${ }^{\circledR}$. The sections were incubated overnight at room temperature. The next day the sections were washed three times for 10 minutes in 0.01 M PBS containing $0.1 \%(\mathrm{v} / \mathrm{v})$ Triton X-100 and incubated with a secondary antibody diluted as above at room temperature for two hours. Then the sections were washed three times for 10 minutes in 0.01 M PBS and incubated for 90 minutes at RT in a Avidin-biotin complex (ABC) solution from the Vectastain Kit using the ABC method (see 2.4.2.1).

After washing the staining was visualized by 3,3'-Diaminobenzidine. The DAB staining solution was made as described in 2.4.2.1. The sections were incubated for 5 minutes in the DAB staining solution. Afterwards, the sections were washed three times for 10 minutes in $0.01 \mathrm{M}$ PBS.

After washing, stained sections were mounted onto glass slides, then carefully unfolded with a thin brush and left dry overnight at RT.

The next day the sections were counterstained with hematoxylin. This was done as follows. At first, the glass slides were put for 10 minutes in 0.01 M PBS for hydration. Then the slides were counterstained with hematoxylin for 40 seconds, dipped shortly in $\mathrm{ddH}_{2} \mathrm{O}$ and put for 5 minutes under running tap water. Thereafter, the sections were dehydrated in baths of increasing ethanol concentrations as described in 2.4.2.1 and subsequently embedded in Roti ${ }^{\circledR}$ Histokit mounting medium before a cover slip was added to each slide.

The DAB-Immunostaining protocol for free-floating frozen sections is recapitulated in table 2.15. 
The antibodies used for this immunostaining are listed in 2.4.2.3.

Table 2. 15: DAB-Immunostaining protocol for free-floating frozen sections

\begin{tabular}{|c|c|}
\hline Step & Description \\
\hline $\begin{array}{l}\text { 1. Organic glue removal } \\
\text { and rehydration }\end{array}$ & $\begin{array}{l}10 \text { min in } 0.01 \mathrm{M} \text { cold } 0.01 \mathrm{M} \text { PBS in which the sections are moved } \\
\text { into Netwells }{ }^{\mathrm{TM}} \text {. } \\
10 \text { min } 0.01 \mathrm{M} \text { PBS for hydration. }\end{array}$ \\
\hline $\begin{array}{l}\text { 2. Blocking the endogenous } \\
\text { peroxidase activity }\end{array}$ & $\begin{array}{l}30 \text { minutes-incubation in } 0,3 \%(\mathrm{v} / \mathrm{v}) \mathrm{H}_{2} \mathrm{O}_{2} \text { in } 0.01 \mathrm{M} \text { PBS }(200 \mathrm{ml} \\
0.01 \mathrm{M} \text { PBS }+2 \mathrm{ml} 30 \%(\mathrm{v} / \mathrm{v}) \mathrm{H}_{2} \mathrm{O}_{2}\end{array}$ \\
\hline 3. Washing & $\begin{array}{l}3 \times 10 \min \text { in } 0.01 \mathrm{M} \text { PBS }+0.1 \%(\mathrm{v} / \mathrm{v}) \text { Triton } \mathrm{X}-100 \\
1 \mathrm{~min} \text { in } 0.01 \mathrm{M} \mathrm{PBS}\end{array}$ \\
\hline $\begin{array}{l}\text { 4. Blocking unspecific } \\
\text { binding }\end{array}$ & $\begin{array}{l}1 \text { hour incubation at room temperature in solution made of } 0.01 \mathrm{M} \\
\mathrm{PBS}, 10 \%(\mathrm{v} / \mathrm{v}) \mathrm{FCS} \text {, and } 4 \%(\mathrm{w} / \mathrm{v}) \text { low-fat dried milk powder. }\end{array}$ \\
\hline $\begin{array}{l}\text { 5. Primary antibody incuba- } \\
\text { tion }\end{array}$ & $\begin{array}{l}\text { After unspecific blocking, without washing, incubation overnight at } \\
\text { room temperature with the antibody in a } 0.01 \mathrm{M} \mathrm{PBS} / 10 \%(\mathrm{v} / \mathrm{v}) \\
\text { FCS solution. }\end{array}$ \\
\hline 6. Washing & $3 \times 10 \mathrm{~min}$ in $0.01 \mathrm{M}$ PBS \\
\hline 7. $\mathrm{ABC}$ incubation & $\begin{array}{l}90 \mathrm{~min} \text { incubation at } 37^{\circ} \mathrm{C} \text { in } 0.01 \mathrm{M} \mathrm{PBS}+10 \%(\mathrm{v} / \mathrm{v}) \mathrm{FCS}+\text { Solu- } \\
\text { tion A }(1: 200)+\text { Solution B }(1: 200) \text { from the Vectastain Kit. Solu- } \\
\text { tion was prepared at least } 30 \mathrm{~min} \text { before use and stored at } 4^{\circ} \mathrm{C} \text {. }\end{array}$ \\
\hline 8. Washing & $3 \times 10 \mathrm{~min}$ in $0.01 \mathrm{M}$ PBS \\
\hline 9. $\quad$ DAB-Staining & $\begin{array}{l}\text { Incubation in } 0.5 \mathrm{mg} / \mathrm{ml} \mathrm{DAB} \text { in } 50 \mathrm{mM} \text { Tris } / \mathrm{HCl}(\mathrm{pH} \text { 7.5) for } 5 \\
\text { min: } 5 \mathrm{ml} 50 \mathrm{mM} \text { Tris } / \mathrm{HCl}(\mathrm{pH} 7.5)+100 \mu \mathrm{DAB} \text { stock solution }+ \\
2.5 \mu 130 \% \mathrm{H}_{2} \mathrm{O}_{2} \text { (added just before use). The reaction was stopped } \\
\text { by plunging the slides in a bath of } 0.01 \mathrm{M} \text { PBS. }\end{array}$ \\
\hline 10. Washing & $3 \times 10 \mathrm{~min}$ in $0.01 \mathrm{M}$ PBS \\
\hline 11. Mounting & $\begin{array}{l}\text { Sections were mounted in } 0.01 \mathrm{M} \text { PBS onto glass slides and left to } \\
\text { dry overnight. }\end{array}$ \\
\hline 12. Counterstaining & $\begin{array}{l}10 \text { min in } 0.01 \mathrm{M} \text { PBS for hydration } \\
40 \mathrm{~s} \text { incubation in hematoxylin } \\
1 \mathrm{~min} \text { in in } \mathrm{ddH}_{2} \mathrm{O} \\
5 \text { min under running tap water }\end{array}$ \\
\hline 13. Dehydration & $\begin{array}{l}\text { Baths of increasing ethanol concentrations. } \\
1 \text { min } 70 \% \text { ethanol } \\
5 \text { min } 95 \% \text { ethanol } \\
10 \text { min } 100 \% \text { ethanol }\end{array}$ \\
\hline 14. Embedding & $\begin{array}{l}2 \times 5 \text { min xylene } \\
\text { Embedding in a xylene-based mounting medium Roti®Histokit, } \\
\text { one drop per section. A cover glass should be firmly pressed on top } \\
\text { of glass slide, carefully pushing away the air bubbles when neces- } \\
\text { sary. }\end{array}$ \\
\hline
\end{tabular}




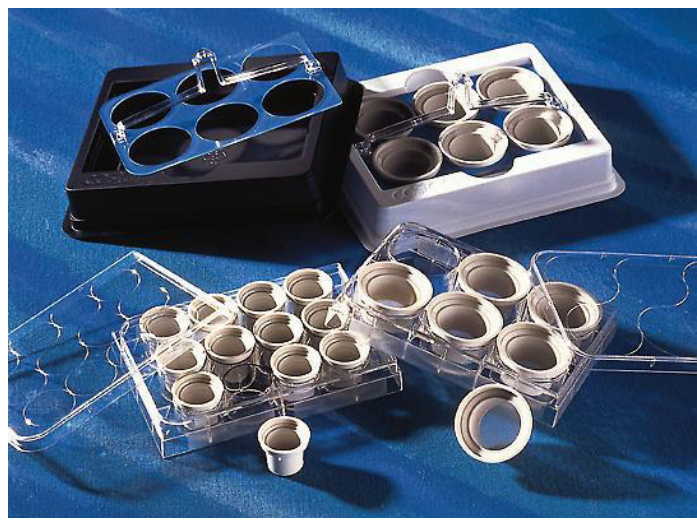

Figure 2. 3: Netwells ${ }^{\mathrm{TM}}$. $15 \mathrm{~mm}$ Netwell ${ }^{\mathrm{TM}}$ Insert with $440 \mu \mathrm{m}$ Mesh Size Polyester Membrane. Reproduced from http://www.corning.com

\subsubsection{Antibodies}

The following tables $(2.16,2.17)$ show the primary and secondary antibodies, their host organisms and corresponding dillutions.

Table 2. 16: Primary antibodies used for immunohistochemical stainings

\begin{tabular}{|c|c|c|c|c|}
\hline Antibody & $\begin{array}{l}\text { Physiological } \\
\text { target }\end{array}$ & Host & Dilution & Manufacturer \\
\hline $\mathrm{A} \beta[\mathrm{N}]$ & $A \beta 1-x$ & $\begin{array}{l}\text { Polyclonal Rab- } \\
\text { bit }\end{array}$ & $1: 500$ & $\begin{array}{l}\text { IBL, Hamburg, } \\
\text { Germany }\end{array}$ \\
\hline $\begin{array}{l}\text { Anti- } \\
\text { Neurofilament-L } \\
\# 171002\end{array}$ & Neurofilament-L & $\begin{array}{l}\text { Monoclonal, } \\
\text { Mouse }\end{array}$ & $1: 500$ & $\begin{array}{l}\text { Synaptic Sys- } \\
\text { tems, Göttingen }\end{array}$ \\
\hline DCX & $\begin{array}{l}\text { Doublecortin, } \\
\text { expressed by } \\
\text { migrating neu- } \\
\text { rons }\end{array}$ & $\begin{array}{l}\text { Polyclonal, } \\
\text { Goat }\end{array}$ & $1: 500$ & $\begin{array}{l}\text { Santa Cruz } \\
\text { Biotechnology, } \\
\text { Inc., USA }\end{array}$ \\
\hline
\end{tabular}

Table 2. 17: Secondary antibodies applied for immunohistochemistry

\begin{tabular}{|l|l|l|}
\hline Antibody & Dilution & Manufacturer \\
\hline Rabbit anti-mouse immunoglobulins, biotinylated, E0465 & $1: 200$ & DAKO, Denmark \\
\hline Swine anti-rabbit immunoglobulins, biotinylated, E0353 & $1: 200$ & DAKO, Denmark \\
\hline Rabbit anti-goat immunoglobulins, biotinylated, E0466 & $1: 250$ & DAKO, Denmark \\
\hline
\end{tabular}




\subsection{Quantification of neuron number by stereology}

\subsubsection{Design-based stereology}

Design-based stereology is an unbiased stereological method. Different quantities can be explored with stereology in order to make quantitative statements regarding function of tissues and organs. For example: the number of things such as number of brain cells within a brain region can be counted; length such as the length of axons can be measured; volume such as volume of the brain region; surface areas of the shape that is not flat such as the surface of a membrane can be estimated (West 2012).

Due to the stereology, it is possible to describe 3D structures from measurements that are made on 2D images (West 2012). Design-based stereology (further "Stereology") avoids all assumptions about the shape, size, spatial orientation or spatial distribution of the object of study, but uses carefully randomized probes (e.g. brain sections) for an unbiased estimation of different quantities. All sections are investigated with a uniform random probability. The results obtained with stereology can be easily reproduced. Another advantage of this method is efficiency. It allows estimating the number of cells of a brain region with a very low error margin using only few systematically sampled sections (Schmitz and Hof 2005; West 2002)

\subsubsection{Optical dissector}

The optical fractionator is the central tool of Stereology. It allows sampling a 3D region of interest and estimating the total number of particles (i.e. neurons in our case) (West et al. 1991). The optical fractionator method uses the optical dissector. The optical dissector is a $2 \mathrm{D}$ counting frame projected along the $\mathrm{Z}$-axis.

The counting frame is usually shown as a red and green probe. Green and red lines are inclusion and exclusion lines, respectively (see figure 2.4). The third dimension is the $Z$ dimension, the so-called disector height.

The positions of counting frames are determined by randomly placing a rectangular grid on the surface of a section (figure 2.5).

\subsubsection{Counting rules and criteria for counting cells}

During the counting, the set of rules must be followed in order to achieve unbiased estimation (Gundersen 1977; West et al. 1991). At first, the cell's unique point must be identified. In this study, the nucleus' top was used as the unique point. The unique point must fall within the disector height. The counting frame rules are applied when the unique point first comes into the focus. Cells should not be counted if the unique point comes into the focus while in the guard zone. 
Guard zones are regions at the top and bottom of the tissue section in which cells must not be marked (figure 2.6). They are needed, because some cells can be damaged, cut or plucked from the tissue through the cutting prozess. Guard zones help to exclude them from counting.

A
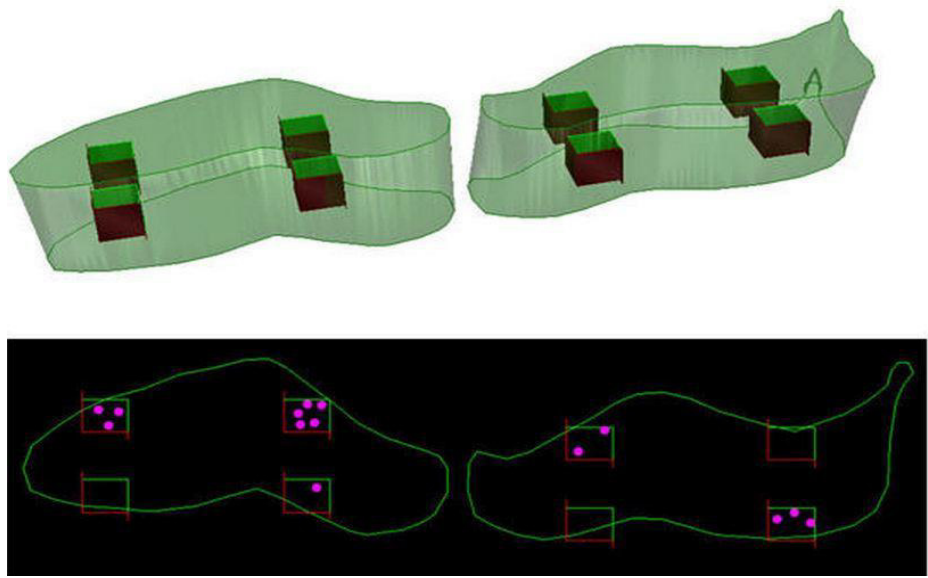

Figure 2. 4: The optical fractionator. The optical fractionator probe uses thick sections to estimate the total number of cells from the number of cells sampled (pink dots in (B)). (A)Three-dimensional representation of randomly placed 3D counting frames. (B) An optical disector is limited by the intersectional red and green lines. Reproduced from http://www.stereology.info/the-optical-fractionator/

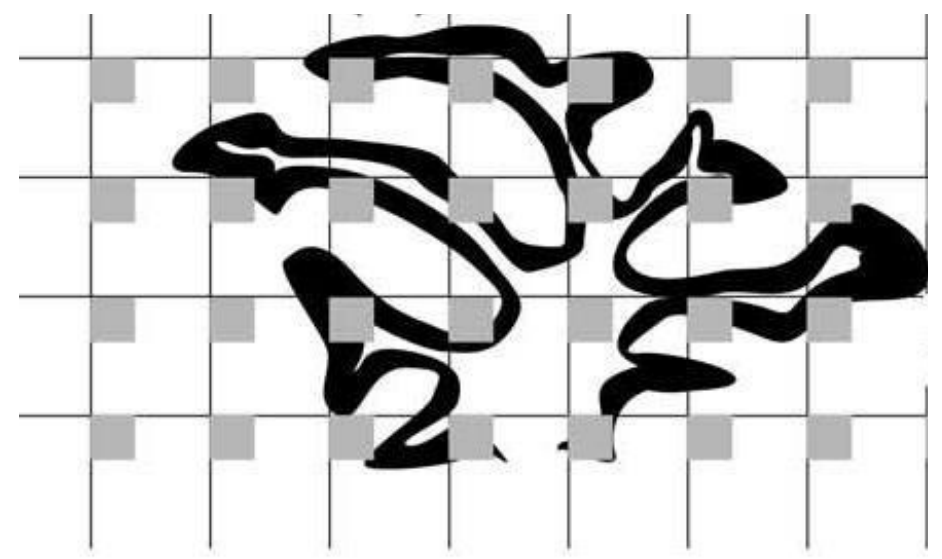

Figure 2. 5: Sampling grid. A randomly placed rectangular grid (big white squares) with side lengths $x$ and $y$ on the surface of a section, determining the positions of unbiased counting spaces (small grey squares). Figure modified from Schmitz and Hof 2005, p. 818.

A marker should be placed on the unique point, when the unique point first comes into focus. If any area of it touches the red exclusion line or touches/crosses both an inclusion line and an exclusion line, the cell must not be counted.

Figure 2.7 demonstrates how counting frame rules guarantee that cells are counted once and only once. 


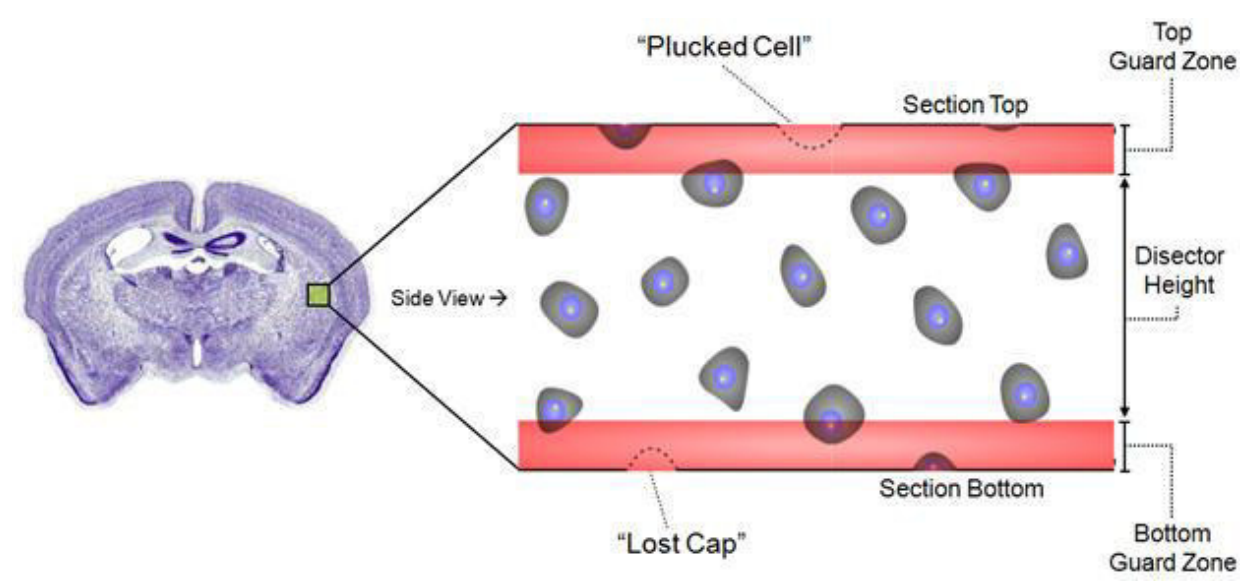

Figure 2. 6: Guard zones. Explaining the necessity to set them in order to have a homogenous sample. Reproduced from www.mbfbioscience.com

A

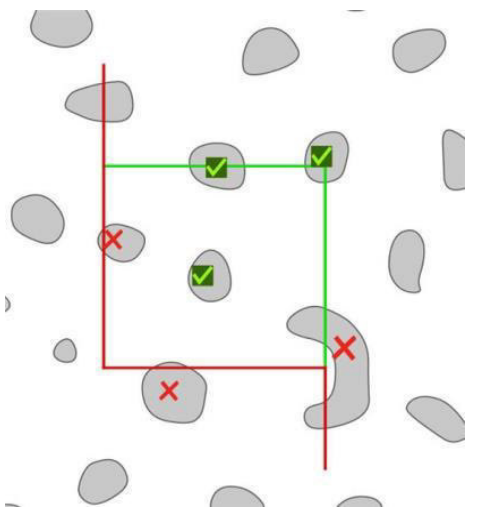

B

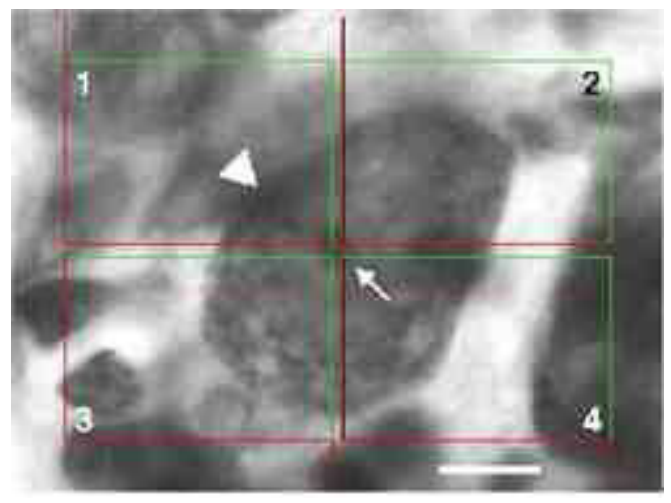

Figure 2. 7: Counting rules. (A) An example of using a counting frame to include or exclude objects: a cell is counted if it lies completely inside the counting frame or if it crosses a green line but not a red line. The grey shapes represent the unique points. (B) Four counting frames positioned very close to each other. The Purkinje cell appears in all counting frames, but it is only counted for frame 3 , since it crosses the exclusion lines in frames 1,2 , and 4.

(A) and (B) are reproduced from http://www.stereology.info/criteria-for-counting-cells/ and http://www.stereology.info/counting-rules/ respectively.

\subsubsection{Quantification of the total neuron number in the granular cell layer of the dentate gyrus}

For the estimation of the total granule cell number in the dentate gyrus of $\mathrm{Tg} 4-42_{\mathrm{hom}}$ mice, the design-based stereology (Schmitz and Hof 2005; West et al. 1991) (see 2.3.1) was applied.

The tissue for quantification was prepared as described in 2.3. The left hemispheres were cut frontally into entire series of $30-\mu \mathrm{m}$ thick sections; every tenth section was systematically sampled and stained with cresyl violet (see 2.4.1). 
The stereological analysis was performed as previously described (Casas et al. 2004; Schmitz et al. 2004). The sampling grid area ( $x y)$, counting frame area $(X Y)$ and disector height $(Z)$ were adapted to the evaluated region (granular cell layer of the dentate gyrus) (see table 2.18).

The granular cell layer of the dentate gyrus was delineated at low magnification (40X) on cresyl violet-stained sections (Bregma -1.34 to -3.80 (Paxinos and Franklin 2001)) (see figure 2.8). Using a stereology workstation equipped with an Olympus BX-51 microscope with a motorized specimen stage for automatic sampling (StereoInvestigator 7, MicroBrightField, Williston, VT, USA), the neuronal nuclei were sampled at high magnification (1000X) as described in 2.5.1, and the total number of neurons was subsequently estimated by the fractional method using a $2-\mu \mathrm{m}$ top guard zone. The section thickness was measured on every grid site with a dissector height $(Z)$ of $5 \mu \mathrm{m}$. On average, 10 sections per animal were used to estimate the total neuron number of the granular cell layer of the dentate gyrus. In order to guarantee unbiased counting, all samples were blinded. The following numbers of animals were used in this analysis: $\mathrm{Tg} 4-42_{\mathrm{hom}}: n=11(6 \mathrm{~m}, \mathrm{EE}), n=8(6 \mathrm{~m}$, SH), WT: $n=3(6 \mathrm{~m}, \mathrm{SH})$.

The number of cells in the region of interest was estimated by the following formula (Mouton 2002; Schmitz and Hof 2005; West et al. 1991, S. 199):

$$
N=\sum_{i=1}^{n} Q_{i}^{-} \times \frac{1}{t s f} \times \frac{1}{a s f} \times \frac{1}{s s f}
$$

Where:

- $\quad N$ : estimated total number of neurons in the dentate gyrus

- $\quad \sum_{i=1}^{n} Q_{i}^{-}$: the sum of neuron numbers of all analysed sections in one animal, where $Q_{i}$ are the total numbers of counted objects in each section $i$ of one animal.

- $t s f:$ thickness sampling fraction ( $t s f=\frac{Z}{T}$, where $Z$ and $T$ are the disector height and the section thickness, respectively)

- asf: area sampling fraction ( $a s f=\frac{x y}{X Y}$, where $x y$ and $X Y$ are a sampling grid area and a counting frame area, respectively)

- $\quad s s f$ : section sampling fraction (in our case $s s f=10$, because every tenth section was analysed.

The stereologic parameters' values used for the estimation of the DG are listed in the table 2.18. 

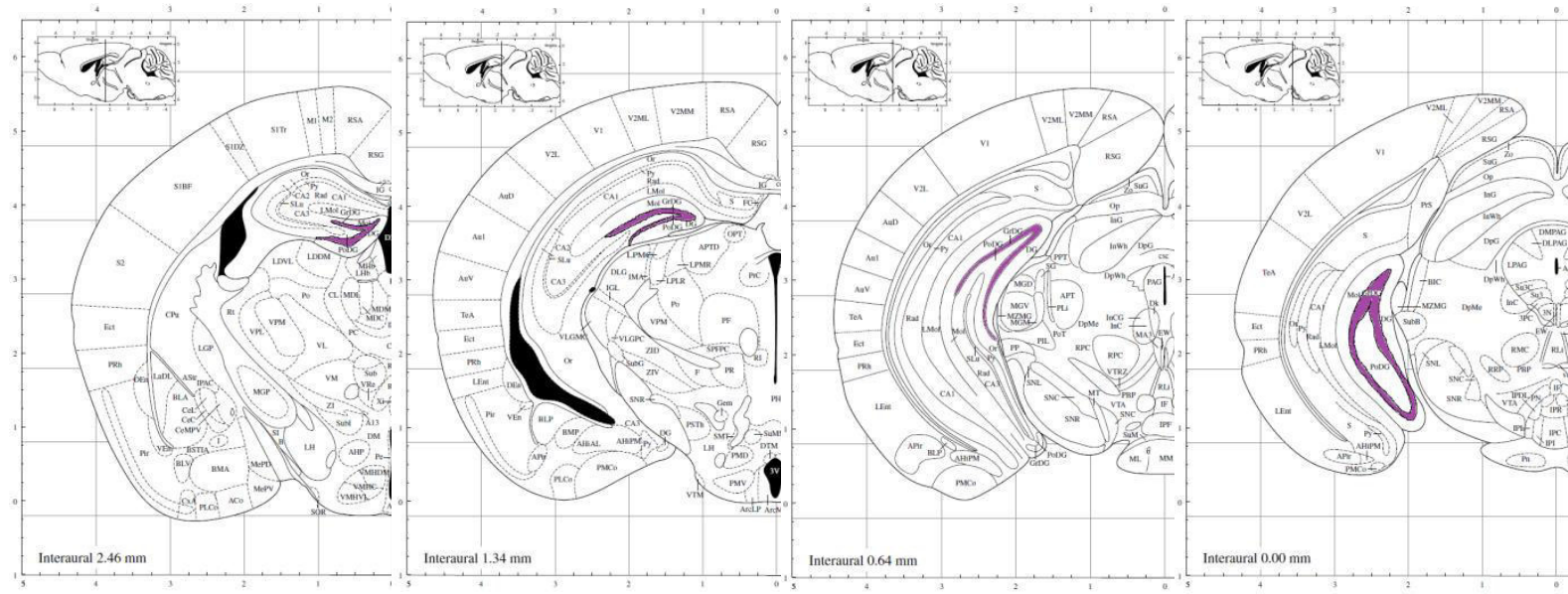

Figure 2. 8: Coronal slices of the mouse brain highlighting the dentate gyrus (adapted from Paxinos and Franklin 2001). DG is set off in violet ink. From left to right: Bregma $-1.22,-2.46,-3.16$ and $-3.80 \mathrm{~mm}$.

Table 2. 18: Parameters for stereological analysis of the granular cell layer of the dentate gyrus

\begin{tabular}{|l|c|}
\hline Paramete & DG \\
\hline Disector Height $(\mathrm{Z})(\mu \mathrm{m})$ & 5 \\
\hline Disector Volume $(\mathrm{XYZ})\left(\mu \mathrm{m}^{3}\right)$ & 980 \\
\hline Sampling Grid $(\mathrm{x})(\mu \mathrm{m})$ & 133 \\
\hline Sampling Grid $(\mathrm{y})(\mu \mathrm{m})$ & 75 \\
\hline Sampling Grid Area $(\mathrm{xy})\left(\mu \mathrm{m}^{2}\right)$ & 9975 \\
\hline Counting Frame Width $(\mathrm{X})(\mu \mathrm{m})$ & 14 \\
\hline Counting Frame Height $(\mathrm{Y})(\mu \mathrm{m})$ & 14 \\
\hline Counting Frame Area $(\mathrm{XY})\left(\mu \mathrm{m}^{2}\right)$ & 196 \\
\hline T $(\mu \mathrm{m})$ [average value] & 8.87 \\
\hline $\mathrm{Z}(\mu \mathrm{m})$ & 5 \\
\hline tsf & 0,6 \\
\hline asf & 50.9 \\
\hline ssf & 10 \\
\hline
\end{tabular}

\subsection{Estimation of the volume of the dentate gyrus}

To estimate the volume of the dentate gyrus, Cavalieri's principle was used according to (Rosen and Harry 1990). Cavalieri's principle is an unbiased estimator, which is based on a rectangular approximation of the area under a curve and requires systematic sampling (Cruz-Orive 1985; Gundersen and Jensen 1987). This principle was claimed to be an efficient tool for volume estimation of irregular brain regions (Uylings et al. 1986). Rosen and Harry (1990) confirmed this in their study. They suggested the use of Cavalieri's principle in all cases of volume estimation of regularly and irregularly shaped, equally spaced brain regions. When only a few sections are available, it provides the best estimate of the volume. Cavalieri's principle is demonstrated in the figure 2.9. 
The mathematical formula used to calculate the volume is the following:

$$
V_{C}=d \times\left(\sum_{i=1}^{n} y_{i}\right)-t \times y_{M A X}
$$

Where:

- $V_{C}$ : estimated volume

- $d$ : distance between the sections that were analysed (in this case $\mathrm{d}=300 \mu \mathrm{m}$ as we analyse every tenth $30 \mu \mathrm{m}$-thick section)

- $\quad y_{i}$ : cross-sectional area of the $i$-th section through the morphometric region ${ }^{4}$.

- $n$ : total number of sections

- $y_{M A X}$ : maximal value of y (maximum area)

- $t$ : corresponding thickness of the $y_{M A X}$ section (Gundersen and Jensen 1987)

The product of $y_{M A X}$ and $t$ is subtracted from the basic equation as a correction for overprojection. "Correction for overprojection is required when section thickness is not negligible, but the volume of the project is large" (Rosen and Harry 1990)

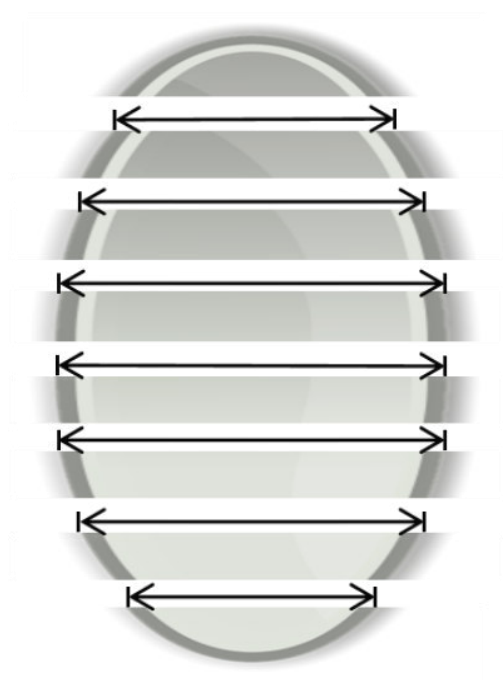

$\mathbf{A}$

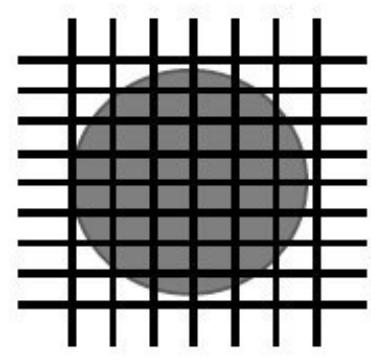

B

Figure 2. 9: Cavalieri's principle for the estimation of the volume of a brain region. This principle is applied for volume estimation of regularly and irregularly shaped, equally spaced brain regions. (A) The brain region is cut into series of sections of uniform thickness, and the area of every $\mathrm{x}$-th section is measured (arrows at the upper edge). An unbiased estimate of the volume of the brain region is obtained by multiplying the sum of its areas on all analysed sections with the uniform section thickness (Schmitz and Hof 2005). (B) The area of each section is measured by point counting method; in a square array test system the point area is calculated as the square of the distance between two points (Mandarim-de-Lacerda 2003, p. 475). The points are randomly but systematically distributed by a software program. Figure (A) created after Schmitz and Hof 2005 , p. 819.

\footnotetext{
${ }^{4}$ Cross-sectional area of each section was estimated with StereoInvestigator 7 during the estimation of total neuron number of the dentate gyrus.
} 


\subsection{Quantification of $A \beta$ plaque load}

The extracellular $A \beta$ load in the 5XFAD mice was evaluated in the cortex, the hippocampus, and the thalamus (figure 2.10) with an Olympus BX-51 microscope equipped with a Moticam Pro 282A camera (Motic) and the ImageJ software (V1.41, NIH, USA). A $\beta[N]$, an antibody marking all $A \beta$ species, was chosen for this analysis. The sagittal brain sections of $4 \mu \mathrm{m}$ thickness were immunostained using DAB as a chromogene (see 2.4.2.1). Serial images of $100 \mathrm{X}$ or $200 \mathrm{X}$ magnification were captured on an average of four to six sections per animal, which were at least $30 \mu \mathrm{m}$ afar from each other. Using ImageJ the pictures were binarized to 8-bit black and white images and a fixed intensity threshold was applied defining the DAB stained area. The area fraction covered by DAB staining was estimated.
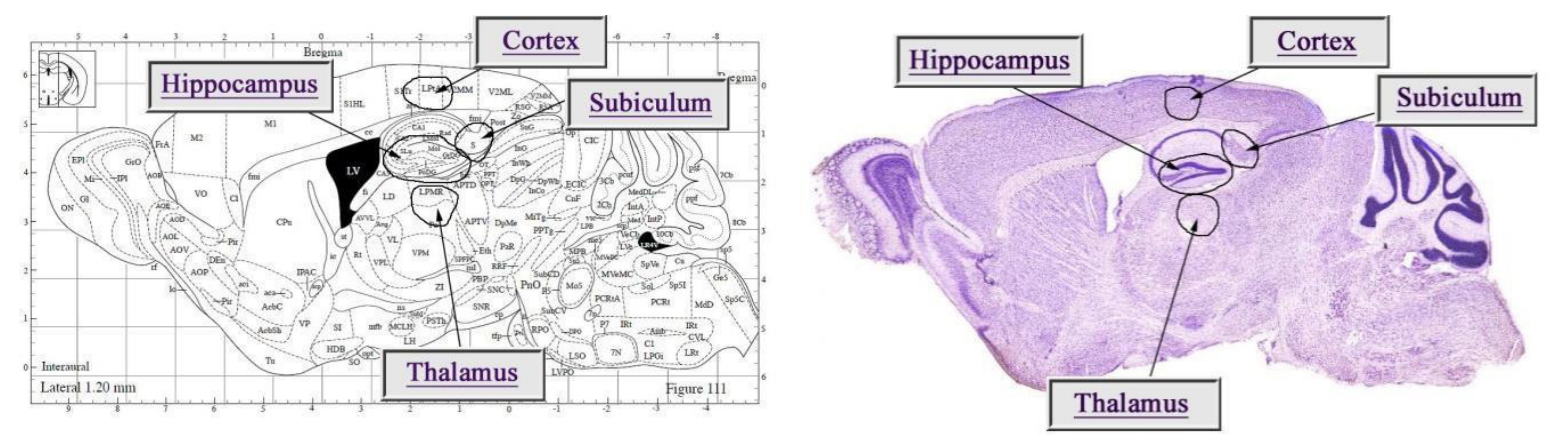

Figure 2. 10: Brain regions, in which the extracellular $A \beta$ load was evaluated. Adapted from Paxinos and Franklin 2001.

For the statistical analysis, the mean values of the measurements in each animal were used. For the parts of the hippocampal formation such as the subiculum and the hippocampal region, ellipsoid frames of constant size adjusted to the desired brain region were created with ImageJ before quantification. The area outside of the ellipsoid was not included in the analysis (Figure 2.11). The protocol for $\mathrm{A} \beta$ plaque load quantification used in this study was elaborated by my colleague Bernhard Richard.

For better comparison, the relative $\mathrm{A} \beta$ load is expressed with $5 \mathrm{XFAD}_{\text {het }}$ mice as the reference parameter. The following number of animals was used in this analysis: 5XFAD hem: $n=6(2 \mathrm{~m}), n=8(5 \mathrm{~m}), n=3-5(9 \mathrm{~m})$; 5XFAD hom $: n=4(2 \mathrm{~m}), n=7(5 \mathrm{~m}), n=$ 3-4 (9 m). One-way ANOVA followed by unpaired $t$-tests was used to compare age dependent changes in plaque load for each antibody (Richard et al. 2015). 


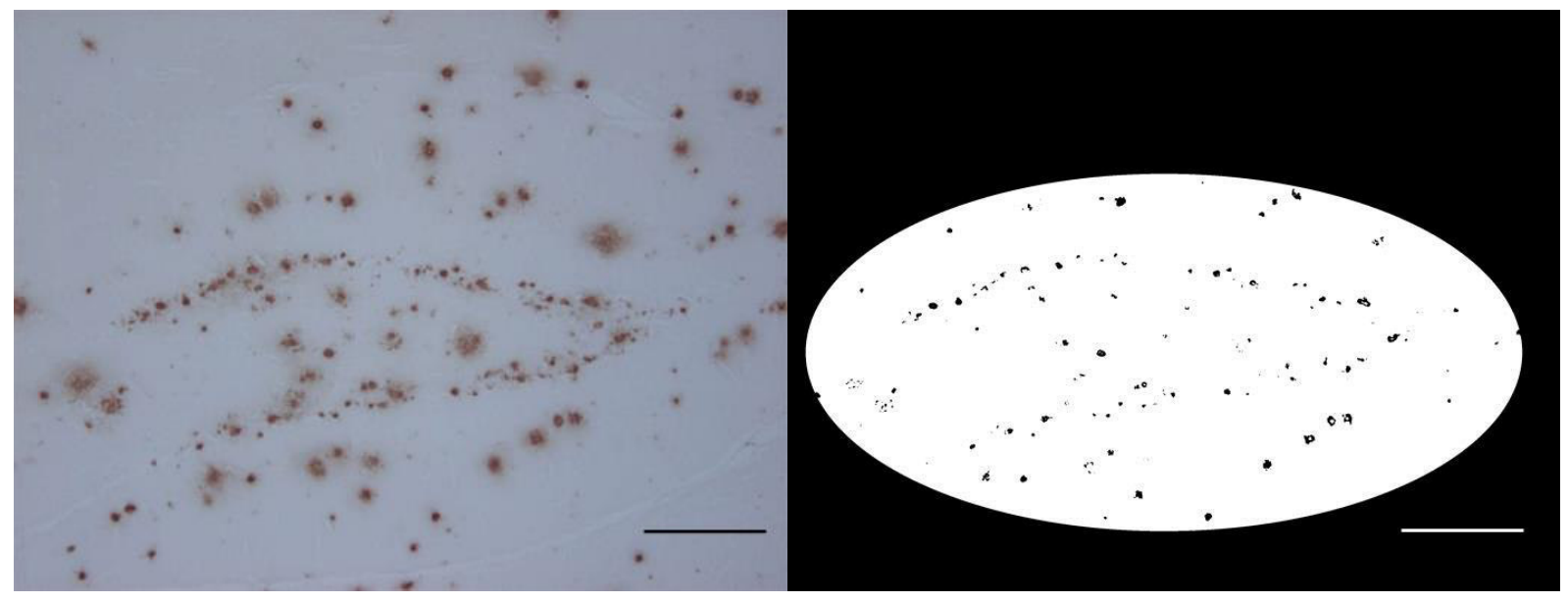

Figure 2. 11: An example of the application of the ellipsoid frame in the plaque load quantification. Hippocampus of a 5 -month-old $5 \mathrm{XFAD}_{\text {hom }}$ mouse. The initial image is on the left side; the image after application of the frame is on the right side. Scale bar: $200 \mu \mathrm{m}$. Source: own research.

\subsection{Quantification of doublecortin-positive neurons and plaque independent neurofilament-positive axonal spheroids}

The number of DCX-positive neurons within the subgranular zone (SGZ) of the dentate gyrus was quantified in order to estimate the adult neurogenesis in $\mathrm{Tg} 4-42_{\text {hom }}$ mice. The sections used for the estimation of adult neurogenesis were obtained by systematically collecting every tenth coronal frozen section of $30 \mu \mathrm{m}$-thickness (see 2.3 and 2.4.2.2). The number of DCX-positive neurons was estimated by multiplying the number of markers by a factor 10. The following number of animals was used in this analysis: Tg4-42hom: $n=11$ (6 m, EE), $n=8(6 \mathrm{~m}, \mathrm{SH}), \mathrm{WT}: n=5(6 \mathrm{~m}, \mathrm{SH})$. The same Tg4-42 $2_{\mathrm{hom}}$ mice were used for the estimation of the total neuron number of the dentate gyrus (see 2.5).

To estimate the axonal degeneration, paraffin sections of the brain and the spinal cord from 9-month-old animals were double-stained for neurofilament-L (see 2.4.2.1) and the number of the neurofilament-L-positive axonal spheroids was quantified. The estimation was performed on an average of four to six $4 \mu \mathrm{m}$-thick sections per animal which were at least $30 \mu \mathrm{m}$ apart from each other (see 2.3). The following number of animals was used in this analysis: $5 \mathrm{XFAD}_{\text {hem }}: n=3$ (spinal cord), $n=5$ (pons), $5 \mathrm{XFAD}_{\text {hom }}: n=4$ (both spinal cord and the pons). The dystrophic neurites that presented a diameter greater than $5 \mu \mathrm{m}$ and that were not located in the immediate vicinity of amyloid plaques were counted as axonal spheroids.

The DCX-positiv neurons and neurofilament-L-positive axonal spheroids were counted using the Meander Scan platform of StereoInvestigator 7 (MBF Bioscience, Williston, VT, USA) at 1000X and 200X magnification. 


\subsection{Statistical analyses, software and images}

The statistical analysis of the collected data was performed with GraphPad PRISM 5.01 for Windows (GraphPad Software, Inc., San Diego, CA, USA). The differences between the groups were tested with a one-way analysis of variance (ANOVA) followed by unpaired t-tests. All data are presented as mean \pm s.e.m. The significance levels were given as follows: $* * * p<0.001 ; * * p<0.01 ; * p<0.05$.

DNA in the gel after agarose gel electrophoresis was analysed with the Quantity One software program (Version 4.30; Biorad).

The qRT-PCR data were collected using the MxPro Mx3000P software (Stratagene, Santa Clara, CA, USA).

Stereo Investigator 7 software (MBF Bioscience, Williston, VT, USA) was used for design-based stereology.

The images of the stained tissue were acquired using a BX-51 microscope (Olympus, Shinjuku, Japan) equipped with with a Moticam Pro 282A camera (Motic).

The images for the plaque load quantification were processed with the ImageJ software (V1.41, NIH, USA).

Graphs were plotted with GraphPad PRISM. Figures were composed with Adobe Photoshop CS2 (Adobe Systems, San Jose, CA, USA). 


\subsection{Lists of chemicals, reagents, kits and technical devices}

The following tables list the chemicals (table 2.19), reagents and kits (table 2.20) and technical devices (table 2.21), which were used in this study.

Table 2. 19: Chemicals

\begin{tabular}{|l|l|}
\hline Chemical & Manufacturer \\
\hline 3,3'-Diaminobenzidin (DAB) & Roth, Karlsruhe, Germany \\
\hline Acetic acid & Merck, Darmstadt, Germany \\
\hline Agarose & Lonza, Basel, Switzerland \\
\hline Boric acid & Sigma, St. Louis, MO, USA \\
\hline Citric acid & Roth, Karlsruhe, Germany \\
\hline Cresyl violet & Fluka, St. Louis, MO, USA \\
\hline Ethanol & Merck, Darmstadt, Germany \\
\hline Ethidium bromide & Roth, Karlsruhe, Germany \\
\hline Ethylenediaminetetraacetic acid (EDTA) & Roth, Karlsruhe, Germany \\
\hline Formic acid $98 \%$ & Roth, Karlsruhe, Germany \\
\hline Hydrochloric acid (HCl) & Merck, Darmstadt, Germany \\
\hline Hydrogen peroxide ( ${ }_{2} \mathrm{O}_{2}$ ) & Roth, Karlsruhe, Germany \\
\hline Isopropanol & Roth, Karlsruhe, Germany \\
\hline Molecular grade water & Braun, Melsungen, Germany \\
\hline Natriumacetate trihydrate & Roth, Karlsruhe, Germany \\
\hline Paraformaldehyde (PFA) & Roth, Karlsruhe, Germany \\
\hline Sodium chloride (NaCl) & Roth, Karlsruhe, Germany \\
\hline Sodium dodecyl sulfate (SDS) & Roth, Karlsruhe, Germany \\
\hline Sucrose & Roth, Karlsruhe, Germany \\
\hline Tris (hydroxymethyl) aminomethane (Tris) & Roth, Karlsruhe, Germany \\
\hline Triton X-100 & Roth, Karlsruhe, Germany \\
\hline Xylene & Roth, Karlsruhe, Germany \\
\hline
\end{tabular}

Table 2. 20: Reagents and kits

\begin{tabular}{|l|l|}
\hline Reagent/Kit & Manufacturer \\
\hline 100 bp Ladder & Bioron, Ludwigshafen, Germany \\
\hline 10x reaction buffer & Axon, Kaiserslautern, Germany \\
\hline Agarose Sample Buffer (Blue Juice) & Life Technologies, Carlsbad, CA, USA \\
\hline Desoxyribonukleosidetriphosphates (dNTPs) & Axon, Kaiserslautern, Germany \\
\hline Dulbecco's phosphate buffered salt solution (PBS) & PAN-Biotech, Aidenbach, Germany \\
\hline DyNAmo Flash SYBR Green qPCR Kit & Thermo Fisher Scientific, Waltham, MA, USA \\
\hline Fetal Calf Serum & Thermo Fisher Scientific, Waltham, MA, USA \\
\hline Hematoxylin Solution & Roth, Karlsruhe, Germany \\
\hline Ketamine 10 \% & Medistar, Ascheberg, Germany \\
\hline
\end{tabular}




\begin{tabular}{|c|c|}
\hline Reagent/Kit & Manufacturer \\
\hline Magnesium chloride $\left(\mathrm{MgCl}_{2}\right)$ for PCR & Axon, Kaiserslautern, Germany \\
\hline Non-Fat Dry Milk & Roth, Karlsruhe, Germany \\
\hline Paraffin for tissue embedding & Roth, Karlsruhe, Germany \\
\hline Primers for genotyping & Eurofins, Ebersberg, Germany \\
\hline Proteinase $\mathrm{K}$ & Peqlab, Erlangen, Germany \\
\hline Roti®Histokitt & Roth, Karlsruhe, Germany \\
\hline Taq DNA-Polymerase & Axon, Kaiserslautern, Germany \\
\hline $\begin{array}{l}\text { Tissue-Tek }{ }^{\circledR O} \text { O.C.T. }{ }^{\mathrm{TM}} \text { Compound (soluble glycol } \\
\text { and resin compound) }\end{array}$ & SAKURA Finetek Europe \\
\hline Vectastain Elite ABC Kit & Vestor Laboratories, Burlingame, CA, USA \\
\hline Xylazine (Xylariem) & Ecuphar, N.V. Oostkamp, Belgium \\
\hline
\end{tabular}

Table 2. 21: Technical devices

\begin{tabular}{|l|l|}
\hline Device & Manufacturer \\
\hline Biophotometer & Eppendorf, Hamburg, Germany \\
\hline Cenrifuge (Heraeus Biofuge Pico) & Thermo Fisher Scientific, Waltham, MA, USA \\
\hline Centifuge (Heraeus Biofuge Stratos) & Thermo Fisher Scientific, Waltham, MA, USA \\
\hline Centrifuge (Spectrafuge Mini) & Labnet Inc., Edison, NJ, USA \\
\hline CM1850 UV Cryostat & Leica, Wetzlar, Germany \\
\hline EG1140 H Embedding Station & Leica, Wetzlar, Germany \\
\hline Electrophoresis chamber & Biorad Laboratories, Hercules, CA, USA \\
\hline Embedding cassettes & Simport, Beloeil, QC, Canada \\
\hline Eppendorf LoBind reaction tubes & Eppendorf, Hamburg, Germany \\
\hline HM 335E Microtome & MICROM, Germany \\
\hline Individually Ventilated Cages (IVC) & Tecniplast, Hohenpleissberg, Germany \\
\hline LabCycler for PCR & SensoQuest, Goettingen, Germany \\
\hline Microscope cover glasses & Thermo Fisher Scientific, Waltham, MA, USA \\
\hline MX3000P Real-Time Cycler & Stratagene, Santa Clara, CA, USA \\
\hline Netwells ${ }^{\circledR}$ & Corning Inc., Corning, NY \\
\hline Odyssey FC & Li-Cor, Bad Homburg, Germany \\
\hline Pap Pen Lipid Pen & Kisker Biotech, Steinfurt, Germany \\
\hline PCR tubes & Greiner Bio-One, Kremsmünster, Austria \\
\hline PCR tubes (for qRT-PCR) & Biozym, Oldendorf, Germany \\
\hline Power Pack P25 power supply & Biometra, Goettingen, Germany \\
\hline Superfrost ${ }^{\circledR}$ glass slides & Thermo Fisher Scientific, Waltham, MA, USA \\
\hline Thermomixer Compact & Eppendorf, Hamburg, Germany \\
\hline TP 1020 Automatic Tissue Processor & Leica, Wetzlar, Germany \\
\hline Uvette® 220-1600 nm cuvette & Eppendorf, Hamburg, Germany \\
\hline Vortexer (Vortex Genie 2) & Scientific Industries, Bohemia, NY, USA \\
\hline Water bath for mounting of paraffin tissue & Medax, Olching, Germany \\
\hline & \\
\hline
\end{tabular}




\section{RESULTS}

\subsection{Project I: Physical activity ameliorates neuron loss and increases neurogen- esis in 6-month-old Tg4-42 ${ }_{\text {hom }}$ mice.}

\subsubsection{Total neuron number: enriched environment and physical exercise atten-

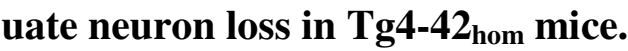

The total neuron number of the granular cell layer of the dentate gyrus was quantified using design-based stereology in cresyl violet stained frozen sections of 6-month-old Tg4$42_{\text {hom }}$ mice (Figure 3.1). The light microscopy revealed more lossened structure of DG in SH Tg4-42 hom mice compared to the DG of EE Tg4-42 $2_{\text {hom }}$ mice. No shrinkage of DG was observed (Figure 3.1, A-D).

A statistically highly significant neuron loss $(\mathrm{p}=0.0002)$ was observed in standard housed 6-month-old $\mathrm{Tg} 4-42_{\text {hom }}$ mice $(446000 \pm 24060, \mathrm{n}=8)$ compared to $\mathrm{Tg} 4-42_{\text {hom }}$ mice that lived under enriched conditions $(591500 \pm 18980, \mathrm{n}=11)$. The difference in the granular cell number between the $\mathrm{SH}$ and the EE group of mice was $+25 \%$. Stereological quantification showed similar numbers of neurons between WT mice $(540000 \pm 43140, n=$ 3 ) and EE Tg4-42 hom mice $(591500 \pm 18980, \mathrm{n}=11)$. The difference in the cell numbers between SH Tg4-42hom and wild-type mice was $-18 \%(\mathrm{p}=0.0773)$. There were highly significant differences between all three groups (one-way ANOVA, $\mathrm{p}=0,0006$ ). Therefore, stereology revealed that enriched hosing conditions ameliorate neuron loss in $\operatorname{Tg} 4-42_{\text {hom }}$ mice and restore the neuron number to the levels of the age-matched wild-types.

The numbers were estimated for the left hemispheres.

\subsubsection{Volume of the dentate gyrus: no significant difference between EE Tg4- 42 $\mathrm{hom}_{\text {, }}$ SH Tg4-42 $\mathrm{hom}$, and WT mice.}

The volume of DG was estimated in order to analyze if physical activity and cognitive stimulation have an impact on it. Despite the massive neuron loss in the DG, the analysis of the volume of the dentate gyrus revealed no significant difference $(p>0.05)$ neither

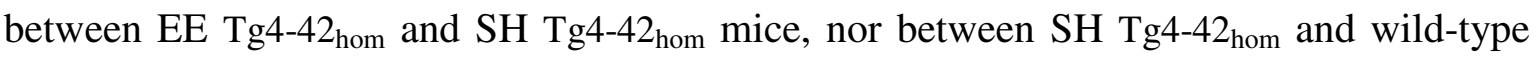
mice (Figure 3.2). 
EE, 6
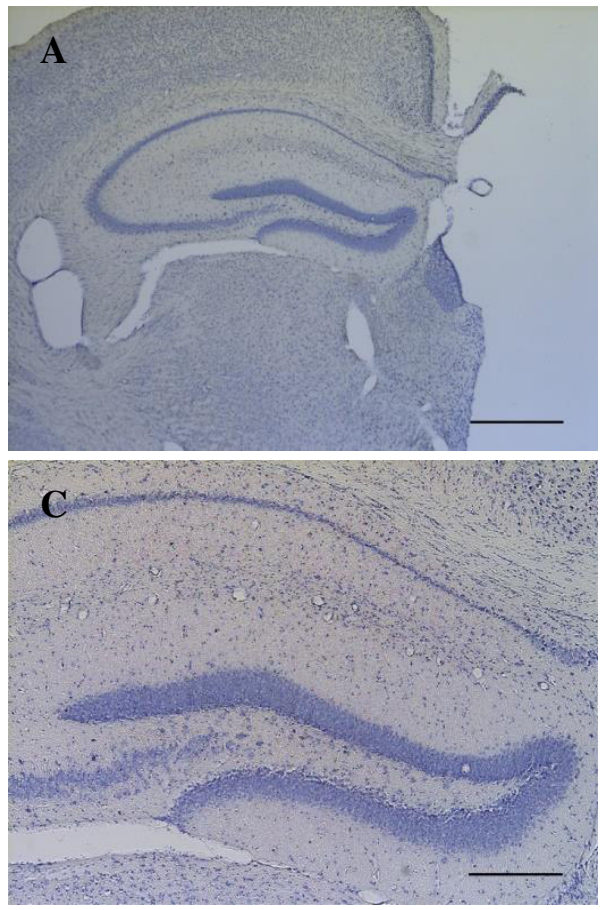

E
SH, 6
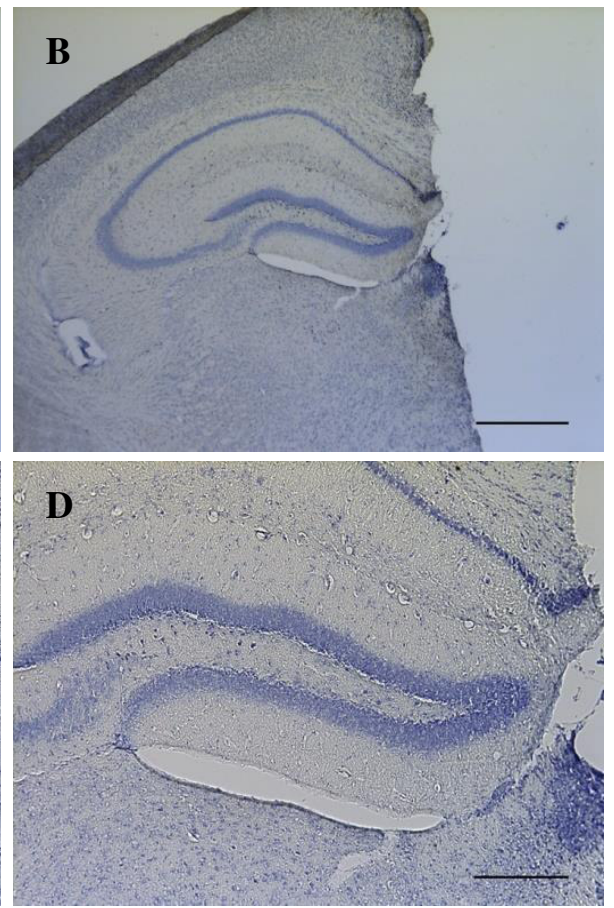

Tg4- $42_{\mathrm{hom}}, 6 \mathrm{~m}, \mathrm{SH}$

$\mathrm{Tg} 4-42_{\text {hom }}, 6 \mathrm{~m}, \mathrm{EE}$

WT, $6 \mathrm{~m}, \mathrm{SH}$

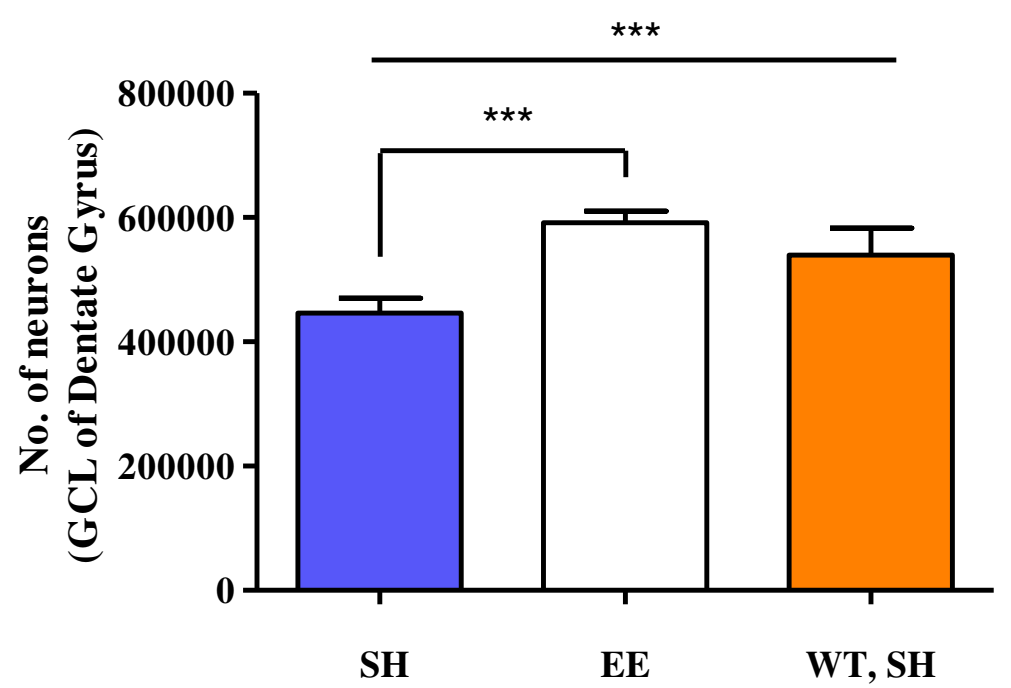

Figure 3. 1: Quantification of neurons in GCL of the dentate gyrus using unbiased stereology. (A-D) Cresyl violet stained coronal frozen sections (approx. Bregma -1.80 according to Paxinos and Franklin (2001)). Loosened structure of the DG in SH Tg4-42 hom in comparison to EE Tg4-42 hom mice. (E) Significant difference in the cell number between SH Tg4-42 hom and EE Tg4-42 hom mice. Data presented as mean \pm s.e.m; $* * * p<0.001 ; \mathrm{m}=$ months. Scale bar (A-B): $500 \mu \mathrm{m}$; scale bar (C-D): $200 \mu \mathrm{m}$. 


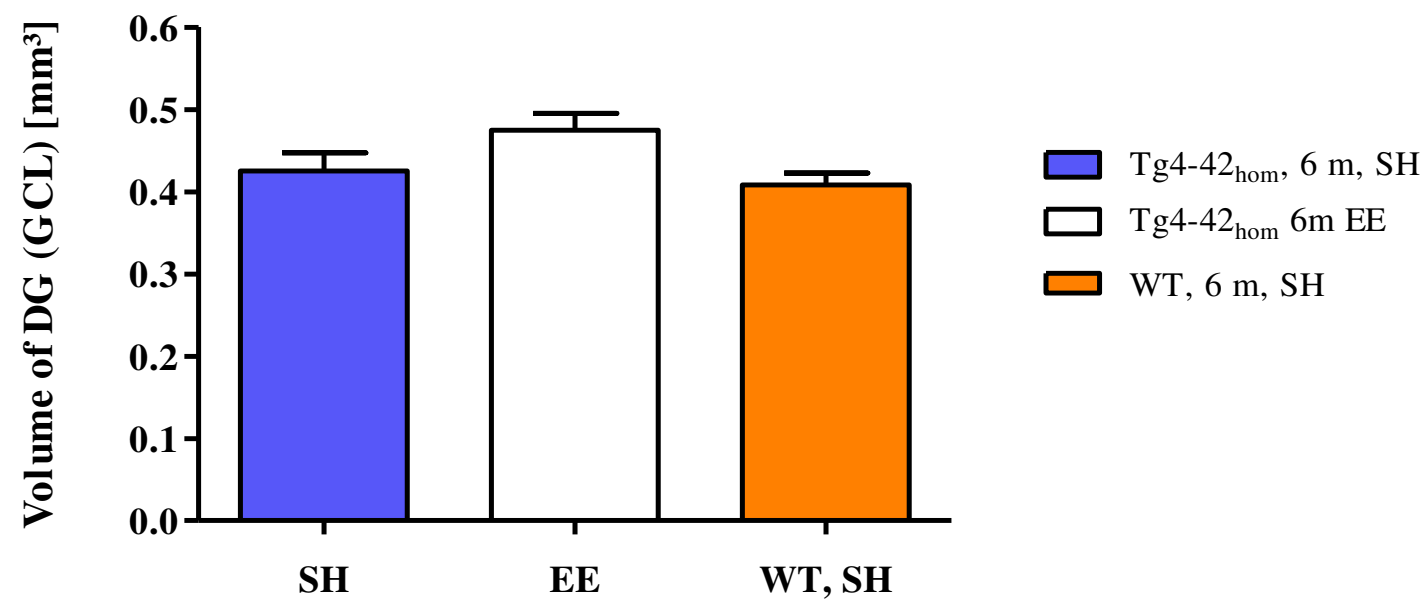

Figure 3. 2 Volume of the GCL of the dentate gyrus. No significant difference between SH Tg4-42 ${ }_{\text {hom }}$ and EE Tg4- $42_{\text {hom }}$ mice could be detected. Data presented as mean \pm s.e.m; $p>0.05 ; \mathrm{m}=$ months.

\subsubsection{Effects of environmental enrichment on adult subgranular neurogenesis: enriched environment triggers neurogenesis in Tg4-42 $\mathrm{hom}$ mice}

In order to quantify the adult subgranular neurogenesis in $\mathrm{Tg} 4-42_{\mathrm{hom}}$ mice and analyze if the enriched environment has an influence on it, free-floating frozen sections of Tg4$42_{\text {hom }}$ mice brains were stained with an antibody against doublecortin (DCX). DCX is a microtubule-associated protein, which is exclusively expressed in immature neurons in the subventricular zone, where new neurons start migration to the olfactory bulbs, and in the subgranular zone of the dentate gyrus (Couillard-Despres et al. 2005; Lledo et al. 2006).

The analysis revealed a significant difference between $\mathrm{Tg} 4-42_{\mathrm{hom}}$ mice that lived under enriched conditions with increased physical activity and environmental diversity (6920 \pm $366.7 ; n=11)$ and standard housed $\mathrm{Tg} 4-42_{\text {hom }}$ mice $(4555 \pm 506.9 ; n=8)$. The neuron number was strongly increased $(+35 \%)$ in EE-housed $\mathrm{Tg} 4-42_{\mathrm{hom}}$ mice compared to $\mathrm{SH}$ Tg4-42 hom mice (Figure 3.3, J). The number of new-born neurons in the SGZ of the DG of SH Tg4-42 $2_{\text {hom }}$ mice $(4555 \pm 506.9 ; n=8)$ was dramatically reduced $(-43 \%)$ in comparison with SH wild-types $(7886 \pm 359.9 ; n=5)$. This decline could be restored by 4 months of housing under EE conditions.

Furthermore, DCX-positive neurons in DG of the standard housed Tg4-42 hom mice showed atrophic, in the DCX-staining almost not visible dendrites in comparison with $\mathrm{SH}$ wild-types. The dendrites of DCX-positive cells of EE housed Tg4-42 shorter and less branched than those of SH wild-types. However, they were more pronounced than the dendrites of standard housed Tg4-42 hom mice (Figure 3.3, A-I).

The results of the Project I have been published in Hüttenrauch et al. 2016. 


\section{Tg4-42 ${ }_{\mathrm{hom}}, 6 \mathrm{~m}, \mathrm{SH}$}

Tg4-42

WT, 6 m, SH

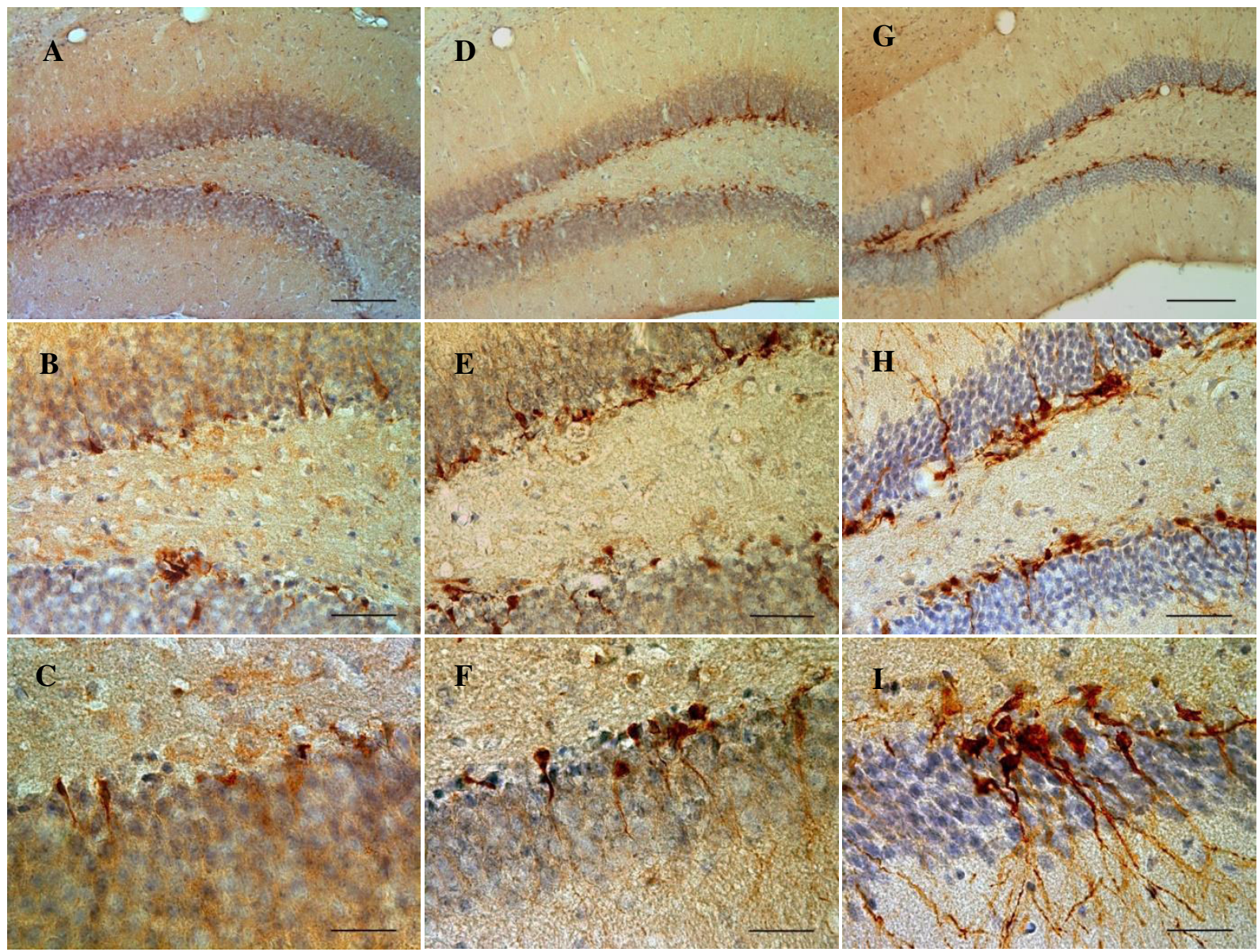

J
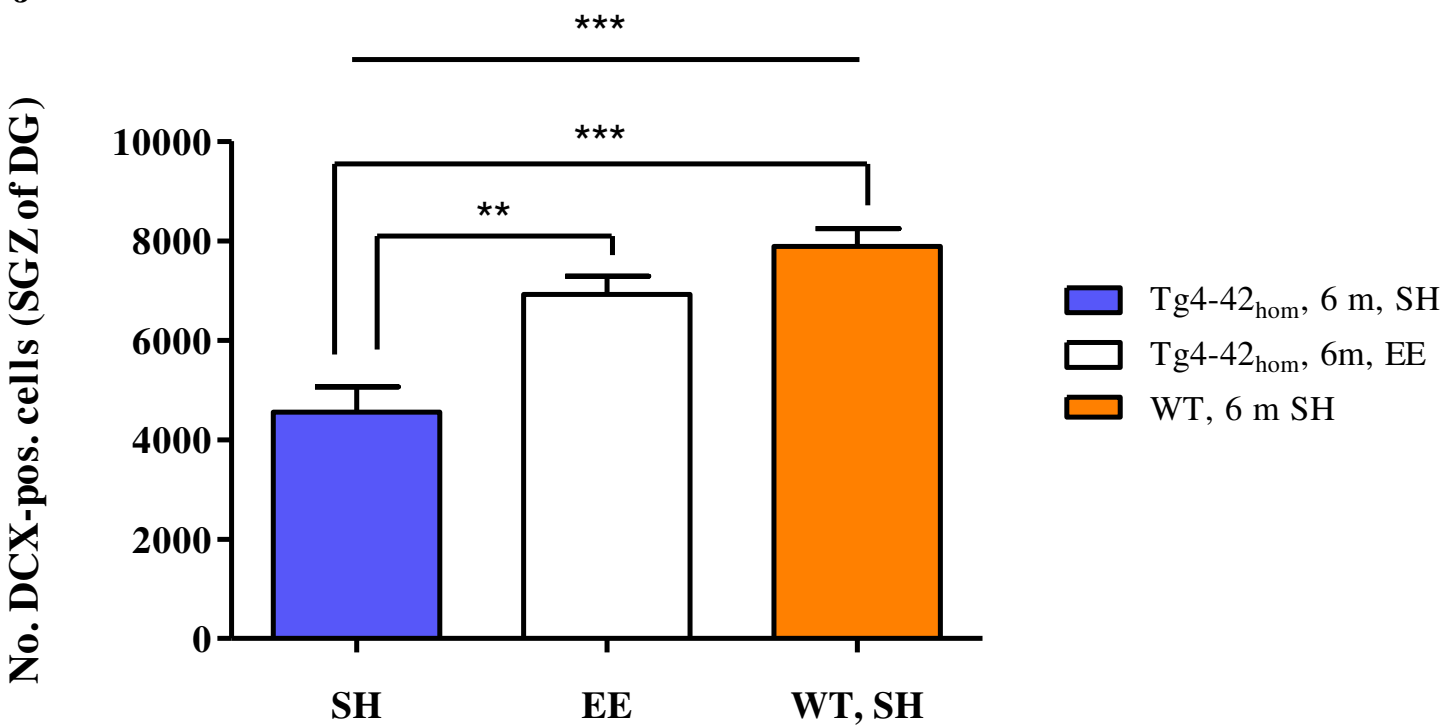

$\square \mathrm{WT}, 6 \mathrm{~m} \mathrm{SH}$

Figure 3. 3: Number of doublecortin-positive neurons in the SGZ of the dentate gyrus. (A-I) Coronal sections of Tg4-42 $\mathrm{hom}$ and WT mice stained with antibody against DCX. (J) The neurogenesis was significantly increased $(+35 \%)$ in EE Tg4-42 hom mice in comparison to SH Tg4-42 hom mice. The number of new-born neurons in the SGZ of the DG of SH Tg4-42 hom mice was dramatically impaired (-43\%) comparing with the WT mice. $\mathrm{m}=$ months. Data presented as mean \pm s.e.m; *** $p<0.001$, ** $p<0.01$. Scale bar $(\mathrm{A}, \mathrm{D}, \mathrm{G}): 100 \mu \mathrm{m}$; scale bar (B, E, H): $33 \mu \mathrm{m}$; scale bar (C, F, I): $20 \mu \mathrm{m}$. 


\subsection{Project II: Gene dosage-dependent increase of amyloid pathology and ax- onal degeneration in $5 X_{F A D}$ mice.}

\subsubsection{Quantification of A $\beta$ plaque load}

To quantify the gene dosage-dependent increase of the $\mathrm{A} \beta$ plaque load in 5XFAD mice, sagittal brain sections of $5 X F A D_{\text {hem }}$ and $5 X X_{\text {hom }}$ mice at 2, 5 and 9 months of age were stained with an $A \beta[N]$ antibody, which detects an $N$-terminal $A \beta$ epitope.

Relative amyloid load was quantified in the cortex, hippocampus (hippocampal region (CA and DG) (further "hippocampus"), retrohippocampal region (subiculum) (further "subiculum")) and the thalamus as described earlier (see 2.7) (Christensen et al. 2008). A significant increase in $\mathrm{A} \beta$ plaque load was found in $5 \mathrm{XFAD}_{\text {hom }}$ compared to $5 \mathrm{XFAD}_{\text {hem }}$ mice in all analysed brain regions at all time points (Cortex: $2 \mathrm{~m}$ : $+232 \%$; $5 \mathrm{~m}$ : $+228 \%$; $9 \mathrm{~m}$ : +32\%; Hippocampus: $2 \mathrm{~m}$ : $+615 \%$; $5 \mathrm{~m}:+464 \%$; $9 \mathrm{~m}:+52 \%$; Subiculum: $2 \mathrm{~m}:+103 \% ; 5$ m: $+66 \%$; $9 \mathrm{~m}: 61 \%$; Thalamus: $2 \mathrm{~m}:+311 \%$; $5 \mathrm{~m}:+101 \%$; $9 \mathrm{~m}:+75 \%$ ) (Figure 3.6). Young mice at 2 months of age showed the highest increase. Aged mice at 9 months of age showed only moderate increases. The increase of the amyloid pathology is illustrated in the figures 3.4 and 3.5.

\section{Cortex}

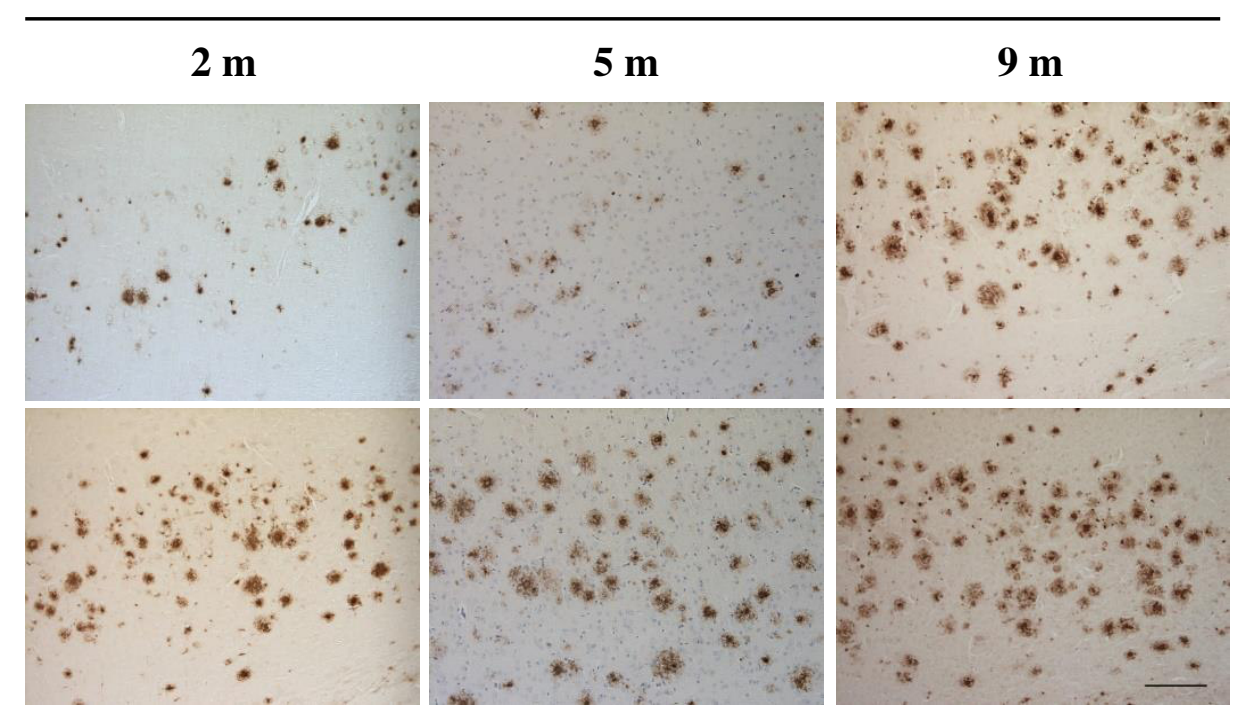

Figure 3. 4: Illustration of extracellular $A \beta$ plaque load in cortex. Strong increase of amyloid load in $5 \mathrm{XFAD}_{\text {hom }}$ mice at all time points compared to $5 \mathrm{XFAD}_{\text {hem }}$. Scale bar: $100 \mu \mathrm{m}$. 


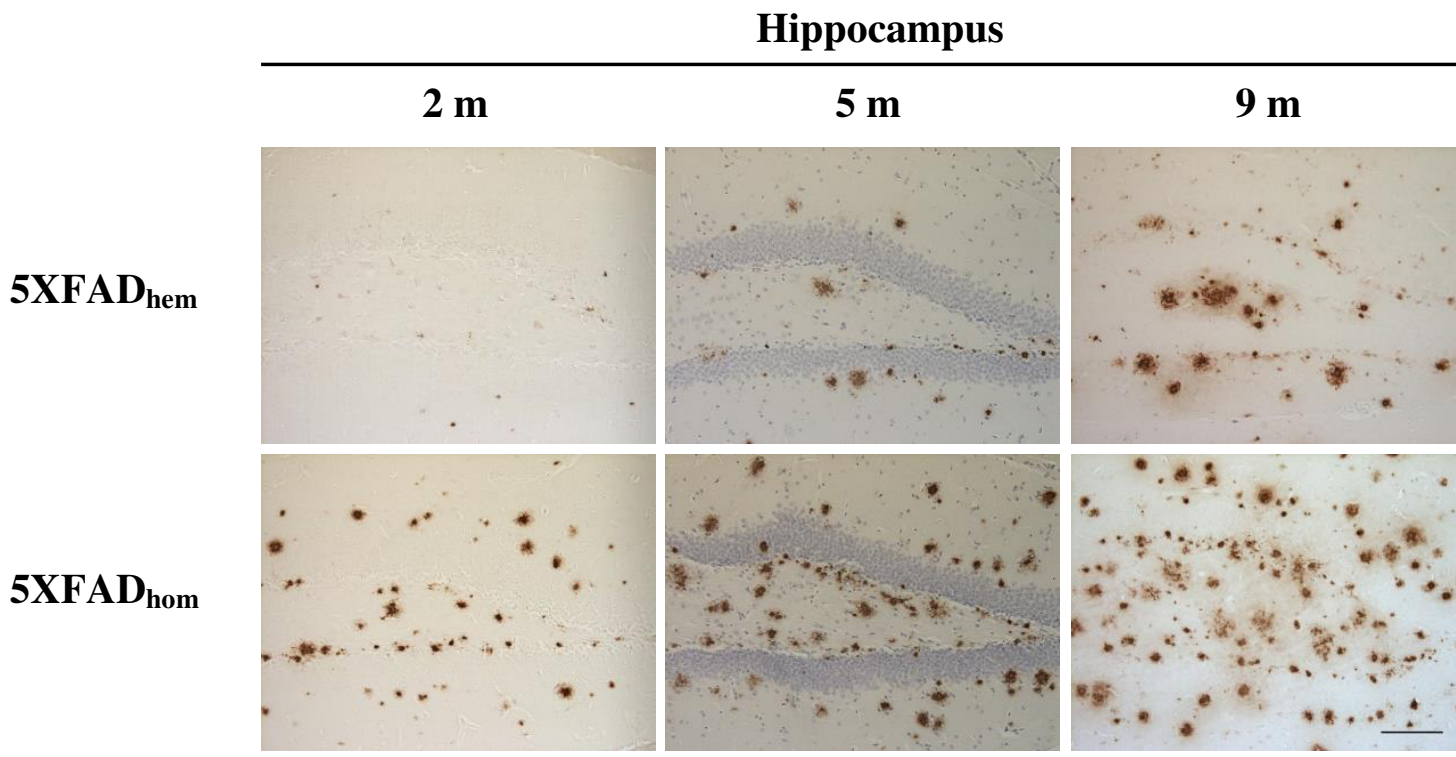

Thalamus

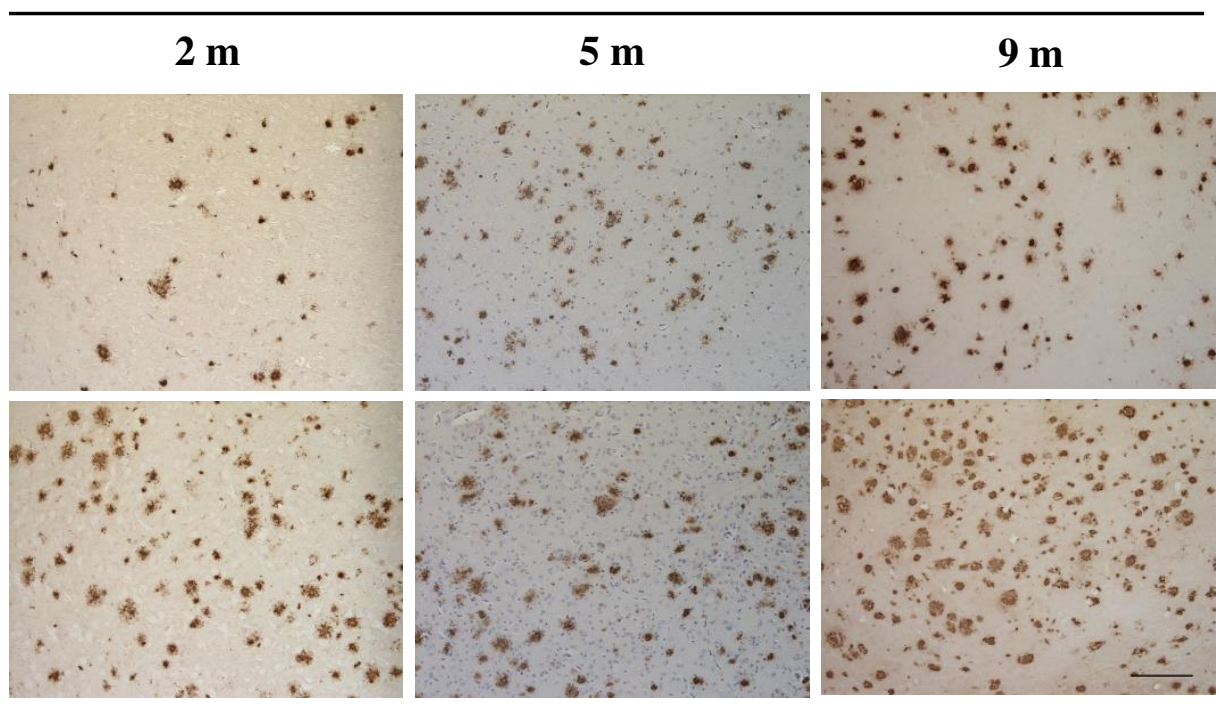

Subiculum

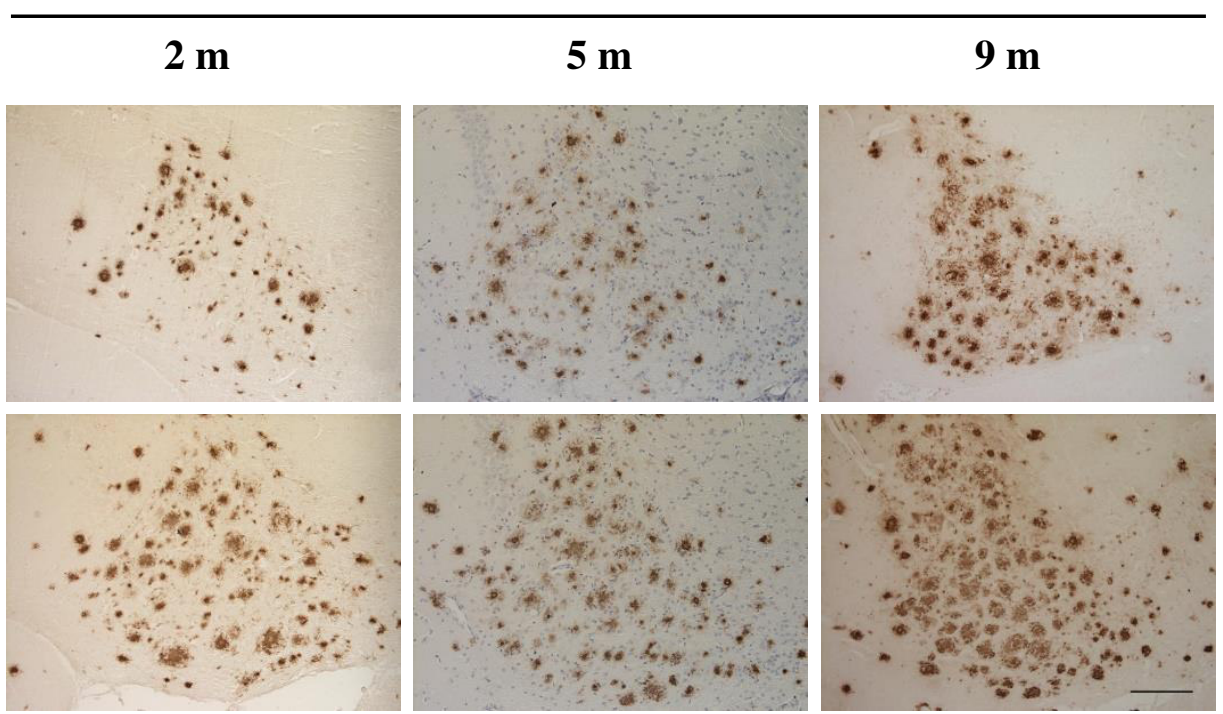

Figure 3. 5: Illustration of extracellular $A \beta$ plaque load in hippocampus, subiculum and thalamus. Strong increase of amyloid load in $5 \mathrm{XFAD}_{\text {hom }}$ mice in all analysed brain areas at all time points compared to $5 \mathrm{XFAD}_{\text {hem. }}$ Scale bar: $100 \mu \mathrm{m}$. 
Cortex
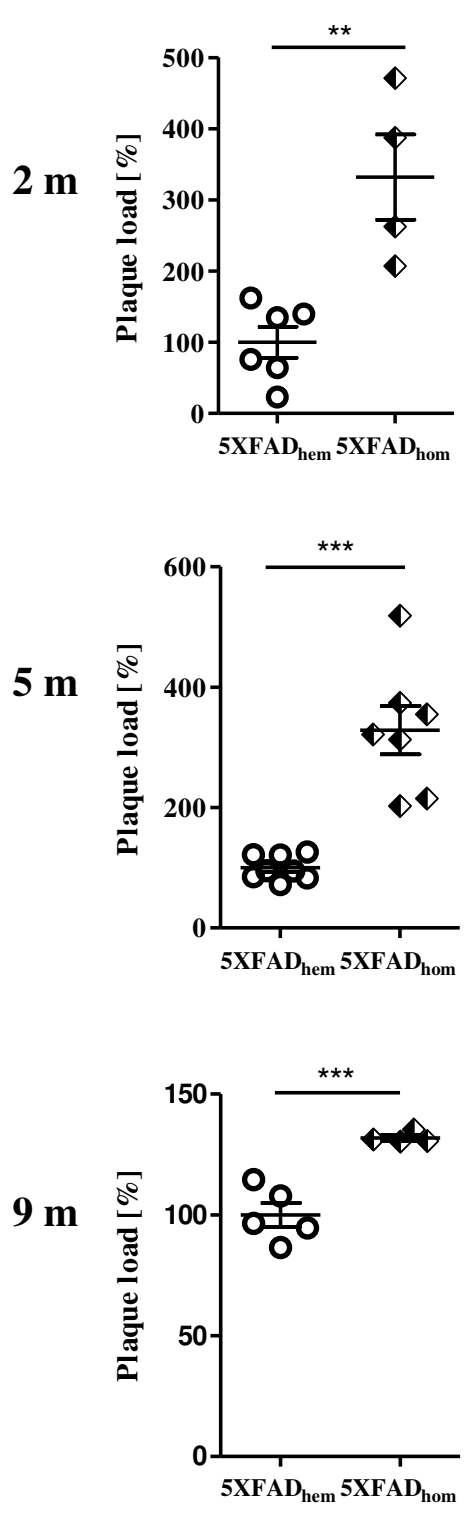

Hippocampus
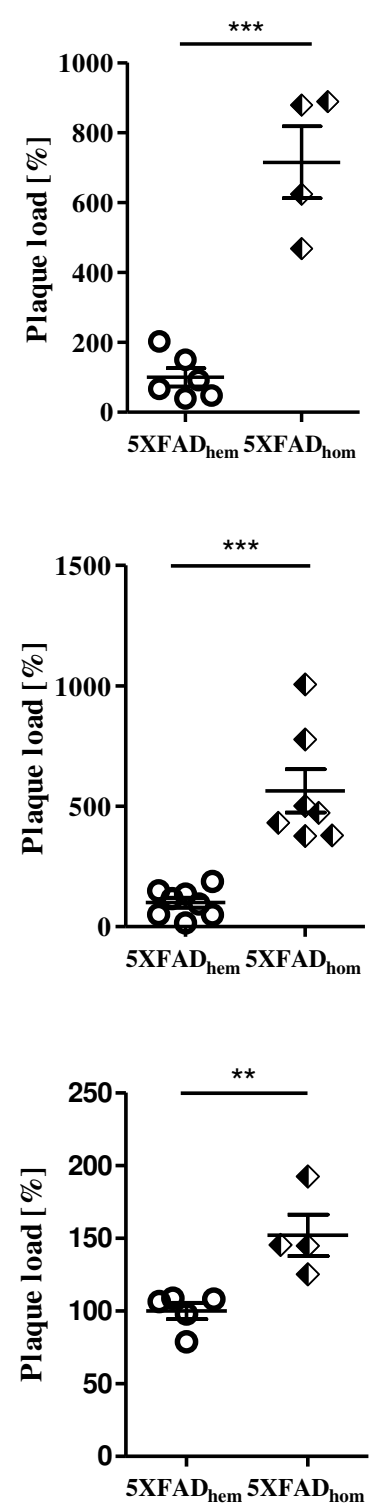

Subiculum
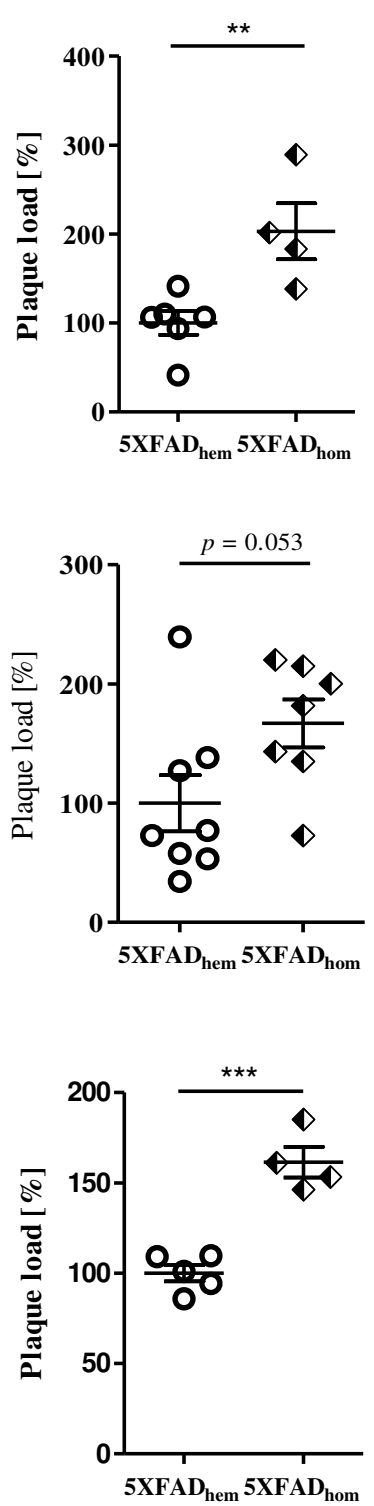
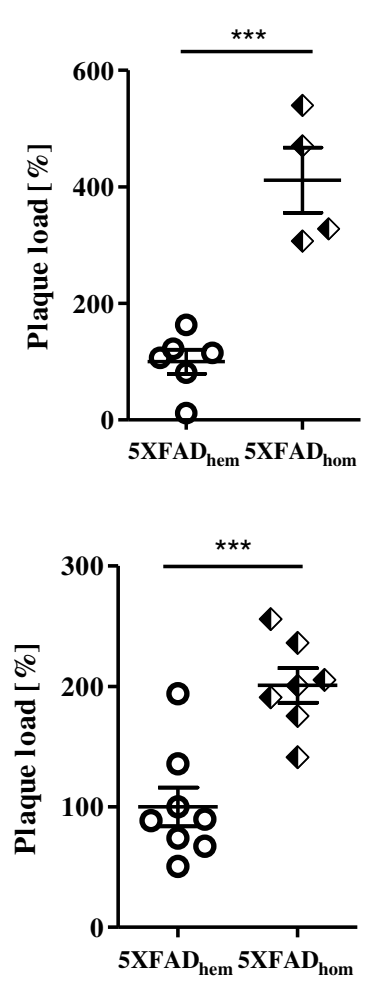

Thalamus

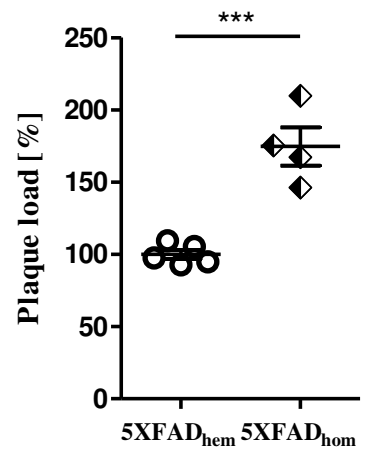

Figure 3. 6: Quantification of extracellular $A \beta$ plaque load in cortex, hippocampus, subiculum and thalamus. $5 \mathrm{XFAD}_{\text {hom }}$ mice showed a strongly increased plaque load in all analysed brain areas at all time points compared to age-matched hemizygous 5XFAD. For better comparison, the relative A $\beta$ load is expressed with $5 \mathrm{XFADhet}$ mice as the reference parameter. $\mathrm{m}=$ months. Data presented as mean \pm s.e.m; $* * * p<0.001, * * p$ $<0.01, * p<0.05$.

\subsubsection{Quantification of neurofilament-positive axonal spheroids}

The sagittal brain sections of $5 X_{F A D}$ hem and $5 X F A D_{\text {hom }}$ mice of 9 months of age were stained against neurofilament-L (a neurofilament subunit) in order to evaluate the gene dosage-dependent effect on the extent of axonal pathology. Neurofilament-positive axonal swellings (spheroids) that were not located in the immediate vicinity of amyloid plaques were counted in the pons and spinal cord. 

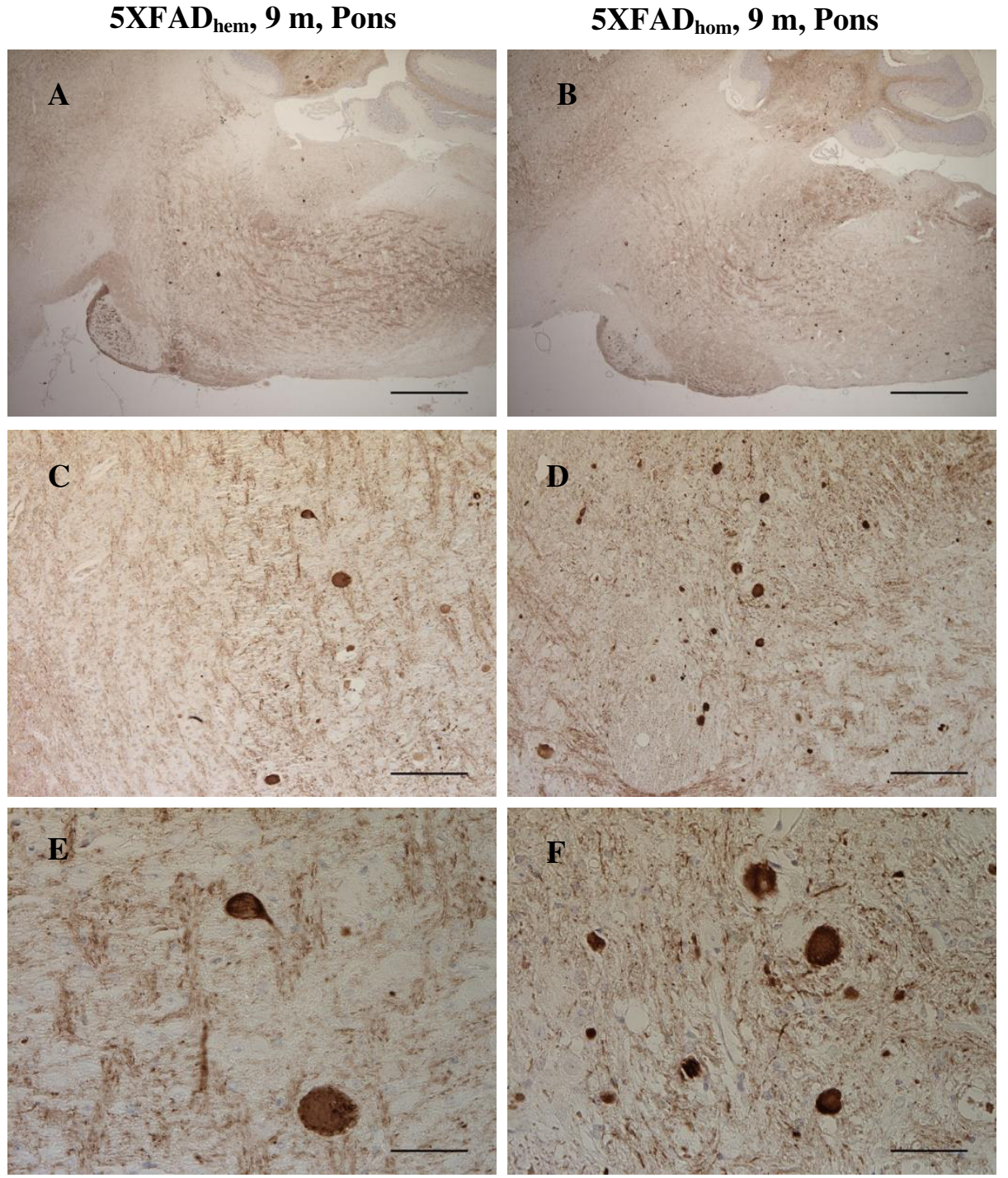

G

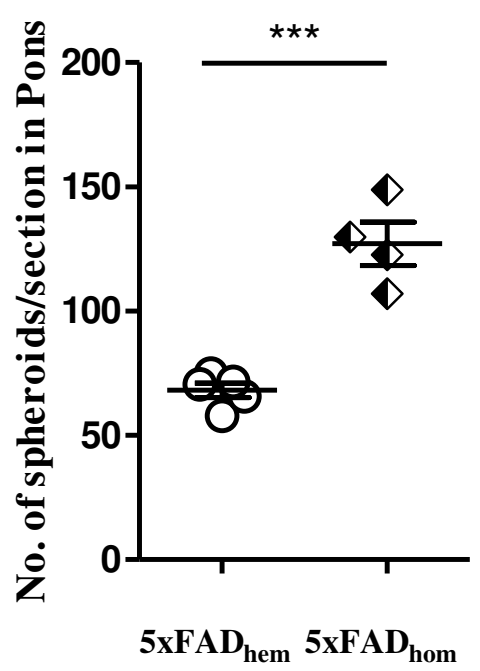

H

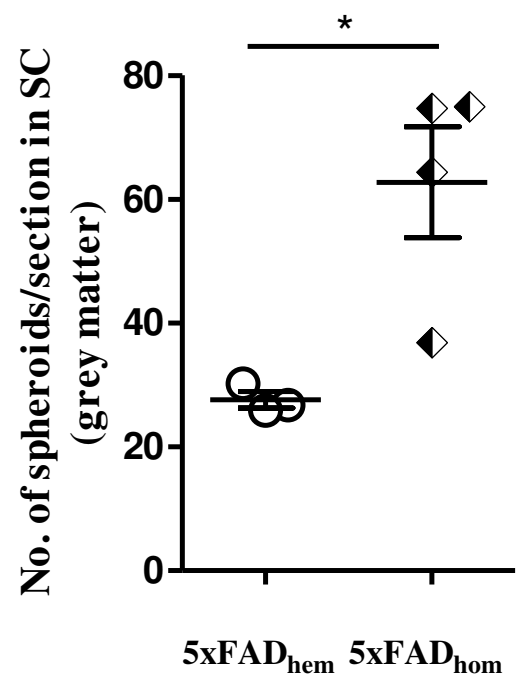

Figure 3. 7: Quantification of neurofilament-positive axonal spheroids in the pons and spinal cord. (A-F) Neurofilament-L stained brain sections; scale bar (A-B): $500 \mu \mathrm{m}$; scale bar (C-D): $100 \mu \mathrm{m}$; scale bar (E-F): $33 \mu \mathrm{m}$. (G-H) The 9-month-old $5 \mathrm{XFAD}_{\text {hom }}$ mice revealed a significantly increased number of axonal swellings in both regions compared to $5 \mathrm{XFAD}_{\text {hem }}$ mice. $\mathrm{m}=$ months. Data presented as mean \pm s.e.m; $* * * p<$ $0.001, * p<0.05$. 
The number of spheroids per section in the pons in $5 \mathrm{XFAD}_{\text {hom }}$ mice was significantly increased $(127.1 \pm 17.35 ; n=4)$ compared to age-matched $5 \mathrm{XFAD}_{\text {hem }}$ mice $(67.11 \pm 6.27 ; \mathrm{n}=$ $5 ; \mathrm{p}=0.0002)$. The same was valid for the gray matter of the spinal cord. The number of axonal swellings in $5 \mathrm{XFAD}_{\text {hom }}$ was also strongly increased $(62.74 \pm 17.93 ; \mathrm{n}=4)$ compared to age-matched $5 X_{F A D}$ hem mice $(27.64 \pm 2.27 ; n=3)$ (Figure 3.7).

The results of the Project II have been published in Richard et al. 2015. 


\section{DISCUSSION}

Mouse models are very important tools in the $\mathrm{AD}$ research that help us to understand the pathogenesis of $\mathrm{AD}$, prevent it or develop treatments against it. In the present study two mouse models of AD have been investigated. A novel model Tg4-42, which is a model for sporadic $\mathrm{AD}$, and a well-established 5XFAD model for familial $\mathrm{AD}$ were the subjects of this work.

The first project aimed to quantify the neuron loss and neurogenesis in the DG of 6-month-old Tg4-42 hom mice and to determine the effects of environmental enrichment, in particular physical activity, on these histopathological features. In the second project, the 5XFAD model was investigated in terms of amyloidosis and axonal impairments under the influence of the gene dosage elevation.

In the following part of this work I would like to discuss the results of the present study.

\subsection{Project I: Physical activity ameliorates neuron loss and increases neurogen- esis in 6-month-old Tg4-42 ${ }_{\text {hom }}$ mice.}

Tg4-42 is a novel model for sporadic AD created by our group, which expresses exclusively $N$-truncated $A \beta_{4-42}$. $A \beta_{4-42}$ belongs to the major $A \beta$ isoforms in the $A D$ brain and its expression induces an age-dependent CA1 neuron loss associated with a severe memory decline (Bouter et al. 2013a).

As already mentioned, the aim of this study was to further characterize the $\operatorname{Tg} 4-42$ mouse model, investigate neuron loss in further hippocampal region such as dentate gyrus, and find out, whether physical, social and sensorial stimulation can prevent neuron loss and rescue neurogenesis in the dentate gyrus in Tg4-42 model. At the same time other colleagues in our group investigated, whether enriched living conditions can counteract CA1 neuron loss and ameliorate behavioural deficits in Tg4-42 mice (Hüttenrauch et al. 2016). The EE paradigm was used for both studies. The EE paradigm consists of a combination of voluntary exercise and cognitive stimulation. In the present study, homozygous $\mathrm{Tg} 4-42$ mice were housed under EE conditions. The EE housing was started before disease onset and continued until 6 months of age (the time point were SH mice display severe behavioural deficits and serious loss of CA1 hippocampal neurons). 
In this study, unbiased design-based stereology revealed that EE ameliorates neuron loss in homozygous Tg4-42 mice. The number of DG cells in 6-month-old Tg4-42 hom mice was restored to the levels of the wild type mice. In Hüttenrauch et al. (2016), our group also reported that robust deficits in spatial reference memory, impaired non-spatial learning and memory were rescued by EE housing conditions.

There are plenty of studies showing that increased cognitive activity and leisure-time physical activity during the life are associated with decreased risk of dementia and AD in old age (Rovio et al. 2005). It has been shown that enriched living conditions and especially physical activity have beneficial effect on cognitive performance (Kempermann et al. 1997; van Praag et al. 1999). With regard to this, EE paradigm was intensely investigated in terms of rescuing memory and learning abilities in animal models of neurodegenerative diseases (Nithianantharajah and Hannan 2006). Some results from human (Hamer and Chida 2009; Rolland et al. 2008) and animal studies (Görtz et al. 2008; Nichol et al. 2007) showed that physical activity and cognitive stimulation can prevent or slow down the AD progression. Many other reports on enriched environment also showed improvement in cognitive tests (Arendash et al. 2004; Costa et al. 2007; Jankowsky et al. 2005).

However, to the best of my current knowledge, no study was conducted, which shows the positive effects of environmental enrichment on the prevention of neuronal loss in AD mouse models so far. Our group previously published an EE study on APP/PS1KI mice, demonstrating that the housing condition had no influence on the selective loss of hippocampal CA1 neurons (Casas et al. 2004; Cotel et al. 2012). In contrast, the present study indeed shows a beneficial effect of physical activity on DG neuronal loss in an AD mouse model. An explanation for these at first glance conflicting results might be that the APP/PS1KI mouse model is a very robust and aggressive model of familial AD with several mutations, the effects of which cannot be ameliorated by rather mild interventions such as increased physical activity, cognitive and social stimulation. On the contrary, the Tg4-42 mouse model only expresses $A \beta_{4-42}$ and does not possess any mutations, and therefore rather represent a model of sporadic AD which might be able to be modified through environmental interventions like physical activity.

Some other AD mouse models upon enriched environment (TgCRND8, APP/PS1 $\triangle \mathrm{E} 9$ ) show a decreased amyloid plaque load (Adlard et al. 2005; Lazarov et al. 2005). Tg4-42 mice do not develop any extracellular amyloid plaques. They only secrete $A \beta_{4-42}$ soluble neurotoxic aggregates, predominantly in the hippocampus (Bouter et al. 2013a). It would be legitimate to assume that $\mathrm{Tg} 4-42$ mice would also display at least decreased levels of $\mathrm{A} \beta_{4-42}$. Strikingly, improved cognitive performance and reduced neuron loss in Tg4-42 $2_{\text {het }}$ mice housed under EE was not accompanied with decreased levels of $A \beta_{4-42}$ (Hüttenrauch et al. 2016). Hence, it can be hypothesized that the cognitive improvement and the neuroprotective effect induced by physical activity in Tg4-42 mice is not dependent to a simple reduction of $A \beta_{4-42}$ levels. Similar effects were observed in other mice models e.g. Tg2576 or APP23 demonstrating improved cognitive performance despite unchanged A $\beta$ levels (Arendash et al. 2004) or amyloid plaque load (Wolf et al. 2006). Therefore, in this case, it can be speculated that the beneficial effects of environmental interventions can be attributed to the expansion of cognitive reserve that helps coping with pathological changes in brain during the AD (Akbaraly et al. 2009; Albert et al. 1995). 
Interestingly, the quantitative analysis of the volume of the DG revealed no significant difference between three groups. The same was also observed in the APP/PS1KI mice, where the volume of DG also remained constant despite the loss of DG cells (Cotel et al. 2012). Microscopically, the DG of SH Tg4-42 hom mice showed loosened structure without shrinkage in comparison to the DG of EE Tg4-42 hom mice.

As already stated, neuron loss and cortical atrophy are very important neuropathological hallmarks in AD patients. However, not all transgenic models of AD do show substantial neuron loss. Neuronal loss has not been reported for some APP transgenic models such as PDAPP (Games et al. 1995), Tg2576 (Hsiao et al. 1996), and APP/PS1 $\triangle E 9$ (Borchelt et al. 1997). Mainly mouse models with multiple APP and PSEN-1 mutations display this cardinal feature of AD. For instance, 5XFAD mice show significant neuron loss in the fifth cortical layer of the frontal cortex from the 9 month of age. Similar to 5XFAD mice, substantial neuron loss is observed in the frontal cortex and CA1 region of hippocampus in APP/PS1K1 mice (Breyhan et al. 2009; Christensen et al. 2008; Eimer and Vassar 2013; Jawhar et al. 2012).

Interestingly, despite almost ubiquitous appearance of plaques in these mouse models, neuron loss occurs only in the regions with intraneuronal $A \beta$ accumulation. This observation speaks more likely against the toxic role of extracellular plaques and supports the theory about toxicity of intracellular $A \beta$ accumulation. In Tg4-42 mice, the dramatic progressive neuron loss also occurs in the regions of intracellular $A \beta_{4-42}$ accumulation. In the Tg442 mice, long-term exposure to $\mathrm{N}$-truncated $A \beta_{4-42}$ induces an age- and dose-dependent neuron loss in CA1, which obviously contributes to the learning and memory deficits (Bouter et al. 2013a). These findings support the theory that intraneuronal $A \beta$, in particular $\mathrm{A} \beta_{4-42}$, plays a crucial role in the process of neurodegeneration. The fact that Tg4-42 mice do not develop extracellular amyloid plaques but still demonstrate the majority of the cardinal AD features also speaks in favour of intracellular amyloid hypothesis (Bouter et al. 2013a).

While studying the impact of environmental enrichment, in particular physical activity, one of the main issues is the neurogenic effect of the EE paradigm. Increased hippocampal neurogenesis is one of the major effects that have been observed in the adult brains of rodents (Kempermann et al. 1997; Kempermann et al. 1998a; Kempermann et al. 1998b; van Praag et al. 1999). In this study, the analysis of doublecortin-positive new-born neurons in the DG of the standard housed Tg4- $42_{\text {hom }}$ mice revealed a very low amount of doublecortin-positive cells with atrophic, in the DCX-staining not visible dendrites in comparison with SH wild-types. On the contrary, the EE-housed Tg4-42 $2_{\text {hom }}$ mice showed strongly increased number of DCX-positive cells comparing to age-matched standard housed Tg4$42_{\text {hom }}$ mice. Housing under EE conditions was able to restore the number of new-born neurons almost to the levels of SH wild-types. Qualitative analysis of the dendrites of DCXpositive cells on the basis of microscopy revealed also a stronger dendritic branching in EE-housed Tg4-42 $2_{\text {hom }}$ mice comparing to the standard-housed Tg4-42 ${ }_{\text {hom }}$ group. Nevertheless, SH Tg4-42 $2_{\text {hom }}$ mice hadless pronounced dendrites (shorter and less branched) in comparison to the wild-type aged-matched SH-mice. These findings are in line with with previous reports claiming that running stimulates the dendritic arbor of newborn cells in the 
DG (Dostes et al. 2016; Marlatt et al. 2013). The explanation for restored total numbers of DG-cells in EE Tg4-42 hom mice could be the rescued neurogenesis that leads to replacement of dead cells. Decreased hippocampal neurogenesis has been previously described also in other mouse models of AD such as PDAPP (Donovan et al. 2006), TgCRND8 (Herring et al. 2009), and 3xTg mice (Rodríguez et al. 2008).

In conclusion, the present study is the first to demonstrate that long-term physical activity has a preventive effect on $A \beta$-induced neuron loss and impaired neurogenesis in a transgenic mouse model of sporadic AD. Our results support the data obtained in retrospective epidemiological studies on human AD. Translating the findings of this animal study to the human AD patients, it can be assumed that physical activity and enriched environment may be beneficial in patients with mild cognitive impairment (before obvious disease onset). However, there are still a lot of questions remained unanswered. What is the reason of impaired neurogenesis in the SGZ of the DG? Is it due to the direct influence of $\mathrm{A} \beta_{4-42}$ or due to the possible inflammatory climate (Verret et al. 2007)? Some studies showed a strong negative correlation between the number of surviving new born neurons and the number of activated microglia cells (Ekdahl et al. 2003) and described new-born neurons as highly vulnerable to inflammation. Does the neurogenic dysfunction originate from impaired cell cycle or from impaired differentiation or migration of new-born cells (Zitnik and Martin 2002)? Are physical activity and cognitive stimulation reliable enough for rescuing dying neurons? Further investigations need to be done also in order to clarify whether a later exposure to physical activity and environmental enrichment, i.e. after the onset of cognitive and behavioural deficits, would reduce the gravity of these symptoms and would still ameliorate neuron loss and neurogenesis.

Unfortunately for $\mathrm{AD}$ research, rodents do not develop AD spontaneously during the aging process (Sarasa and Pesini 2009). The transgenic mouse models of AD based on mutations in AD-related genes are relevant, in the first line, for the investigation of familial AD, which makes up barely $1 \%$ of AD cases (Zetterberg and Mattsson 2014). The majority of cases are caused by sporadic AD. Regarding to this fact, the translation of findings in animal studies to the situation of sporadic AD patients is complicated. Thereby, the $\mathrm{Tg} 4-42$ mouse model, which reflects the sporadic form of the disease, is an important tool in the investigation of sporadic AD.

\subsection{Project II: Gene dosage-dependent increase of amyloid pathology and ax- onal degeneration in 5XFAD ${ }_{\text {hom }}$ mice}

The 5XFAD mouse model is a double transgenic A PPP/PS1 mouse line co-expressing five familial $\mathrm{AD}$ mutations that are inherited together. This leads to massive and early plaque formation, intraneuronal $A \beta$ aggregation, elevated $A \beta_{42}$ levels, behavioural deficits and neuron loss in the cortical layer V. 5XFAD was previously described as one of the few 
AD models with several cardinal features of AD including neuron loss (Jawhar et al. 2012; Eimer and Vassar 2013; Oakley et al. 2006).

The present study aimed at performing an analysis of a potential gene dosagedependent effect on neuropathological features in 5XFAD mice such as plaque load and axonopathy. In order to elevate the transgene expression and the $A \beta$ levels, a homozygous 5XFAD line was created (Richard et al. 2015). Breeding transgenic mouse models of AD to homozygosity is an established method to aggravate the extent and accelerate the onset of pathological alterations. Increasing the gene dosage has been previously performed in other mouse models of AD such as PDAPP (German et al. 2003), ARTE10 (Willuweit et al. 2009) and Tg4-42 (Bouter et al. 2013a).

In this study, it has been shown that the levels of extracellularly deposited amyloid are significantly elevated in the $5 \mathrm{XFAD}$ hom groups compared to the respective $5 \mathrm{XFAD}_{\text {hem }}$ groups in all analysed brain regions at all time points. In particular significant increase in A $\beta$ plaque load was found in the cortex, the hippocampus, the subiculum and the thalamus. This was most evident at the earlier stages (2-month-old and 5-month-old mice) with 3- to 7-fold increases. Interestingly, 9-month-old mice showed only moderate increase in A $\beta$ plaque load. This might reflect a saturation effect. This finding is consistent with the initial description of 5XFAD, which although not quantified, indicated a slower accumulation of A $\beta$ between 6 and 9 months of age (Oakley et al. 2006). A similar plateau stage has also been observed in the APP/PS1KI and in the PDAPP model (DeMattos et al. 2012; Wirths et al. 2010c).

In the present study, besides the aggravation of the amyloid plaque pathology, it was demonstrated that $5 \mathrm{XFAD}_{\text {hom }}$ mice at 9 months of age show severe axonal degeneration in the pons and the spinal cord compared to age-matched heterozygous mice. The immunohistochemistry on brain and spinal cord with anti-neurofilament-L antibody revealed a significantly increased number of axonal swellings per section in $5 \mathrm{XFAD}_{\text {hom }}$ compared to $5 \mathrm{XFAD}_{\text {hem }}$ mice. This correlates positively with the earlier onset of motor deficits (Richard et al. 2015). An age-dependent axonopathy in brain and spinal cord of 5XFAD has been previously described (Jawhar et al. 2012). Spinal cord pathology including formation of dystrophic neurites, amyloid plaques, and motor neuron degeneration has been also described in several other mouse models overexpressing mutant APP (Christensen et al. 2014; Seo et al. 2010; Wirths et al. 2006; Wirths et al. 2007; Yuan et al. 2013). Furthermore, intracellular $A \beta$ accumulation in spinal cord motor neurons has been observed in several APP transgenic e.g. APP/PS1 (Wirths et al. 2006) or APP/PS1KI (Christensen et al. 2014; Wirths et al. 2007), as well as in mice expressing only A $\beta$ peptides without APP overexpression such as APP48 mice expressing A $\beta_{1-42}$ (Abramowski et al. 2012) or TBA42 mice expressing $A \beta_{\mathrm{pE} 3-42}$ (Wittnam et al. 2012). Several studies suggested that fibrillary $A \beta$ leads to the formation of axonal swellings (Grace et al. 2002; Selkoe 2002). This subsequently causes local accumulation of cell components such as vesicles, mitochondria, vacuoles, that leads to the starvation of synapses and dying-back neurons.

Axonal damage analogous to axonopathy in 5XFAD mice has been also reported in AD patients. For example, signs of axonopathy and decreased axonal transport rate have 
been shown in vivo by $\mathrm{MRI}^{5}$ (Minoshima and Cross 2008) as well as in post mortem studies (Dai et al. 2002; Xiao et al. 2011). Whereas there is a plethora of literature on cognitive decline during the course of $\mathrm{AD}$, detailed reports on motor impairments in $\mathrm{AD}$ are limited (Albers et al. 2015; Wirths and Bayer 2008). Impaired motor performance has been reported in later disease stages (Albers et al. 2015; Cummings 1990), but there is an evidence that they are also present in mildly demented AD patients (Goldman et al. 1999). Some studies suggested that motor deficits even might be a characteristic feature of early AD pathology (Scarmeas et al. 2004) and bear some predictive value (Scarmeas et al. 2005).

\section{Other gene dosage-dependent effects in the 5XFAD model}

In Richard et al. (2015), along with the described acceleration of amyloid plaque deposition and significantly increased axonal degeneration, we have shown that the development of behavioural impairment was also accelerated and the levels of both TBS- and SDS-soluble $\mathrm{A} \beta$ peptides were significantly elevated in the $5 \mathrm{XFAD}_{\text {hom }}$ groups compared to the respective $5 \mathrm{XFAD}_{\text {hem }}$ groups. Behavioural experiments, phenotypical characterisation of the mice and quantification of TBS- and SDS-soluble A $\beta$ peptides were performed by my colleague Bernhard Richard.

The onset of behaviour deficits was considerably shifted towards earlier time points in the $5 X{ }^{2} D_{\text {hom }}$ strain. For example, a robust significant impairment of the spatial reference memory was observed already at the age of 5 months for the $5 \mathrm{XFAD}_{\text {hom }}$ group, whereas age-matched $5 \mathrm{XFAD}_{\text {hem }}$ did not show any impairment in corresponding tasks. Due to homozygosity, not only the onset of behavioural impairment was shifted toward earlier time points. It was accompanied by an aggravation of other alterations such as reduced body weight and faster development of a motor phenotype for $5 \mathrm{XFAD}_{\mathrm{hom}}$ (Richard et al. 2015).

For the quantification of soluble A $\beta$ peptides whole-brain lysates (TBS and SDSfraction) of 2- and 5-month-old animals were subjected to quantitative ELISA measurement. ELISA measurements were performed by Sandra Baches, Dept. of Neuropathology, Heinrich Heine Universität Düsseldorf, Germany. Water-soluble $A \beta_{1-42}$ levels, as well as SDS-soluble $A \beta_{1-40}$ and $A \beta_{1-42}$ were significantly elevated in $5 X_{F A D}$ hom animals ( 2 and 5 months of age) compared to age-matched $5 X_{F A D}$ hem (Richard et al. 2015). These findings are consistent with the increased plaque load observed in $5 \mathrm{XFAD}_{\text {hom }}$ mice.

It cannot be discriminated whether extracellular amyloid depositions or soluble $A \beta$ are responsible for behavioural deficits. There is evidence that amyloid plaques do have neurotoxic effects, especially if $A \beta$ depositions adopt a fibrillary $\beta$-pleated conformation (Urbanc et al. 2002). However, it has been also shown that mice without amyloid plaque accumulation but considerable levels of soluble $A \beta$ peptides demonstrate an altered synaptic structure (Price et al. 2014).

In summary, homozygous 5XFAD mice demonstrate abundant $\mathrm{A} \beta$ pathology, a robust behavioural phenotype with deficits in reference learning and memory, and pronounced

\footnotetext{
${ }^{5}$ Magnetic Resonance Imaging
} 
axonopathy. Increasing the transgene dose in 5XFAD leads to increased transgene expression and elevated $A \beta$ levels that subsequently accelerates the onset of related behavioural and histopathological alterations, and aggravates them. The early onset of these deficits makes $5 \mathrm{XFAD}_{\text {hom }}$ a well-suited model for preclinical studies within a short time frame. Moreover, due to the strongly increased levels of $A \beta$, the model might especially facilitate the analysis of intracellular $A \beta$ pathology at very early time points. 


\section{SUMMARY}

The present study aimed at further investigation of two transgenic mouse models of Alzheimer's disease (AD): Tg4-42 and 5XFAD.

The transgenic mouse model Tg4-42 was recently developed in our group and displays the variety of typical $\mathrm{AD}$ features. It does not possess any mutations but overexpresses $\mathrm{N}$ terminally truncated $A \beta_{4-42}$. Thereby, it is likely that $\mathrm{Tg} 4-42$ represents the model for sporadic form of $\mathrm{AD}$. The Tg4-42 model is unique because of its massive neuron loss and severe cognitive deficits induced through intraneuronal accumulation of N-terminally truncated $\mathrm{A} \beta_{4-42}$ without amyloid plaque formation.

So long as current drug treatments have only modest effects on the symptomatic course of $\mathrm{AD}$, environmental interventions are of great interest in order to prevent $\mathrm{AD}$ or delay its onset. In the first project of my study it was shown that environmental enrichment, combining physical activity and cognitive enhancement, leads to an amelioration of neuronal loss in Tg4-42. Furthermore, the evidences were provided that physical activity triggers neurogenesis in Tg4-42 and rescues it to the wild-type levels.

For this study, the group of Tg4-42 (EE) starting at age of 2 months. At the age of 5 months, mice were tested for hippocampus-dependent memory, motor abilities, and anxiety levels (projects of my colleagues). Their cerebral tissue was subsequently analysed for neurogenesis and neuronal loss in the dentate gyrus (DG) (present study). Therefore, group of Tg4-42 hom mice housed under EE conditions was compared to the age-matched $\mathrm{Tg} 4-42_{\text {hom }}$ group and wild-type group from the standard housing ( $\mathrm{SH}$ ). Unbiased design-based stereology revealed that EE ameliorates neuron loss $\mathrm{Tg} 4-42_{\mathrm{hom}}$ mice. The number of DG cells in 6-month-old $\mathrm{Tg} 4-42_{\mathrm{hom}}$ mice was restored to the levels of the wild-type mice. In order to quantify the neurogenesis, immunohistochemistry with doublecortin (DCX) has been performed. DCX is a microtubuleassociated protein expressed in all migrating neuronal precursors in central nervous system. Using stereology, DCX-positive new-born neurons were counted in the DG. The analysis of the standard housed $\mathrm{Tg} 4-42_{\text {hom }}$ mice revealed a very low amount of DCXpositive cells with impaired dendritic arborization in comparison with $\mathrm{SH}$ wild-types. Quite the contrary to SH-housed Tg4- $42_{\text {hom }}$ mice, the EE-housed mice showed strongly increased number of DCX-positive cells with much more pronounced processes. EE was able to restore the number of new-born neurons in $\mathrm{Tg} 4-42_{\text {hom }}$ mice almost to the levels of SH wild-types.

The results of this study should be considered together with the results from other colleagues from our research group providing evidences that enhanced physical activity improves motor abilities and counteracts declining memory performance. 
In summary, the results obtained here might indicate the importance of environmental enrichment, in particular increased physical activity, as a potential strategy in the prevention of dementia. They also validate Tg4-42 mouse model as a well-suitable model for investigation of the sporadic form of $\mathrm{AD}$.

The focus of the second project laid on the 5XFAD mouse model. The 5XFAD is a widely used model, which is based on the expression of mutant amyloid precursor protein and presenilin-1 genes, and represents thereby a mouse model for familial AD. It is a typical model for early plaque formation, intraneuronal $A \beta$ aggregation, neuron loss and robust behavioural deficits.

This project aimed at investigation the gene dosage dependence of the neuropathological features in 5XFAD. Therefore a homozygous 5XFAD line was created to elevate transgene expression and $A \beta$ production. In order to evaluate the effect of increased gene dosage on the amyloid pathology and axonopathy, hemizygous and homozygous 5XFAD mice were compared. Sagittal brain sections of hemizygous and homozygous 5XFAD mice at 2, 5 and 9 months of age were stained with an $A \beta[N]$ antibody, which detects an $\mathrm{N}$ terminal $A \beta$ epitope, and the amyloid plaque load was quantified in the cortex, hippocampus (hippocampal region CA and DG), retrohippocampal region (subiculum)) and thalamus. Furthermore, dystrophic neurites were counted in the pons and spinal cord in order to evaluate the extent of axonopathy. In the present study, it was shown that the levels of extracellular amyloid formations are significantly elevated in the $5 \mathrm{XFAD}_{\text {hom }}$ groups compared to the respective $5 \mathrm{XFAD}_{\text {hem }}$ groups in all analysed brain regions at all time points. 3to 7-fold increases $A \beta$ plaque load were found at the 2- and 5-month-stages. 9-month-old mice showed a lesser increase in $A \beta$ plaque load that might reflect a saturation effect. Along with the aggravation of the amyloidosis, it was demonstrated that 9-month-old $5 \mathrm{XFAD}$ hom mice show severe axonal degeneration in the pons and the spinal cord compared to age-matched heterozygous mice.

The results of this study should be considered together with the results from my colleagues, especially from Bernhard Richard, who performed a phenotypical characterisation of $5 \mathrm{XFAD}_{\text {hom }}$ mice and investigated a behavioural phenotype with deficits in reference learning and memory: besides the described acceleration of amyloid plaque pathology and strongly increased axonal degeneration, it was shown that the development of memory deficits and behavioural impairment was also accelerated.

Thereby, homozygous 5XFAD mice represent a model with several advantages comparing with the heterozygous mice. Namely, $5 \mathrm{XFAD}_{\text {hom }}$ mice develop amyloid pathology much more rapidly as well as a neurological phenotype. Bred to homozygosity 5XFAD mice show a strongly reduced age of onset of behavioural symptoms and neuropathological alterations. These advantages can speed up preclinical studies. In conclusion, the results obtained in this study have broadened the knowledge about the 5XFAD mouse model and further validated this model as an appropriate model for investigation of $\mathrm{AD}$ and for analysis of $\mathrm{A} \beta$ species, memory and behaviour. 


\section{REFERENCES}

Abramowski D, Rabe S, Upadhaya AR, Reichwald J, Danner S, Staab D, Capetillo-Zarate E, Yamaguchi H, Saido TC, Wiederhold K-H, et al. (2012): Transgenic Expression of Intraneuronal $A \beta 42$ But Not $A \beta 40$ Leads to Cellular $A \beta$ Lesions, Degeneration, and Functional Impairment without Typical Alzheimer's Disease Pathology. J Neurosci 32, 1273-1283

Adalbert R, Nogradi A, Babetto E, Janeckova L, Walker SA, Kerschensteiner M, Misgeld T, Coleman MP (2009): Severely dystrophic axons at amyloid plaques remain continuous and connected to viable cell bodies. Brain J Neurol 132, 402-416

Adlard PA, Perreau VM, Pop V, Cotman CW (2005): Voluntary Exercise Decreases Amyloid Load in a Transgenic Model of Alzheimer's Disease. J Neurosci 25, 4217-4221

Akbaraly TN, Portet F, Fustinoni S, Dartigues J-F, Artero S, Rouaud O, Touchon J, Ritchie K, Berr C (2009): Leisure activities and the risk of dementia in the elderly: results from the ThreeCity Study. Neurology $\underline{73}, 854-861$

Albers MW, Gilmore GC, Kaye J, Murphy C, Wingfield A, Bennett DA, Boxer AL, Buchman AS, Cruickshanks KJ, Devanand DP, et al. (2015): At the interface of sensory and motor dysfunctions and Alzheimer's disease. Alzheimers Dement 11, 70-98

Albert MS, Jones K, Savage CR, Berkman L, Seeman T, Blazer D, Rowe JW (1995): Predictors of cognitive change in older persons: MacArthur studies of successful aging. Psychol Aging $\underline{10}, 578-589$

Albert MS, DeKosky ST, Dickson D, Dubois B, Feldman HH, Fox NC, Gamst A, Holtzman DM, Jagust WJ, Petersen RC, et al. (2011): The diagnosis of mild cognitive impairment due to Alzheimer's disease: Recommendations from the National Institute on Aging-Alzheimer's Association workgroups on diagnostic guidelines for Alzheimer's disease. Alzheimers Dement J Alzheimers Assoc 7, 270-279

Aldwin CM, Gilmer DF: Health, illness, and optimal aging biological and psychosocial perspectives. 2nd ed.; Springer, New York 2013

Alexandru A, Jagla W, Graubner S, Becker A, Bäuscher C, Kohlmann S, Sedlmeier R, Raber KA, Cynis H, Rönicke R, et al. (2011): Selective Hippocampal Neurodegeneration in Transgenic Mice Expressing Small Amounts of Truncated $A \beta$ Is Induced by Pyroglutamate-A $\beta$ Formation. J Neurosci $\underline{31}, 12790-12801$

Alladi S, Bak TH, Duggirala V, Surampudi B, Shailaja M, Shukla AK, Chaudhuri JR, Kaul S (2013): Bilingualism delays age at onset of dementia, independent of education and immigration status. Neurology $\underline{81}, 1938-1944$

Allan LM, Ballard CG, Rowan EN, Kenny RA (2009): Incidence and Prediction of Falls in Dementia: A Prospective Study in Older People. PLoS ONE $\underline{4}$, e5521 
Almeida CG, Takahashi RH, Gouras GK (2006): $\beta$-Amyloid Accumulation Impairs Multivesicular Body Sorting by Inhibiting the Ubiquitin-Proteasome System. J Neurosci 26, 4277-4288

Alonso AC, Grundke-Iqbal I, Iqbal K (1996): Alzheimer's disease hyperphosphorylated tau sequesters normal tau into tangles of filaments and disassembles microtubules. Nat Med $\underline{2}$, $783-787$

Alzheimer A (1907): Über eine eigenartige Erkrankung der Hirnrinde. Allg Z Für Psychiatr PsychGerichtl Med 64, 146-148

Alzheimer A (1911): Über eigenartige Krankheitsfälle des späteren Alters. Z Für Gesammte Neurol Psychiatr $\underline{4}, 356-385$

Alzheimer's Association (2012): 2012 Alzheimer's disease facts and figures. Alzheimers Dement J Alzheimers Assoc $\underline{8}, 131-168$

American Psychiatric Association: Diagnostic and statistical manual of mental disorders, fourth edition: primary care version (DSM-IV-PC). American Psychiatric Association, Washington, DC 1995

Anderson RN, DeTurk PB (2002): United States life tables, 1999. Natl Vital Stat Rep Cent Dis Control Prev Natl Cent Health Stat Natl Vital Stat Syst $\underline{50}$, 1-39

Annaert W, Strooper BD (2002): A Cell Biological Perspective on Alzheimer's Disease. Annu Rev Cell Dev Biol 18, 25-51

Aprahamian I, Martinelli JE, Neri AL, Yassuda MS (2010): The accuracy of the Clock Drawing Test compared to that of standard screening tests for Alzheimer's disease: results from a study of Brazilian elderly with heterogeneous educational backgrounds. Int Psychogeriatr $\underline{22}, 64-71$

Arenaza-Urquijo EM, Wirth M, Chételat G (2015): Cognitive reserve and lifestyle: moving towards preclinical Alzheimer's disease. Front Aging Neurosci 134

Arendash GW, Garcia MF, Costa DA, Cracchiolo JR, Wefes IM, Potter H (2004): Environmental enrichment improves cognition in aged Alzheimer's transgenic mice despite stable betaamyloid deposition. Neuroreport $\underline{15}, 1751-1754$

Arnold SE, Hyman BT, Flory J, Damasio AR, Hoesen GWV (1991): The Topographical and Neuroanatomical Distribution of Neurofibrillary Tangles and Neuritic Plaques in the Cerebral Cortex of Patients with Alzheimer's Disease. Cereb Cortex 1, 103-116

Arriagada PV, Growdon JH, Hedley-Whyte ET, Hyman BT (1992): Neurofibrillary tangles but not senile plaques parallel duration and severity of Alzheimer's disease. Neurology $\underline{42}, 631-$ 639

Aytan N, Choi J-K, Carreras I, Kowall NW, Jenkins BG, Dedeoglu A (2013): Combination therapy in a transgenic model of Alzheimer's disease. Exp Neurol 250, 228-238

Ballard C, Gauthier S, Corbett A, Brayne C, Aarsland D, Jones E (2011): Alzheimer's disease. The Lancet $\underline{377}, 1019-1031$

Barbas CF, Burton DR, Scott JK, Silverman GJ (2007): Quantitation of DNA and RNA. Cold Spring Harb Protoc 2007, pdb.ip47 
Bard F, Cannon C, Barbour R, Burke R-L, Games D, Grajeda H, Guido T, Hu K, Huang J, Johnson-Wood K, et al. (2000): Peripherally administered antibodies against amyloid $\beta$-peptide enter the central nervous system and reduce pathology in a mouse model of Alzheimer disease. Nat Med $\underline{6}, 916-919$

Bard F, Barbour R, Cannon C, Carretto R, Fox M, Games D, Guido T, Hoenow K, Hu K, JohnsonWood K, et al. (2003): Epitope and isotype specificities of antibodies to $\beta$-amyloid peptide for protection against Alzheimer's disease-like neuropathology. Proc Natl Acad Sci U S A $\underline{100}, 2023-2028$

Bartus RT, Dean RL, Beer B, Lippa AS (1982): The cholinergic hypothesis of geriatric memory dysfunction. Science 217, 408-414

Baumeister R (1999): The physiological role of presenilins in cellular differentiation: lessons from model organisms. Eur Arch Psychiatry Clin Neurosci 249, 280-287

Bayer TA, Wirths O (2008): Review on the APP/PS1KI mouse model: intraneuronal A $\beta$ accumulation triggers axonopathy, neuron loss and working memory impairment. Genes Brain Behav $\underline{7}, 6-11$

Bayer TA, Wirths O (2010): Intracellular Accumulation of Amyloid-Beta - A Predictor for Synaptic Dysfunction and Neuron Loss in Alzheimer's Disease. Front Aging Neurosci $\underline{2}$

Bayer TA, Wirths O (2014): Focusing the amyloid cascade hypothesis on N-truncated Abeta peptides as drug targets against Alzheimer's disease. Acta Neuropathol (Berl) 127, 787-801

Beauquis J, Pavía P, Pomilio C, Vinuesa A, Podlutskaya N, Galvan V, Saravia F (2013): Environmental enrichment prevents astroglial pathological changes in the hippocampus of APP transgenic mice, model of Alzheimer's disease. Exp Neurol 239, 28-37

Benilova I, Karran E, De Strooper B (2012): The toxic A $\beta$ oligomer and Alzheimer's disease: an emperor in need of clothes. Nat Neurosci $\underline{15}, 349-357$

Benzing WC, Wujek JR, Ward EK, Shaffer D, Ashe KH, Younkin SG, Brunden KR (1999): Evidence for glial-mediated inflammation in aged APPSW transgenic mice. Neurobiol Aging $\underline{20}, 581-589$

Bertram L, Lill CM, Tanzi RE (2010): The Genetics of Alzheimer Disease: Back to the Future. Neuron $\underline{68}, 270-281$

Bhattacharya S, Haertel C, Maelicke A, Montag D (2014): Galantamine Slows Down Plaque Formation and Behavioral Decline in the 5XFAD Mouse Model of Alzheimer's Disease. PLoS ONE $\underline{9}$, e89454

Bialystok E, Craik FIM, Freedman M (2007): Bilingualism as a protection against the onset of symptoms of dementia. Neuropsychologia $\underline{45}, 459-464$

Bibl M, Gallus M, Welge V, Esselmann H, Wolf S, Rüther E, Wiltfang J (2012): Cerebrospinal fluid amyloid- $\beta$ 2-42 is decreased in Alzheimer's, but not in frontotemporal dementia. J Neural Transm $\underline{119}, 805-813$

Bickeböller H, Campion D, Brice A, Amouyel P, Hannequin D, Didierjean O, Penet C, Martin C, Pérez-Tur J, Michon A, et al. (1997): Apolipoprotein E and Alzheimer disease: genotypespecific risks by age and sex. Am J Hum Genet 60, 439-446 
Bien J, Jefferson T, Causević M, Jumpertz T, Munter L, Multhaup G, Weggen S, Becker-Pauly C, Pietrzik CU (2012): The metalloprotease meprin $\beta$ generates amino terminal-truncated amyloid $\beta$ peptide species. J Biol Chem $\underline{287}, 33304-33313$

Billings LM, Oddo S, Green KN, McGaugh JL, LaFerla FM (2005): Intraneuronal A $\beta$ Causes the Onset of Early Alzheimer's Disease-Related Cognitive Deficits in Transgenic Mice. Neuron $\underline{45}, 675-688$

Blackmore DG, Golmohammadi MG, Large B, Waters MJ, Rietze RL (2009): Exercise Increases Neural Stem Cell Number in a Growth Hormone-Dependent Manner, Augmenting the Regenerative Response in Aged Mice. STEM CELLS 27, 2044-2052

Blázquez G, Cañete T, Tobeña A, Giménez-Llort L, Fernández-Teruel A (2014): Cognitive and emotional profiles of aged Alzheimer's disease $(3 \times \mathrm{TgAD})$ mice: Effects of environmental enrichment and sexual dimorphism. Behav Brain Res $\underline{268}$, 185-201

Blennow K, de Leon MJ, Zetterberg H (2006): Alzheimer's disease. Lancet 368, 387-403

Boldrini M, Underwood MD, Hen R, Rosoklija GB, Dwork AJ, John Mann J, Arango V (2009): Antidepressants increase neural progenitor cells in the human hippocampus. Neuropsychopharmacology $\underline{34}, 2376-2389$

Borchelt DR, Ratovitski T, van Lare J, Lee MK, Gonzales V, Jenkins NA, Copeland NG, Price DL, Sisodia SS (1997): Accelerated Amyloid Deposition in the Brains of Transgenic Mice Coexpressing Mutant Presenilin 1 and Amyloid Precursor Proteins. Neuron 19, 939-945

Bouter Y: The role of amyloid beta 4-42 in the etiology of Alzheimer's disease. Dr.rer.nat. Diss. Göttingen 2014

Bouter Y, Dietrich K, Wittnam JL, Rezaei-Ghaleh N, Pillot T, Papot-Couturier S, Lefebvre T, Sprenger F, Wirths O, Zweckstetter M, Bayer TA (2013a): N-truncated amyloid $\beta$ (A $\beta$ ) 442 forms stable aggregates and induces acute and long-lasting behavioral deficits. Acta Neuropathol (Berl) 126, 189-205

Bouter Y, Dietrich K, Wittnam J, Pillot T, Papot-Couturier S, Lefebvre T, Sprenger F, Wirths O, Bayer T (2013b): Tg4-42: A new mouse model of Alzheimer's disease-N-truncated amyloid $\beta(\mathrm{A} \beta)$ 4-42 induces severe neuron loss and behavioral deficits. Alzheimers Dement $\underline{9}$, P498-P499

Bouter Y, Kacprowski T, Weissmann R, Dietrich K, Borgers H, Brauß A, Sperling C, Wirths O, Albrecht M, Jensen LR, et al. (2014): Deciphering the molecular profile of plaques, memory decline and neuron loss in two mouse models for Alzheimer's disease by deep sequencing. Front Aging Neurosci $\underline{6}, 75$

Braak H, Braak E (1991): Neuropathological stageing of Alzheimer-related changes. Acta Neuropathol (Berl) $\underline{82}$, 239-259

Breyhan H, Wirths O, Duan K, Marcello A, Rettig J, Bayer TA (2009): APP/PS1KI bigenic mice develop early synaptic deficits and hippocampus atrophy. Acta Neuropathol (Berl) 117, $677-685$

Brun A, Englund E (1981): Regional pattern of degeneration in Alzheimer's disease: neuronal loss and histopathological grading. Histopathology $\underline{5}, 549-564$

Bullock R, Dengiz A (2005): Cognitive performance in patients with Alzheimer's disease receiving cholinesterase inhibitors for up to 5 years. Int J Clin Pract 59, 817-822 
Bullock R, Touchon J, Bergman H, Gambina G, He Y, Rapatz G, Nagel J, Lane R (2005): Rivastigmine and donepezil treatment in moderate to moderately-severe Alzheimer's disease over a 2-year period. Curr Med Res Opin 21, 1317-1327

Cameron HA, Gould E (1994): Adult neurogenesis is regulated by adrenal steroids in the dentate gyrus. Neuroscience $\underline{61}, 203-209$

Carrillo-Mora P, Luna R, Colín-Barenque L (2014): Amyloid beta: multiple mechanisms of toxicity and only some protective effects? Oxid Med Cell Longev 2014, 795375

Casas C, Sergeant N, Itier J-M, Blanchard V, Wirths O, van der Kolk N, Vingtdeux V, van de Steeg E, Ret G, Canton T, et al. (2004): Massive CA1/2 Neuronal Loss with Intraneuronal and N-Terminal Truncated A $\beta 42$ Accumulation in a Novel Alzheimer Transgenic Model. Am J Pathol 165, 1289-1300

Castellani RJ, Zhu X, Lee H-G, Smith MA, Perry G (2009): Molecular Pathogenesis of Alzheimer's Disease: Reductionist versus Expansionist Approaches. Int J Mol Sci 10, 13861406

Castellano JM, Kim J, Stewart FR, Jiang H, DeMattos RB, Patterson BW, Fagan AM, Morris JC, Mawuenyega KG, Cruchaga C, et al. (2011): Human apoE Isoforms Differentially Regulate Brain Amyloid- $\beta$ Peptide Clearance. Sci Transl Med $\underline{3}$, 89ra57

Cavanaugh SE, Pippin JJ, Barnard ND (2014): Animal models of Alzheimer disease: historical pitfalls and a path forward. ALTEX 31, 279-302

Cayre M, Canoll P, Goldman JE (2009): Cell migration in the normal and pathological postnatal mammalian brain. Prog Neurobiol $\underline{88}, 41-63$

Chen G, Chen KS, Knox J, Inglis J, Bernard A, Martin SJ, Justice A, McConlogue L, Games D, Freedman SB, Morris RGM (2000): A learning deficit related to age and $\beta$-amyloid plaques in a mouse model of Alzheimer's disease. Nature 408, 975-979

Chen Y, Tian Z, Liang Z, Sun S, Dai C, Lee MH, LaFerla FM, Grundke-Iqbal I, Iqbal K, Liu F, Gong C-X (2012): Brain Gene Expression of a Sporadic (icv-STZ Mouse) and a Familial Mouse Model (3xTg-AD Mouse) of Alzheimer's Disease. PLoS ONE $\underline{7}$, e51432

Chen Y, Liang Z, Blanchard J, Dai C-L, Sun S, Lee MH, Grundke-Iqbal I, Iqbal K, Liu F, Gong CX (2013): A non-transgenic mouse model (icv-STZ mouse) of Alzheimer's disease: similarities to and differences from the transgenic model (3xTg-AD mouse). Mol Neurobiol $\underline{47}$, $711-725$

Chevalier-Larsen E, Holzbaur ELF (2006): Axonal transport and neurodegenerative disease. Biochim Biophys Acta BBA - Mol Basis Dis 1762, 1094-1108

Chevallier NL, Soriano S, Kang DE, Masliah E, Hu G, Koo EH (2005): Perturbed Neurogenesis in the Adult Hippocampus Associated with Presenilin-1 A246E Mutation. Am J Pathol 167, $151-159$

Chishti MA, Yang D-S, Janus C, Phinney AL, Horne P, Pearson J, Strome R, Zuker N, Loukides J, French J, et al. (2001): Early-onset Amyloid Deposition and Cognitive Deficits in Transgenic Mice Expressing a Double Mutant Form of Amyloid Precursor Protein 695. J Biol Chem 276, 21562-21570

Cho W-H, Park J-C, Kim D-H, Kim M-S, Lee S-Y, Park H, Kang J-H, Yeon S-W, Han J-S (2014): ID1201, the ethanolic extract of the fruit of Melia toosendan ameliorates impairments in 
spatial learning and reduces levels of amyloid beta in 5XFAD mice. Neurosci Lett $\underline{583}$, $170-175$

Chow VW, Mattson MP, Wong PC, Gleichmann M (2010): An overview of APP processing enzymes and products. Neuromolecular Med $\underline{12}, 1-12$

Christensen DZ, Kraus SL, Flohr A, Cotel M-C, Wirths O, Bayer TA (2008): Transient intraneuronal $\mathrm{A} \beta$ rather than extracellular plaque pathology correlates with neuron loss in the frontal cortex of APP/PS1KI mice. Acta Neuropathol (Berl) 116, 647-655

Christensen DZ, Bayer TA, Wirths O (2010): Intracellular A $\beta$ triggers neuron loss in the cholinergic system of the APP/PS1KI mouse model of Alzheimer's disease. Neurobiol Aging $\underline{31}$, 1153-1163

Christensen DZ, Huettenrauch M, Mitkovski M, Pradier L, Wirths O (2014): Axonal degeneration in an Alzheimer mouse model is PS1 gene dose dependent and linked to intraneuronal A $\beta$ accumulation. Front Aging Neurosci $\underline{6}, 139$

Citron M, Teplow DB, Selkoe DJ (1995): Generation of amyloid beta protein from its precursor is sequence specific. Neuron 14, 661-670

Coleman M (2005): Axon degeneration mechanisms: commonality amid diversity. Nat Rev Neurosci $\underline{6}, 889-898$

Corder EH, Saunders AM, Strittmatter WJ, Schmechel DE, Gaskell PC, Small GW, Roses AD, Haines JL, Pericak-Vance MA (1993): Gene dose of apolipoprotein E type 4 allele and the risk of Alzheimer's disease in late onset families. Science 261, 921-923

Corder EH, Saunders AM, Risch NJ, Strittmatter WJ, Schmechel DE, Gaskell PC, Rimmler JB, Locke PA, Conneally PM, Schmader KE, et al. (1994): Protective effect of apolipoprotein E type 2 allele for late onset Alzheimer disease. Nat Genet 7, 180-184

Costa DA, Cracchiolo JR, Bachstetter AD, Hughes TF, Bales KR, Paul SM, Mervis RF, Arendash GW, Potter H (2007): Enrichment improves cognition in AD mice by amyloid-related and unrelated mechanisms. Neurobiol Aging $\underline{28}, 831-844$

Cotel M-C: The extent of Alzheimer pathology in mice: an attempt toward disease modification by environmental intervention. Ph.D. Diss. Göttingen 2009

Cotel M-C, Jawhar S, Christensen DZ, Bayer TA, Wirths O (2012): Environmental enrichment fails to rescue working memory deficits, neuron loss, and neurogenesis in APP/PS1KI mice. Neurobiol Aging $\underline{33}$, 96-107

Couillard-Despres S, Winner B, Schaubeck S, Aigner R, Vroemen M, Weidner N, Bogdahn U, Winkler J, Kuhn H-G, Aigner L (2005): Doublecortin expression levels in adult brain reflect neurogenesis. Eur J Neurosci 21, 1-14

Cras P, Smith MA, Richey PL, Siedlak SL, Mulvihill P, Perry G (1995): Extracellular neurofibrillary tangles reflect neuronal loss and provide further evidence of extensive protein crosslinking in Alzheimer disease. Acta Neuropathol (Berl) $\underline{89}$, 291-295

Crews FT, Nixon K (2003): Alcohol, neural stem cells, and adult neurogenesis. Alcohol Res Health J Natl Inst Alcohol Abuse Alcohol 27, 197-204 
Crowe M, Andel R, Pedersen NL, Johansson B, Gatz M (2003): Does participation in leisure activities lead to reduced risk of Alzheimer's disease? A prospective study of Swedish twins. J Gerontol B Psychol Sci Soc Sci 58, 249-255

Cruz-Orive L-M (1985): Estimating Volumes from Systematic Hyperplane Sections. J Appl Probab $\underline{22}, 518-530$

Cummings JL: Clinical diagnosis of Alzheimer's disease. In: Cummings JL, Miller BL (eds.), Alzheimer's disease: Treatment and long-term management; Marcel Dekker, New York 1990, 3-19

Cummings JL, Vinters HV, Cole GM, Khachaturian ZS (1998): Alzheimer's disease: etiologies, pathophysiology, cognitive reserve, and treatment opportunities. Neurology $\underline{51}, 2-17$; discussion 65-67

Dai J, Buijs RM, Kamphorst W, Swaab DF (2002): Impaired axonal transport of cortical neurons in Alzheimer's disease is associated with neuropathological changes. Brain Res 948, 138-144

Dash PK, Mach SA, Moore AN (2001): Enhanced neurogenesis in the rodent hippocampus following traumatic brain injury. J Neurosci Res $\underline{63}, 313-319$

Dawson GR, Seabrook GR, Zheng H, Smith DW, Graham S, O’Dowd G, Bowery BJ, Boyce S, Trumbauer ME, Chen HY, et al. (1999): Age-related cognitive deficits, impaired long-term potentiation and reduction in synaptic marker density in mice lacking the $\beta$-amyloid precursor protein. Neuroscience $\underline{90}, 1-13$

DeKosky ST, Scheff SW (1990): Synapse loss in frontal cortex biopsies in Alzheimer's disease: correlation with cognitive severity. Ann Neurol 27, 457-464

Delaère P, Duyckaerts C, He Y, Piette F, Hauw JJ (1991): Subtypes and differential laminar distributions of beta A4 deposits in Alzheimer's disease: relationship with the intellectual status of 26 cases. Acta Neuropathol (Berl) $\underline{81}, 328-335$

DeMattos RB, Bales KR, Cummins DJ, Dodart J-C, Paul SM, Holtzman DM (2001): Peripheral anti-A $\beta$ antibody alters CNS and plasma $A \beta$ clearance and decreases brain $A \beta$ burden in a mouse model of Alzheimer's disease. Proc Natl Acad Sci U S A $\underline{98}, 8850-8855$

DeMattos RB, Lu J, Tang Y, Racke MM, DeLong CA, Tzaferis JA, Hole JT, Forster BM, McDonnell PC, Liu F, et al. (2012): A Plaque-Specific Antibody Clears Existing $\beta$-amyloid Plaques in Alzheimer's Disease Mice. Neuron 76, 908-920

De-Paula VJ, Radanovic M, Diniz BS, Forlenza OV (2012): Alzheimer's disease. Subcell Biochem $\underline{65}, 329-352$

De Strooper B, Vassar R, Golde T (2010): The secretases: enzymes with therapeutic potential in Alzheimer disease. Nat Rev Neurol $\underline{6}, 99-107$

Devi L, Ohno M (2010): Genetic reductions of $\beta$-site amyloid precursor protein-cleaving enzyme 1 and amyloid- $\beta$ ameliorate impairment of conditioned taste aversion memory in 5XFAD Alzheimer's disease model mice. Eur J Neurosci 31, 110-118

Devi L, Ohno M (2013): Mechanisms that lessen benefits of $\beta$-secretase reduction in a mouse model of Alzheimer's disease. Transl Psychiatry $\underline{3}$, e284

De Vos KJ, Grierson AJ, Ackerley S, Miller CCJ (2008): Role of axonal transport in neurodegenerative diseases. Annu Rev Neurosci $\underline{31}, 151-173$ 
Dickson DW, Crystal HA, Mattiace LA, Masur DM, Blau AD, Davies P, Yen S-H, Aronson MK (1992): Identification of normal and pathological aging in prospectively studied nondemented elderly humans. Neurobiol Aging $\underline{13}, 179-189$

Ditaranto K, Tekirian TL, Yang AJ (2001): Lysosomal Membrane Damage in Soluble A $\beta$ Mediated Cell Death in Alzheimer's Disease. Neurobiol Dis $\underline{8}, 19-31$

Dodart J-C, Mathis C, Saura J, Bales KR, Paul SM, Ungerer A (2000): Neuroanatomical Abnormalities in Behaviorally Characterized APPV717F Transgenic Mice. Neurobiol Dis $\underline{7}, 71-$ 85

Dodart J-C, Bales KR, Gannon KS, Greene SJ, DeMattos RB, Mathis C, DeLong CA, Wu S, Wu X, Holtzman DM, Paul SM (2002): Immunization reverses memory deficits without reducing brain $\mathrm{A} \beta$ burden in Alzheimer's disease model. Nat Neurosci $\underline{5}, 452-457$

Dong J, Zhou M, Wu X, Du M, Wang X (2012): Memantine combined with environmental enrichment improves spatial memory and alleviates Alzheimer's disease-like pathology in senescence-accelerated prone-8 (SAMP8) mice. J Biomed Res 26, 439-447

Donovan MH, Yazdani U, Norris RD, Games D, German DC, Eisch AJ (2006): Decreased adult hippocampal neurogenesis in the PDAPP mouse model of Alzheimer's disease. J Comp Neurol $\underline{495}, 70-83$

Dostes S, Dubreucq S, Ladevèze E, Marsicano G, Abrous DN, Chaouloff F, Koehl M (2016): Running per se stimulates the dendritic arbor of newborn dentate granule cells in mouse hippocampus in a duration-dependent manner. Hippocampus $\underline{26}, 282-288$

Drechsel DN, Hyman AA, Cobb MH, Kirschner MW (1992): Modulation of the dynamic instability of tubulin assembly by the microtubule-associated protein tau. Mol Biol Cell $\underline{3}, 1141-$ 1154

Dudal S, Krzywkowski P, Paquette J, Morissette C, Lacombe D, Tremblay P, Gervais F (2004): Inflammation occurs early during the $A \beta$ deposition process in TgCRND8 mice. Neurobiol Aging 25, 861-871

Duyckaerts C, Potier M-C, Delatour B (2008): Alzheimer disease models and human neuropathology: similarities and differences. Acta Neuropathol (Berl) $\underline{115}, 5-38$

Duyckaerts C, Delatour B, Potier M-C (2009): Classification and basic pathology of Alzheimer disease. Acta Neuropathol (Berl) 118, 5-36

Eckman CB, Mehta ND, Crook R, Perez-tur J, Prihar G, Pfeiffer E, Graff-Radford N, Hinder P, Yager D, Zenk B, et al. (1997): A new pathogenic mutation in the APP gene (I716V) increases the relative proportion of A beta 42(43). Hum Mol Genet $\underline{6}$, 2087-2089

Eimer WA, Vassar R (2013): Neuron loss in the 5XFAD mouse model of Alzheimer's disease correlates with intraneuronal $\mathrm{A} \beta 42$ accumulation and Caspase-3 activation. Mol Neurodegener $\underline{8}, 2$

Ekdahl CT, Claasen J-H, Bonde S, Kokaia Z, Lindvall O (2003): Inflammation is detrimental for neurogenesis in adult brain. Proc Natl Acad Sci 100, 13632-13637

Elder GA, Gama Sosa MA, De Gasperi R (2010): Transgenic Mouse Models of Alzheimer's Disease. Mt Sinai J Med J Transl Pers Med 77, 69-81 
Esch FS, Keim PS, Beattie EC, Blacher RW, Culwell AR, Oltersdorf T, McClure D, Ward PJ (1990): Cleavage of amyloid beta peptide during constitutive processing of its precursor. Science $\underline{248}, 1122-1124$

Esh C, Patton L, Kalback W, Kokjohn TA, Lopez J, Brune D, Newell AJ, Beach T, Schenk D, Games D, et al. (2005): Altered APP Processing in PDAPP (Val717 $\rightarrow$ Phe) Transgenic Mice Yields Extended-Length A $\beta$ Peptides. Biochemistry 44, 13807-13819

Feart C, Samieri C, Barberger-Gateau P (2015): Mediterranean diet and cognitive health: an update of available knowledge. Curr Opin Clin Nutr Metab Care $\underline{18}, 51-62$

Fernández-Vizarra P, Fernández AP, Castro-Blanco S, Serrano J, Bentura ML, Martínez-Murillo R, Martínez A, Rodrigo J (2004): Intra- and extracellular Abeta and PHF in clinically evaluated cases of Alzheimer's disease. Histol Histopathol $\underline{19}, 823-844$

Ferrer I, Boada Rovira M, Sánchez Guerra ML, Rey MJ, Costa-Jussá F (2004): Neuropathology and pathogenesis of encephalitis following amyloid-beta immunization in Alzheimer's disease. Brain Pathol Zurich Switz 14, 11-20

Ferreri F, Pauri F, Pasqualetti P, Fini R, Dal Forno G, Rossini PM (2003): Motor cortex excitability in Alzheimer's disease: A transcranial magnetic stimulation study. Ann Neurol $\underline{53}, 102$ 108

Ferri A, Sanes JR, Coleman MP, Cunningham JM, Kato AC (2003): Inhibiting Axon Degeneration and Synapse Loss Attenuates Apoptosis and Disease Progression in a Mouse Model of Motoneuron Disease. Curr Biol 13, 669-673

Fiandaca MS, Mapstone ME, Cheema AK, Federoff HJ (2014): The critical need for defining preclinical biomarkers in Alzheimer's disease. Alzheimers Dement 10, 196-212

Fiol-deRoque MA, Gutierrez-Lanza R, Terés S, Torres M, Barceló P, Rial RV, Verkhratsky A, Escribá PV, Busquets X, Rodríguez JJ (2013): Cognitive recovery and restoration of cell proliferation in the dentate gyrus in the 5XFAD transgenic mice model of Alzheimer's disease following 2-hydroxy-DHA treatment. Biogerontology 14, 763-775

Fitchett AE, Collins SA, Barnard CJ, Cassaday HJ (2005): Subordinate male mice show longlasting differences in spatial learning that persist when housed alone. Neurobiol Learn Mem $\underline{84}, 247-251$

Flannery RB (2002): Treating learned helplessness in the elderly dementia patient: preliminary inquiry. Am J Alzheimers Dis Other Demen 17, 345-349

Folstein MF, Folstein SE, McHugh PR (1975): "Mini-mental state": A practical method for grading the cognitive state of patients for the clinician. J Psychiatr Res $\underline{12}$, 189-198

Foster PP (2015): Role of physical and mental training in brain network configuration. Front Aging Neurosci 7, 117

Fratiglioni L, Paillard-Borg S, Winblad B (2004): An active and socially integrated lifestyle in late life might protect against dementia. Lancet Neurol $\underline{3}, 343-353$

Frost JL, Liu B, Kleinschmidt M, Schilling S, Demuth H-U, Lemere CA (2012): Passive Immunization against Pyroglutamate-3 Amyloid- $\beta$ Reduces Plaque Burden in Alzheimer-Like Transgenic Mice: A Pilot Study. Neurodegener Dis 10, 265-270 
Furukawa K, Sopher BL, Rydel RE, Begley JG, Pham DG, Martin GM, Fox M, Mattson MP (1996): Increased Activity-Regulating and Neuroprotective Efficacy of $\alpha$-SecretaseDerived Secreted Amyloid Precursor Protein Conferred by a C-Terminal Heparin-Binding Domain. J Neurochem 67, 1882-1896

Galimberti D, Scarpini E (2012): Progress in Alzheimer's disease. J Neurol 259, 201-211

Games D, Adams D, Alessandrini R, Barbour R, Borthelette P, Blackwell C, Carr T, Clemens J, Donaldson T, Gillespie F, et al. (1995): Alzheimer-type neuropathology in transgenic mice overexpressing V717F $\beta$-amyloid precursor protein. Nature $\underline{373}$, 523-527

Games D, Buttini M, Kobayashi D, Schenk D, Seubert P (2006): Mice as models: transgenic approaches and Alzheimer's disease. J Alzheimers Dis JAD ㅁ, 133-149

German DC, Yazdani U, Speciale SG, Pasbakhsh P, Games D, Liang C-L (2003): Cholinergic neuropathology in a mouse model of Alzheimer's disease. J Comp Neurol 462, 371-381

Giannakopoulos P, Herrmann FR, Bussière T, Bouras C, Kövari E, Perl DP, Morrison JH, Gold G, Hof PR (2003): Tangle and neuron numbers, but not amyloid load, predict cognitive status in Alzheimer's disease. Neurology $\underline{60}, 1495-1500$

Glenner GG, Wong CW (1984): Alzheimer's disease: Initial report of the purification and characterization of a novel cerebrovascular amyloid protein. Biochem Biophys Res Commun 120, $885-890$

Goate A, Chartier-Harlin M-C, Mullan M, Brown J, Crawford F, Fidani L, Giuffra L, Haynes A, Irving N, James L, et al. (1991): Segregation of a missense mutation in the amyloid precursor protein gene with familial Alzheimer's disease. Nature $\underline{349}, 704-706$

Goedert M, Jakes R (2005): Mutations causing neurodegenerative tauopathies. Biochim Biophys Acta BBA - Mol Basis Dis 1739, 240-250

Goedert M, Spillantini MG (2006): A Century of Alzheimer's Disease. Science $\underline{314}$, 777-781

Gold BT (2015): Lifelong bilingualism and neural reserve against Alzheimer's disease: A review of findings and potential mechanisms. Behav Brain Res 281, 9-15

Goldman WP, Baty JD, Buckles VD, Sahrmann S, Morris JC (1999): Motor dysfunction in mildly demented AD individuals without extrapyramidal signs. Neurology $\underline{53}, 956-962$

Gómez-Isla T, Price JL, McKeel DW, Morris JC, Growdon JH, Hyman BT (1996): Profound loss of layer II entorhinal cortex neurons occurs in very mild Alzheimer's disease. J Neurosci Off J Soc Neurosci 16, 4491-4500

Gómez-Isla T, Hollister R, West H, Mui S, Growdon JH, Petersen RC, Parisi JE, Hyman BT (1997): Neuronal loss correlates with but exceeds neurofibrillary tangles in Alzheimer's disease. Ann Neurol 41, 17-24

Görtz N, Lewejohann L, Tomm M, Ambrée O, Keyvani K, Paulus W, Sachser N (2008): Effects of environmental enrichment on exploration, anxiety, and memory in female TgCRND8 Alzheimer mice. Behav Brain Res $\underline{191}$, 43-48

Götz J, Chen F, Dorpe J van, Nitsch RM (2001): Formation of Neurofibrillary Tangles in P301L Tau Transgenic Mice Induced by Aß42 Fibrils. Science 293, 1491-1495 
Gouras GK, Tsai J, Naslund J, Vincent B, Edgar M, Checler F, Greenfield JP, Haroutunian V, Buxbaum JD, Xu H, et al. (2000): Intraneuronal A $\beta 42$ Accumulation in Human Brain. Am J Pathol 156, 15-20

Grace EA, Rabiner CA, Busciglio J (2002): Characterization of neuronal dystrophy induced by fibrillar amyloid $\beta$ : implications for Alzheimer's disease. Neuroscience 114, 265-273

Grundke-Iqbal I, Iqbal K, Tung YC, Quinlan M, Wisniewski HM, Binder LI (1986): Abnormal phosphorylation of the microtubule-associated protein tau (tau) in Alzheimer cytoskeletal pathology. Proc Natl Acad Sci USA 푸, 4913-4917

Gu Y, Nieves JW, Stern Y, Luchsinger JA, Scarmeas N (2010): Food combination and alzheimer disease risk: A protective diet. Arch Neurol 67, 699-706

Gundersen HJ, Jensen EB (1987): The efficiency of systematic sampling in stereology and its prediction. J Microsc 147, 229-263

Gundersen HJG (1977): Notes on the estimation of the numerical density of arbitrary profiles: the edge effect. J Microsc 111, 219-223

Güntert A, Döbeli H, Bohrmann B (2006): High sensitivity analysis of amyloid-beta peptide composition in amyloid deposits from human and PS2APP mouse brain. Neuroscience $\underline{143}$, $461-475$

Guo J-P, Arai T, Miklossy J, McGeer PL (2006): A $\beta$ and tau form soluble complexes that may promote self aggregation of both into the insoluble forms observed in Alzheimer's disease. Proc Natl Acad Sci USA 103, 1953-1958

Gustke N, Trinczek B, Biernat J, Mandelkow EM, Mandelkow E (1994): Domains of tau protein and interactions with microtubules. Biochemistry 33 , 9511-9522

Gyure KA, Durham R, Stewart WF, Smialek JE, Troncoso JC (2001): Intraneuronal A $\beta$-Amyloid Precedes Development of Amyloid Plaques in Down Syndrome. Arch Pathol Lab Med $\underline{125}, 489-492$

Haass C (2004): Take five-BACE and the $\gamma$-secretase quartet conduct Alzheimer's amyloid $\beta$ peptide generation. EMBO J 23, 483-488

Haass C, Selkoe DJ (2007): Soluble protein oligomers in neurodegeneration: lessons from the Alzheimer's amyloid $\beta$-peptide. Nat Rev Mol Cell Biol $\underline{8}, 101-112$

Haass C, Lemere CA, Capell A, Citron M, Seubert P, Schenk D, Lannfelt L, Selkoe DJ (1995): The Swedish mutation causes early-onset Alzheimer's disease by beta-secretase cleavage within the secretory pathway. Nat Med $\underline{1}, 1291-1296$

Haass C, Kaether C, Thinakaran G, Sisodia S (2012): Trafficking and Proteolytic Processing of APP. Cold Spring Harb Perspect Med 2, a006270

Hafezparast M, Klocke R, Ruhrberg C, Marquardt A, Ahmad-Annuar A, Bowen S, Lalli G, Witherden AS, Hummerich H, Nicholson S, et al. (2003): Mutations in Dynein Link Motor Neuron Degeneration to Defects in Retrograde Transport. Science $\underline{300}$, 808-812

Hall CB, Lipton RB, Sliwinski M, Katz MJ, Derby CA, Verghese J (2009): Cognitive activities delay onset of memory decline in persons who develop dementia. Neurology $\underline{73}, 356-361$ 
Halliday GM, Double KL, Macdonald V, Kril JJ (2003): Identifying severely atrophic cortical subregions in Alzheimer's disease. Neurobiol Aging 24, 797-806

Hamer M, Chida Y (2009): Physical activity and risk of neurodegenerative disease: a systematic review of prospective evidence. Psychol Med $\underline{39}, 3-11$

Hardy J, Allsop D (1991): Amyloid deposition as the central event in the aetiology of Alzheimer's disease. Trends Pharmacol Sci $\underline{12}$, 383-388

Hardy JA, Higgins GA (1992): Alzheimer's disease: the amyloid cascade hypothesis. Science 256 , $184-185$

Harigaya Y, Saido TC, Eckman CB, Prada C-M, Shoji M, Younkin SG (2000): Amyloid $\beta$ Protein Starting Pyroglutamate at Position 3 Is a Major Component of the Amyloid Deposits in the Alzheimer's Disease Brain. Biochem Biophys Res Commun 276, 422-427

Haughey NJ, Nath A, Chan SL, Borchard AC, Rao MS, Mattson MP (2002): Disruption of neurogenesis by amyloid $\beta$-peptide, and perturbed neural progenitor cell homeostasis, in models of Alzheimer's disease. J Neurochem $\underline{83}, 1509-1524$

Haupt C, Leppert J, Rönicke R, Meinhardt J, Yadav JK, Ramachandran R, Ohlenschläger O, Reymann KG, Görlach M, Fändrich M (2012): Structural Basis of $\beta$-Amyloid-Dependent Synaptic Dysfunctions. Angew Chem Int Ed 51, 1576-1579

Hauss-Wegrzyniak B, Dobrzanski P, Stoehr JD, Wenk GL (1998): Chronic neuroinflammation in rats reproduces components of the neurobiology of Alzheimer's disease. Brain Res $\underline{780}$, 294-303

He W, Barrow CJ (1999): The A $\beta$ 3-Pyroglutamyl and 11-Pyroglutamyl Peptides Found in Senile Plaque Have Greater $\beta$-Sheet Forming and Aggregation Propensities in Vitro than FullLength $A \beta$. Biochemistry (Mosc) $\underline{38}, 10871-10877$

Heber S, Herms J, Gajic V, Hainfellner J, Aguzzi A, Rülicke T, Kretzschmar H, Koch C von, Sisodia S, Tremml P, et al. (2000): Mice with Combined Gene Knock-Outs Reveal Essential and Partially Redundant Functions of Amyloid Precursor Protein Family Members. J Neurosci $20,7951-7963$

Hebert LE, Scherr PA, McCann JJ, Bienias JL, Evans DA (2008): Change in direct measures of physical performance among persons with Alzheimer's disease. Aging Ment Health $\underline{12}$, 729-734

Heneka MT, O'Banion MK, Terwel D, Kummer MP (2010): Neuroinflammatory processes in Alzheimer's disease. J Neural Transm 117, 919-947

Herl L, Thomas AV, Lill CM, Banks M, Deng A, Jones PB, Spoelgen R, Hyman BT, Berezovska O (2009): Mutations in amyloid precursor protein affect its interactions with presenilin/gamma-secretase. Mol Cell Neurosci $\underline{41}, 166-174$

Herring A, Ambrée O, Tomm M, Habermann H, Sachser N, Paulus W, Keyvani K (2009): Environmental enrichment enhances cellular plasticity in transgenic mice with Alzheimer-like pathology. Exp Neurol 216, 184-192

Herz J, Beffert U (2000): Apolipoprotein E receptors: linking brain development and alzheimer's disease. Nat Rev Neurosci $1,51-58$ 
Higuchi M, Lee VM-Y, Trojanowski JQ (2002): Tau and axonopathy in neurodegenerative disorders. NeuroMolecular Med 2, 131-150

Hillman CH, Erickson KI, Kramer AF (2008): Be smart, exercise your heart: exercise effects on brain and cognition. Nat Rev Neurosci $\underline{9}, 58-65$

Hillmann A, Hahn S, Schilling S, Hoffmann T, Demuth H-U, Bulic B, Schneider-Axmann T, Bayer TA, Weggen S, Wirths O (2012): No improvement after chronic ibuprofen treatment in the 5XFAD mouse model of Alzheimer's disease. Neurobiol Aging 33, 833.e39$833 . e 50$

Hof PR, Bouras C, Buée L, Delacourte A, Perl DP, Morrison JH (1992): Differential distribution of neurofibrillary tangles in the cerebral cortex of dementia pugilistica and Alzheimer's disease cases. Acta Neuropathol (Berl) $\underline{85}, 23-30$

Holcomb L, Gordon MN, McGowan E, Yu X, Benkovic S, Jantzen P, Wright K, Saad I, Mueller R, Morgan D, et al. (1998): Accelerated Alzheimer-type phenotype in transgenic mice carrying both mutant amyloid precursor protein and presenilin 1 transgenes. Nat Med $\underline{4}, 97-100$

Holtzman DM, John CM, Goate A (2011): Alzheimer's Disease: The Challenge of the Second Century. Sci Transl Med $\underline{3}, 77 \mathrm{sr} 1$

Hook V, Toneff T, Bogyo M, Greenbaum D, Medzihradszky KF, Neveu J, Lane W, Hook G, Reisine T (2005): Inhibition of cathepsin B reduces $\beta$-amyloid production in regulated secretory vesicles of neuronal chromaffin cells: evidence for cathepsin B as a candidate $\beta$ secretase of Alzheimer's disease. Biol Chem 386, 931-940

Hook VYH, Kindy M, Reinheckel T, Peters C, Hook G (2009): Genetic cathepsin B deficiency reduces $\beta$-amyloid in transgenic mice expressing human wild-type amyloid precursor protein. Biochem Biophys Res Commun $\underline{386}$, 284-288

Hoozemans JJM, Veerhuis R, Rozemuller JM, Eikelenboom P (2006): Neuroinflammation and regeneration in the early stages of Alzheimer's disease pathology. Int J Dev Neurosci Off J Int Soc Dev Neurosci 24, 157-165

Hou L, Kang I, Marchant RE, Zagorski MG (2002): Methionine 35 Oxidation Reduces Fibril Assembly of the Amyloid A $\beta-(1-42)$ Peptide of Alzheimer's Disease. J Biol Chem 277, 40173-40176

Howell S, Nalbantoglu J, Crine P (1995): Neutral endopeptidase can hydrolyze $\beta$-amyloid(1-40) but shows no effect on $\beta$-amyloid precursor protein metabolism. Peptides $\underline{16}, 647-652$

Hsiao K, Chapman P, Nilsen S, Eckman C, Harigaya Y, Younkin S, Yang F, Cole G (1996): Correlative memory deficits, Abeta elevation, and amyloid plaques in transgenic mice. Science $\underline{274}, 99-102$

Hu M, Sun Y-J, Zhou Q-G, Auberson YP, Chen L, Hu Y, Luo C-X, Wu J-Y, Zhu D-Y, Li L-X (2009): Reduced spatial learning in mice treated with NVP-AAM077 through downregulating neurogenesis. Eur J Pharmacol 622, 37-44

Hussain I, Powell D, Howlett DR, Tew DG, Meek TD, Chapman C, Gloger IS, Murphy KE, Southan CD, Ryan DM, et al. (1999): Identification of a Novel Aspartic Protease (Asp 2) as $\beta$-Secretase. Mol Cell Neurosci $\underline{14}, 419-427$

Hüttenrauch M, Brauß A, Kurdakova A, Borgers H, Klinker F, Liebetanz D, Salinas-Riester G, Wiltfang J, Klafki H, Wirths O (2016): Physical activity delays hippocampal neurodegen- 
eration and rescues memory deficits in an Alzheimer disease mouse model. Transl Psychiatry 6, e800 doi: 10.1038/tp.2016.65

Hutton M, Lendon CL, Rizzu P, Baker M, Froelich S, Houlden H, Pickering-Brown S, Chakraverty S, Isaacs A, Grover A, et al. (1998): Association of missense and 5'-splice-site mutations in tau with the inherited dementia FTDP-17. Nature 393, 702-705

Hyman BT, Phelps CH, Beach TG, Bigio EH, Cairns NJ, Carrillo MC, Dickson DW, Duyckaerts C, Frosch MP, Masliah E, et al. (2012): National Institute on Aging-Alzheimer's Association guidelines for the neuropathologic assessment of Alzheimer's disease. Alzheimers Dement J Alzheimers Assoc $\underline{8}, 1-13$

Ingelsson M, Fukumoto H, Newell KL, Growdon JH, Hedley-Whyte ET, Frosch MP, Albert MS, Hyman BT, Irizarry MC (2004): Early Abeta accumulation and progressive synaptic loss, gliosis, and tangle formation in AD brain. Neurology $\underline{62}, 925-931$

Iqbal K, Grundke-Iqbal I (2008): Alzheimer neurofibrillary degeneration: significance, etiopathogenesis, therapeutics and prevention. J Cell Mol Med 12, 38-55

Iqbal K, del C. Alonso A, Chen S, Chohan MO, El-Akkad E, Gong C-X, Khatoon S, Li B, Liu F, Rahman A, et al. (2005): Tau pathology in Alzheimer disease and other tauopathies. Biochim Biophys Acta BBA - Mol Basis Dis 1739, 198-210

Irizarry MC, Soriano F, McNamara M, Page KJ, Schenk D, Games D, Hyman BT (1997): Abeta deposition is associated with neuropil changes, but not with overt neuronal loss in the human amyloid precursor protein V717F (PDAPP) transgenic mouse. J Neurosci Off J Soc Neurosci 17, 7053-7059

Itagaki S, McGeer PL, Akiyama H, Zhu S, Selkoe D (1989): Relationship of microglia and astrocytes to amyloid deposits of Alzheimer disease. J Neuroimmunol 24, 173-182

Iwata N, Tsubuki S, Takaki Y, Shirotani K, Lu B, Gerard NP, Gerard C, Hama E, Lee H-J, Saido TC (2001): Metabolic Regulation of Brain A $\beta$ by Neprilysin. Science 292, 1550-1552

Iwatsubo T, Odaka A, Suzuki N, Mizusawa H, Nukina N, Ihara Y (1994): Visualization of $A \beta 42(43)$ and $A \beta 40$ in senile plaques with end-specific $A \beta$ monoclonals: Evidence that an initially deposited species is $\mathrm{A} \beta 42(43)$. Neuron $\underline{13}, 45-53$

Jack CR, Shiung MM, Weigand SD, O'Brien PC, Gunter JL, Boeve BF, Knopman DS, Smith GE, Ivnik RJ, Tangalos EG, Petersen RC (2005): Brain atrophy rates predict subsequent clinical conversion in normal elderly and amnestic MCI. Neurology $\underline{65}, 1227-1231$

Jack CR, Knopman DS, Jagust WJ, Shaw LM, Aisen PS, Weiner MW, Petersen RC, Trojanowski JQ (2010): Hypothetical model of dynamic biomarkers of the Alzheimer's pathological cascade. Lancet Neurol $9,119-128$

Jack CR, Albert M, Knopman DS, McKhann GM, Sperling RA, Carillo M, Thies W, Phelps CH (2011): Introduction to Revised Criteria for the Diagnosis of Alzheimer's Disease: National Institute on Aging and the Alzheimer Association Workgroups. Alzheimers Dement J Alzheimers Assoc $\underline{7}, 257-262$

Jagust W (2006): Positron emission tomography and magnetic resonance imaging in the diagnosis and prediction of dementia. Alzheimers Dement J Alzheimers Assoc 2, $36-42$ 
Jan A, Gokce O, Luthi-Carter R, Lashuel HA (2008): The Ratio of Monomeric to Aggregated Forms of $A \beta 40$ and $A \beta 42$ Is an Important Determinant of Amyloid- $\beta$ Aggregation, Fibrillogenesis, and Toxicity. J Biol Chem $\underline{283}$, 28176-28189

Jankowsky JL, Melnikova T, Fadale DJ, Xu GM, Slunt HH, Gonzales V, Younkin LH, Younkin SG, Borchelt DR, Savonenko AV (2005): Environmental enrichment mitigates cognitive deficits in a mouse model of Alzheimer's disease. J Neurosci Off J Soc Neurosci $25,5217-$ 5224

Jankowsky JL, Younkin LH, Gonzales V, Fadale DJ, Slunt HH, Lester HA, Younkin SG, Borchelt DR (2007): Rodent A $\beta$ Modulates the Solubility and Distribution of Amyloid Deposits in Transgenic Mice. J Biol Chem 282, 22707-22720

Janus C, Pearson J, McLaurin J, Mathews PM, Jiang Y, Schmidt SD, Chishti MA, Horne P, Heslin $D$, French J, et al. (2000): A $\beta$ peptide immunization reduces behavioural impairment and plaques in a model of Alzheimer's disease. Nature $\underline{408}, 979-982$

Jarrett JT, Berger EP, Lansbury PT (1993): The carboxy terminus of the beta amyloid protein is critical for the seeding of amyloid formation: implications for the pathogenesis of Alzheimer's disease. Biochemistry (Mosc) $\underline{32}$, 4693-4697

Jawhar S, Trawicka A, Jenneckens C, Bayer TA, Wirths O (2012): Motor deficits, neuron loss, and reduced anxiety coinciding with axonal degeneration and intraneuronal $A \beta$ aggregation in the 5XFAD mouse model of Alzheimer's disease. Neurobiol Aging 33, 196.e29-196.e40

Jessberger S, Clark RE, Broadbent NJ, Clemenson GD, Consiglio A, Lie DC, Squire LR, Gage FH (2009): Dentate gyrus-specific knockdown of adult neurogenesis impairs spatial and object recognition memory in adult rats. Learn Mem $\underline{16}, 147-154$

Jiang W, Zhang Y, Xiao L, Van Cleemput J, Ji S-P, Bai G, Zhang X (2005): Cannabinoids promote embryonic and adult hippocampus neurogenesis and produce anxiolytic- and antidepressant-like effects. J Clin Invest $\underline{115}, 3104-3116$

Johnston JA, Liu WW, Todd SA, Coulson DTR, Murphy S, Irvine GB, Passmore AP (2005): Expression and activity of $\beta$-site amyloid precursor protein cleaving enzyme in Alzheimer's disease. Biochem Soc Trans $\underline{33}$, 1096-1100

Jonghe CD, Esselens C, Kumar-Singh S, Craessaerts K, Serneels S, Checler F, Annaert W, Broeckhoven CV, Strooper BD (2001): Pathogenic APP mutations near the $\gamma$-secretase cleavage site differentially affect A $\beta$ secretion and APP C-terminal fragment stability. Hum Mol Genet 10, 1665-1671

Kaether C, Haass C, Steiner H (2006): Assembly, Trafficking and Function of \&amp;gamma;Secretase. Neurodegener Dis $\underline{3}, 275-283$

Kalback W, Watson MD, Kokjohn TA, Kuo Y-M, Weiss N, Luehrs DC, Lopez J, Brune D, Sisodia SS, Staufenbiel M, et al. (2002): APP Transgenic Mice Tg2576 Accumulate A $\beta$ Peptides That Are Distinct from the Chemically Modified and Insoluble Peptides Deposited in Alzheimer's Disease Senile Plaques $\dagger$. Biochemistry (Mosc) 41, 922-928

Kandel ER, Schwartz JH, Jessel T: Principles of neural science. 4th ed.; McGraw-Hill, New York, NY 2000

Kaplan MS, Hinds JW (1977): Neurogenesis in the adult rat: electron microscopic analysis of light radioautographs. Science $197,1092-1094$ 
Kasa P, Papp H, Kovacs I, Forgon M, Penke B, Yamaguchi H (2000): Human amyloid- $\beta 1-42$ applied in vivo inhibits the fast axonal transport of proteins in the sciatic nerve of rat. Neurosci Lett $\underline{278}, 117-119$

Kawarabayashi T, Younkin LH, Saido TC, Shoji M, Ashe KH, Younkin SG (2001): AgeDependent Changes in Brain, CSF, and Plasma Amyloid $\beta$ Protein in the Tg2576 Transgenic Mouse Model of Alzheimer's Disease. J Neurosci 21, 372-381

Kayed R, Jackson GR (2009): Prefilament tau species as potential targets for immunotherapy for Alzheimer disease and related disorders. Curr Opin Immunol 21, 359-363

Kayed R, Head E, Thompson JL, McIntire TM, Milton SC, Cotman CW, Glabe CG (2003): Common Structure of Soluble Amyloid Oligomers Implies Common Mechanism of Pathogenesis. Science $\underline{300}, 486-489$

Kempermann G, Kuhn HG, Gage FH (1997): More hippocampal neurons in adult mice living in an enriched environment. Nature 386, 493-495

Kempermann G, Brandon EP, Gage FH (1998a): Environmental stimulation of 129/SvJ mice causes increased cell proliferation and neurogenesis in the adult dentate gyrus. Curr Biol $\underline{8}$, 939-944

Kempermann G, Kuhn HG, Gage FH (1998b): Experience-Induced Neurogenesis in the Senescent Dentate Gyrus. J Neurosci 18, 3206-3212

Kempermann G, Song H, Gage FH (2015): Neurogenesis in the Adult Hippocampus. Cold Spring Harb Perspect Biol 7, a018812

Kidd M (1963): Paired helical filaments in electron microscopy of Alzheimer's disease. Nature $\underline{197,192-193}$

Kienlen-Campard P, Miolet S, Tasiaux B, Octave J-N (2002): Intracellular Amyloid- $\beta 1-42$, but Not Extracellular Soluble Amyloid- $\beta$ Peptides, Induces Neuronal Apoptosis. J Biol Chem $\underline{277}, 15666-15670$

Kivipelto M, Helkala E-L, Laakso MP, Hänninen T, Hallikainen M, Alhainen K, Soininen H, Tuomilehto J, Nissinen A (2001): Midlife vascular risk factors and Alzheimer's disease in later life: longitudinal, population based study. BMJ $\underline{322}$, 1447-1451

Kivipelto M, Ngandu T, Fratiglioni L, Viitanen M, Kåreholt I, Winblad B, Helkala E-L, Tuomilehto J, Soininen H, Nissinen A (2005): Obesity and vascular risk factors at midlife and the risk of dementia and Alzheimer disease. Arch Neurol 62, 1556-1560

Knopman DS (2009): Mediterranean diet and late-life cognitive impairment: a taste of benefit. JAMA $302,686-687$

Knopman DS, Parisi JE, Salviati A, Floriach-Robert M, Boeve BF, Ivnik RJ, Smith GE, Dickson DW, Johnson KA, Petersen LE, et al. (2003): Neuropathology of cognitively normal elderly. J Neuropathol Exp Neurol 62, 1087-1095

Koenigsknecht-Talboo J, Meyer-Luehmann M, Parsadanian M, Garcia-Alloza M, Finn MB, Hyman BT, Bacskai BJ, Holtzman DM (2008): Rapid Microglial Response Around Amyloid Pathology after Systemic Anti-A $\beta$ Antibody Administration in PDAPP Mice. J Neurosci $\underline{28}, 14156-14164$ 
Kögel D, Schomburg R, Copanaki E, Prehn JHM (2005): Regulation of gene expression by the amyloid precursor protein: inhibition of the JNK/c-Jun pathway. Cell Death Differ 12, 1-9

Kokaia Z, Lindvall O (2003): Neurogenesis after ischaemic brain insults. Curr Opin Neurobiol $\underline{13}$, $127-132$

Korenberg JR, Pulst SM, Neve RL, West R (1989): The Alzheimer amyloid precursor protein maps to human chromosome 21 bands q21.105-q21.05. Genomics $\underline{5}, 124-127$

Kotilinek LA, Bacskai B, Westerman M, Kawarabayashi T, Younkin L, Hyman BT, Younkin S, Ashe KH (2002): Reversible Memory Loss in a Mouse Transgenic Model of Alzheimer's Disease. J Neurosci 22, 6331-6335

Kraepelin E: Psychiatrie: ein Lehrbuch für Studierende und Ärzte. 8th ed.; Barth, Leipzig 1910

Krause DL, Müller N (2010): Neuroinflammation, microglia and implications for antiinflammatory treatment in Alzheimer's disease. Int J Alzheimers Dis $\underline{2010}$

Kril JJ, Hodges J, Halliday G (2004): Relationship between hippocampal volume and CA1 neuron loss in brains of humans with and without Alzheimer's disease. Neurosci Lett $\underline{361}, 9-12$

Kuhn P-H, Wang H, Dislich B, Colombo A, Zeitschel U, Ellwart JW, Kremmer E, Roßner S, Lichtenthaler SF (2010): ADAM10 is the physiologically relevant, constitutive $\alpha$-secretase of the amyloid precursor protein in primary neurons. EMBO J 29, 3020-3032

Kumar S, Rezaei-Ghaleh N, Terwel D, Thal DR, Richard M, Hoch M, Mc Donald JM, Wüllner U, Glebov K, Heneka MT, et al. (2011): Extracellular phosphorylation of the amyloid $\beta$ peptide promotes formation of toxic aggregates during the pathogenesis of Alzheimer's disease. EMBO J $\underline{30}, 2255-2265$

Kummer MP, Heneka MT (2014): Truncated and modified amyloid-beta species. Alzheimers Res Ther $\underline{6}, 28$

Kuo Y-M, Emmerling MR, Woods AS, Cotter RJ, Roher AE (1997): Isolation, Chemical Characterization, and Quantitation of A $\beta$ 3-Pyroglutamyl Peptide from Neuritic Plaques and Vascular Amyloid Deposits. Biochem Biophys Res Commun 237, 188-191

LaMonte BH, Wallace KE, Holloway BA, Shelly SS, Ascaño J, Tokito M, Van Winkle T, Howland DS, Holzbaur ELF (2002): Disruption of Dynein/Dynactin Inhibits Axonal Transport in Motor Neurons Causing Late-Onset Progressive Degeneration. Neuron 34, 715-727

Lannfelt L, Relkin NR, Siemers ER (2014): Amyloid-ß-directed immunotherapy for Alzheimer's disease. J Intern Med 275, 284-295

Larson ME, Lesné SE (2012): Soluble A $\beta$ oligomer production and toxicity. J Neurochem $\underline{120}$, $125-139$

Lautenschlager NT, Cox KL, Flicker L, Foster JK, van Bockxmeer FM, Xiao J, Greenop KR, Almeida OP (2008): Effect of physical activity on cognitive function in older adults at risk for Alzheimer disease: a randomized trial. JAMA 300, 1027-1037

Lawton DM, Gasquoine PG, Weimer AA (2015): Age of dementia diagnosis in community dwelling bilingual and monolingual Hispanic Americans. Cortex $\underline{66}, 141-145$ 
Lazarov O, Robinson J, Tang Y-P, Hairston IS, Korade-Mirnics Z, Lee VM-Y, Hersh LB, Sapolsky RM, Mirnics K, Sisodia SS (2005): Environmental Enrichment Reduces A $\beta$ Levels and Amyloid Deposition in Transgenic Mice. Cell $\underline{120}, 701-713$

Lee VM, Balin BJ, Otvos L, Trojanowski JQ (1991): A68: a major subunit of paired helical filaments and derivatized forms of normal Tau. Science 251, 675-678

Lenders M-B, Peers M-C, Tramu G, Delacourte A, Defossez A, Petit H, Mazzuca M (1989): Dystrophic peptidergic neurites in senile plaques of Alzheimer's disease hippocampus precede formation of paired helical filaments. Brain Res $\underline{481}$, 344-349

Lesné S, Kotilinek L, Ashe KH (2008): Plaque-bearing mice with reduced levels of oligomeric amyloid- $\beta$ assemblies have intact memory function. Neuroscience $\underline{151}, 745-749$

Lewis H, Beher D, Cookson N, Oakley A, Piggott M, Morris CM, Jaros E, Perry R, Ince P, Kenny RA, et al. (2006): Quantification of Alzheimer pathology in ageing and dementia: agerelated accumulation of amyloid- $\beta(42)$ peptide in vascular dementia. Neuropathol Appl Neurobiol 32, 103-118

Liao M-C, Ahmed M, Smith SO, Nostrand WEV (2009): Degradation of Amyloid $\beta$ Protein by Purified Myelin Basic Protein. J Biol Chem 284, 28917-28925

Li S, Hong S, Shepardson NE, Walsh DM, Shankar GM, Selkoe D (2009): Soluble Oligomers of Amyloid $\beta$ Protein Facilitate Hippocampal Long-Term Depression by Disrupting Neuronal Glutamate Uptake. Neuron $\underline{62}, 788-801$

Liu C-C, Kanekiyo T, Xu H, Bu G (2013): Apolipoprotein E and Alzheimer disease: risk, mechanisms, and therapy. Nat Rev Neurol $\underline{9}, 106-118$

Liu R-Q, Zhou Q-H, Ji S-R, Zhou Q, Feng D, Wu Y, Sui S-F (2010): Membrane Localization of $\beta$ Amyloid 1-42 in Lysosomes. J Biol Chem 285, 19986-19996

Livak KJ, Schmittgen TD (2001): Analysis of Relative Gene Expression Data Using Real-Time Quantitative PCR and the 2- $\Delta \Delta \mathrm{CT}$ Method. Methods 25, 402-408

Lledo P-M, Alonso M, Grubb MS (2006): Adult neurogenesis and functional plasticity in neuronal circuits. Nat Rev Neurosci $\underline{7}, 179-193$

Lue L-F, Kuo Y-M, Roher AE, Brachova L, Shen Y, Sue L, Beach T, Kurth JH, Rydel RE, Rogers J (1999): Soluble Amyloid $\beta$ Peptide Concentration as a Predictor of Synaptic Change in Alzheimer's Disease. Am J Pathol 155, 853-862

Mandarim-de-Lacerda CA (2003): Stereological tools in biomedical research. An Acad Bras Ciênc $\underline{75}, 469-486$

Marlatt MW, Potter MC, Bayer TA, van Praag H, Lucassen PJ (2013): Prolonged running, not fluoxetine treatment, increases neurogenesis, but does not alter neuropathology, in the 3xTg mouse model of Alzheimer's disease. Curr Top Behav Neurosci 15, 313-340

Martinelli JE, Cecato JF, Bartholomeu D, Montiel JM (2014): Comparison of the Diagnostic Accuracy of Neuropsychological Tests in Differentiating Alzheimer's Disease from Mild Cognitive Impairment: Can the Montreal Cognitive Assessment Be Better than the Cambridge Cognitive Examination? Dement Geriatr Cogn Disord EXTRA 4, 113-121

Masliah E, Terry RD, Mallory M, Alford M, Hansen LA (1990): Diffuse plaques do not accentuate synapse loss in Alzheimer's disease. Am J Pathol 137, 1293-1297 
Masliah E, Ellisman M, Carragher B, Mallory M, Young S, Hansen L, DeTeresa R, Terry RD (1992): Three-dimensional analysis of the relationship between synaptic pathology and neuropil threads in Alzheimer disease. J Neuropathol Exp Neurol 51, 404-414

Masters CL, Simms G, Weinman NA, Multhaup G, McDonald BL, Beyreuther K (1985): Amyloid plaque core protein in Alzheimer disease and Down syndrome. Proc Natl Acad Sci U S A $\underline{82}, 4245-4249$

Masuhr KF, Neumann M: Neurologie. (Duale Reihe 2198-915X; Duale Reihe), 6. Auflage; Georg Thieme Verlag, Stuttgart 2007

Matsumoto S, Udaka F, Kameyama M, Kusaka H, Ito H, Imai T (1996): Subcortical neurofibrillary tangles, neuropil threads, and argentophilic glial inclusions in corticobasal degeneration. Clin Neuropathol $\underline{15}, 209-214$

Matsuoka Y, Picciano M, Malester B, LaFrancois J, Zehr C, Daeschner JM, Olschowka JA, Fonseca MI, O'Banion MK, Tenner AJ, et al. (2001): Inflammatory Responses to Amyloidosis in a Transgenic Mouse Model of Alzheimer's Disease. Am J Pathol 158, 1345-1354

Mattson MP (1997): Cellular actions of beta-amyloid precursor protein and its soluble and fibrillogenic derivatives. Physiol Rev $\underline{77}, 1081-1132$

Mattsson N, Blennow K, Zetterberg H (2009): CSF Biomarkers. Ann N Y Acad Sci 1180, 28-35

Maurer K, Maurer U: Alzheimer: the life of a physician and the career of a disease. Columbia University Press, New York 2003

McCullagh CD, Craig D, McIlroy SP, Passmore AP (2001): Risk factors for dementia. Adv Psychiatr Treat $\underline{7}, 24-31$

McKenzie JE, Gentleman SM, Roberts GW, Graham DI, Royston MC (1994): Increased numbers of beta APP-immunoreactive neurones in the entorhinal cortex after head injury. Neuroreport $\underline{6}, 161-164$

McKhann G, Drachman D, Folstein M, Katzman R, Price D, Stadlan EM (1984): Clinical diagnosis of Alzheimer's disease: report of the NINCDS-ADRDA Work Group under the auspices of Department of Health and Human Services Task Force on Alzheimer's Disease. Neurology 34, 939-944

McKhann GM, Knopman DS, Chertkow H, Hyman BT, Jack Jr. CR, Kawas CH, Klunk WE, Koroshetz WJ, Manly JJ, Mayeux R, et al. (2011): The diagnosis of dementia due to Alzheimer's disease: Recommendations from the National Institute on Aging-Alzheimer's Association workgroups on diagnostic guidelines for Alzheimer's disease. Alzheimers Dement $\underline{7}, 263-269$

McLean CA, Cherny RA, Fraser FW, Fuller SJ, Smith MJ, Konrad Vbeyreuther, Bush AI, Masters CL (1999): Soluble pool of A $\beta$ amyloid as a determinant of severity of neurodegeneration in Alzheimer's disease. Ann Neurol 46, 860-866

McShane R, Areosa Sastre A, Minakaran N (2006): Memantine for dementia. Cochrane Database Syst Rev CD003154

Meinert CL, McCaffrey LD, Breitner JC (2009): Alzheimer's Disease Anti-inflammatory Prevention Trial (ADAPT): Design, methods, and baseline results. Alzheimers Dement J Alzheimers Assoc 5, $93-104$ 
Meißner JN, Bouter Y, Bayer TA (2015): Neuron Loss and Behavioral Deficits in the TBA42 Mouse Model Expressing N-Truncated Pyroglutamate Amyloid- $\beta 3-42$. J Alzheimers Dis JAD $\underline{45}, 471-482$

Meyer-Luehmann M, Spires-Jones TL, Prada C, Garcia-Alloza M, de Calignon A, Rozkalne A, Koenigsknecht-Talboo J, Holtzman DM, Bacskai BJ, Hyman BT (2008): Rapid appearance and local toxicity of amyloid- $\beta$ plaques in a mouse model of Alzheimer's disease. Nature $\underline{451}, 720-724$

Miller DL, Papayannopoulos IA, Styles J, Bobin SA, Lin YY, Biemann K, Iqbal K (1993): Peptide Compositions of the Cerebrovascular and Senile Plaque Core Amyloid Deposits of Alzheimer's Disease. Arch Biochem Biophys 301, 41-52

Minoshima S, Cross D (2008): In vivo imaging of axonal transport using MRI: aging and Alzheimer's disease. Eur J Nucl Med Mol Imaging 35, 89-92

Miravalle L, Calero M, Takao M, Roher AE, Ghetti B, Vidal R (2005): Amino-Terminally Truncated A $\beta$ Peptide Species Are the Main Component of Cotton Wool Plaques $\dagger$. Biochemistry (Mosc) $\underline{44}, 10810-10821$

Mirochnic S, Wolf S, Staufenbiel M, Kempermann G (2009): Age effects on the regulation of adult hippocampal neurogenesis by physical activity and environmental enrichment in the APP23 mouse model of Alzheimer disease. Hippocampus 19, 1008-1018

Mizutani T, Amano N, Sasaki H, Morimatsu Y, Mori H, Yoshimura M, Yamanouchi H, Hayakawa K, Shimada H (1990): Senile dementia of Alzheimer type characterized by laminar neuronal loss exclusively in the hippocampus, parahippocampus and medial occipitotemporal cortex. Acta Neuropathol (Berl) $\underline{80}, 575-580$

Moechars D, Lorent K, De Strooper B, Dewachter I, Van Leuven F (1996): Expression in brain of amyloid precursor protein mutated in the alpha-secretase site causes disturbed behavior, neuronal degeneration and premature death in transgenic mice. EMBO J $15,1265-1274$

Moechars D, Dewachter I, Lorent K, Reversé D, Baekelandt V, Naidu A, Tesseur I, Spittaels K, Haute CVD, Checler F, et al. (1999): Early Phenotypic Changes in Transgenic Mice That Overexpress Different Mutants of Amyloid Precursor Protein in Brain. J Biol Chem 274, 6483-6492

Mohamed A, Posse de Chaves E (2011): A $\beta$ internalization by neurons and glia. Int J Alzheimers Dis $\underline{2011}, 127984$

Mohandas E, Rajmohan V, Raghunath B (2009): Neurobiology of Alzheimer's disease. Indian J Psychiatry $\underline{51}, 55-61$

de la Monte SM, Wands JR (2008): Alzheimer's disease is type 3 diabetes-evidence reviewed. J Diabetes Sci Technol 2, 1101-1113

Morgan D, Diamond DM, Gottschall PE, Ugen KE, Dickey C, Hardy J, Duff K, Jantzen P, DiCarlo G, Wilcock D, et al. (2000): A beta peptide vaccination prevents memory loss in an animal model of Alzheimer's disease. Nature $\underline{408}, 982-985$

Mori H, Takio K, Ogawara M, Selkoe DJ (1992): Mass spectrometry of purified amyloid beta protein in Alzheimer's disease. J Biol Chem 267, 17082-17086

Morris JC, Price JL (2001): Pathologic correlates of nondemented aging, mild cognitive impairment, and early-stage alzheimer's disease. J Mol Neurosci 17, 101-118 
Mosby's medical dictionary: Mosby's dictionary of medicine, nursing \& health professions. 8th ed.; Mosby/Elsevier, St. Louis 2009

Mouton PR: Principles and practices of unbiased stereology; an introduction for bioscientists. Johns Hopkins University Press, Baltimore 2002

Mullan M, Crawford F, Axelman K, Houlden H, Lilius L, Winblad B, Lannfelt L (1992): A pathogenic mutation for probable Alzheimer's disease in the APP gene at the N-terminus of beta-amyloid. Nat Genet $\underline{1}, 345-347$

Murayama KS, Kametani F, Tabira T, Araki W (2007): A novel monoclonal antibody specific for the amino-truncated $\beta$-amyloid A $\beta 5-40 / 42$ produced from caspase-cleaved amyloid precursor protein. J Neurosci Methods $\underline{161}, 244-249$

Murayama O, Tomita T, Nihonmatsu N, Murayama M, Sun X, Honda T, Iwatsubo T, Takashima A (1999): Enhancement of amyloid $\beta 42$ secretion by 28 different presenilin 1 mutations of familial Alzheimer's disease. Neurosci Lett 265, 61-63

Nakano N, Hatakeyama Y, Fukatsu R, Hayashi S, Fujii M, Fujimori K, Takahata N (1999): Eyehead coordination abnormalities and regional cerebral blood flow in Alzheimer's disease. Prog Neuropsychopharmacol Biol Psychiatry 23, 1053-1062

Näslund J, Schierhorn A, Hellman U, Lannfelt L, Roses AD, Tjernberg LO, Silberring J, Gandy SE, Winblad B, Greengard P (1994): Relative abundance of Alzheimer A beta amyloid peptide variants in Alzheimer disease and normal aging. Proc Natl Acad Sci U S A 91, $8378-8382$

Nelson PT, Braak H, Markesbery WR (2009): Neuropathology and Cognitive Impairment in Alzheimer Disease: A Complex but Coherent Relationship. J Neuropathol Exp Neurol $\underline{68}$, 114

Nichol K, Deeny SP, Seif J, Camaclang K, Cotman CW (2009): Exercise improves cognition and hippocampal plasticity in APOE $\varepsilon 4$ mice. Alzheimers Dement J Alzheimers Assoc $\underline{5}, 287$ 294

Nichol KE, Parachikova AI, Cotman CW (2007): Three weeks of running wheel exposure improves cognitive performance in the aged Tg2576 mouse. Behav Brain Res $\underline{184}, 124-132$

Nichol KE, Poon WW, Parachikova AI, Cribbs DH, Glabe CG, Cotman CW (2008): Exercise alters the immune profile in $\mathrm{Tg} 2576$ Alzheimer mice toward a response coincident with improved cognitive performance and decreased amyloid. J Neuroinflammation $\underline{5}, 13$

Nicoll JAR, Wilkinson D, Holmes C, Steart P, Markham H, Weller RO (2003): Neuropathology of human Alzheimer disease after immunization with amyloid- $\beta$ peptide: a case report. Nat Med $9,448-452$

Nilsson P, Saito T, Saido TC (2014): New mouse model of Alzheimer's. ACS Chem Neurosci $\underline{5}$, 499-502

Nithianantharajah J, Hannan AJ (2006): Enriched environments, experience-dependent plasticity and disorders of the nervous system. Nat Rev Neurosci $\underline{7}, 697-709$

Nitsch RM, Slack BE, Wurtman RJ, Growdon JH (1992): Release of Alzheimer amyloid precursor derivatives stimulated by activation of muscarinic acetylcholine receptors. Science 258 , 304-307 
Nixon RA (2005): Endosome function and dysfunction in Alzheimer's disease and other neurodegenerative diseases. Neurobiol Aging 26, 373-382

Oakley H, Cole SL, Logan S, Maus E, Shao P, Craft J, Guillozet-Bongaarts A, Ohno M, Disterhoft J, Van Eldik L, et al. (2006): Intraneuronal beta-amyloid aggregates, neurodegeneration, and neuron loss in transgenic mice with five familial Alzheimer's disease mutations: potential factors in amyloid plaque formation. J Neurosci Off J Soc Neurosci 26, 10129-10140

O’Brien RJ, Wong PC (2011): Amyloid Precursor Protein Processing and Alzheimer's Disease. Annu Rev Neurosci 34, 185-204

Oddo S, Caccamo A, Shepherd JD, Murphy MP, Golde TE, Kayed R, Metherate R, Mattson MP, Akbari Y, LaFerla FM (2003): Triple-Transgenic Model of Alzheimer's Disease with Plaques and Tangles: Intracellular A $\beta$ and Synaptic Dysfunction. Neuron $\underline{39}$, 409-421

Ohno M, Chang L, Tseng W, Oakley H, Citron M, Klein WL, Vassar R, Disterhoft JF (2006): Temporal memory deficits in Alzheimer's mouse models: rescue by genetic deletion of BACE1. Eur J Neurosci 23, 251-260

O’Keefe J, Nadel L: The hippocampus as a cognitive map. Clarendon Press, Oxford 1978

Orgogozo J-M, Rigaud A-S, Stöffler A, Möbius H-J, Forette F (2002): Efficacy and Safety of Memantine in Patients With Mild to Moderate Vascular Dementia A Randomized, PlaceboControlled Trial (MMM 300). Stroke 33, 1834-1839

Orgogozo J-M, Gilman S, Dartigues J-F, Laurent B, Puel M, Kirby LC, Jouanny P, Dubois B, Eisner L, Flitman S, et al. (2003): Subacute meningoencephalitis in a subset of patients with AD after Abeta42 immunization. Neurology $\underline{61}, 46-54$

Paradise M, Cooper C, Livingston G (2009): Systematic review of the effect of education on survival in Alzheimer's disease. Int Psychogeriatr 21, 25-32

Parent JM (2003): Injury-induced neurogenesis in the adult mammalian brain. Neurosci Rev J Bringing Neurobiol Neurol Psychiatry $\underline{9}, 261-272$

Parsons CG, Stöffler A, Danysz W (2007): Memantine: a NMDA receptor antagonist that improves memory by restoration of homeostasis in the glutamatergic system - too little activation is bad, too much is even worse. Neuropharmacology $\underline{53}, 699-723$

Parsons CG, Danysz W, Dekundy A, Pulte I (2013): Memantine and Cholinesterase Inhibitors: Complementary Mechanisms in the Treatment of Alzheimer's Disease. Neurotox Res $\underline{24}$, 358-369

Paxinos G, Franklin KBJ: The mouse brain in stereotaxic coordinates. 2nd ed.; Academic Press, San Diego 2001

Peel E (2014): 'The living death of Alzheimer's' versus 'Take a walk to keep dementia at bay': representations of dementia in print media and carer discourse. Sociol Health Illn $\underline{36}, 885-$ 901

Pepeu G, Giovannini MG (2009): Cholinesterase inhibitors and beyond. Curr Alzheimer Res $\underline{6}$, 8696

Perrin RJ, Fagan AM, Holtzman DM (2009): Multi-modal techniques for diagnosis and prognosis of Alzheimer's disease. Nature $\underline{461}, 916-922$ 
Persad CC, Jones JL, Ashton-Miller JA, Alexander NB, Giordani B (2008): Executive Function and Gait in Older Adults With Cognitive Impairment. J Gerontol A Biol Sci Med Sci $\underline{63}$, $1350-1355$

Peskind ER, Potkin SG, Pomara N, Ott BR, Graham SM, Olin JT, McDonald S (2006): Memantine treatment in mild to moderate Alzheimer disease: a 24-week randomized, controlled trial. Am J Geriatr Psychiatry Off J Am Assoc Geriatr Psychiatry 14, 704-715

Petersen RC (2004): Mild cognitive impairment as a diagnostic entity. J Intern Med 256, 183-194

Pettersson AF, Olsson E, Wahlund L-O (2005): Motor function in subjects with mild cognitive impairment and early Alzheimer's disease. Dement Geriatr Cogn Disord 19, 299-304

Pfeifer MB S Bondolfi L Stalder A Deller T Staufenbiel M Mathews PM Jucker M (2002): Cerebral Hemorrhage After Passive Anti-AB Immunotherapy. Science 298, 1379-1379

Pigino G, Morfini G, Atagi Y, Deshpande A, Yu C, Jungbauer L, LaDu M, Busciglio J, Brady S (2009): Disruption of fast axonal transport is a pathogenic mechanism for intraneuronal amyloid beta. Proc Natl Acad Sci $\underline{106}, 5907-5912$

Pike CJ, Overman MJ, Cotman CW (1995a): Amino-terminal Deletions Enhance Aggregation of $\beta$ Amyloid Peptides in Vitro. J Biol Chem 270, 23895-23898

Pike CJ, Cummings BJ, Cotman CW (1995b): Early association of reactive astrocytes with senile plaques in Alzheimer's disease. Exp Neurol 132, 172-179

Pimplikar SW (2009): Reassessing the Amyloid Cascade Hypothesis of Alzheimer's Disease. Int J Biochem Cell Biol 41, 1261-1268

Plassman BL, Havlik RJ, Steffens DC, Helms MJ, Newman TN, Drosdick D, Phillips C, Gau BA, Welsh-Bohmer KA, Burke JR, et al. (2000): Documented head injury in early adulthood and risk of Alzheimer's disease and other dementias. Neurology 55, 1158-1166

Polinsky RJ (1998): Clinical pharmacology of rivastigmine: a new-generation acetylcholinesterase inhibitor for the treatment of alzheimer's disease. Clin Ther 20, 634-647

Portelius E, Bogdanovic N, Gustavsson MK, Volkmann I, Brinkmalm G, Zetterberg H, Winblad B, Blennow K (2010): Mass spectrometric characterization of brain amyloid beta isoform signatures in familial and sporadic Alzheimer's disease. Acta Neuropathol (Berl) 120, 185193

Portelius E, Zetterberg H, Dean RA, Marcil A, Bourgeois P, Nutu M, Andreasson U, Siemers E, Mawuenyega KG, Sigurdson WC, et al. (2012): A $\beta 1-15 / 16$ as a marker for $\gamma$-secretase inhibition in Alzheimer's disease. J Alzheimers Dis JAD $\underline{31}$, 335-341

Postina R, Schroeder A, Dewachter I, Bohl J, Schmitt U, Kojro E, Prinzen C, Endres K, Hiemke C, Blessing M, et al. (2004): A disintegrin-metalloproteinase prevents amyloid plaque formation and hippocampal defects in an Alzheimer disease mouse model. J Clin Invest 113, $1456-1464$

Powers MM, Clark G (1955): An evaluation of cresyl echt violet acetate as a Nissl stain. Stain Technol $\underline{30}, 83-88$

van Praag H, Kempermann G, Gage FH (1999): Running increases cell proliferation and neurogenesis in the adult mouse dentate gyrus. Nat Neurosci $\underline{2}, 266-270$ 
Praprotnik D, Smith MA, Richey PL, Vinters HV, Perry G (1996): Filament heterogeneity within the dystrophic neurites of senile plaques suggests blockage of fast axonal transport in Alzheimer's disease. Acta Neuropathol (Berl) 91, 226-235

Price JL, Morris JC (1999): Tangles and plaques in nondemented aging and "preclinical" Alzheimer's disease. Ann Neurol $\underline{45}, 358-368$

Price JL, McKeel Jr. DW, Buckles VD, Roe CM, Xiong C, Grundman M, Hansen LA, Petersen RC, Parisi JE, Dickson DW, et al. (2009): Neuropathology of nondemented aging: Presumptive evidence for preclinical Alzheimer disease. Neurobiol Aging $\underline{30}$, 1026-1036

Price KA, Varghese M, Sowa A, Yuk F, Brautigam H, Ehrlich ME, Dickstein DL (2014): Altered synaptic structure in the hippocampus in a mouse model of Alzheimer's disease with soluble amyloid- $\beta$ oligomers and no plaque pathology. Mol Neurodegener $\underline{9}, 41$

Prince M, Cullen M, Mann A (1994): Risk factors for Alzheimer's disease and dementia: a casecontrol study based on the MRC elderly hypertension trial. Neurology 44, 97-104

Prince M, Bryce R, Albanese E, Wimo A, Ribeiro W, Ferri CP (2013): The global prevalence of dementia: A systematic review and metaanalysis. Alzheimers Dement J Alzheimers Assoc $\underline{9}, 63-75 . e 2$

Probst A, Tolnay M, Langui D, Goedert M, Spillantini MG (1996): Pick's disease: hyperphosphorylated tau protein segregates to the somatoaxonal compartment. Acta Neuropathol (Berl) 92, 588-596

Puzzo D, Lee L, Palmeri A, Calabrese G, Arancio O (2014): Behavioral assays with mouse models of Alzheimer's disease: Practical considerations and guidelines. Biochem Pharmacol $\underline{88}$, $450-467$

Qiu C, Winblad B, Marengoni A, Klarin I, Fastbom J, Fratiglioni L (2006): Heart failure and risk of dementia and alzheimer disease: A population-based cohort study. Arch Intern Med 166, 1003-1008

Querfurth HW, LaFerla FM (2010): Alzheimer's disease. N Engl J Med 362, 329-344

Racke MM, Boone LI, Hepburn DL, Parsadainian M, Bryan MT, Ness DK, Piroozi KS, Jordan WH, Brown DD, Hoffman WP, et al. (2005): Exacerbation of Cerebral Amyloid Angiopathy-Associated Microhemorrhage in Amyloid Precursor Protein Transgenic Mice by Immunotherapy Is Dependent on Antibody Recognition of Deposited Forms of Amyloid $\beta$. J Neurosci $\underline{25}, 629-636$

Rafii MS, Aisen PS (2009): Recent developments in Alzheimer's disease therapeutics. BMC Med $\underline{7}, 7$

Raskind MA, Peskind ER, Wessel T, Yuan W (2000): Galantamine in AD: A 6-month randomized, placebo-controlled trial with a 6-month extension. The Galantamine USA-1 Study Group. Neurology $\underline{54}, 2261-2268$

Reilly JF, Games D, Rydel RE, Freedman S, Schenk D, Young WG, Morrison JH, Bloom FE (2003): Amyloid deposition in the hippocampus and entorhinal cortex: Quantitative analysis of a transgenic mouse model. Proc Natl Acad Sci 100, 4837-4842

Rhein V, Baysang G, Rao S, Meier F, Bonert A, Müller-Spahn F, Eckert A (2009): Amyloid-beta Leads to Impaired Cellular Respiration, Energy Production and Mitochondrial Electron 
Chain Complex Activities in Human Neuroblastoma Cells. Cell Mol Neurobiol 29, 1063 1071

Richard BC, Kurdakova A, Baches S, Bayer TA, Weggen S, Wirths O (2015): Gene Dosage Dependent Aggravation of the Neurological Phenotype in the 5XFAD Mouse Model of Alzheimer's Disease. J Alzheimers Dis JAD 또, 1223-1236

Ring S, Weyer SW, Kilian SB, Waldron E, Pietrzik CU, Filippov MA, Herms J, Buchholz C, Eckman CB, Korte M, et al. (2007): The Secreted $\beta$-Amyloid Precursor Protein Ectodomain APPs $\alpha$ Is Sufficient to Rescue the Anatomical, Behavioral, and Electrophysiological Abnormalities of APP-Deficient Mice. J Neurosci 27, 7817-7826

Roberts GW, Gentleman SM, Lynch A, Murray L, Landon M, Graham DI (1994): Beta amyloid protein deposition in the brain after severe head injury: implications for the pathogenesis of Alzheimer's disease. J Neurol Neurosurg Psychiatry $\underline{57}$, 419-425

Rodríguez JJ, Jones VC, Tabuchi M, Allan SM, Knight EM, LaFerla FM, Oddo S, Verkhratsky A (2008): Impaired Adult Neurogenesis in the Dentate Gyrus of a Triple Transgenic Mouse Model of Alzheimer's Disease. PLoS ONE $\underline{3,}$ e2935

Rodríguez JJ, Terzieva S, Olabarria M, Lanza RG, Verkhratsky A (2013): Enriched environment and physical activity reverse astrogliodegeneration in the hippocampus of $\mathrm{AD}$ transgenic mice. Cell Death Dis $\underline{4}$, e678

Roe CM, Xiong C, Miller JP, Morris JC (2007): Education and Alzheimer disease without dementia: support for the cognitive reserve hypothesis. Neurology $\underline{68}, 223-228$

Rogaev EI, Sherrington R, Rogaeva EA, Levesque G, Ikeda M, Liang Y, Chi H, Lin C, Holman K, Tsuda T, et al. (1995): Familial Alzheimer's disease in kindreds with missense mutations in a gene on chromosome 1 related to the Alzheimer's disease type 3 gene. Nature $\underline{376}$, $775-778$

Rogers SL, Friedhoff LT (1996): The efficacy and safety of donepezil in patients with Alzheimer's disease: results of a US Multicentre, Randomized, Double-Blind, Placebo-Controlled Trial. The Donepezil Study Group. Dement Basel Switz 7, 293-303

Rogers SL, Doody RS, Mohs RC, Friedhoff LT (1998): Donepezil improves cognition and global function in Alzheimer disease: a 15-week, double-blind, placebo-controlled study. Donepezil Study Group. Arch Intern Med 158, 1021-1031

Rolland Y, Abellan van Kan G, Vellas B (2008): Physical Activity and Alzheimer's Disease: From Prevention to Therapeutic Perspectives. J Am Med Dir Assoc 9, 390-405

Rolland Y, Abellan van Kan G, Nourhashemi F, Andrieu S, Cantet C, Guyonnet-Gillette S, Vellas B (2009): An abnormal „one-leg balance“ test predicts cognitive decline during Alzheimer's disease. J Alzheimers Dis JAD $\underline{16}, 525-531$

Rosen GD, Harry JD (1990): Brain volume estimation from serial section measurements: a comparison of methodologies. J Neurosci Methods $\underline{35}$, 115-124

Rossor MN, Newman S, Frackowiak RSJ, Lantos P, Kennedy AM (1993): Alzheimer's Disease Families with Amyloid Precursor Protein Mutationsa. Ann N Y Acad Sci 695, 198-202

Rovio S, Kåreholt I, Helkala E-L, Viitanen M, Winblad B, Tuomilehto J, Soininen H, Nissinen A, Kivipelto M (2005): Leisure-time physical activity at midlife and the risk of dementia and Alzheimer's disease. Lancet Neurol 4, 705-711 
Roychaudhuri R, Yang M, Hoshi MM, Teplow DB (2009): Amyloid $\beta$-Protein Assembly and Alzheimer Disease. J Biol Chem 284, 4749-4753

Rubio-Perez JM, Morillas-Ruiz JM (2012): A review: inflammatory process in Alzheimer's disease, role of cytokines. ScientificWorldJournal 2012, 756357

Rumble B, Retallack R, Hilbich C, Simms G, Multhaup G, Martins R, Hockey A, Montgomery P, Beyreuther K, Masters CL (1989): Amyloid A4 Protein and Its Precursor in Down's Syndrome and Alzheimer's Disease. N Engl J Med 320, 1446-1452

Runz H, Rietdorf J, Tomic I, Bernard M de, Beyreuther K, Pepperkok R, Hartmann T (2002): Inhibition of Intracellular Cholesterol Transport Alters Presenilin Localization and Amyloid Precursor Protein Processing in Neuronal Cells. J Neurosci 22, 1679-1689

Russo C, Violani E, Salis S, Venezia V, Dolcini V, Damonte G, Benatti U, D’Arrigo C, Patrone E, Carlo P, Schettini G (2002): Pyroglutamate-modified amyloid $\beta$-peptides - A $\beta N 3(\mathrm{pE})-$ strongly affect cultured neuron and astrocyte survival. J Neurochem $\underline{82}, 1480-1489$

Saido TC, Iwatsubo T, Mann DMA, Shimada H, Ihara Y, Kawashima S (1995): Dominant and differential deposition of distinct $\beta$-amyloid peptide species, $A \beta N 3(\mathrm{pE})$, in senile plaques. Neuron $\underline{14}, 457-466$

Salehi A, Delcroix J-D, Mobley WC (2003): Traffic at the intersection of neurotrophic factor signaling and neurodegeneration. Trends Neurosci $\underline{26}, 73-80$

Salehi A, Delcroix J-D, Belichenko PV, Zhan K, Wu C, Valletta JS, Takimoto-Kimura R, Kleschevnikov AM, Sambamurti K, Chung PP, et al. (2006): Increased App Expression in a Mouse Model of Down's Syndrome Disrupts NGF Transport and Causes Cholinergic Neuron Degeneration. Neuron $\underline{51}, 29-42$

Salkovic-Petrisic M, Tribl F, Schmidt M, Hoyer S, Riederer P (2006): Alzheimer-like changes in protein kinase $\mathrm{B}$ and glycogen synthase kinase-3 in rat frontal cortex and hippocampus after damage to the insulin signalling pathway. J Neurochem 96, 1005-1015

Sandbrink R, Masters CL, Beyreuther K (1996): APP Gene Family Alternative Splicing Generates Functionally Related Isoformsa. Ann N Y Acad Sci 777, 281-287

Sarasa M, Pesini P (2009): Natural non-trasgenic animal models for research in Alzheimer's disease. Curr Alzheimer Res $\underline{6}, 171-178$

Saul A, Sprenger F, Bayer TA, Wirths O (2013): Accelerated tau pathology with synaptic and neuronal loss in a novel triple transgenic mouse model of Alzheimer's disease. Neurobiol Aging $\underline{34}, 2564-2573$

Saxe MD, Malleret G, Vronskaya S, Mendez I, Garcia AD, Sofroniew MV, Kandel ER, Hen R (2007): Paradoxical influence of hippocampal neurogenesis on working memory. Proc Natl Acad Sci 104, 4642-4646

Scarmeas N, Hadjigeorgiou GM, Papadimitriou A, Dubois B, Sarazin M, Brandt J, Albert M, Marder K, Bell K, Honig LS, et al. (2004): Motor signs during the course of Alzheimer disease. Neurology $\underline{63}, 975-982$

Scarmeas N, Albert M, Brandt J, Blacker D, Hadjigeorgiou G, Papadimitriou A, Dubois B, Sarazin M, Wegesin D, Marder K, et al. (2005): Motor signs predict poor outcomes in Alzheimer disease. Neurology $\underline{64}, 1696-1703$ 
Schaeffer EL, Novaes BA, da Silva ER, Skaf HD, Mendes-Neto ÁG (2009): Strategies to promote differentiation of newborn neurons into mature functional cells in Alzheimer brain. Prog Neuropsychopharmacol Biol Psychiatry $\underline{33}, 1087-1102$

Schaeffer EL, Figueiro M, Gattaz WF (2011): Insights into Alzheimer disease pathogenesis from studies in transgenic animal models. Clinics $\underline{66}, 45-54$

Scheff SW, Price DA (1993): Synapse loss in the temporal lobe in Alzheimer's disease. Ann Neurol 33, 190-199

Scheff SW, DeKosky ST, Price DA (1990): Quantitative assessment of cortical synaptic density in Alzheimer's disease. Neurobiol Aging 11, 29-37

Schenk D (2002): Amyloid- $\beta$ immunotherapy for Alzheimer's disease: the end of the beginning. Nat Rev Neurosci $\underline{3}, 824-828$

Schlenzig D, Manhart S, Cinar Y, Kleinschmidt M, Hause G, Willbold D, Funke SA, Schilling S, Demuth H-U (2009): Pyroglutamate Formation Influences Solubility and Amyloidogenicity of Amyloid Peptides. Biochemistry (Mosc) 뚀, 7072-7078

Schmand B, Walstra G, Lindeboom J, Teunisse S, Jonker C (2000): Early detection of Alzheimer's disease using the Cambridge Cognitive Examination (CAMCOG). Psychol Med $\underline{30}$, 619627

Schmitz C, Hof PR (2005): Design-based stereology in neuroscience. Neuroscience 130, 813-831

Schmitz C, Rutten BPF, Pielen A, Schäfer S, Wirths O, Tremp G, Czech C, Blanchard V, Multhaup G, Rezaie P, et al. (2004): Hippocampal Neuron Loss Exceeds Amyloid Plaque Load in a Transgenic Mouse Model of Alzheimer's Disease. Am J Pathol 164, 1495-1502

Schroeter ML, Stein T, Maslowski N, Neumann J (2009): Neural Correlates of Alzheimer's Disease and Mild Cognitive Impairment: A Systematic and Quantitative Meta-Analysis involving 1,351 Patients. NeuroImage 47, 1196-1206

Schroeter S, Khan K, Barbour R, Doan M, Chen M, Guido T, Gill D, Basi G, Schenk D, Seubert P, Games D (2008): Immunotherapy Reduces Vascular Amyloid- $\beta$ in PDAPP Mice. J Neurosci $28,6787-6793$

Schupf N, Sergievsky GH (2002): Genetic and host factors for dementia in Down's syndrome. Br J Psychiatry $\underline{180}, 405-410$

Selkoe DJ (1991): The molecular pathology of Alzheimer's disease. Neuron $\underline{6}, 487-498$

Selkoe DJ (2001): Alzheimer's disease: genes, proteins, and therapy. Physiol Rev $\underline{81}, 741-766$

Selkoe DJ (2002): Alzheimer's Disease Is a Synaptic Failure. Science 298, 789-791

Selkoe DJ (2011): Alzheimer’s Disease. Cold Spring Harb Perspect Biol $\underline{3}$, a004457

Selkoe DJ, Abraham CR, Podlisny MB, Duffy LK (1986): Isolation of low-molecular-weight proteins from amyloid plaque fibers in Alzheimer's disease. J Neurochem $\underline{46}, 1820-1834$

Selkoe DJ, Podlisny MB, Joachim CL, Vickers EA, Lee G, Fritz LC, Oltersdorf T (1988): Betaamyloid precursor protein of Alzheimer disease occurs as 110- to 135-kilodalton membrane-associated proteins in neural and nonneural tissues. Proc Natl Acad Sci USA $\underline{85}$, $7341-7345$ 
Seo J-S, Leem Y-H, Lee K-W, Kim S-W, Lee J-K, Han P-L (2010): Severe motor neuron degeneration in the spinal cord of the Tg2576 mouse model of Alzheimer disease. J Alzheimers Dis JAD $\underline{21}, 263-276$

Sergeant N, Bombois S, Ghestem A, Drobecq H, Kostanjevecki V, Missiaen C, Wattez A, David JP, Vanmechelen E, Sergheraert C, Delacourte A (2003): Truncated beta-amyloid peptide species in pre-clinical Alzheimer's disease as new targets for the vaccination approach. $\mathrm{J}$ Neurochem $\underline{85}, 1581-1591$

Serrano-Pozo A, Frosch MP, Masliah E, Hyman BT (2011): Neuropathological Alterations in Alzheimer Disease. Cold Spring Harb Perspect Med 1, a006189

Shankar GM, Li S, Mehta TH, Garcia-Munoz A, Shepardson NE, Smith I, Brett FM, Farrell MA, Rowan MJ, Lemere CA, et al. (2008): Amyloid- $\beta$ protein dimers isolated directly from Alzheimer's brains impair synaptic plasticity and memory. Nat Med 14, 837-842

Shea Y-F, Chu L-W, Chan AO-K, Ha J, Li Y, Song Y-Q (2016): A systematic review of familial Alzheimer's disease: Differences in presentation of clinical features among three mutated genes and potential ethnic differences. J Formos Med Assoc 115, 67-75

Sherrington R, Rogaev EI, Liang Y, Rogaeva EA, Levesque G, Ikeda M, Chi H, Lin C, Li G, Holman K, et al. (1995): Cloning of a gene bearing missense mutations in early-onset familial Alzheimer's disease. Nature $\underline{375}, 754-760$

Shie F-S, LeBoeuf RC, Jin L-W, LeBoeur RC (2003): Early intraneuronal Abeta deposition in the hippocampus of APP transgenic mice. Neuroreport 14, 123-129

Sinha S, Anderson JP, Barbour R, Basi GS, Caccavello R, Davis D, Doan M, Dovey HF, Frigon N, Hong J, et al. (1999): Purification and cloning of amyloid precursor protein $\beta$-secretase from human brain. Nature $\underline{402}, 537-540$

Sisodia SS (1992): Beta-amyloid precursor protein cleavage by a membrane-bound protease. Proc Natl Acad Sci U S A $\underline{89}, 6075-6079$

Sisodia SS, Koo EH, Beyreuther K, Unterbeck A, Price DL (1990): Evidence that beta-amyloid protein in Alzheimer's disease is not derived by normal processing. Science $\underline{248}, 492-495$

Sivanandam TM, Thakur MK (2012): Traumatic brain injury: A risk factor for Alzheimer's disease. Neurosci Biobehav Rev $\underline{36}, 1376-1381$

Slunt HH, Thinakaran G, Koch CV, Lo AC, Tanzi RE, Sisodia SS (1994): Expression of a ubiquitous, cross-reactive homologue of the mouse beta-amyloid precursor protein (APP). J Biol Chem 269, 2637-2644

Snowdon DA (1997): Aging and Alzheimer's Disease: Lessons From the Nun Study. The Gerontologist $\underline{37}, 150-156$

Snowdon DA (2003): Healthy aging and dementia: findings from the Nun Study. Ann Intern Med $\underline{139}, 450-454$

Solà C, Mengod G, Probst A, Palacios JM (1993): Differential regional and cellular distribution of $\beta$-amyloid precursor protein messenger RNAs containing and lacking the kunitz protease inhibitor domain in the brain of human, rat and mouse. Neuroscience $\underline{53}, 267-295$ 
Soto C, (1) EMC, (1) BF, Inestrosa NC (1995): The -Helical to -Strand Transition in the Aminoterminal Fragment of the Amyloid -Peptide Modulates Amyloid Formation. J Biol Chem $\underline{270}, 3063-3067$

Sperling RA, Aisen PS, Beckett LA, Bennett DA, Craft S, Fagan AM, Iwatsubo T, Jack CR, Kaye J, Montine TJ, et al. (2011): Toward defining the preclinical stages of Alzheimer's disease: Recommendations from the National Institute on Aging-Alzheimer's Association workgroups on diagnostic guidelines for Alzheimer's disease. Alzheimers Dement J Alzheimers Assoc ㅁ, 280-292

Spillantini MG, Murrell JR, Goedert M, Farlow MR, Klug A, Ghetti B (1998): Mutation in the tau gene in familial multiple system tauopathy with presenile dementia. Proc Natl Acad Sci $\underline{95}$, 7737-7741

Stangl D, Thuret S (2009): Impact of diet on adult hippocampal neurogenesis. Genes Nutr $\underline{4}, 271-$ 282

Steffener J, Stern Y (2012): Exploring the neural basis of cognitive reserve in aging. Biochim Biophys Acta BBA - Mol Basis Dis $\underline{1822}$, 467-473

Stern Y (2002): What is cognitive reserve? Theory and research application of the reserve concept. J Int Neuropsychol Soc JINS $\underline{8}, 448-460$

Stern Y (2006): Cognitive reserve and Alzheimer disease. Alzheimer Dis Assoc Disord 20, 112 117

Stern Y (2009): Cognitive reserve. Neuropsychologia 47, 2015-2028

Stokin GB, Lillo C, Falzone TL, Brusch RG, Rockenstein E, Mount SL, Raman R, Davies P, Masliah E, Williams DS, Goldstein LSB (2005): Axonopathy and Transport Deficits Early in the Pathogenesis of Alzheimer's Disease. Science $\underline{307,1282-1288}$

Stranahan AM, Mattson MP (2008): Impact of Energy Intake and Expenditure on Neuronal Plasticity. Neuromolecular Med $\underline{10}, 209-218$

Stranahan AM, Martin B, Maudsley S (2012): Anti-inflammatory effects of physical activity in relationship to improved cognitive status in humans and mouse models of Alzheimer's Disease. Curr Alzheimer Res 9, 86-92

Sturchler-Pierrat C, Abramowski D, Duke M, Wiederhold K-H, Mistl C, Rothacher S, Ledermann B, Bürki K, Frey P, Paganetti PA, et al. (1997): Two amyloid precursor protein transgenic mouse models with Alzheimer disease-like pathology. Proc Natl Acad Sci 94, 1328713292

Su JH, Cummings BJ, Cotman CW (1998): Plaque biogenesis in brain aging and Alzheimer's disease. II. Progressive transformation and developmental sequence of dystrophic neurites. Acta Neuropathol (Berl) 96, 463-471

Sunderland T, Hill JL, Mellow AM, Lawlor BA, Gundersheimer J, Newhouse PA, Grafman JH (1989): Clock drawing in Alzheimer's disease. A novel measure of dementia severity. J Am Geriatr Soc 37, 725-729

Suzuki N, Iwatsubo T, Odaka A, Ishibashi Y, Kitada C, Ihara Y (1994): High tissue content of soluble beta 1-40 is linked to cerebral amyloid angiopathy. Am J Pathol 145, 452-460 
Takahashi RH, Almeida CG, Kearney PF, Yu F, Lin MT, Milner TA, Gouras GK (2004): Oligomerization of Alzheimer's $\beta$-Amyloid within Processes and Synapses of Cultured Neurons and Brain. J Neurosci 24, 3592-3599

Takahashi RH, Capetillo-Zarate E, Lin MT, Milner TA, Gouras GK (2013): Accumulation of Intraneuronal $\beta$-Amyloid 42 Peptides Is Associated with Early Changes in MicrotubuleAssociated Protein 2 in Neurites and Synapses. PLoS ONE $\underline{8}$, e51965

Tariot PN, Farlow MR, Grossberg GT, Graham SM, McDonald S, Gergel I, Memantine Study Group (2004): Memantine treatment in patients with moderate to severe Alzheimer disease already receiving donepezil: a randomized controlled trial. JAMA 291, 317-324

Taupin P (2009): Adult neurogenesis and the pathogenesis of Alzheimer's disease. Med Sci Monit Int Med J Exp Clin Res $\underline{15}$, LE1

Terry AV, Buccafusco JJ (2003): The Cholinergic Hypothesis of Age and Alzheimer's DiseaseRelated Cognitive Deficits: Recent Challenges and Their Implications for Novel Drug Development. J Pharmacol Exp Ther 306, 821-827

Thal DR, Rüb U, Orantes M, Braak H (2002): Phases of A beta-deposition in the human brain and its relevance for the development of AD. Neurology $\underline{58}, 1791-1800$

Theuns J, Marjaux E, Vandenbulcke M, Van Laere K, Kumar-Singh S, Bormans G, Brouwers N, Van den Broeck M, Vennekens K, Corsmit E, et al. (2006): Alzheimer dementia caused by a novel mutation located in the APP C-terminal intracytosolic fragment. Hum Mutat 27 , $888-896$

Thomas P, Fenech M (2007): A review of genome mutation and Alzheimer's disease. Mutagenesis $\underline{22}, 15-33$

Tokuda T, Fukushima T, Ikeda S, Sekijima Y, Shoji S, Yanagisawa N, Tamaoka A (1997): Plasma levels of amyloid beta proteins Abeta1-40 and Abeta1-42(43) are elevated in Down's syndrome. Ann Neurol 41, 271-273

Tomidokoro Y, Lashley T, Rostagno A, Neubert TA, Bojsen-Møller M, Braendgaard H, Plant G, Holton J, Frangione B, Révész T, Ghiso J (2005): Familial Danish dementia: co-existence of Danish and Alzheimer amyloid subunits (ADan AND A $\{$ beta $\}$ ) in the absence of compact plaques. J Biol Chem $\underline{280}$, 36883-36894

Tucker HM, Kihiko M, Caldwell JN, Wright S, Kawarabayashi T, Price D, Walker D, Scheff S, McGillis JP, Rydel RE, Estus S (2000): The Plasmin System Is Induced by and Degrades Amyloid- $\beta$ Aggregates. J Neurosci 20, 3937-3946

Tuppo EE, Arias HR (2005): The role of inflammation in Alzheimer's disease. Int J Biochem Cell Biol 37, 289-305

Um HS, Kang EB, Leem YH, Cho IH, Yang CH, Chae KR, Hwang DY, Cho JY (2008): Exercise training acts as a therapeutic strategy for reduction of the pathogenic phenotypes for Alzheimer's disease in an NSE/APPsw-transgenic model. Int J Mol Med 22, 529-539

Urbanc B, Cruz L, Le R, Sanders J, Ashe KH, Duff K, Stanley HE, Irizarry MC, Hyman BT (2002): Neurotoxic effects of thioflavin S-positive amyloid deposits in transgenic mice and Alzheimer's disease. Proc Natl Acad Sci USA 99, 13990-13995 
Uylings HBM, van Eden CG, Hofman MA (1986): Morphometry of size/volume variables and comparison of their bivariate relations in the nervous system under different conditions. $\mathrm{J}$ Neurosci Methods 18, 19-37

Van Nostrand WE, Porter M (1999): Plasmin Cleavage of the Amyloid $\beta$-Protein: Alteration of Secondary Structure and Stimulation of Tissue Plasminogen Activator Activity. Biochemistry (Mosc) $\underline{38}, 11570-11576$

Van Vickle GD, Esh CL, Kokjohn TA, Patton RL, Kalback WM, Luehrs DC, Beach TG, Newel AJ, Lopera F, Ghetti B, et al. (2008): Presenilin-1 280Glu-->Ala mutation alters C-terminal APP processing yielding longer abeta peptides: implications for Alzheimer's disease. Mol Med Camb Mass 14, 184-194

Vassar R, Bennett BD, Babu-Khan S, Kahn S, Mendiaz EA, Denis P, Teplow DB, Ross S, Amarante $P$, Loeloff $R$, et al. (1999): $\beta$-Secretase Cleavage of Alzheimer's Amyloid Precursor Protein by the Transmembrane Aspartic Protease BACE. Science 286, 735-741

Verret L, Jankowsky JL, Xu GM, Borchelt DR, Rampon C (2007): Alzheimer's-Type Amyloidosis in Transgenic Mice Impairs Survival of Newborn Neurons Derived from Adult Hippocampal Neurogenesis. J Neurosci 27, 6771-6780

Vidal M, Morris R, Grosveld F, Spanopoulou E (1990): Tissue-specific control elements of the Thy-1 gene. EMBO J $\underline{9}, 833-840$

Villemagne VL, Fodero-Tavoletti MT, Pike KE, Cappai R, Masters CL, Rowe CC (2008): The ART of Loss: A $\beta$ Imaging in the Evaluation of Alzheimer's Disease and other Dementias. Mol Neurobiol $\underline{38}, 1-15$

Villemagne VL, Pike KE, Chételat G, Ellis KA, Mulligan RS, Bourgeat P, Ackermann U, Jones G, Szoeke C, Salvado O, et al. (2011): Longitudinal assessment of $A \beta$ and cognition in aging and Alzheimer disease. Ann Neurol $\underline{69}, 181-192$

Viña J, Lloret A (2010): Why women have more Alzheimer's disease than men: gender and mitochondrial toxicity of amyloid-beta peptide. J Alzheimers Dis JAD 20 Suppl 2, 527-533

Visser PJ, Scheltens P, Verhey FRJ (2005): Do MCI criteria in drug trials accurately identify subjects with predementia Alzheimer's disease? J Neurol Neurosurg Psychiatry 76, 13481354

Vlassenko AG, Mintun MA, Xiong C, Sheline YI, Goate AM, Benzinger TLS, Morris JC (2011): Amyloid-beta plaque growth in cognitively normal adults: Longitudinal [11C]Pittsburgh compound B data. Ann Neurol 70, 857-861

Wada H, Nakajoh K, Satoh-Nakagawa T, Suzuki T, Ohrui T, Arai H, Sasaki H (2001): Risk factors of aspiration pneumonia in Alzheimer's disease patients. Gerontology 47, 271-276

Waldemar G, Dubois B, Emre M, Georges J, McKeith IG, Rossor M, Scheltens P, Tariska P, Winblad B (2007): Recommendations for the diagnosis and management of Alzheimer's disease and other disorders associated with dementia: EFNS guideline. Eur J Neurol 14, e1-e26

Wallin ÅK, Wattmo C, Minthon L (2011): Galantamine treatment in Alzheimer's disease: response and long-term outcome in a routine clinical setting. Neuropsychiatr Dis Treat $\underline{7}, 565-576$

Walsh DM, Selkoe DJ (2007): A $\beta$ Oligomers - a decade of discovery. J Neurochem $\underline{101}, 1172$ 1184 
Walsh DM, Klyubin I, Fadeeva JV, Cullen WK, Anwyl R, Wolfe MS, Rowan MJ, Selkoe DJ (2002): Naturally secreted oligomers of amyloid $\beta$ protein potently inhibit hippocampal long-term potentiation in vivo. Nature $\underline{416}, 535-539$

Weingarten MD, Lockwood AH, Hwo SY, Kirschner MW (1975): A protein factor essential for microtubule assembly. Proc Natl Acad Sci USA $\underline{72}$, 1858-1862

Welander H, Frånberg J, Graff C, Sundström E, Winblad B, Tjernberg LO (2009): A $\beta 43$ is more frequent than $\mathrm{A} \beta 40$ in amyloid plaque cores from Alzheimer disease brains. J Neurochem $\underline{110}, 697-706$

Weninger SC, Yankner BA (2001): Inflammation and Alzheimer disease: The good, the bad, and the ugly. Nat Med $\underline{7}, 527-528$

West MJ (2002): Design-based stereological methods for counting neurons. Prog Brain Res $\underline{135}$, 43-51

West MJ (2012): Introduction to stereology. Cold Spring Harb Protoc 2012, pii: pdb.top070623

West MJ, Slomianka L, Gundersen HJ (1991): Unbiased stereological estimation of the total number of neurons in thesubdivisions of the rat hippocampus using the optical fractionator. Anat $\operatorname{Rec} \underline{231}, 482-497$

West MJ, Kawas CH, Stewart WF, Rudow GL, Troncoso JC (2004): Hippocampal neurons in preclinical Alzheimer's disease. Neurobiol Aging 25, 1205-1212

Wilcock DM, DiCarlo G, Henderson D, Jackson J, Clarke K, Ugen KE, Gordon MN, Morgan D (2003): Intracranially Administered Anti-A $\beta$ Antibodies Reduce $\beta$-Amyloid Deposition by Mechanisms Both Independent of and Associated with Microglial Activation. J Neurosci $\underline{23}, 3745-3751$

Wilcock DM, Rojiani A, Rosenthal A, Levkowitz G, Subbarao S, Alamed J, Wilson D, Wilson N, Freeman MJ, Gordon MN, Morgan D (2004a): Passive Amyloid Immunotherapy Clears Amyloid and Transiently Activates Microglia in a Transgenic Mouse Model of Amyloid Deposition. J Neurosci 24, 6144-6151

Wilcock DM, Rojiani A, Rosenthal A, Subbarao S, Freeman MJ, Gordon MN, Morgan D (2004b): Passive immunotherapy against A $\beta$ in aged APP-transgenic mice reverses cognitive deficits and depletes parenchymal amyloid deposits in spite of increased vascular amyloid and microhemorrhage. J Neuroinflammation $\underline{1}, 24$

Wilcock DM, Alamed J, Gottschall PE, Grimm J, Rosenthal A, Pons J, Ronan V, Symmonds K, Gordon MN, Morgan D (2006): Deglycosylated Anti-Amyloid- $\beta$ Antibodies Eliminate Cognitive Deficits and Reduce Parenchymal Amyloid with Minimal Vascular Consequences in Aged Amyloid Precursor Protein Transgenic Mice. J Neurosci 26, 5340-5346

Willett WC (2006): The Mediterranean diet: science and practice. Public Health Nutr $\underline{9}, 105-110$

Willuweit A, Velden J, Godemann R, Manook A, Jetzek F, Tintrup H, Kauselmann G, Zevnik B, Henriksen G, Drzezga A, et al. (2009): Early-Onset and Robust Amyloid Pathology in a New Homozygous Mouse Model of Alzheimer's Disease. PLOS ONE 4, e7931

Wiltfang J, Esselmann H, Cupers P, Neumann M, Kretzschmar H, Beyermann M, Schleuder D, Jahn H, Rüther E, Kornhuber J, et al. (2001): Elevation of $\beta$-Amyloid Peptide 2-42 in Sporadic and Familial Alzheimer's Disease and Its Generation in PS1 Knockout Cells. J Biol Chem 276, 42645-42657 
Wiltfang J, Esselmann H, Bibl M, Smirnov A, Otto M, Paul S, Schmidt B, Klafki H-W, Maler M, Dyrks T, et al. (2002): Highly conserved and disease-specific patterns of carboxyterminally truncated $A \beta$ peptides 1-37/38/39 in addition to 1-40/42 in Alzheimer's disease and in patients with chronic neuroinflammation. J Neurochem $\underline{81}$, 481-496

Wines-Samuelson M, Shen J (2005): Presenilins in the Developing, Adult, and Aging Cerebral Cortex. The Neuroscientist $11,441-451$

Wirths O, Bayer TA (2008): Motor impairment in Alzheimer's disease and transgenic Alzheimer's disease mouse models. Genes Brain Behav 7 Suppl 1, 1-5

Wirths O, Bayer TA (2010): Neuron loss in transgenic mouse models of Alzheimer's disease. Int J Alzheimers Dis 2010, e723782

Wirths O, Bayer TA (2012): Intraneuronal A $\beta$ accumulation and neurodegeneration: Lessons from transgenic models. Life Sci $91,1148-1152$

Wirths O, Multhaup G, Czech C, Blanchard V, Moussaoui S, Tremp G, Pradier L, Beyreuther K, Bayer TA (2001): Intraneuronal A $\beta$ accumulation precedes plaque formation in $\beta$-amyloid precursor protein and presenilin-1 double-transgenic mice. Neurosci Lett $\underline{306}, 116-120$

Wirths O, Multhaup G, Bayer TA (2004): A modified $\beta$-amyloid hypothesis: intraneuronal accumulation of the $\beta$-amyloid peptide - the first step of a fatal cascade. J Neurochem $91,513-$ 520

Wirths O, Weis J, Szczygielski J, Multhaup G, Bayer TA (2006): Axonopathy in an APP/PS1 transgenic mouse model of Alzheimer's disease. Acta Neuropathol (Berl) 111, 312-319

Wirths O, Weis J, Kayed R, Saido TC, Bayer TA (2007): Age-dependent axonal degeneration in an Alzheimer mouse model. Neurobiol Aging 28, 1689-1699

Wirths O, Breyhan H, Cynis H, Schilling S, Demuth H-U, Bayer TA (2009): Intraneuronal pyroglutamate-Abeta 3-42 triggers neurodegeneration and lethal neurological deficits in a transgenic mouse model. Acta Neuropathol (Berl) $\underline{118}$, 487-496

Wirths O, Erck C, Martens H, Harmeier A, Geumann C, Jawhar S, Kumar S, Multhaup G, Walter $\mathrm{J}$, Ingelsson M, et al. (2010a): Identification of Low Molecular Weight Pyroglutamate A $\beta$ Oligomers in Alzheimer Disease: a novel tool for therapie and diagnosis. J Biol Chem $\underline{285}$, 41517-41524

Wirths O, Breyhan H, Marcello A, Cotel M-C, Brück W, Bayer TA (2010b): Inflammatory changes are tightly associated with neurodegeneration in the brain and spinal cord of the APP/PS1KI mouse model of Alzheimer's disease. Neurobiol Aging $\underline{31}$, 747-757

Wirths O, Bethge T, Marcello A, Harmeier A, Jawhar S, Lucassen PJ, Multhaup G, Brody DL, Esparza T, Ingelsson M, et al. (2010c): Pyroglutamate Abeta pathology in APP/PS1KI mice, sporadic and familial Alzheimer's disease cases. J Neural Transm 117, 85-96

Wisniewski T (2009): AD Vaccines: Conclusions and Future Directions. CNS Neurol Disord Drug Targets $\underline{8}, 160-166$

Wisniewski T, Konietzko U (2008): Amyloid- $\beta$ immunisation for Alzheimer's disease. Lancet Neurol $\underline{7}, 805-811$

Witman GB, Cleveland DW, Weingarten MD, Kirschner MW (1976): Tubulin requires tau for growth onto microtubule initiating sites. Proc Natl Acad Sci USA $\underline{73}, 4070-4074$ 
Wittnam JL, Portelius E, Zetterberg H, Gustavsson MK, Schilling S, Koch B, Demuth H-U, Blennow K, Wirths O, Bayer TA (2012): Pyroglutamate Amyloid $\beta$ (A $\beta$ ) Aggravates Behavioral Deficits in Transgenic Amyloid Mouse Model for Alzheimer Disease. J Biol Chem 287, $8154-8162$

Wolf SA, Kronenberg G, Lehmann K, Blankenship A, Overall R, Staufenbiel M, Kempermann G (2006): Cognitive and Physical Activity Differently Modulate Disease Progression in the Amyloid Precursor Protein (APP)-23 Model of Alzheimer's Disease. Biol Psychiatry $\underline{60}$, $1314-1323$

Wolfe MS (2007): When loss is gain: reduced presenilin proteolytic function leads to increased A $342 / A \beta 40$. Talking Point on the role of presenilin mutations in Alzheimer disease. EMBO Rep $\underline{8}, 136-140$

Woodruff-Pak DS (2008): Animal models of Alzheimer's disease: therapeutic implications. J Alzheimers Dis JAD $\underline{15}, 507-521$

Xiao A-W, He J, Wang Q, Luo Y, Sun Y, Zhou Y-P, Guan Y, Lucassen PJ, Dai J-P (2011): The origin and development of plaques and phosphorylated tau are associated with axonopathy in Alzheimer's disease. Neurosci Bull 27, 287-299

Yagita Y, Kitagawa K, Sasaki T, Miyata T, Okano H, Hori M, Matsumoto M (2002): Differential expression of Musashi1 and nestin in the adult rat hippocampus after ischemia. J Neurosci Res $\underline{69}, 750-756$

Yamaguchi H, Hirai S, Morimatsu M, Shoji M, Harigaya Y (1988): Diffuse type of senile plaques in the brains of Alzheimer-type dementia. Acta Neuropathol (Berl) 77, 113-119

Yan JH, Rountree S, Massman P, Doody RS, Li H (2008): Alzheimer's disease and mild cognitive impairment deteriorate fine movement control. J Psychiatr Res 42, 1203-1212

Yan R, Bienkowski MJ, Shuck ME, Miao H, Tory MC, Pauley AM, Brashler JR, Stratman NC, Mathews WR, Buhl AE, et al. (1999): Membrane-anchored aspartyl protease with Alzheimer's disease $\beta$-secretase activity. Nature $\underline{402}, 533-537$

Yang L-B, Lindholm K, Yan R, Citron M, Xia W, Yang X-L, Beach T, Sue L, Wong P, Price D, et al. (2003): Elevated $\beta$-secretase expression and enzymatic activity detected in sporadic Alzheimer disease. Nat Med $\underline{9}, 3-4$

Young-Pearse TL, Bai J, Chang R, Zheng JB, LoTurco JJ, Selkoe DJ (2007): A Critical Function for $\beta$-Amyloid Precursor Protein in Neuronal Migration Revealed by In Utero RNA Interference. J Neurosci 27, 14459-14469

Yuan Q, Su H, Zhang Y, Chau WH, Ng CT, Song Y-Q, Huang J-D, Wu W, Lin Z-X (2013): Amyloid pathology in spinal cord of the transgenic Alzheimer's disease mice is correlated to the corticospinal tract pathway. J Alzheimers Dis JAD $\underline{35}, 675-685$

Yuede CM, Dong H, Csernansky JG (2007): Anti-dementia drugs and hippocampal-dependent memory in rodents. Behav Pharmacol 18, 347-363

Zetterberg H, Mattsson N (2014): Understanding the cause of sporadic Alzheimer's disease. Expert Rev Neurother 14, 621-630

Zhang C, Wu B, Beglopoulos V, Wines-Samuelson M, Zhang D, Dragatsis I, Südhof TC, Shen J (2009a): Presenilins are essential for regulating neurotransmitter release. Nature $\underline{460}, 632$ 636 
Zhang R, Zhang Z, Wang L, Wang Y, Gousev A, Zhang L, Ho K-L, Morshead C, Chopp M (2004): Activated Neural Stem Cells Contribute to Stroke-Induced Neurogenesis and Neuroblast Migration Toward the Infarct Boundary in Adult Rats. J Cereb Blood Flow Metab $\underline{24}, 441-448$

Zhang X-M, Cai Y, Xiong K, Cai H, Luo X-G, Feng J-C, Clough RW, Struble RG, Patrylo PR, Yan X-X (2009b): $\beta$-Secretase-1 elevation in transgenic mouse models of Alzheimer's disease is associated with synaptic/axonal pathology and amyloidogenesis: implications for neuritic plaque development. Eur J Neurosci $\underline{30}$, 2271-2283

Zhang Y, Thompson R, Zhang H, Xu H (2011): APP processing in Alzheimer's disease. Mol Brain $\underline{4}, 3$

Zheng H, Koo EH (2006): The amyloid precursor protein: beyond amyloid. Mol Neurodegener 1,5

Zheng H, Jiang M, Trumbauer ME, Sirinathsinghji DJ, Hopkins R, Smith DW, Heavens RP, Dawson GR, Boyce S, Conner MW, et al. (1995): beta-Amyloid precursor protein-deficient mice show reactive gliosis and decreased locomotor activity. Cell $\underline{81}, 525-531$

Zhou S, Zhou H, Walian PJ, Jap BK (2006): The discovery and role of CD147 as a subunit of gamma-secretase complex. Drug News Perspect 19, 133-138

Ziabreva I, Perry E, Perry R, Minger SL, Ekonomou A, Przyborski S, Ballard C (2006): Altered neurogenesis in Alzheimer's disease. J Psychosom Res 61, 311-316

Zitnik G, Martin GM (2002): Age-related decline in neurogenesis: old cells or old environment? J Neurosci Res $\underline{70}, 258-263$ 


\section{Online source material}

Deutsche Alzheimer Gesellschaft (2014): www.deutsche-alzheimer.de. "Das Wichtigste über die Alzheimer-Krankheit. Infoblatt zur Verbreitung der Demenzen. Juni 2014". Heruntergeladen am 04. Oktober, 2015 von https://www.deutsche-alzheimer.de/die-krankheit/diealzheimer-krankheit.html. PDF-link: https://www.deutsche-alzheimer.de/fileadmin/alz/ pdf/factsheets/infoblatt1_haeufigkeit_demenzerkrankungen_dalzg.pdf

NIH, National Institute of Aging (2011): www.nia.nih.gov. "Global Health and Aging. NIH Publication No. 11-7737. Oktober 2011". Heruntergeladen am 04. Oktober, 2015 von https://www.nia.nih.gov/research/publication/global-health-and-aging/living-longer. PDFlink: https://d2cauhfh6h4x0p.cloudfront.net/s3fs-public/global_health_and_aging.pdf

NIH, National Institute of Aging (2015): www.nia.nih.gov. "Alzheimer's Disease Fact Sheet. NIH Publication No. 15-6423. May 2015“. Heruntergeladen am 04. Oktober, 2015 von https://www.nia.nih.gov/alzheimers/publication/alzheimers-disease-fact-sheet. PDF-link: https://d2cauhfh6h4x0p.cloudfront.net/s3fs-public/ad_fact_sheet-2015_update-final.pdf

World Alzheimer Report (2015): „World Alzheimer Report 2015: The Global Impact of Dementia. August 2015”. Alzheimer's Disease International. London 2015. Heruntergeladen am 04. Oktober, 2015 von http://www.alz.co.uk/research/world-report-2015. PDF-link: https://www.alz.co.uk/research/WorldAlzheimerReport2015.pdf

World Health Organization (2015): www.who.int. „Dementia. Fact sheet on dementia no. 362. March 2015“. Abfrage am 01. September, 2015 von http://www.who.int/mediacentre/factsheets/fs362/en/

www.corning.com. Figure "15mm Netwell ${ }^{\mathrm{TM}}$ Insert with $440 \mu \mathrm{m}$ Mesh Size Polyester Membrane, Sterile". Heruntergeladen am 26. August, 2015 von http://catalog2.corning.com/LifeSciences/enUS/Shopping/ProductDetails.aspx $?$ categoryname $=\&$ productid $=3478 \% 28$ Lifesciences $\% 29$

www.memory.ucsf.edu/education/diseases/alzheimer. Figure. Heruntergeladen am 02. Mai 2016

www.mbfbioscience.com. "Stereo Investigator User Guide. Guard zones". Heruntergeladen am 26. August, 2015 von

http://www.mbfbioscience.com/help/si11/Content/SI_SPECIFIC/Practical\%20concepts/gu ard_zones.htm

www.neuropathology-web.org. "Chapter 9. Degenerative diseases. Alzheimer's disease. Pathology of AD. Diffuse plaque. Neurofibrillary tangle and neuritic plaque. Neurofibrillary tangles". Heruntergeladen am 12. September, 2015 von http://neuropathologyweb.org/chapter9/chapter9bAD.html

www.nyamcenterforhistory.org. „The Legacy of Aloysius “Alois” Alzheimer”. Heruntergeladen am 04. Oktober, 2015 http://nyamcenterforhistory.org/2015/06/15/the-legacy-of-aloysiusalois-alzheimer/

www.stereology.info. "Counting Rules". Heruntergeladen am 26. August, 2015 von http://www.stereology.info/counting-rules/

www.stereology.info. "Criteria for Counting Cells". Heruntergeladen am 26. August, 2015 von http://www.stereology.info/criteria-for-counting-cells/

www.stereology.info. "The Optical Fractionator". Heruntergeladen am 26. August, 2015 von http://www.stereology.info/the-optical-fractionator/ 


\section{ACKNOWLEDGEMENTS}

First of all, I would like to express my gratitude to my supervisor PD Dr. Oliver Wirths for making it possible for me to carry out this thesis. Thank you very much for being available during the whole time of my work on this thesis to explain me things, discuss results, make plans, and support me. You are the best supervisor that I could ever wish for.

The present thesis would not have been possible without Prof. Dr. Thomas Bayer. I very much appreciate your work in the field of Alzheimer's disease and leading the team of Molecular Psychiatry. Thanks a lot for your help.

I am very grateful to my colleague Melanie Hüttenrauch for teaching me and helping me. Thank you for filling me with enthusiasm and for your understanding. Many thanks go to Bernhard Richard for his help and teaching, and to Yvonne Bouter for teaching me the stereology. Thanks to my colleagues, Andreas Brauß, Henning Borgers, Jochim Rainert, and Julius Meißner for help and discussions. Many thanks for Petra Tucholla for her expert technical assistance. A deep gratitude to the whole team of Prof. Bayer's lab.

I would like to thank the Georg-August-University of Göttingen for giving me the opportunity to participate in Jacob-Henle-Program for Experimental Medicine. A lot of appreciation goes to PD Dr. Werner Albig for organising the Program and supervising it.

I am deeply grateful and proud to be the recipient of the Friedrich Ebert Foundation fellowship. With its financial assistance, Friedrich Ebert Foundation enabled me in difficult times to pursue my medical studies and scientific research in Germany. 